Supporting information for

\title{
3'-Sulfo-TF Antigen Determined by GAL3ST2/ST3GAL1 is Essential for Antitumour Activity of Fungal Galectin AAL/AAGL
}

\author{
Yang $\mathrm{Li}^{1}$, Yan $\mathrm{Li}^{1}$, Jing Xia ${ }^{1}$, Qing Yang ${ }^{2}$, Yijie Chen ${ }^{3 *}$, and Hui Sun ${ }^{1,4^{*}}$
}

${ }^{1}$ College of Life Sciences, Wuhan University, Wuhan, 430071, Hubei Province, P. R.

China

${ }^{2}$ College of Food Science and Engineering, Wuhan Polytechnic University, Wuhan, 430023, Hubei Province, P. R. China

${ }^{3}$ College of Food Science and Technology, Huazhong Agricultural University, Wuhan, 430070, Hubei Province, P. R. China

${ }^{4}$ Hubei Province key Laboratory of Allergy and Immunology; Wuhan University, Wuhan, 430071, Hubei Province, P. R. China

*Corresponding authors:

Email: sunhui@whu.edu.cn(H. Sun); yijiechen@mail.hzau.edu.cn (Y.J. Chen) 
A

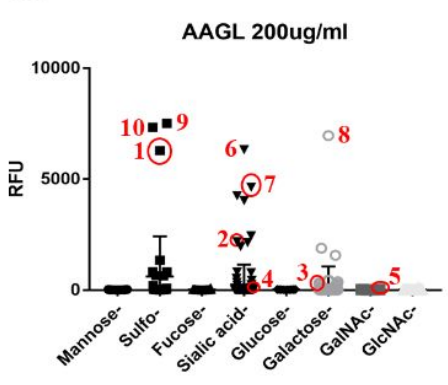

$\mathrm{D}$
B

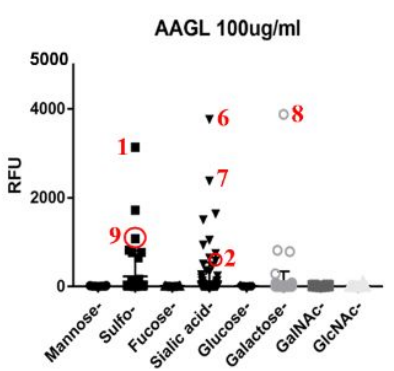

$\mathrm{C}$

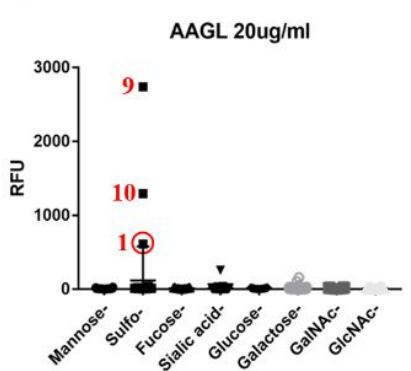

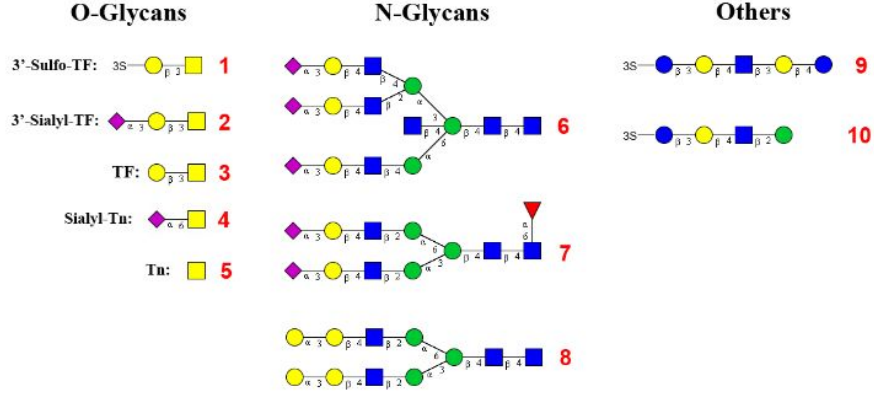

Figure S1. The glycan array analysis of AAL/AAGL according to the terminal-glycans at different concentrations (A) $200 \mu \mathrm{g} / \mathrm{ml}$; (B) $100 \mu \mathrm{g} / \mathrm{ml}$; (C) $20 \mu \mathrm{g} / \mathrm{ml}$; (D) Those main glycotypes indicated in $\mathrm{A}, \mathrm{B}$ and $\mathrm{C}$ were show high affintiy with AAL/AAGL. Glycotype 1-5 are TF-related O-glycans. Glycotype 6-8 are N-glycans which highly expressed on the surface of tumor cells. However, glycotype 8 and 9 are uncommon in mammals. 
A
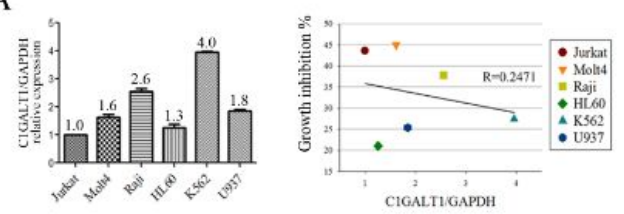

C

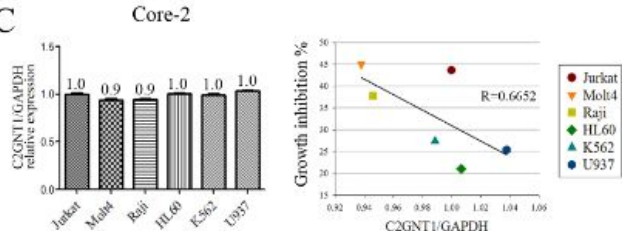

E
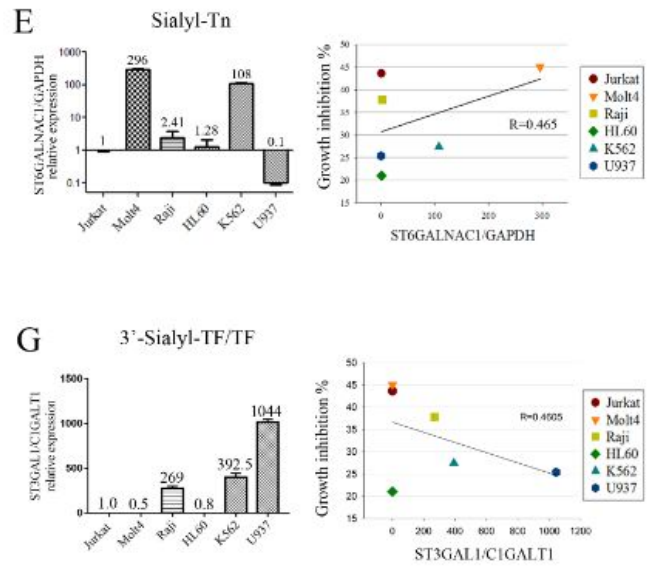

B
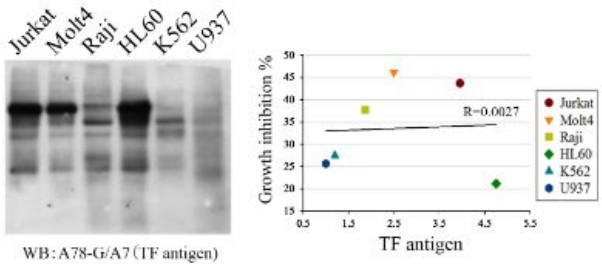

D

Core-2
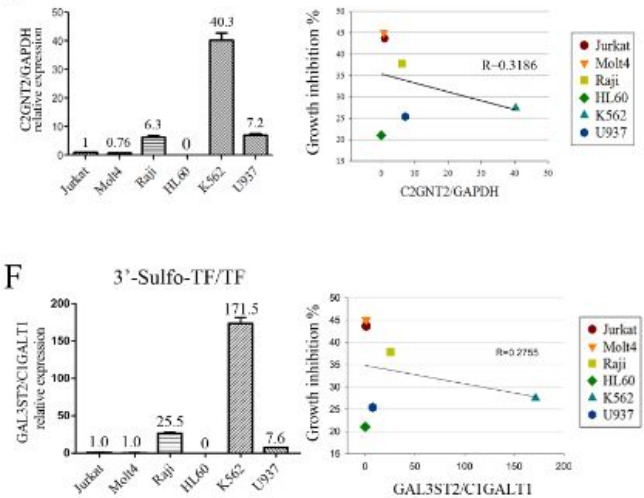

Figure S2. The TF-related antigen synthesis glycosyltransferases and TF antigen expression level are not correlated with the tumor inhibition activity of AAL/AAGL. (A,B) TF synthesis enzyme and TF expression level; (C,D) Core-2 synthesis enzymes; (E) 3'-sialyl-Tn synthesis enzyme; (F) synthesis enzymes ratio of 3'-sulfo-TF/TF; (G) synthesis enzymes ratio of 3'-sialyl-TF/TF are analysed the correlation with the tumor growth inhibition rate of AAL/AAGL.

Supporting data 1. AAL-200ug_14734_V5.0_DATA

\begin{tabular}{|c|c|c|c|c|}
\hline $\begin{array}{l}\text { Chart } \\
\text { Number }\end{array}$ & AAL (200ug/ml) CFG\#2473 Slide\#:14734 11/29/2011 Alexa488 CMZ & Average RFU & StDev & $\% \mathrm{CV}$ \\
\hline 364 & Gala1-3Galb1-4GIcNAcb1-2Mana1-6(Gala1-3Galb1-4GlcNAcb1-2Mana1-3)Manb1-4GlcNAcb1-4GlcNAcb-Sp20 & 10176 & 379 & 4 \\
\hline 571 & (3S)GIcAb1-3Galb1-4GIcNAcb1-2Mana-Sp0 & 7512 & 541 & 7 \\
\hline 570 & (3S)GlcAb1-3Galb1-4GlcNAcb1-3Galb1-4Glc-Sp0 & 7335 & 272 & 4 \\
\hline 561 & Gala1-3Galb1-4GIcNAcb1-2Mana1-6(Gala1-3Galb1-4GIcNAcb1-2Mana1-3)Manb1-4GIcNAcb1-4GlcNAc-Sp24 & 6966 & 144 & 2 \\
\hline 461 & $\begin{array}{l}\text { Neu5Aca2-3Galb1-4GIcNAcb1-4Mana1-6(GlcNAcb1-4)(Neu5Aca2-3Galb1-4GlcNAcb1-4(Neu5Aca2-3Galb1- } \\
\text { 4GlcNAcb1-2)Mana1-3)Manb1-4GIcNAcb1-4GIcNAcb-Sp21 }\end{array}$ & 6325 & 102 & 2 \\
\hline 29 & (3S)Galb1-3GalNAca-Sp8 & 6286 & 464 & 7 \\
\hline
\end{tabular}




\begin{tabular}{|c|c|c|c|c|}
\hline 484 & $\begin{array}{l}\text { Neu5Aca2-3Galb1-4GIcNAcb1-2Mana1-6(Neu5Aca2-3Galb1-4GIcNAcb1-2Mana1-3)Manb1-4GlcNAcb1-4(Fuca1- } \\
\text { 6)GlcNAcb-Sp24 }\end{array}$ & 4636 & 104 & 2 \\
\hline 463 & $\begin{array}{l}\text { Neu5Aca2-3Galb1-4GlcNAcb1-6(Neu5Aca2-3Galb1-4GIcNAcb1-2)Mana1-6(GlcNAcb1-4)(Neu5Aca2-3Galb1- } \\
\text { 4GIcNAcb1-4(Neu5Aca2-3Galb1-4GIcNAcb1-2)Mana1-3)Manb1-4GlcNAcb1-4GlcNAcb-Sp21 }\end{array}$ & 4254 & 169 & 4 \\
\hline 326 & $\begin{array}{l}\text { Neu5Aca2-3Galb1-4GIcNAcb1-2Mana1-6(Neu5Aca2-3Galb1-4GIcNAcb1-2Mana1-3)Manb1-4GIcNAcb1- } \\
\text { 4GlcNAcb-Sp12 }\end{array}$ & 4039 & 190 & 5 \\
\hline 556 & Neu5Gca2-8Neu5Gca2-3Galb1-4GIcNAcb1-3Galb1-4GIcNAc-Sp0 & 3219 & 153 & 5 \\
\hline 460 & $\begin{array}{l}\text { Neu5Aca2-3Galb1-4GIcNAcb1-2Mana1-6(GlcNAcb1-4)(Neu5Aca2-3Galb1-4GlcNAcb1-2Mana1-3)Manb1- } \\
\text { 4GIcNAcb1-4GIcNAcb-Sp21 }\end{array}$ & 2444 & 111 & 5 \\
\hline 224 & Neu5Aca2-3Galb1-3GalNAca-Sp8 & 2178 & 331 & 15 \\
\hline 247 & Neu5Aca2-3Galb1-3GalNAcb1-3Gala1-4Galb1-4Glcb-Sp0 & 2142 & 63 & 3 \\
\hline 462 & $\begin{array}{l}\text { Neu5Aca2-3Galb1-4GlcNAcb1-6(Neu5Aca2-3Galb1-4GlcNAcb1-2)Mana1-6(GlcNAcb1-4)(Neu5Aca2-3Galb1- } \\
\text { 4GlcNAcb1-2Mana1-3)Manb1-4GlcNAcb1-4GlcNAcb-Sp21 }\end{array}$ & 1961 & 235 & 12 \\
\hline 589 & $\begin{array}{l}\text { Galb1-4GlcNAcb1-3Galb1-4GlcNAcb1-3Galb1-4GlcNAcb1-3Galb1-4GlcNAcb1-3Galb1-4GIcNAcb1-6(Galb1- } \\
\text { 4GIcNAcb1-3Galb1-4GlcNAcb1-3Galb1-4GlcNAcb1-3Galb1-4GlcNAcb1-3Galb1-4GlcNAb1-2)Mana1-6(Galb1- } \\
\text { 4GlcNAcb1-3Galb1-4GlcNAcb1-3Galb1-4GlcNAcb1-3Galb1-4GIcNAcb1-3Galb1-4GlcNAcb1-2Mana1-3)Manb1- } \\
\text { 4GlcNAcb1-4(Fuca1-6)GlcNAcb-Sp24 }\end{array}$ & 1884 & 352 & 19 \\
\hline 116 & Gala1-3Galb1-4GIcNAcb-Sp8 & 1572 & 52 & 3 \\
\hline 37 & (3S)Galb1-4GlcNAcb-Sp8 & 1343 & 207 & 15 \\
\hline 574 & Neu5Aca2-8Neu5Aca2-3Galb1-3GalNAcb1-4(Neu5Aca2-3)Galb1-4GIc-Sp21 & 1192 & 137 & 11 \\
\hline 34 & (3S)Galb1-4(6S)GIcNAcb-Sp0 & 826 & 53 & 6 \\
\hline 27 & (3S)Galb1-4(6S)Glcb-Sp8 & 822 & 258 & 31 \\
\hline 318 & Neu5Aca2-3Galb1-4GIcNAcb1-6(Neu5Aca2-3Galb1-3)GalNAca-Sp14 & 815 & 49 & 6 \\
\hline 554 & Neu5Aca2-8Neu5Gca2-3Galb1-4GIcNAc-Sp0 & 791 & 123 & 16 \\
\hline 36 & (3S)Galb1-4GIcNAcb-Sp0 & 656 & 674 & 103 \\
\hline 26 & (3S)Galb1-4(6S)Glcb-Sp0 & 650 & 113 & 17 \\
\hline 207 & KDNa2-3Galb1-4GIcNAcb-Sp0 & 619 & 58 & 9 \\
\hline 296 & Neu5Aca2-3Galb1-4GIcNAcb1-3Galb1-3GIcNAcb-Sp0 & 532 & 140 & 26 \\
\hline 551 & $\begin{array}{l}\text { Galb1-4GlcNAcb1-3Galb1-4GIcNAcb1-3Galb1-4GlcNAcb1-3Galb1-4GlcNAcb1-2Mana1-6(Galb1-4GIcNAcb1- } \\
\text { 3Galb1-4GIcNAcb1-3Galb1-4GIcNAcb1-3Galb1-4GIcNAcb1-2Mana1-3)Manb1-4GlcNAcb1-4GIcNAcb-Sp25 }\end{array}$ & 443 & 140 & 32 \\
\hline 468 & Gala1-3(Fuca1-2)Galb1-3GalNAca-Sp8 & 438 & 37 & 8 \\
\hline 442 & Neu5Aca2-3Galb1-4GIcNAcb1-3Galb-Sp8 & 438 & 62 & 14 \\
\hline 553 & Neu5Gca2-8Neu5Gca2-3Galb1-4GIcNAc-Sp0 & 406 & 110 & 27 \\
\hline 118 & Gala1-3Galb1-4Glc-Sp10 & 376 & 74 & 20 \\
\hline 558 & Neu5Aca2-8Neu5Aca2-3Galb1-4GIcNAc-Sp0 & 354 & 36 & 10 \\
\hline 601 & Neu5Aca2-3Galb1-4GIcNAcb1-3Galb1-4GIcNAcb1-6(Galb1-3)GalNAca-Sp14 & 349 & 18 & 5 \\
\hline 490 & Gala1-3Galb1-3GIcNAcb1-6GalNAca-Sp14 & 319 & 38 & 12 \\
\hline 585 & $\begin{array}{l}\text { Galb1-4GIcNAcb1-3Galb1-4GIcNAcb1-3Galb1-4GIcNAcb1-6(Galb1-4GIcNAcb1-3Galb1-4GIcNAcb1-3Galb1- } \\
\text { 4GIcNAb1-2)Mana1-6(Galb1-4GlcNAcb1-3Galb1-4GlcNAcb1-3Galb1-4GlcNAcb1-2Mana1-3)Manb1-4GlcNAcb1- } \\
\text { 4(Fuca1-6)GlcNAcb-Sp24 }\end{array}$ & 271 & 251 & 93 \\
\hline 319 & $\begin{array}{l}\text { Neu5Aca2-6Galb1-4GIcNAcb1-2Mana1-6(Neu5Aca2-3Galb1-4GIcNAcb1-2Mana1-3)Manb1-4GIcNAcb1- } \\
\text { 4GIcNAcb-Sp12 }\end{array}$ & 249 & 39 & 16 \\
\hline 555 & Neu5Gca2-8Neu5Aca2-3Galb1-4GlcNAc-Sp0 & 229 & 83 & 36 \\
\hline
\end{tabular}




\begin{tabular}{|c|c|c|c|c|}
\hline 587 & $\begin{array}{l}\text { Galb1-4GIcNAcb1-3Galb1-4GIcNAcb1-3Galb1-4GIcNAcb1-3Galb1-4GIcNAcb1-6(Galb1-4GIcNAcb1-3Galb1- } \\
\text { 4GIcNAcb1-3Galb1-4GIcNAcb1-3Galb1-4GIcNAb1-2)Mana1-6(Galb1-4GIcNAcb1-3Galb1-4GIcNAcb1-3Galb1- } \\
\text { 4GIcNAcb1-3Galb1-4GIcNAcb1-2Mana1-3)Manb1-4GIcNAcb1-4(Fuca1-6)GIcNAcb-Sp24 }\end{array}$ & 226 & 66 & 29 \\
\hline 259 & Neu5Aca2-3Galb1-4GIcNAcb1-3Galb1-4GIcNAcb1-3Galb1-4GIcNAcb-Sp0 & 215 & 17 & 8 \\
\hline 35 & (3S)Galb1-4(6S)GIcNAcb-Sp8 & 209 & 74 & 36 \\
\hline 544 & $\begin{array}{l}\text { Neu5Gca2-3Galb1-4GIcNAcb1-3Galb1-4GIcNAcb1-2Mana1-6(Neu5Gca2-3Galb1-4GlcNAcb1-3Galb1- } \\
\text { 4GIcNAcb1-2Mana1-3)Manb1-4GIcNAcb1-4GIcNAcb-Sp24 }\end{array}$ & 208 & 29 & 14 \\
\hline 522 & Gala1-3Galb1-4GlcNAcb1-2Mana-Sp0 & 199 & 37 & 19 \\
\hline 277 & Neu5Aca2-8Neu5Aca2-3Galb1-4GIcb-Sp0 & 124 & 30 & 24 \\
\hline 406 & Gala1-3Galb1-4GIcNAcb1-3GalNAca-Sp14 & 119 & 34 & 28 \\
\hline 586 & $\begin{array}{l}\text { GlcNAcb1-3Galb1-4GlcNAcb1-3Galb1-4GIcNAcb1-3Galb1-4GlcNAcb1-6(GlcNAcb1-3Galb1-4GlcNAcb1-3Galb1- } \\
\text { 4GlcNAcb1-3Galb1-4GlcNAb1-2)Mana1-6(GIcNAcb1-3Galb1-4GlcNAcb1-3Galb1-4GlcNAcb1-3Galb1- } \\
\text { 4GIcNAcb1-2Mana1-3)Manb1-4GlcNAcb1-4(Fuca1-6)GlcNAcb-Sp24 }\end{array}$ & 113 & 128 & 113 \\
\hline 362 & Fuca1-2Galb1-4GIcNAcb1-2Mana1-6(Fuca1-2Galb1-4GIcNAcb1-2Mana1-3)Manb1-4GIcNAcb1-4GIcNAcb-Sp20 & 113 & 44 & 39 \\
\hline 165 & Galb1-4GlcNAcb1-3Galb1-4Glcb-Sp0 & 97 & 18 & 18 \\
\hline 252 & Neu5Aca2-3Galb1-4(6S)GlcNAcb-Sp8 & 92 & 11 & 12 \\
\hline 354 & Galb1-4GIcNAcb1-2Mana1-6(Galb1-4GIcNAcb1-2Mana1-3)Manb1-4GlcNAcb1-4(Fuca1-6)GlcNAcb-Sp22 & 90 & 69 & 77 \\
\hline 25 & (3S)Galb1-4Glcb-Sp8 & 90 & 39 & 44 \\
\hline 235 & Neu5Aca2-3Galb1-3GalNAcb1-4(Neu5Aca2-3)Galb1-4Glcb-Sp0 & 89 & 99 & 111 \\
\hline 597 & $\begin{array}{l}\text { Neu5Aca2-3Galb1-4GIcNAcb1-3Galb1-4GIcNAcb1-6(Neu5Aca2-3Galb1-4GIcNAcb1-3Galb1-4GIcNAcb1- } \\
\text { 3)GalNAca-Sp14 }\end{array}$ & 88 & 24 & 28 \\
\hline 573 & $\begin{array}{l}\text { Galb1-3GlcNAcb1-3Galb1-4GIcNAcb1-6(Galb1-3GlcNAcb1-3Galb1-4GlcNAb1-2)Mana1-6(Galb1-3GlcNAcb1- } \\
\text { 3Galb1-4GlcNAcb1-2Mana1-3)Manb1-4GlcNAcb1-4(Fuca1-6)GIcNAcb-Sp24 }\end{array}$ & 86 & 33 & 39 \\
\hline 243 & Neu5Aca2-3Galb1-3(6S)GalNAca-Sp8 & 84 & 28 & 33 \\
\hline 486 & $\begin{array}{l}\text { Galb1-4GlcNAcb1-6(Galb1-4GIcNAcb1-2)Mana1-6(Galb1-4GIcNAcb1-2Mana1-3)Manb1-4GlcNAcb1-4(Fuca1- } \\
\text { 6)GlcNAcb-Sp24 }\end{array}$ & 79 & 57 & 72 \\
\hline 572 & $\begin{array}{l}\text { Galb1-3GIcNAcb1-3Galb1-4GlcNAcb1-3Galb1-4GIcNAcb1-6(Galb1-3GlcNAcb1-3Galb1-4GIcNAcb1-3Galb1- } \\
\text { 4GIcNAb1-2)Mana1-6(Galb1-3GIcNAcb1-3Galb1-4GIcNAcb1-3Galb1-4GIcNAcb1-2Mana1-3)Manb1-4GlcNAcb1- } \\
\text { 4(Fuca1-6)GlcNAcb-Sp24 }\end{array}$ & 77 & 11 & 14 \\
\hline 459 & $\begin{array}{l}\text { Galb1-4GIcNAcb1-6(Galb1-4GIcNAcb1-2)Mana1-6(Galb1-4GIcNAcb1-2Mana1-3)Manb1-4GIcNAcb1-4GlcNAcb- } \\
\text { Sp19 }\end{array}$ & 72 & 22 & 31 \\
\hline 583 & $\begin{array}{l}\text { Galb1-4GIcNAcb1-3Galb1-4GIcNAcb1-6(Galb1-4GIcNAcb1-3Galb1-4GIcNAb1-2)Mana1-6(Galb1-4GIcNAcb1- } \\
\text { 3Galb1-4GIcNAcb1-2Mana1-3)Manb1-4GIcNAcb1-4(Fuca1-6)GIcNAcb-Sp24 }\end{array}$ & 70 & 24 & 34 \\
\hline 437 & $\begin{array}{l}\text { Galb1-4GIcNAcb1-2Mana1-6(GIcNAcb1-4)(Galb1-4GIcNAcb1-4(Galb1-4GIcNAcb1-2)Mana1-3)Manb1- } \\
\text { 4GlcNAcb1-4GlcNAc-Sp21 }\end{array}$ & 69 & 49 & 71 \\
\hline 301 & Galb1-3Galb1-4GIcNAcb-Sp8 & 65 & 27 & 42 \\
\hline 395 & Gala1-3Galb1-3GIcNAcb1-2Mana1-6(Gala1-3Galb1-3GIcNAcb1-2Mana1-3)Manb1-4GIcNAcb1-4GIcNAc-Sp19 & 64 & 53 & 82 \\
\hline 509 & $\begin{array}{l}\text { Galb1-4GIcNAcb1-6(Galb1-4GIcNAcb1-2)Mana1-6(GlcNAcb1-4)Galb1-4GlcNAcb1-4(Gal b1-4GIcNAcb1- } \\
\text { 2)Mana1-3)Manb1-4GIcNAcb1-4(Fuca1-6)GlcNAc-Sp21 }\end{array}$ & 63 & 44 & 69 \\
\hline 388 & $\begin{array}{l}\text { Galb1-4GIcNAcb1-6(Galb1-4GlcNAcb1-2)Mana1-6(Galb1-4GlcNAcb1-4(Galb1-4GlcNAcb1-2)Mana1-3)Manb1- } \\
\text { 4GlcNAcb1-4GlcNAcb-Sp21 }\end{array}$ & 59 & 16 & 27 \\
\hline 115 & Gala1-3Galb1-3GlcNAcb-Sp0 & 58 & 11 & 20 \\
\hline
\end{tabular}




\begin{tabular}{|c|c|c|c|c|}
\hline 582 & $\begin{array}{l}\text { Galb1-4GIcNAcb1-3Galb1-4GIcNAcb1-3Galb1-4GlcNAcb1-3Galb1-4GlcNAcb1-3Galb1-4GIcNAcb1-2Mana1- } \\
\text { 6(Galb1-4GIcNAcb1-3Galb1-4GIcNAcb1-3Galb1-4GIcNAcb1-3Galb1-4GlcNAcb1-3Galb1-4GIcNAcb1-2Mana1- } \\
\text { 3)Manb1-4GlcNAcb1-4(Fuca1-6)GIcNAcb-Sp19 }\end{array}$ & 56 & 19 & 34 \\
\hline 372 & $\begin{array}{l}\text { Gala1-3(Fuca1-2)Galb1-4GlcNAcb1-2Mana1-6(Gala1-3(Fuca1-2)Galb1-4GIcNAcb1-2Mana1-3)Manb1- } \\
\text { 4GlcNAcb1-4GlcNAcb-Sp20 }\end{array}$ & 54 & 10 & 18 \\
\hline 133 & Galb1-4GIcNAcb1-6GalNAc-Sp14 & 54 & 6 & 12 \\
\hline 568 & Galb1-3GlcNAcb1-6(Galb1-3)GalNAc-Sp14 & 52 & 13 & 26 \\
\hline 338 & Neu5Aca2-3Galb1-4(Fuca1-3)GlcNAcb1-6(Neu5Aca2-3Galb1-3)GalNAc-Sp14 & 50 & 25 & 50 \\
\hline 439 & $\begin{array}{l}\text { Galb1-4GlcNAcb1-6(Galb1-4GlcNAcb1-2)Mana1-6(GlcNAcb1-4)(Galb1-4GlcNAcb1-4(Galb1-4GlcNAcb1- } \\
\text { 2)Mana1-3)Manb1-4GlcNAcb1-4GIcNAc-Sp21 }\end{array}$ & 49 & 6 & 11 \\
\hline 327 & $\begin{array}{l}\text { Neu5Aca2-3Galb1-4GIcNAcb1-2Mana1-6(Neu5Aca2-6Galb1-4GIcNAcb1-2Mana1-3)Manb1-4GIcNAcb1- } \\
\text { 4GIcNAcb-Sp12 }\end{array}$ & 49 & 14 & 30 \\
\hline 379 & Neu5Aca2-3Galb1-4(Fuca1-3)GlcNAcb1-3GalNAca-Sp14 & 48 & 19 & 39 \\
\hline 534 & Fuca1-4(Galb1-3)GIcNAcb1-2 Mana-Sp0 & 47 & 8 & 17 \\
\hline 357 & KDNa2-3Galb1-4(Fuca1-3)GIcNAc-Sp0 & 47 & 24 & 50 \\
\hline 538 & Gala1-3(Fuca1-2)Galb1-3GalNAcb1-3Gala1-4Galb1-4Glc-Sp21 & 47 & 11 & 22 \\
\hline 532 & $\begin{array}{l}\text { Galb1-4GIcNAcb1-2 Mana1-6(GIcNAcb1-4)(Galb1-4GIcNAcb1-2Mana1-3)Manb1-4GIcNAcb1-4(Fuca1-6)GlcNAc- } \\
\text { Sp21 }\end{array}$ & 46 & 6 & 12 \\
\hline 334 & Gala1-4Galb1-4GIcNAcb1-3Galb1-4GIcb-Sp0 & 45 & 20 & 44 \\
\hline 511 & Galb1-3(6S)GlcNAcb-Sp8 & 45 & 18 & 41 \\
\hline 396 & $\begin{array}{l}\text { Gala1-3Galb1-3(Fuca1-4)GlcNAcb1-2Mana1-6(Gala1-3Galb1-3(Fuca1-4)GlcNAcb1-2Mana1-3)Manb1- } \\
\text { 4GlcNAcb1-4GlcNAc-Sp19 }\end{array}$ & 45 & 54 & 120 \\
\hline 503 & Neu5Aca2-6GalNAcb1-4(6S)GIcNAcb-Sp8 & 43 & 36 & 85 \\
\hline 542 & $\begin{array}{l}\text { Galb1-4GIcNAcb1-3Galb1-4GIcNAcb1-2Mana1-6(Galb1-4GIcNAcb1-3Galb1-4GIcNAcb1-2Mana1-3)Manb1- } \\
\text { 4GIcNAcb1-4GlcNAcb-Sp12 }\end{array}$ & 43 & 18 & 43 \\
\hline 376 & $\begin{array}{l}\text { Fuca1-4(Fuca1-2Galb1-3)GlcNAcb1-2Mana1-3(Fuca1-4(Fuca1-2Galb1-3)GlcNAcb1-2Mana1-3)Manb1- } \\
\text { 4GlcNAcb1-4GlcNAcb-Sp19 }\end{array}$ & 42 & 13 & 30 \\
\hline 546 & $\begin{array}{l}\text { GlcNAcb1-3Galb1-4GlcNAcb1-3Galb1-4GIcNAcb1-2Mana1-6(GlcNAcb1-3Galb1-4GlcNAcb1-3Galb1-4GlcNAcb1- } \\
\text { 2Mana1-3)Manb1-4GIcNAcb1-4GlcNAcb-Sp12 }\end{array}$ & 41 & 15 & 36 \\
\hline 569 & $\begin{array}{l}\text { Galb1-4GIcNAcb1-3Galb1-4GIcNAcb1-3Galb1-4GIcNAcb1-3Galb1-4GlcNAcb1-3Galb1-4GlcNAcb1-3Galb1- } \\
\text { 4GlcNAcb1-2Mana1-6(Galb1-4GlcNAcb1-3Galb1-4GlcNAcb1-3Galb1-4GlcNAcb1-3Galb1-4GlcNAcb1-3Galb1- } \\
\text { 4GlcNAcb1-3Galb1-4GIcNAcb1-2Mana1-3)Manb1-4GlcNAcb1-4GlcNAcb-Sp25 }\end{array}$ & 41 & 7 & 17 \\
\hline 506 & Fuca1-2Galb1-3GIcNAcb1-6(Fuca1-2Galb1-3GIcNAcb1-3)GalNAca-Sp14 & 41 & 13 & 32 \\
\hline 343 & GlcNAca1-4Galb1-4GIcNAcb1-3Galb1-4(Fuca1-3)GIcNAcb1-3Galb1-4(Fuca1-3)GIcNAcb-Sp0 & 41 & 12 & 30 \\
\hline 545 & $\begin{array}{l}\text { Fuca1-2Galb1-4GIcNAcb1-3Galb1-4GIcNAcb1-2Mana1-6(Fuca1-2Galb1-4GIcNAcb1-3Galb1-4GIcNAcb1- } \\
\text { 2Mana1-3)Manb1-4GIcNAcb1-4GIcNAcb-Sp24 }\end{array}$ & 40 & 9 & 22 \\
\hline 413 & Neu5Aca2-3Galb1-3GalNAcb1-4(Neu5Aca2-8Neu5Aca2-3)Galb1-4Glcb-Sp0 & 40 & 27 & 69 \\
\hline 519 & (6P)Galb1-4GlcNAcb-SP0 & 40 & 19 & 48 \\
\hline 449 & Galb1-4GIcNAcb1-2Mana-Sp0 & 39 & 22 & 56 \\
\hline 533 & $\begin{array}{l}\text { Galb1-4GlcNAcb1-2 Mana1-6(Galb1-4GlcNAcb1-4)(Galb1-4GlcNAcb1-2Mana1-3)Manb1-4GlcNAcb1-4(Fuca1- } \\
\text { 6)GlcNAc-Sp21 }\end{array}$ & 39 & 22 & 56 \\
\hline 248 & Neu5Aca2-3Galb1-3GIcNAcb1-3Galb1-4GIcNAcb-Sp0 & 39 & 14 & 36 \\
\hline 19 & Galb1-4GIcNAcb1-6(Galb1-4GlcNAcb1-3)GalNAca-Sp8 & 39 & 23 & 59 \\
\hline
\end{tabular}




\begin{tabular}{|c|c|c|c|c|}
\hline 450 & Fuca1-2Galb1-4GIcNAcb1-6(Fuca1-2Galb1-4GIcNAcb1-3)GalNAc-Sp14 & 39 & 22 & 57 \\
\hline 320 & Galb1-4GIcNAcb1-2Mana1-6(Neu5Aca2-6Galb1-4GIcNAcb1-2Mana1-3)Manb1-4GIcNAcb1-4GlcNAcb-Sp12 & 39 & 14 & 36 \\
\hline 193 & GlcNAcb1-6GalNAca-Sp8 & 39 & 19 & 49 \\
\hline 356 & (6S)GlcNAcb1-3Galb1-4GlcNAcb-Sp0 & 39 & 17 & 44 \\
\hline 239 & Neu5Aca2-3Galb1-3(6S)GIcNAc-Sp8 & 38 & 3 & 9 \\
\hline 483 & $\begin{array}{l}\text { Neu5Aca2-6Galb1-4GIcNAcb1-2Mana1-6(Neu5Aca2-6Galb1-4GIcNAcb1-2Mana1-3)Manb1-4GIcNAcb1-4(Fuca1- } \\
\text { 6)GIcNAcb-Sp24 }\end{array}$ & 38 & 20 & 53 \\
\hline 421 & $\begin{array}{l}\text { Fuca1-2Galb1-4GIcNAcb1-2Mana1-6(Fuca1-2Galb1-4GIcNAcb1-2Mana1-3)Manb1-4GIcNAcb1-4(Fuca1- } \\
\text { 6)GIcNAcb-Sp22 }\end{array}$ & 38 & 18 & 48 \\
\hline 81 & Fuca1-4GIcNAcb-Sp8 & 38 & 20 & 52 \\
\hline 446 & $\begin{array}{l}\text { Fuca1-2Galb1-4 GlcNAcb1-2Mana1-6(Fuca1-2Galb1-4GIcNAcb1-2(Fuca1-2Galb1-4GlcNAcb1-4)Mana1- } \\
\text { 3)Manb1-4GIcNAcb1-4GIcNAcb-Sp12 }\end{array}$ & 38 & 9 & 24 \\
\hline 225 & Neu5Aca2-3Galb1-3GalNAca-Sp14 & 38 & 20 & 52 \\
\hline 605 & GlcNAcb1-6(Neu5Aca2-3Galb1-3)GalNAca-Sp14 & 37 & 17 & 44 \\
\hline 472 & Neu5Aca2-3Galb1-4GIcNAcb1-6(Neu5Aca2-3Galb1-4GlcNAcb1-3)GalNAca-Sp14 & 37 & 23 & 62 \\
\hline 495 & Fuca1-2Galb1-4GlcNAcb1-6GalNAca-Sp14 & 37 & 15 & 40 \\
\hline 31 & (3S)Galb1-3GlcNAcb-Sp8 & 37 & 19 & 52 \\
\hline 419 & GalNAca1-3(Fuca1-2)Galb1-4(Fuca1-3)GlcNAcb1-3GalNAc-Sp14 & 37 & 14 & 39 \\
\hline 280 & Neu5Acb2-6Galb1-4GIcNAcb-Sp8 & 37 & 9 & 24 \\
\hline 345 & GlcNAca1-4Galb1-3GalNAc-Sp14 & 37 & 15 & 41 \\
\hline 82 & Fucb1-3GlcNAcb-Sp8 & 36 & 18 & 50 \\
\hline 417 & Fuca1-2Galb1-4(Fuca1-3)GlcNAcb1-3GalNAca-Sp14 & 36 & 36 & 98 \\
\hline 328 & $\begin{array}{l}\text { Galb1-4(Fuca1-3)GIcNAcb1-2Mana1-6(Galb1-4(Fuca1-3)GIcNAcb1-2Mana1-3)Manb1-4GIcNAcb1-4GIcNAcb- } \\
\text { Sp20 }\end{array}$ & 36 & 8 & 21 \\
\hline 11 & Neu5Acb-Sp8 & 36 & 3 & 8 \\
\hline 286 & Neu5Gca2-6GalNAca-Sp0 & 36 & 17 & 48 \\
\hline 58 & $\begin{array}{l}\text { Neu5Aca2-6Galb1-4GlcNAcb1-2Mana1-6(Neu5Aca2-6Galb1-4GlcNAcb1-2Mana1-3)Manb1-4GIcNAcb1- } \\
\text { 4GIcNAcb-Sp24 }\end{array}$ & 36 & 13 & 35 \\
\hline 578 & $\begin{array}{l}\text { Galb1-4GIcNAcb1-3Galb1-4GIcNAcb1-3Galb1-4GIcNAcb1-2Mana1-6(Galb1-4GIcNAcb1-3Galb1-4GlcNAcb1- } \\
\text { 3Galb1-4GIcNAcb1-2Mana1-3)Manb1-4GlcNAcb1-4(Fuca1-6)GIcNAcb-Sp24 }\end{array}$ & 35 & 14 & 40 \\
\hline 436 & Galb1-4GIcNAcb1-2Mana1-6(GlcNAcb1-4)(Galb1-4GIcNAcb1-2Mana1-3)Manb1-4GIcNAcb1-4GIcNAc-Sp21 & 35 & 20 & 57 \\
\hline 377 & Neu5Aca2-3Galb1-4GIcNAcb1-3GalNAc-Sp14 & 35 & 33 & 93 \\
\hline 273 & Neu5Aca2-6Galb1-4GIcb-Sp0 & 35 & 16 & 45 \\
\hline 292 & Galb1-4(Fuca1-3)(6S)Glcb-Sp0 & 35 & 26 & 73 \\
\hline 140 & Galb1-3GalNAca-Sp8 & 35 & 33 & 94 \\
\hline 384 & Galb1-4(Fuca1-3)GlcNAcb1-6(Galb1-3GlcNAcb1-3)Galb1-4Glc-Sp21 & 35 & 9 & 26 \\
\hline 73 & Fuca1-2Galb1-4(Fuca1-3)GlcNAcb-Sp8 & 35 & 15 & 43 \\
\hline 608 & $\begin{array}{l}\text { Neu5Aca2-3Galb1-4GIcNAcb1-3Galb1-4GIcNAcb1-3Galb1-4GlcNAcb1-2Mana1-6(Neu5Aca2-3Galb1- } \\
\text { 4GIcNAcb1-3Galb1-4GIcNAcb1-3Galb1-4GIcNAcb1-2Mana1-3)Manb1-4GlcNAcb1-4GlcNAcb-Sp12 }\end{array}$ & 35 & 4 & 12 \\
\hline 110 & Gala1-4(Gala1-3)Galb1-4GIcNAcb-Sp8 & 35 & 22 & 63 \\
\hline 45 & (6S)Galb1-4(6S)Glcb-Sp8 & 35 & 16 & 45 \\
\hline 548 & $\begin{array}{l}\text { Galb1-4GIcNAcb1-3Galb1-4GlcNAcb1-3Galb1-4GlcNAcb1-2Mana1-6(Galb1-4GlcNAcb1-3Galb1-4GlcNAcb1- } \\
\text { 3Galb1-4GIcNAcb1-2Mana1-3)Manb1-4GlcNAcb1-4GIcNAcb-Sp12 }\end{array}$ & 34 & 5 & 15 \\
\hline
\end{tabular}




\begin{tabular}{|c|c|c|c|c|}
\hline 147 & Galb1-3Galb-Sp8 & 34 & 16 & 48 \\
\hline 470 & Glca1-6Glca1-6Glca1-6Glcb-Sp10 & 34 & 31 & 89 \\
\hline 447 & $\begin{array}{l}\text { Fuca1-2Galb1-4(Fuca1-3)GIcNAcb1-2Mana1-6(Fuca1-2Galb1-4(Fuca1-3)GIcNAcb1-4(Fuca1-2Galb1-4(Fuca1- } \\
\text { 3)GIcNAcb1-2)Mana1-3)Manb1-4GIcNAcb1-4GIcNAcb-Sp12 }\end{array}$ & 34 & 20 & 59 \\
\hline 151 & Galb1-3GIcNAcb-Sp8 & 34 & 24 & 70 \\
\hline 607 & $\begin{array}{l}\text { Neu5Aca2-6Galb1-4GIcNAcb1-3Galb1-4GIcNAcb1-3Galb1-4GIcNAcb1-2Mana1-6(Neu5Aca2-6Galb1- } \\
\text { 4GIcNAcb1-3Galb1-4GIcNAcb1-3Galb1-4GIcNAcb1-2Mana1-3)Manb1-4GIcNAcb1-4GlcNAcb-Sp12 }\end{array}$ & 34 & 11 & 31 \\
\hline 385 & Galb1-4GIcNAcb1-6(Fuca1-4(Fuca1-2Galb1-3)GIcNAcb1-3)Galb1-4Glc-Sp21 & 34 & 22 & 66 \\
\hline 361 & Fuca1-2Galb1-3GlcNAcb1-2Mana1-6(Fuca1-2Galb1-3GlcNAcb1-2Mana1-3)Manb1-4GlcNAcb1-4GlcNAcb-Sp20 & 34 & 19 & 57 \\
\hline 203 & GlcAb-Sp8 & 33 & 10 & 30 \\
\hline 117 & Gala1-3Galb1-4GIcb-Sp0 & 33 & 12 & 36 \\
\hline 174 & GlcNAca1-3Galb1-4GlcNAcb-Sp8 & 33 & 9 & 26 \\
\hline 312 & GIcNAcb1-4GlcNAcb-Sp12 & 33 & 29 & 89 \\
\hline 373 & $\begin{array}{l}\text { Gala1-3Galb1-4(Fuca1-3)GlcNAcb1-2Mana1-6(Gala1-3Galb1-4(Fuca1-3)GIcNAcb1-2Mana1-3)Manb1- } \\
\text { 4GIcNAcb1-4GIcNAcb-Sp20 }\end{array}$ & 33 & 6 & 19 \\
\hline 496 & Gala1-3Galb1-4GIcNAcb1-6GalNAca-Sp14 & 33 & 7 & 21 \\
\hline 510 & Galb1-3GIcNAca1-3Galb1-4GIcNAcb-Sp8 & 32 & 7 & 23 \\
\hline 41 & (6P)Mana-Sp8 & 32 & 5 & 17 \\
\hline 598 & Neu5Aca2-6Galb1-4GIcNAcb1-3Galb1-4GlcNAcb1-3GalNAca-Sp14 & 32 & 17 & 54 \\
\hline 399 & Galb1-4GIcNAcb1-2Mana1-6(GIcNAcb1-2Mana1-3)Manb1-4GIcNAcb1-4GIcNAc-Sp12 & 32 & 28 & 89 \\
\hline 560 & $\begin{array}{l}\text { Galb1-4GIcNAcb1-3Galb1-4GIcNAcb1-6(Galb1-4GIcNAcb1-3Galb1-4GIcNAcb1-2)Mana1-6(Galb1-4GIcNAcb1- } \\
\text { 3Galb1-4GIcNAcb1-2Mana1-3)Mana1-4GIcNAcb1-4GIcNAc-Sp24 }\end{array}$ & 32 & 13 & 39 \\
\hline 591 & Galb1-4GlcNAcb1-3Galb1-4GlcNAcb1-6(Galb1-3)GalNAca-Sp14 & 32 & 26 & 82 \\
\hline 562 & GlcNAcb1-3Galb1-4GlcNAcb1-6(GlcNAcb1-3Galb1-3)GalNAca-Sp14 & 32 & 10 & 33 \\
\hline 595 & GlcNAcb1-3Galb1-4GlcNAcb1-6(Galb1-3)GalNAca-Sp14 & 32 & 19 & 61 \\
\hline 98 & GalNAcb1-4(Fuca1-3)GlcNAcb-Sp0 & 32 & 24 & 75 \\
\hline 440 & Galb1-4Galb-Sp10 & 32 & 12 & 40 \\
\hline 604 & $\begin{array}{l}\text { Neu5Aca2-3Galb1-4GIcNAcb1-3Galb1-4GlcNAcb1-2Mana1-6(Neu5Aca2-3Galb1-4GIcNAcb1-3Galb1- } \\
\text { 4GIcNAcb1-2Mana1-3)Manb1-4GIcNAcb1-4GlcNAcb-Sp12 }\end{array}$ & 32 & 7 & 23 \\
\hline 526 & Gala1-3(Fuca1-2)Galb1-3GlcNAcb1-6GalNAc-Sp14 & 31 & 17 & 55 \\
\hline 47 & (6S)GlcNAcb-Sp8 & 31 & 17 & 55 \\
\hline 366 & $\begin{array}{l}\text { Fuca1-4(Galb1-3)GIcNAcb1-2Mana1-6(Fuca1-4(Galb1-3)GIcNAcb1-2Mana1-3)Manb1-4GlcNAcb1-4(Fuca1- } \\
\text { 6)GIcNAcb-Sp22 }\end{array}$ & 31 & 12 & 38 \\
\hline 469 & Gala1-3(Fuca1-2)Galb1-3GalNAcb-Sp8 & 31 & 12 & 40 \\
\hline 245 & Neu5Aca2-6(Neu5Aca2-3Galb1-3)GalNAca-Sp14 & 31 & 10 & 33 \\
\hline 310 & GlcNAcb1-3Man-Sp10 & 31 & 22 & 71 \\
\hline 156 & Galb1-4(6S)Glcb-Sp0 & 31 & 2 & 7 \\
\hline 265 & Neu5Aca2-3Galb1-4Glcb-Sp8 & 31 & 11 & 36 \\
\hline 303 & Galb1-4GIcNAcb1-6(Galb1-4GIcNAcb1-3)Galb1-4GIcNAc-Sp0 & 31 & 13 & 42 \\
\hline 30 & (3S)Galb1-3GlcNAcb-Sp0 & 30 & 8 & 27 \\
\hline 196 & Glca1-4Glcb-Sp8 & 30 & 6 & 18 \\
\hline 501 & Fuca1-2Galb1-3(6S)GIcNAcb-Sp0 & 30 & 19 & 64 \\
\hline 426 & Gala1-3Galb1-3GlcNAcb1-3GalNAc-Sp14 & 30 & 20 & 66 \\
\hline
\end{tabular}




\begin{tabular}{|c|c|c|c|c|}
\hline 285 & Neu5Gca2-3Galb1-4Glcb-Sp0 & 30 & 8 & 27 \\
\hline 226 & GalNAcb1-4(Neu5Aca2-8Neu5Aca2-8Neu5Aca2-8Neu5Aca2-3)Galb1-4Glcb-Sp0 & 30 & 18 & 59 \\
\hline 201 & G-ol-Sp8 & 30 & 21 & 70 \\
\hline 192 & GlcNAcb1-4GlcNAcb1-4GIcNAcb-Sp8 & 30 & 8 & 27 \\
\hline 370 & $\begin{array}{l}\text { Galb1-4GIcNAcb1-2Mana1-6(Galb1-4GIcNAcb1-4(Galb1-4GIcNAcb1-2)Mana1-3)Manb1-4GIcNAcb1-4GIcNAc- } \\
\text { Sp21 }\end{array}$ & 30 & 12 & 41 \\
\hline 107 & Gala1-3(Fuca1-2)Galb1-4GIcb-Sp0 & 30 & 4 & 15 \\
\hline 456 & $\begin{array}{l}\text { Gala1-3(Fuca1-2)Galb1-3GlcNAcb1-2Mana1-6(Gala1-3(Fuca1-2)Galb1-3GlcNAcb1-2Mana1-3)Manb1- } \\
\text { 4GlcNAcb1-4(Fuca1-6)GlcNAcb-Sp22 }\end{array}$ & 30 & 13 & 43 \\
\hline 64 & Fuca1-2Galb1-3GalNAcb1-4(Neu5Aca2-3)Galb1-4Glcb-Sp0 & 29 & 10 & 35 \\
\hline 454 & GalNAcb1-4Galb1-4GIcb-Sp0 & 29 & 14 & 48 \\
\hline 154 & Galb1-4(Fuca1-3)GlcNAcb1-3Galb1-4(Fuca1-3)GlcNAcb-Sp0 & 29 & 24 & 81 \\
\hline 261 & Neu5Aca2-3Galb1-4GlcNAcb-Sp8 & 29 & 25 & 87 \\
\hline 69 & Fuca1-2Galb1-3GIcNAcb-Sp8 & 29 & 25 & 88 \\
\hline 99 & GalNAcb1-4GlcNAcb-Sp0 & 29 & 15 & 51 \\
\hline 57 & $\begin{array}{l}\text { Neu5Aca2-6Galb1-4GIcNAcb1-2Mana1-6(Neu5Aca2-6Galb1-4GIcNAcb1-2Man-a1-3)Manb1-4GIcNAcb1- } \\
\text { 4GIcNAcb-Sp21 }\end{array}$ & 29 & 8 & 29 \\
\hline 302 & Neu5Aca2-6Galb1-4GIcNAcb1-2Mana1-6(Galb1-4GIcNAcb1-2Mana1-3)Manb1-4GIcNAcb1-4GIcNAcb-Sp12 & 29 & 30 & 103 \\
\hline 420 & $\begin{array}{l}\text { Galb1-4(Fuca1-3)GIcNAcb1-2Mana1-6(Galb1-4(Fuca1-3)GIcNAcb1-2Mana1-3)Manb1-4GIcNAcb1-4(Fuca1- } \\
\text { 6)GIcNAcb-Sp22 }\end{array}$ & 29 & 18 & 65 \\
\hline 260 & Neu5Aca2-3Galb1-4GlcNAcb-Sp0 & 29 & 13 & 46 \\
\hline 258 & Neu5Aca2-3Galb1-4(Fuca1-3)GlcNAcb1-3Galb1-4GIcNAcb-Sp8 & 29 & 6 & 22 \\
\hline 350 & Galb1-4GIcNAcb1-2Mana1-3Manb1-4GIcNAcb1-4GIcNAc-Sp12 & 28 & 14 & 51 \\
\hline 596 & GlcNAcb1-3Galb1-4GlcNAcb1-6(GlcNAcb1-3Galb1-4GIcNAcb1-3)GalNAca-Sp14 & 28 & 8 & 29 \\
\hline 217 & Mana1-6(Mana1-3)Mana1-6(Mana1-3)Manb1-4GIcNAcb1-4GIcNAcb-Sp12 & 28 & 16 & 58 \\
\hline 124 & Gala1-4GIcNAcb-Sp8 & 28 & 22 & 78 \\
\hline 8 & Rhaa-Sp8 & 28 & 12 & 42 \\
\hline 559 & $\begin{array}{l}\text { GlcNAcb1-3Galb1-4GlcNAcb1-6(GlcNAcb1-3Galb1-4GIcNAcb1-2)Mana1-6(GlcNAcb1-3Galb1-4GIcNAcb1- } \\
\text { 2Man a1-3)Manb1-4GIcNAcb1-4GIcNAc-Sp24 }\end{array}$ & 28 & 10 & 36 \\
\hline 371 & $\begin{array}{l}\text { GalNAca1-3(Fuca1-2)Galb1-4GlcNAcb1-2Mana1-6(GalNAca1-3(Fuca1-2)Galb1-4GIcNAcb1-2Mana1-3)Manb1- } \\
\text { 4GIcNAcb1-4GIcNAcb-Sp20 }\end{array}$ & 28 & 3 & 12 \\
\hline 279 & Neu5Acb2-6GalNAca-Sp8 & 28 & 11 & 39 \\
\hline 101 & Gala1-2Galb-Sp8 & 27 & 10 & 37 \\
\hline 476 & GlcNAcb1-6(GlcNAcb1-2)Mana1-6(GlcNAcb1-2Mana1-3)Manb1-4GIcNAcb1-4(Fuca1-6)GlcNAcb-Sp24 & 27 & 17 & 61 \\
\hline 169 & Galb1-4GIcNAcb-Sp0 & 27 & 20 & 75 \\
\hline 53 & GlcNAcb1-2Mana1-6(GlcNAcb1-2Mana1-3)Manb1-4GlcNAcb1-4GIcNAcb-Sp13 & 27 & 14 & 51 \\
\hline 557 & Neu5Gca2-8Neu5Gca2-6Galb1-4GIcNAc-Sp0 & 27 & 13 & 48 \\
\hline 75 & Fuca1-2Galb1-4GIcNAcb1-3Galb1-4GlcNAcb1-3Galb1-4GIcNAcb-Sp0 & 27 & 16 & 62 \\
\hline 315 & Mana1-6(Mana1-3)Mana1-6(Mana1-3)Manb-Sp10 & 27 & 12 & 45 \\
\hline 186 & GlcNAcb1-3Galb1-4Glcb-Sp0 & 27 & 17 & 64 \\
\hline 214 & Mana1-6(Mana1-3)Mana-Sp9 & 27 & 23 & 85 \\
\hline 331 & Neu5Aca2-6Galb1-4GIcNAcb1-3Galb1-3GIcNAcb-Sp0 & 27 & 9 & 34 \\
\hline
\end{tabular}




\begin{tabular}{|c|c|c|c|c|}
\hline 363 & $\begin{array}{l}\text { Fuca1-2Galb1-4(Fuca1-3)GlcNAcb1-2Mana1-6(Fuca1-2Galb1-4(Fuca1-3)GlcNAcb1-2Mana1-3)Manb1- } \\
\text { 4GlcNAcb1-4GlcNAb-Sp20 }\end{array}$ & 27 & 10 & 39 \\
\hline 435 & $\begin{array}{l}\text { GlcNAcb1-6(GlcNAcb1-2)Mana1-6(GlcNAcb1-4)(GlcNAcb1-4(GlcNAcb1-2)Mana1-3)Manb1-4GIcNAcb1- } \\
\text { 4GIcNAc-Sp21 }\end{array}$ & 26 & 6 & 24 \\
\hline 603 & Neu5Aca2-6Galb1-4GIcNAcb1-6(Galb1-3)GalNAca-Sp14 & 26 & 9 & 34 \\
\hline 393 & GalNAcb1-4(Neu5Aca2-3)Galb1-4GlcNAcb1-3GalNAca-Sp14 & 26 & 7 & 25 \\
\hline 121 & Gala1-4Galb1-4GIcNAcb-Sp0 & 26 & 15 & 57 \\
\hline 237 & Neu5Aca2-3GalNAca-Sp8 & 26 & 15 & 60 \\
\hline 91 & GalNAca1-3(Fuca1-2)Galb-Sp18 & 26 & 13 & 51 \\
\hline 528 & Gala1-3Galb1-3GlcNAcb1-2Mana-Sp0 & 26 & 13 & 49 \\
\hline 549 & $\begin{array}{l}\text { Galb1-4GlcNAcb1-3Galb1-4GlcNAcb1-3Galb1-4GlcNAcb1-2Mana1-6(Galb1-4GlcNAcb1-3Galb1-4GlcNAcb1- } \\
\text { 3Galb1-4GlcNAcb1-2Mana1-3)Manb1-4GlcNAcb1-4GlcNAcb-Sp24 }\end{array}$ & 26 & 10 & 39 \\
\hline 197 & Glca1-4Glca-Sp8 & 26 & 6 & 25 \\
\hline 102 & Gala1-3(Fuca1-2)Galb1-3GlcNAcb-Sp0 & 26 & 2 & 10 \\
\hline 606 & $\begin{array}{l}\text { Neu5Aca2-6Galb1-4GIcNAcb1-3Galb1-4GIcNAcb1-6(Neu5Aca2-6Galb1-4GIcNAcb1-3Galb1-4GIcNAcb1- } \\
\text { 3)GalNAca-Sp14 }\end{array}$ & 26 & 18 & 72 \\
\hline 552 & $\begin{array}{l}\text { Galb1-3GIcNAcb1-3Galb1-4GIcNAcb1-2Mana1-6(Galb1-3GlcNAcb1-3Galb1-4GIcNAcb1-2Mana1-3)Manb1- } \\
\text { 4GlcNAcb1-4GlcNAc-Sp25 }\end{array}$ & 26 & 7 & 29 \\
\hline 333 & Neu5Aca2-6Galb1-4GIcNAcb1-3Galb1-4GIcNAcb1-3Galb1-4GIcNAcb-Sp0 & 25 & 10 & 40 \\
\hline 67 & Fuca1-2Galb1-3GIcNAcb1-3Galb1-4Glcb-Sp10 & 25 & 8 & 33 \\
\hline 189 & GlcNAcb1-4Galb1-4GlcNAcb-Sp8 & 25 & 17 & 66 \\
\hline 300 & Neu5Aca2-3Galb1-4(Fuca1-3)GlcNAcb1-6(Galb1-3)GalNAca-Sp14 & 25 & 8 & 30 \\
\hline 126 & Galb1-2Galb-Sp8 & 25 & 15 & 59 \\
\hline 42 & (6S)Galb1-4GIcb-Sp0 & 25 & 13 & 52 \\
\hline 466 & $\begin{array}{l}\text { Neu5Aca2-6Galb1-4GlcNAcb1-6(Neu5Aca2-6Galb1-4GIcNAcb1-2)Mana1-6(GlcNAcb1-4)(Neu5Aca2-6Galb1- } \\
\text { 4GIcNAcb1-2Mana1-3)Manb1-4GlcNAcb1-4GlcNAcb-Sp21 }\end{array}$ & 25 & 11 & 47 \\
\hline 530 & Neu5Aca2-3Galb1-3GlcNAcb1-4Galb1-4Glcb-Sp0 & 25 & 11 & 44 \\
\hline 480 & Neu5Aca2-3Galb1-4GlcNAcb1-6GalNAca-Sp14 & 25 & 15 & 61 \\
\hline 246 & Neu5Aca2-3Galb-Sp8 & 24 & 11 & 46 \\
\hline 220 & (3S)Galb1-4(Fuca1-3)(6S)GlcNAcb-Sp8 & 24 & 7 & 30 \\
\hline 576 & $\begin{array}{l}\text { Galb1-4GIcNAcb1-3Galb1-4GIcNAcb1-2Mana1-6(Galb1-4GIcNAcb1-3Galb1-4GIcNAcb1-2Mana1-3)Manb1- } \\
\text { 4GIcNAcb1-4(Fuca1-6)GIcNAcb-Sp24 }\end{array}$ & 24 & 13 & 54 \\
\hline 324 & Neu5Gcb2-6Galb1-4GIcNAc-Sp8 & 24 & 22 & 90 \\
\hline 594 & GlcNAcb1-3Galb1-4GIcNAcb1-3GalNAca-Sp14 & 24 & 5 & 19 \\
\hline 329 & Neu5,9Ac2a2-3Galb1-4GlcNAcb-Sp0 & 24 & 7 & 28 \\
\hline 473 & $\begin{array}{l}\text { Fuca1-2Galb1-4(Fuca1-3)GlcNAcb1-2Mana1-6(Fuca1-2Galb1-4(Fuca1-3)GlcNAcb1-2Mana1-3)Manb1- } \\
\text { 4GlcNAcb1-4(Fuca1-6)GlcNAcb-Sp24 }\end{array}$ & 24 & 10 & 40 \\
\hline 241 & Neu5Aca2-3Galb1-3(Fuca1-4)GlcNAcb1-3Galb1-4(Fuca1-3)GIcNAcb-Sp0 & 24 & 19 & 81 \\
\hline 209 & Mana1-2Mana1-6(Mana1-2Mana1-3)Mana-Sp9 & 24 & 12 & 49 \\
\hline 565 & $\begin{array}{l}\text { GlcNAcb1-3Galb1-4GlcNAcb1-3Galb1-4GlcNAcb1-3Galb1-4GlcNAcb1-3Galb1-4GlcNAcb1-2Mana1-6(GlcNAcb1- } \\
\text { 3Galb1-4GIcNAcb1-3Galb1-4GIcNAcb1-3Galb1-4GIcNAcb1-3Galb1-4GlcNAcb1-2Mana1-3)Manb1-4GlcNAcb1- } \\
\text { 4GlcNAcb-Sp25 }\end{array}$ & 24 & 10 & 44 \\
\hline 111 & Gala1-3GalNAca-Sp8 & 24 & 21 & 90 \\
\hline
\end{tabular}




\begin{tabular}{|c|c|c|c|c|}
\hline 423 & Fuca1-2Galb1-3GIcNAcb1-3GalNAc-Sp14 & 24 & 9 & 38 \\
\hline 15 & GalNAcb-Sp8 & 24 & 12 & 50 \\
\hline 383 & Galb1-3GlcNAcb1-3Galb1-4GlcNAcb1-6(Galb1-3GlcNAcb1-3)Galb1-4GIcb-Sp0 & 24 & 10 & 42 \\
\hline 438 & $\begin{array}{l}\text { Galb1-4GIcNAcb1-6(Galb1-4GlcNAcb1-2)Mana1-6(GlcNAcb1-4)(Galb1-4GlcNAcb1-2Mana1-3)Manb1- } \\
\text { 4GIcNAcb1-4GlcNAc-Sp21 }\end{array}$ & 23 & 9 & 40 \\
\hline 284 & Neu5Gca2-3Galb1-4GIcNAcb-Sp0 & 23 & 12 & 50 \\
\hline 337 & GalNAca1-3(Fuca1-2)Galb1-4GIcNAcb1-3Galb1-4GlcNAcb1-3Galb1-4GIcNAcb-Sp0 & 23 & 11 & 48 \\
\hline 76 & Fuca1-2Galb1-4GlcNAcb-Sp0 & 23 & 8 & 33 \\
\hline 276 & Neu5Aca2-8Neu5Aca-Sp8 & 23 & 12 & 49 \\
\hline 411 & Galb1-4GIcNAcb1-6(Neu5Aca2-6Galb1-3GIcNAcb1-3)Galb1-4Glc-Sp21 & 23 & 13 & 56 \\
\hline 409 & GalNAcb1-3Gala1-6Galb1-4Glcb-Sp8 & 23 & 14 & 61 \\
\hline 418 & Gala1-3(Fuca1-2)Galb1-4(Fuca1-3)GIcNAcb1-3GalNAc-Sp14 & 23 & 7 & 28 \\
\hline 253 & Neu5Aca2-3Galb1-4(Fuca1-3)(6S)GlcNAcb-Sp8 & 23 & 6 & 24 \\
\hline 206 & KDNa2-3Galb1-3GIcNAcb-Sp0 & 23 & 7 & 32 \\
\hline 60 & Fuca1-2Galb1-3GalNAcb1-3Gala1-4Galb1-4GIcb-Sp9 & 23 & 18 & 76 \\
\hline 321 & GlcNAcb1-2Mana1-6(Neu5Aca2-6Galb1-4GIcNAcb1-2Mana1-3)Manb1-4GlcNAcb1-4GlcNAcb-Sp12 & 23 & 6 & 27 \\
\hline 96 & GalNAcb1-3(Fuca1-2)Galb-Sp8 & 23 & 15 & 66 \\
\hline 290 & Galb1-3GIcNAcb1-3Galb1-3GIcNAcb-Sp0 & 23 & 14 & 60 \\
\hline 256 & Neu5Aca2-3Galb1-4(Fuca1-3)GlcNAcb-Sp8 & 23 & 10 & 44 \\
\hline 493 & (3S)Galb1-3(Fuca1-4)GIcNAcb-Sp0 & 23 & 18 & 79 \\
\hline 108 & Gala1-3(Fuca1-2)Galb-Sp8 & 23 & 11 & 48 \\
\hline 10 & Neu5Aca-Sp11 & 23 & 13 & 56 \\
\hline 183 & GlcNAcb1-3Galb1-4GlcNAcb-Sp0 & 23 & 11 & 48 \\
\hline 575 & $\begin{array}{l}\text { GlcNAcb1-3Galb1-4GlcNAcb1-2Mana1-6(GlcNAcb1-3Galb1-4GlcNAcb1-2Mana1-3)Manb1-4GlcNAcb1-4(Fuca1- } \\
\text { 6)GlcNAcb-Sp24 }\end{array}$ & 23 & 7 & 32 \\
\hline 218 & Manb1-4GIcNAcb-Sp0 & 23 & 17 & 73 \\
\hline 529 & GalNAcb1-4GIcNAcb1-2Mana-Sp0 & 23 & 15 & 67 \\
\hline 71 & Fuca1-2Galb1-4(Fuca1-3)GlcNAcb1-3Galb1-4(Fuca1-3)GlcNAcb1-3Galb1-4(Fuca1-3)GlcNAcb-Sp0 & 23 & 12 & 53 \\
\hline 219 & Neu5Aca2-3Galb1-4GIcNAcb1-3Galb1-4(Fuca1-3)GIcNAcb-Sp0 & 23 & 3 & 14 \\
\hline 340 & GIcNAca1-4Galb1-4GIcNAcb-Sp0 & 23 & 2 & 9 \\
\hline 494 & Galb1-4(Fuca1-3)GlcNAcb1-6(Neu5Aca2-6(Neu5Aca2-3Galb1-3)GlcNAcb1-3)Galb1-4Glc-Sp21 & 23 & 7 & 31 \\
\hline 523 & Gala1-3(Fuca1-2)Galb1-4GIcNAcb1-2Mana-Sp0 & 23 & 5 & 24 \\
\hline 448 & Galb1-4(Fuca1-3)GlcNAcb1-6GalNAc-Sp14 & 23 & 10 & 45 \\
\hline 579 & $\begin{array}{l}\text { GlcNAcb1-3Galb1-4GIcNAcb1-3Galb1-4GIcNAcb1-3Galb1-4GIcNAcb1-2Mana1-6(GlcNAcb1-3Galb1-4GIcNAcb1- } \\
\text { 3Galb1-4GIcNAcb1-3Galb1-4GIcNAcb1-2Mana1-3)Manb1-4GIcNAcb1-4(Fuca1-6)GlcNAcb-Sp24 }\end{array}$ & 23 & 3 & 15 \\
\hline 46 & Neu5Aca2-3(6S)Galb1-4GIcNAcb-Sp8 & 23 & 7 & 31 \\
\hline 525 & Galb1-3GIcNAcb1-2Mana-Sp0 & 22 & 10 & 45 \\
\hline 541 & $\begin{array}{l}\text { GlcNAcb1-3Galb1-4GlcNAcb1-2Mana1-6(GlcNAcb1-3Galb1-4GlcNAcb1-2Mana1-3)Manb1-4GlcNAcb1- } \\
\text { 4GlcNAcb-Sp25 }\end{array}$ & 22 & 7 & 33 \\
\hline 77 & Fuca1-2Galb1-4GIcNAcb-Sp8 & 22 & 11 & 51 \\
\hline 120 & Gala1-4(Fuca1-2)Galb1-4GIcNAcb-Sp8 & 22 & 13 & 56 \\
\hline 412 & Galb1-3GalNAcb1-4(Neu5Aca2-8Neu5Aca2-3)Galb1-4Glcb-Sp0 & 22 & 11 & 50 \\
\hline 227 & GalNAcb1-4(Neu5Aca2-8Neu5Aca2-8Neu5Aca2-3)Galb1-4GIcb-Sp0 & 22 & 5 & 23 \\
\hline
\end{tabular}




\begin{tabular}{|c|c|c|c|c|}
\hline 66 & Fuca1-2Galb1-3GlcNAcb1-3Galb1-4Glcb-Sp8 & 22 & 10 & 43 \\
\hline 89 & GlcNAcb1-3Galb1-3GalNAca-Sp8 & 22 & 8 & 37 \\
\hline 360 & KDNa2-3Galb1-3GalNAca-Sp14 & 22 & 16 & 75 \\
\hline 581 & $\begin{array}{l}\text { GlcNAcb1-3Galb1-4GIcNAcb1-3Galb1-4GIcNAcb1-3Galb1-4GlcNAcb1-3Galb1-4GIcNAcb1-2Mana1-6(GlcNAcb1- } \\
\text { 3Galb1-4GIcNAcb1-3Galb1-4GIcNAcb1-3Galb1-4GIcNAcb1-3Galb1-4GIcNAcb1-2Mana1-3)Manb1-4GIcNAcb1- } \\
\text { 4(Fuca1-6)GIcNAcb-Sp19 }\end{array}$ & 22 & 12 & 53 \\
\hline 149 & Galb1-3GIcNAcb1-3Galb1-4GIcb-Sp10 & 22 & 9 & 40 \\
\hline 305 & Galb1-4GIcNAca1-6Galb1-4GIcNAcb-Sp0 & 22 & 10 & 47 \\
\hline 202 & GlcAa-Sp8 & 22 & 7 & 33 \\
\hline 70 & Fuca1-2Galb1-4(Fuca1-3)GIcNAcb1-3Galb1-4(Fuca1-3)GIcNAcb-Sp0 & 22 & 14 & 66 \\
\hline 341 & GlcNAca1-4Galb1-3GlcNAcb-Sp0 & 22 & 11 & 49 \\
\hline 129 & Galb1-3(Fuca1-4)GlcNAc-Sp0 & 22 & 10 & 44 \\
\hline 478 & Neu5Aca2-6Galb1-4GIcNAcb1-6(Galb1-3GlcNAcb1-3)Galb1-4GIcb-Sp21 & 22 & 12 & 54 \\
\hline 90 & GalNAca1-3(Fuca1-2)Galb-Sp8 & 22 & 10 & 48 \\
\hline 56 & $\begin{array}{l}\text { Neu5Aca2-6Galb1-4GIcNAcb1-2Mana1-6(Neu5Aca2-6Galb1-4GIcNAcb1-2Mana1-3)Manb1-4GIcNAcb1- } \\
\text { 4GIcNAcb-Sp13 }\end{array}$ & 22 & 9 & 43 \\
\hline 204 & GlcAb1-3Galb-Sp8 & 22 & 6 & 30 \\
\hline 139 & Neu5Aca2-6(Galb1-3)GIcNAcb1-4Galb1-4Glcb-Sp10 & 22 & 6 & 29 \\
\hline 144 & Galb1-3GalNAcb1-3Gala1-4Galb1-4Glcb-Sp0 & 22 & 14 & 66 \\
\hline 415 & GalNAca1-3(Fuca1-2)Galb1-4GIcNAcb1-3GalNAca-Sp14 & 22 & 10 & 49 \\
\hline 238 & Neu5Aca2-3GalNAcb1-4GIcNAcb-Sp0 & 22 & 14 & 64 \\
\hline 88 & GalNAca1-3(Fuca1-2)Galb1-4Glcb-Sp0 & 22 & 15 & 69 \\
\hline 83 & GalNAca1-3(Fuca1-2)Galb1-3GIcNAcb-Sp0 & 21 & 11 & 53 \\
\hline 12 & Galb-Sp8 & 21 & 9 & 42 \\
\hline 543 & $\begin{array}{l}\text { Galb1-4GIcNAcb1-3Galb1-4GIcNAcb1-2Mana1-6(Galb1-4GIcNAcb1-3Galb1-4GIcNAcb1-2Mana1-3)Manb1- } \\
\text { 4GlcNAcb1-4GIcNAcb-Sp24 }\end{array}$ & 21 & 11 & 50 \\
\hline 267 & Neu5Aca2-6GalNAcb1-4GIcNAcb-Sp0 & 21 & 12 & 58 \\
\hline 123 & Gala1-4Galb1-4Glcb-Sp0 & 21 & 6 & 27 \\
\hline 580 & $\begin{array}{l}\text { Galb1-4GIcNAcb1-3Galb1-4GIcNAcb1-3Galb1-4GIcNAcb1-3Galb1-4GIcNAcb1-2Mana1-6(Galb1-4GlcNAcb1- } \\
\text { 3Galb1-4GIcNAcb1-3Galb1-4GIcNAcb1-3Galb1-4GIcNAcb1-2Mana1-3)Manb1-4GlcNAcb1-4(Fuca1-6)GlcNAcb- } \\
\text { Sp24 }\end{array}$ & 21 & 5 & 24 \\
\hline 584 & $\begin{array}{l}\text { GlcNAcb1-3Galb1-4GlcNAcb1-3Galb1-4GlcNAcb1-6(GlcNAcb1-3Galb1-4GlcNAcb1-3Galb1-4GlcNAb1-2)Mana1- } \\
\text { 6(GlcNAcb1-3Galb1-4GlcNAcb1-3Galb1-4GlcNAcb1-2Mana1-3)Manb1-4GlcNAcb1-4(Fuca1-6)GIcNAcb-Sp24 }\end{array}$ & 21 & 6 & 31 \\
\hline 84 & GalNAca1-3(Fuca1-2)Galb1-4(Fuca1-3)GlcNAcb-Sp0 & 21 & 6 & 29 \\
\hline 349 & Neu5Aca2-6Galb1-4GIcNAcb1-2Mana1-3Manb1-4GIcNAcb1-4GIcNAc-Sp12 & 21 & 12 & 58 \\
\hline 80 & Fuca1-3GIcNAcb-Sp8 & 21 & 3 & 16 \\
\hline 38 & (3S)Galb-Sp8 & 21 & 6 & 29 \\
\hline 365 & Galb1-4GIcNAcb1-2Mana1-6(Mana1-3)Manb1-4GIcNAcb1-4GIcNAcb-Sp12 & 21 & 15 & 71 \\
\hline 275 & Neu5Aca2-6Galb-Sp8 & 21 & 10 & 49 \\
\hline 457 & Neu5Aca2-6Galb1-4GIcNAcb1-6(Fuca1-2Galb1-3GlcNAcb1-3)Galb1-4Glc-Sp21 & 21 & 11 & 51 \\
\hline 425 & GalNAca1-3(Fuca1-2)Galb1-3GlcNAcb1-3GalNAc-Sp14 & 21 & 14 & 66 \\
\hline 609 & $\begin{array}{l}\text { Neu5Aca2-6Galb1-4GlcNAcb1-3Galb1-4GlcNAcb1-2Mana1-6(Neu5Aca2-6Galb1-4GlcNAcb1-3Galb1- } \\
\text { 4GlcNAcb1-2Mana1-3)Manb1-4GIcNAcb1-4GlcNAcb-Sp12 }\end{array}$ & 21 & 4 & 18 \\
\hline
\end{tabular}




\begin{tabular}{|c|c|c|c|c|}
\hline 430 & Galb1-4GlcNAcb1-6(Fuca1-2Galb1-3GlcNAcb1-3)Galb1-4Glc-Sp21 & 21 & 7 & 36 \\
\hline 368 & Neu5Aca2-6GlcNAcb1-4GIcNAcb1-4GIcNAc-Sp21 & 21 & 17 & 82 \\
\hline 166 & Galb1-4GIcNAcb1-3Galb1-4GIcb-Sp8 & 20 & 13 & 63 \\
\hline 93 & GalNAca1-3Galb-Sp8 & 20 & 12 & 58 \\
\hline 299 & (6P)Glcb-Sp10 & 20 & 7 & 35 \\
\hline 505 & (3S)GalNAcb1-4(Fuca1-3)GlcNAcb-Sp8 & 20 & 7 & 36 \\
\hline 254 & Neu5Aca2-3Galb1-4(Fuca1-3)GIcNAcb1-3Galb1-4(Fuca1-3)GlcNAcb1-3Galb1-4(Fuca1-3)GlcNAcb-Sp0 & 20 & 12 & 60 \\
\hline 59 & Fuca1-2Galb1-3GalNAcb1-3Gala-Sp9 & 20 & 11 & 57 \\
\hline 477 & Galb1-3GIcNAcb1-2Mana1-6(GIcNAcb1-4)(Galb1-3GIcNAcb1-2Mana1-3)Manb1-4GIcNAcb1-4GIcNAcb-Sp21 & 20 & 2 & 9 \\
\hline 465 & $\begin{array}{l}\text { Neu5Aca2-6Galb1-4GlcNAcb1-4Mana1-6(GlcNAcb1-4)(Neu5Aca2-6Galb1-4GlcNAcb1-4(Neu5Aca2-6Galb1- } \\
\text { 4GlcNAcb1-2)Mana1-3)Manb1-4GlcNAcb1-4GlcNAcb-Sp21 }\end{array}$ & 20 & 13 & 66 \\
\hline 270 & Neu5Aca2-6Galb1-4GIcNAcb-Sp8 & 20 & 15 & 74 \\
\hline 152 & Galb1-4(Fuca1-3)GlcNAcb-Sp0 & 20 & 2 & 10 \\
\hline 223 & Fuca1-2(6S)Galb1-4(6S)Glcb-Sp0 & 20 & 10 & 50 \\
\hline 602 & Neu5Aca2-6Galb1-4GIcNAcb1-3Galb1-4GIcNAcb1-6(Galb1-3)GalNAca-Sp14 & 20 & 7 & 35 \\
\hline 443 & GalNAcb1-6GalNAcb-Sp8 & 20 & 5 & 28 \\
\hline 455 & $\begin{array}{l}\text { GalNAca1-3(Fuca1-2)Galb1-4GIcNAcb1-2Mana1-6(GalNAca1-3(Fuca1-2)Galb1-4GlcNAcb1-2Mana1-3)Manb1- } \\
\text { 4GlcNAcb1-4(Fuca1-6)GlcNAcb-Sp22 }\end{array}$ & 20 & 10 & 51 \\
\hline 397 & $\begin{array}{l}\text { Neu5Aca2-3Galb1-3GIcNAcb1-2Mana1-6(Neu5Aca2-3Galb1-3GlcNAcb1-2Mana1-3)Manb1-4GIcNAcb1- } \\
\text { 4GIcNAc-Sp19 }\end{array}$ & 20 & 11 & 56 \\
\hline 499 & Gala1-3(Fuca1-2)Galb1-4GIcNAcb1-6GalNAca-Sp14 & 20 & 14 & 71 \\
\hline 184 & GIcNAcb1-3Galb1-4GlcNAcb-Sp8 & 20 & 15 & 76 \\
\hline 264 & Neu5Aca2-3Galb1-4GIcb-Sp0 & 20 & 7 & 37 \\
\hline 23 & $6 \mathrm{~S}(3 \mathrm{~S}) \mathrm{Galb1}-4 \mathrm{GlcNAcb}-\mathrm{Sp} 0$ & 20 & 7 & 35 \\
\hline 516 & (3S)GalNAcb1-4GIcNAc-Sp8 & 20 & 7 & 38 \\
\hline 215 & Mana1-2Mana1-2Mana1-6(Mana1-3)Mana-Sp9 & 19 & 9 & 45 \\
\hline 588 & $\begin{array}{l}\text { GlcNAcb1-3Galb1-4GIcNAcb1-3Galb1-4GlcNAcb1-3Galb1-4GIcNAcb1-3Galb1-4GlcNAcb1-6(GIcNAcb1-3Galb1- } \\
\text { 4GlcNAcb1-3Galb1-4GIcNAcb1-3Galb1-4GlcNAcb1-3Galb1-4GlcNAb1-2)Mana1-6(GlcNAcb1-3Galb1- } \\
\text { 4GlcNAcb1-3Galb1-4GlcNAcb1-3Galb1-4GlcNAcb1-3Galb1-4GlcNAcb1-2Mana1-3)Manb1-4GlcNAcb1-4(Fuca1- } \\
\text { 6)GlcNAcb-Sp24 }\end{array}$ & 19 & 6 & 29 \\
\hline 146 & Galb1-3GalNAcb1-4Galb1-4Glcb-Sp8 & 19 & 7 & 35 \\
\hline 391 & Fuca1-2Galb1-3GalNAca1-3(Fuca1-2)Galb1-4GIcNAcb-Sp0 & 19 & 5 & 27 \\
\hline 387 & Galb1-3GlcNAcb1-3Galb1-4(Fuca1-3)GIcNAcb1-6(Galb1-3GIcNAcb1-3)Galb1-4GIc-Sp21 & 19 & 12 & 61 \\
\hline 155 & Galb1-4(Fuca1-3)GlcNAcb1-3Galb1-4(Fuca1-3)GlcNAcb1-3Galb1-4(Fuca1-3)GIcNAcb-Sp0 & 19 & 5 & 29 \\
\hline 294 & Galb1-4GIcNAcb1-3Galb1-3GIcNAcb-Sp0 & 19 & 14 & 75 \\
\hline 492 & Neu5Aca2-3Galb1-3GIcNAcb1-6GalNAca-Sp14 & 19 & 8 & 41 \\
\hline 182 & GlcNAcb1-3Galb-Sp8 & 19 & 15 & 78 \\
\hline 177 & GlcNAcb1-6(GlcNAcb1-3)GalNAca-Sp8 & 19 & 5 & 25 \\
\hline 428 & $\begin{array}{l}\text { Gala1-3(Fuca1-2)Galb1-4GlcNAcb1-2Mana1-6(Gala1-3(Fuca1-2)Galb1-4GIcNAcb1-2Mana1-3)Manb1- } \\
\text { 4GlcNAcb1-4(Fuca1-6)GlcNAcb-Sp22 }\end{array}$ & 19 & 11 & 57 \\
\hline 394 & GalNAca1-3(Fuca1-2)Galb1-3GalNAca1-3(Fuca1-2)Galb1-4GIcNAcb-Sp0 & 19 & 6 & 32 \\
\hline 52 & GlcNAcb1-2Mana1-6(GlcNAcb1-2Mana1-3)Manb1-4GIcNAcb1-4GIcNAcb-Sp12 & 19 & 7 & 35 \\
\hline 95 & GalNAcb1-3GalNAca-Sp8 & 19 & 16 & 86 \\
\hline
\end{tabular}




\begin{tabular}{|c|c|c|c|c|}
\hline 9 & Neu5Aca-Sp8 & 19 & 9 & 49 \\
\hline 308 & GlcAb1-3GlcNAcb-Sp8 & 19 & 16 & 86 \\
\hline 61 & Fuca1-2Galb1-3(Fuca1-4)GIcNAcb-Sp8 & 19 & 7 & 36 \\
\hline 491 & Galb1-3(Fuca1-4)GlcNAcb1-6GalNAca-Sp14 & 19 & 7 & 40 \\
\hline 148 & Galb1-3GIcNAcb1-3Galb1-4GIcNAcb-Sp0 & 19 & 17 & 89 \\
\hline 335 & GalNAcb1-3Gala1-4Galb1-4GIcNAcb1-3Galb1-4GIcb-Sp0 & 19 & 18 & 97 \\
\hline 92 & GalNAca1-3GalNAcb-Sp8 & 19 & 23 & 125 \\
\hline 410 & Gala1-3(Fuca1-2)Galb1-4(Fuca1-3)Glcb-Sp21 & 18 & 18 & 97 \\
\hline 489 & Galb1-3GIcNAcb1-6GalNAca-Sp14 & 18 & 8 & 44 \\
\hline 208 & Mana1-2Mana1-2Mana1-3Mana-Sp9 & 18 & 4 & 23 \\
\hline 244 & Neu5Aca2-6(Neu5Aca2-3Galb1-3)GalNAca-Sp8 & 18 & 7 & 40 \\
\hline 452 & GalNAca1-3(Fuca1-2)Galb1-4GIcNAcb1-6(GalNAca1-3(Fuca1-2)Galb1-4GlcNAcb1-3)GalNAc-Sp14 & 18 & 7 & 39 \\
\hline 191 & GlcNAcb1-4GlcNAcb1-4GIcNAcb1-4GlcNAcb1-4GlcNAcb1-Sp8 & 18 & 11 & 57 \\
\hline 122 & Gala1-4Galb1-4GlcNAcb-Sp8 & 18 & 2 & 13 \\
\hline 22 & $6 \mathrm{~S}(3 \mathrm{~S})$ Galb1-4(6S)GIcNAcb-Sp0 & 18 & 19 & 105 \\
\hline 142 & Galb1-3GalNAca-Sp16 & 18 & 8 & 42 \\
\hline 550 & $\begin{array}{l}\text { GlcNAcb1-3Galb1-4GlcNAcb1-3Galb1-4GIcNAcb1-3Galb1-4GlcNAcb1-2Mana1-6(GlcNAcb1-3Galb1-4GIcNAcb1- } \\
\text { 3Galb1-4GlcNAcb1-3Galb1-4GIcNAcb1-2Mana1-3)Manb1-4GIcNAcb1-4GIcNAcb-Sp25 }\end{array}$ & 18 & 11 & 61 \\
\hline 424 & Gala1-3(Fuca1-2)Galb1-3GIcNAcb1-3GalNAc-Sp14 & 18 & 9 & 50 \\
\hline 79 & Fuca1-2Galb-Sp8 & 18 & 12 & 67 \\
\hline 141 & Galb1-3GalNAca-Sp14 & 18 & 13 & 74 \\
\hline 13 & Glcb-Sp8 & 18 & 12 & 67 \\
\hline 103 & Gala1-3(Fuca1-2)Galb1-3GlcNAcb-Sp8 & 18 & 3 & 16 \\
\hline 127 & Galb1-3(Fuca1-4)GlcNAcb1-3Galb1-4(Fuca1-3)GIcNAcb-Sp0 & 18 & 8 & 43 \\
\hline 404 & Gala1-4Galb1-3GlcNAcb1-2Mana1-6(Gala1-4Galb1-3GlcNAcb1-2Mana1-3)Manb1-4GIcNAcb1-4GlcNAcb-Sp19 & 18 & 1 & 8 \\
\hline 198 & Glca1-6Glca1-6Glcb-Sp8 & 18 & 8 & 43 \\
\hline 185 & GIcNAcb1-3Galb1-4GlcNAcb1-3Galb1-4GlcNAcb-Sp0 & 18 & 13 & 70 \\
\hline 332 & Neu5Aca2-3Galb1-3(Fuca1-4)GIcNAcb1-3Galb1-3(Fuca1-4)GIcNAcb-Sp0 & 18 & 7 & 39 \\
\hline 74 & Fuca1-2Galb1-4GIcNAcb1-3Galb1-4GIcNAcb-Sp0 & 18 & 4 & 24 \\
\hline 524 & GalNAca1-3(Fuca1-2)Galb1-4 GlcNAcb1-2Mana-Sp0 & 18 & 15 & 84 \\
\hline 488 & Neu5Aca2-6Galb1-4GIcNAcb1-6(Fuca1-2Galb1-4(Fuca1-3)GIcNAcb1-3)Galb1-4GIc-Sp21 & 18 & 13 & 73 \\
\hline 563 & GalNAcb1-3GlcNAcb-Sp0 & 18 & 9 & 50 \\
\hline 199 & Glcb1-4Glcb-Sp8 & 18 & 9 & 51 \\
\hline 504 & GalNAcb1-4(Fuca1-3)(6S)GlcNAcb-Sp8 & 17 & 6 & 34 \\
\hline 375 & $\begin{array}{l}\text { Gala1-3(Fuca1-2)Galb1-3GlcNAcb1-2Mana1-6(Gala1-3(Fuca1-2)Galb1-3GlcNAcb1-2Mana1-3)Manb1- } \\
\text { 4GlcNAcb1-4GlcNAcb-Sp20 }\end{array}$ & 17 & 6 & 36 \\
\hline 157 & Galb1-4(6S)Glcb-Sp8 & 17 & 2 & 9 \\
\hline 481 & Neu5Aca2-6Galb1-4GIcNAcb1-6GalNAca-Sp14 & 17 & 6 & 33 \\
\hline
\end{tabular}




\begin{tabular}{|c|c|c|c|c|}
\hline 114 & Gala1-3Galb1-4(Fuca1-3)GlcNAcb-Sp8 & 17 & 8 & 45 \\
\hline 348 & Neu5Aca2-6Galb1-4GIcNAcb1-2Mana1-6Manb1-4GlcNAcb1-4GlcNAc-Sp12 & 17 & 10 & 55 \\
\hline 109 & Gala1-3(Fuca1-2)Galb-Sp18 & 17 & 12 & 71 \\
\hline 577 & $\begin{array}{l}\text { GlcNAcb1-3Galb1-4GIcNAcb1-3Galb1-4GIcNAcb1-2Mana1-6(GlcNAcb1-3Galb1-4GIcNAcb1-3Galb1-4GIcNAcb1- } \\
\text { 2Mana1-3)Manb1-4GIcNAcb1-4(Fuca1-6)GIcNAcb-Sp24 }\end{array}$ & 17 & 8 & 46 \\
\hline 137 & Neu5Aca2-6(Galb1-3)GalNAca-Sp14 & 17 & 6 & 34 \\
\hline 33 & (3S)Galb1-4(Fuca1-3)GlcNAc-Sp8 & 17 & 4 & 26 \\
\hline 72 & Fuca1-2Galb1-4(Fuca1-3)GlcNAcb-Sp0 & 17 & 19 & 108 \\
\hline 104 & Gala1-3(Fuca1-2)Galb1-4(Fuca1-3)GIcNAcb-Sp0 & 17 & 13 & 73 \\
\hline 172 & Galb1-4GIcb-Sp0 & 17 & 9 & 53 \\
\hline 180 & GIcNAcb1-3GalNAca-Sp8 & 17 & 15 & 86 \\
\hline 134 & GlcNAcb1-6(Galb1-3)GalNAca-Sp8 & 17 & 3 & 17 \\
\hline 527 & Neu5Aca2-3Galb1-3GlcNAcb1-2Mana-Sp0 & 17 & 7 & 43 \\
\hline 21 & GlcNAcb1-6(GlcNAcb1-4)(GlcNAcb1-3)GlcNAc-Sp8 & 17 & 8 & 48 \\
\hline 367 & Neu5Aca2-6GIcNAcb1-4GIcNAc-Sp21 & 17 & 8 & 45 \\
\hline 304 & GlcNAcb1-6(Galb1-4GlcNAcb1-3)Galb1-4GlcNAc-Sp0 & 17 & 6 & 33 \\
\hline 444 & (6S)Galb1-3GlcNAcb-Sp0 & 17 & 7 & 39 \\
\hline 249 & Fuca1-2(6S)Galb1-4Glcb-Sp0 & 17 & 9 & 55 \\
\hline 353 & GlcNAcb1-2Mana1-6(GlcNAcb1-2Mana1-3)Manb1-4GlcNAcb1-4(Fuca1-6)GIcNAcb-Sp22 & 17 & 8 & 48 \\
\hline 32 & (3S)Galb1-4(Fuca1-3)GlcNAc-Sp0 & 17 & 11 & 66 \\
\hline 298 & (6S)Galb1-4(6S)GIcNAcb-Sp0 & 17 & 18 & 106 \\
\hline 288 & Neu5Gca-Sp8 & 17 & 12 & 74 \\
\hline 271 & Neu5Aca2-6Galb1-4GIcNAcb1-3Galb1-4(Fuca1-3)GlcNAcb1-3Galb1-4(Fuca1-3)GlcNAcb-Sp0 & 17 & 7 & 41 \\
\hline 518 & Galb1-4(6P)GlcNAcb-Sp0 & 17 & 14 & 86 \\
\hline 309 & Neu5Aca2-6Galb1-4GIcNAcb1-2Mana1-6(GlcNAcb1-2Mana1-3)Manb1-4GlcNAcb1-4GlcNAcb-Sp12 & 16 & 9 & 56 \\
\hline 230 & Neu5Aca2-8Neu5Aca2-8Neu5Aca-Sp8 & 16 & 5 & 31 \\
\hline 502 & Fuca1-2(6S)Galb1-3(6S)GlcNAcb-Sp0 & 16 & 4 & 23 \\
\hline 181 & GlcNAcb1-3GalNAca-Sp14 & 16 & 7 & 44 \\
\hline 539 & Galb1-3GalNAcb1-3Gal-Sp21 & 16 & 4 & 23 \\
\hline 471 & Glca1-4Glca1-4Glca1-4Glcb-Sp10 & 16 & 4 & 24 \\
\hline 475 & $\begin{array}{l}\text { Neu5Aca2-3Galb1-3GIcNAcb1-6(Neu5Aca2-3Galb1-4GIcNAcb1-2)Mana1-6(Neu5Aca2-3Galb1-3GIcNAcb1- } \\
\text { 2Mana1-3)Manb1-4GIcNAcb1-4GIcNAcb-Sp19 }\end{array}$ & 16 & 9 & 53 \\
\hline 513 & (6S)GalNAcb1-4GIcNAc-Sp8 & 16 & 8 & 48 \\
\hline 234 & GalNAcb1-4(Neu5Aca2-3)Galb1-4Glcb-Sp0 & 16 & 10 & 62 \\
\hline 611 & Galb1-3GalNAcb1-4(Neu5Aca2-8Neu5Aca2-8Neu5Aca2-3)Galb1-4GIcb-Sp21 & 16 & 7 & 40 \\
\hline 44 & (6S)Galb1-4GlcNAcb-Sp8 & 16 & 10 & 60 \\
\hline 431 & Fuca1-3GlcNAcb1-6(Galb1-4GlcNAcb1-3)Galb1-4Glc-Sp21 & 16 & 11 & 68 \\
\hline 281 & Neu5Gca2-3Galb1-3(Fuca1-4)GlcNAcb-Sp0 & 16 & 7 & 43 \\
\hline 358 & KDNa2-6Galb1-4GIcNAc-Sp0 & 16 & 10 & 64 \\
\hline 434 & GlcNAcb1-6(GlcNAcb1-2)Mana1-6(GlcNAcb1-4)(GlcNAcb1-2Mana1-3)Manb1-4GIcNAcb1-4GIcNAc-Sp21 & 16 & 17 & 108 \\
\hline 221 & Fuca1-2(6S)Galb1-4GIcNAcb-Sp0 & 16 & 8 & 53 \\
\hline
\end{tabular}




\begin{tabular}{|c|c|c|c|c|}
\hline 195 & GIcNAcb1-6Galb1-4GIcNAcb-Sp8 & 16 & 15 & 91 \\
\hline 205 & GlcAb1-6Galb-Sp8 & 16 & 6 & 35 \\
\hline 171 & Galb1-4GlcNAcb-Sp23 & 16 & 14 & 89 \\
\hline 339 & GlcNAca1-4Galb1-4GIcNAcb1-3Galb1-4GlcNAcb1-3Galb1-4GIcNAcb-Sp0 & 16 & 12 & 75 \\
\hline 1 & Gala-Sp8 & 16 & 15 & 95 \\
\hline 445 & (6S)Galb1-3(6S)GIcNAc-Sp0 & 16 & 6 & 38 \\
\hline 138 & Neu5Acb2-6(Galb1-3)GalNAca-Sp8 & 16 & 14 & 87 \\
\hline 512 & $(6 S)(4 S) G a l N A c b 1-4 G l c N A c-S p 8$ & 16 & 7 & 46 \\
\hline 479 & Neu5Aca2-3Galb1-4GlcNAcb1-2Mana-Sp0 & 16 & 1 & 9 \\
\hline 78 & Fuca1-2Galb1-4GIcb-Sp0 & 16 & 17 & 105 \\
\hline 306 & Galb1-4GIcNAcb1-6Galb1-4GIcNAcb-Sp0 & 16 & 6 & 36 \\
\hline 433 & GlcNAcb1-2Mana1-6(GIcNAcb1-4)(GIcNAcb1-4(GlcNAcb1-2)Mana1-3)Manb1-4GlcNAcb1-4GIcNAc-Sp21 & 16 & 12 & 80 \\
\hline 467 & $\begin{array}{l}\text { Neu5Aca2-6Galb1-4GlcNAcb1-6(Neu5Aca2-6Galb1-4GlcNAcb1-2)Mana1-6(GlcNAcb1-4)(Neu5Aca2-6Galb1- } \\
\text { 4GlcNAcb1-4(Neu5Aca2-6Galb1-4GlcNAcb1-2)Mana1-3)Manb1-4GlcNAcb1-4GlcNAcb-Sp21 }\end{array}$ & 15 & 3 & 18 \\
\hline 213 & Mana1-2Mana1-6(Mana1-2Mana1-3)Mana1-6(Mana1-2Mana1-2Mana1-3)Manb1-4GIcNAcb1-4GIcNAcb-Sp12 & 15 & 4 & 26 \\
\hline 135 & GlcNAcb1-6(Galb1-3)GalNAca-Sp14 & 15 & 16 & 104 \\
\hline 566 & $\begin{array}{l}\text { Galb1-4GIcNAcb1-3Galb1-4GIcNAcb1-3Galb1-4GIcNAcb1-3Galb1-4GlcNAcb1-3Galb1-4GIcNAcb1-2Mana1- } \\
\text { 6(Galb1-4GlcNAcb1-3Galb1-4GIcNAcb1-3Galb1-4GIcNAcb1-3Galb1-4GlcNAcb1-3Galb1-4GIcNAcb1-2Mana1- } \\
\text { 3)Manb1-4GIcNAcb1-4GIcNAcb-Sp25 }\end{array}$ & 15 & 7 & 44 \\
\hline 540 & $\begin{array}{l}\text { GlcNAcb1-3Galb1-4GlcNAcb1-2Mana1-6(GlcNAcb1-3Galb1-4GlcNAcb1-2Mana1-3)Manb1-4GlcNAcb1- } \\
\text { 4GlcNAcb-Sp12 }\end{array}$ & 15 & 5 & 33 \\
\hline 229 & GalNAcb1-4(Neu5Aca2-8Neu5Aca2-3)Galb1-4Glcb-Sp0 & 15 & 18 & 121 \\
\hline 369 & Galb1-4(Fuca1-3)GlcNAcb1-6(Fuca1-2Galb1-4GIcNAcb1-3)Galb1-4GIc-Sp21 & 15 & 1 & 9 \\
\hline 257 & Neu5Aca2-3Galb1-4(Fuca1-3)GIcNAcb1-3Galb-Sp8 & 15 & 23 & 157 \\
\hline 263 & Fuca1-2Galb1-4(6S)GIcb-Sp0 & 15 & 6 & 40 \\
\hline 167 & Galb1-4GIcNAcb1-6(Galb1-3)GalNAca-Sp8 & 15 & 5 & 33 \\
\hline 161 & Galb1-4GIcNAcb1-3GalNAc-Sp14 & 15 & 9 & 63 \\
\hline 14 & Manb-Sp8 & 15 & 4 & 27 \\
\hline 159 & Galb1-4GalNAcb1-3(Fuca1-2)Galb1-4GlcNAcb-Sp8 & 15 & 9 & 58 \\
\hline 325 & Galb1-3GIcNAcb1-2Mana1-6(Galb1-3GIcNAcb1-2Mana1-3)Manb1-4GIcNAcb1-4GIcNAcb-Sp19 & 15 & 7 & 49 \\
\hline 105 & Gala1-3(Fuca1-2)Galb1-4(Fuca1-3)GlcNAcb-Sp8 & 15 & 14 & 97 \\
\hline 297 & $4 \mathrm{~S}(3 \mathrm{~S}) \mathrm{Galb1}-4 \mathrm{GlcNAcb}-\mathrm{Sp} 0$ & 15 & 6 & 44 \\
\hline 487 & $\begin{array}{l}\text { Neu5Aca2-3Galb1-3GIcNAcb1-2Mana1-6(GIcNAcb1-4)(Neu5Aca2-3Galb1-3GlcNAcb1-2Mana1-3)Manb1- } \\
\text { 4GlcNAcb1-4GlcNAc-Sp21 }\end{array}$ & 15 & 7 & 51 \\
\hline 17 & GlcNAcb-Sp8 & 15 & 7 & 50 \\
\hline 282 & Neu5Gca2-3Galb1-3GIcNAcb-Sp0 & 15 & 4 & 30 \\
\hline 222 & Fuca1-2Galb1-4(6S)GIcNAcb-Sp8 & 14 & 4 & 30 \\
\hline 507 & GalNAca1-3(Fuca1-2)Galb1-3GIcNAcb1-6GalNAca-Sp14 & 14 & 10 & 69 \\
\hline 250 & Neu5Aca2-3Galb1-3GIcNAcb-Sp0 & 14 & 6 & 39 \\
\hline
\end{tabular}




\begin{tabular}{|c|c|c|c|c|}
\hline 313 & MurNAcb1-4GIcNAcb-Sp10 & 14 & 9 & 59 \\
\hline 441 & Galb1-6Galb-Sp10 & 14 & 6 & 44 \\
\hline 316 & Mana1-2Mana1-6(Mana1-3)Mana1-6(Mana1-2Mana1-2Mana1-3)Mana-Sp9 & 14 & 5 & 34 \\
\hline 211 & Mana1-6(Mana1-2Mana1-3)Mana1-6(Mana1-2Mana1-3)Manb1-4GIcNAcb1-4GIcNAcb-Sp12 & 14 & 6 & 40 \\
\hline 336 & GalNAca1-3(Fuca1-2)Galb1-4GIcNAcb1-3Galb1-4GIcNAcb-Sp0 & 14 & 6 & 40 \\
\hline 68 & Fuca1-2Galb1-3GlcNAcb-Sp0 & 14 & 12 & 86 \\
\hline 382 & Galb1-3GalNAca1-3(Fuca1-2)Galb1-4GIcNAc-Sp0 & 14 & 5 & 33 \\
\hline 160 & Galb1-4GIcNAcb1-3GalNAca-Sp8 & 14 & 10 & 69 \\
\hline 48 & $\mathrm{Neu} 5,9 \mathrm{Ac}_{2} \mathrm{a}-\mathrm{Sp} 8$ & 14 & 2 & 17 \\
\hline 314 & Mana1-6Manb-Sp10 & 14 & 8 & 60 \\
\hline 564 & GalNAcb1-4GIcNAcb1-3GalNAcb1-4GIcNAcb-Sp0 & 14 & 10 & 69 \\
\hline 143 & Galb1-3GalNAcb-Sp8 & 14 & 6 & 41 \\
\hline 18 & $\mathrm{GlcN}(\mathrm{Gc}) \mathrm{b}-\mathrm{Sp} 8$ & 14 & 5 & 39 \\
\hline 386 & Galb1-4(Fuca1-3)GIcNAcb1-6(Fuca1-4(Fuca1-2Galb1-3)GlcNAcb1-3)Galb1-4Glc-Sp21 & 14 & 12 & 84 \\
\hline 62 & Fuca1-2Galb1-3GalNAca-Sp8 & 14 & 12 & 90 \\
\hline 352 & Mana1-6(Galb1-4GlcNAcb1-2Mana1-3)Manb1-4GIcNAcb1-4GlcNAcb-Sp12 & 14 & 7 & 48 \\
\hline 322 & Neu5Aca2-8Neu5Acb-Sp17 & 14 & 8 & 58 \\
\hline 162 & Galb1-4GIcNAcb1-3Galb1-4(Fuca1-3)GIcNAcb1-3Galb1-4(Fuca1-3)GlcNAcb-Sp0 & 14 & 16 & 116 \\
\hline 100 & GalNAcb1-4GIcNAcb-Sp8 & 14 & 16 & 118 \\
\hline 330 & Neu5,9Ac2a2-3Galb1-3GlcNAcb-Sp0 & 13 & 15 & 110 \\
\hline 175 & GIcNAca1-6Galb1-4GIcNAcb-Sp8 & 13 & 13 & 97 \\
\hline 347 & Mana1-6(Neu5Aca2-6Galb1-4GIcNAcb1-2Mana1-3)Manb1-4GIcNAcb1-4GIcNAc-Sp12 & 13 & 14 & 101 \\
\hline 311 & GlcNAcb1-4GIcNAcb-Sp10 & 13 & 11 & 85 \\
\hline 600 & Galb1-4GIcNAcb1-3Galb1-3GalNAca-Sp14 & 13 & 8 & 56 \\
\hline 28 & (3S)Galb1-3(Fuca1-4)GlcNAcb-Sp8 & 13 & 9 & 64 \\
\hline 482 & Neu5Aca2-6Galb1-4 GlcNAcb1-6(Neu5Aca2-6Galb1-4GlcNAcb1-3)GalNAca-Sp14 & 13 & 5 & 39 \\
\hline 255 & Neu5Aca2-3Galb1-4(Fuca1-3)GlcNAcb-Sp0 & 13 & 6 & 44 \\
\hline 400 & Neu5Aca2-3Galb1-3GIcNAcb1-3GalNAca-Sp14 & 13 & 11 & 87 \\
\hline 547 & $\begin{array}{l}\text { GIcNAcb1-3Galb1-4GlcNAcb1-3Galb1-4GIcNAcb1-2Mana1-6(GlcNAcb1-3Galb1-4GlcNAcb1-3Galb1-4GIcNAcb1- } \\
\text { 2Mana1-3)Manb1-4GIcNAcb1-4GlcNAcb-Sp25 }\end{array}$ & 13 & 10 & 74 \\
\hline 521 & Neu5Aca2-6Galb1-4GIcNAcb1-2Man-Sp0 & 13 & 11 & 85 \\
\hline 402 & Galb1-4(Fuca1-3)GIcNAcb1-3GalNAca-Sp14 & 13 & 10 & 77 \\
\hline 51 & Mana1-6(Mana1-3)Manb1-4GlcNAcb1-4GlcNAcb-Sp13 & 13 & 9 & 66 \\
\hline
\end{tabular}




\begin{tabular}{|c|c|c|c|c|}
\hline 537 & GalNAca1-3(Fuca1-2)Galb1-3GalNAcb1-3Gala1-4Galb1-4Glc-Sp21 & 13 & 13 & 99 \\
\hline 389 & GlcNAcb1-2Mana1-6(GlcNAcb1-4(GlcNAcb1-2)Mana1-3)Manb1-4GlcNAcb1-4GIcNAc-Sp21 & 13 & 13 & 97 \\
\hline 317 & Mana1-2Mana1-6(Mana1-2Mana1-3)Mana1-6(Mana1-2Mana1-2Mana1-3)Mana-Sp9 & 13 & 5 & 36 \\
\hline 54 & Galb1-4GlcNAcb1-2Mana1-6(Galb1-4GlcNAcb1-2Mana1-3)Manb1-4GlcNAcb1-4GlcNAcb-Sp12 & 13 & 18 & 136 \\
\hline 592 & Galb1-4GIcNAcb1-3Galb1-4GIcNAcb1-6(Galb1-4GIcNAcb1-3Galb1-4GIcNAcb1-3)GalNAca-Sp14 & 13 & 9 & 73 \\
\hline 158 & Galb1-4GalNAca1-3(Fuca1-2)Galb1-4GlcNAcb-Sp8 & 13 & 3 & 26 \\
\hline 242 & Neu5Aca2-3Galb1-4(Neu5Aca2-3Galb1-3)GlcNAcb-Sp8 & 13 & 10 & 78 \\
\hline 20 & Galb1-4GlcNAcb1-6(Galb1-4GlcNAcb1-3)GalNAc-Sp14 & 13 & 7 & 51 \\
\hline 458 & $\begin{array}{l}\text { GalNAca1-3(Fuca1-2)Galb1-3GIcNAcb1-2Mana1-6(GalNAca1-3(Fuca1-2)Galb1-3GIcNAcb1-2Mana1-3)Manb1- } \\
\text { 4GIcNAcb1-4(Fuca1-6)GIcNAcb-Sp22 }\end{array}$ & 13 & 4 & 31 \\
\hline 599 & GIcNAcb1-3Galb1-4GIcNAcb1-3Galb1-4GIcNAcb1-3GalNAca-Sp14 & 13 & 15 & 117 \\
\hline 178 & GIcNAcb1-6(GlcNAcb1-3)GalNAca-Sp14 & 13 & 9 & 67 \\
\hline 427 & $\begin{array}{l}\text { Fuca1-2Galb1-3GIcNAcb1-2Mana1-6(Fuca1-2Galb1-3GIcNAcb1-2Mana1-3)Manb1-4GIcNAcb1-4(Fuca1- } \\
\text { 6)GIcNAcb-Sp22 }\end{array}$ & 13 & 11 & 90 \\
\hline 531 & GlcNAcb1-2 Mana1-6(GlcNAcb1-4)(GlcNAcb1-2Mana1-3)Manb1-4GlcNAcb1-4(Fuca1-6)GlcNAc-Sp21 & 13 & 8 & 65 \\
\hline 289 & Neu5Aca2-3Galb1-4GIcNAcb1-6(Galb1-3)GalNAca-Sp14 & 12 & 5 & 39 \\
\hline 295 & Neu5Aca2-3Galb1-3GlcNAcb1-3Galb1-3GlcNAcb-Sp0 & 12 & 6 & 47 \\
\hline 170 & Galb1-4GlcNAcb-Sp8 & 12 & 5 & 41 \\
\hline 498 & Fuca1-2(6S)Galb1-3GIcNAcb-Sp0 & 12 & 9 & 74 \\
\hline 145 & Galb1-3GalNAcb1-4(Neu5Aca2-3)Galb1-4GIcb-Sp0 & 12 & 5 & 38 \\
\hline 188 & GlcNAcb1-6(GlcNAcb1-4)GalNAca-Sp8 & 12 & 7 & 62 \\
\hline 474 & $\begin{array}{l}\text { Fuca1-2Galb1-3(Fuca1-4)GIcNAcb1-2Mana1-6(Fuca1-2Galb1-3(Fuca1-4)GlcNAcb1-2Mana1-3)Manb1- } \\
\text { 4GlcNAcb1-4(Fuca1-6)GlcNAcb1-4(Fuca1-6)GIcNAcb-Sp19 }\end{array}$ & 12 & 10 & 79 \\
\hline 106 & Gala1-3(Fuca1-2)Galb1-4GIcNAc-Sp0 & 12 & 8 & 64 \\
\hline 163 & Galb1-4GlcNAcb1-3Galb1-4GlcNAcb1-3Galb1-4GlcNAcb-Sp0 & 12 & 8 & 63 \\
\hline 7 & Fuca-Sp9 & 12 & 12 & 104 \\
\hline 390 & Fuca1-2Galb1-3GalNAca1-3(Fuca1-2)Galb1-4Glcb-Sp0 & 12 & 9 & 79 \\
\hline 453 & Neu5Aca2-8Neu5Aca2-3Galb1-3GalNAcb1-4(Neu5Aca2-8Neu5Aca2-3)Galb1-4GIcb-Sp0 & 12 & 11 & 93 \\
\hline 274 & Neu5Aca2-6Galb1-4GIcb-Sp8 & 12 & 4 & 38 \\
\hline 272 & Neu5Aca2-6Galb1-4GIcNAcb1-3Galb1-4GIcNAcb-Sp0 & 12 & 9 & 77 \\
\hline 50 & Mana1-6(Mana1-3)Manb1-4GIcNAcb1-4GIcNAcb-Sp12 & 12 & 5 & 40 \\
\hline 251 & Neu5Aca2-3Galb1-3GIcNAcb-Sp8 & 12 & 7 & 64 \\
\hline 403 & GaINAca1-3GalNAcb1-3Gala1-4Galb1-4GIcNAcb-Sp0 & 12 & 10 & 88 \\
\hline 153 & Galb1-4(Fuca1-3)GlcNAcb-Sp8 & 12 & 6 & 55 \\
\hline 119 & Gala1-3Galb-Sp8 & 11 & 11 & 96 \\
\hline 266 & Neu5Aca2-6GalNAca-Sp8 & 11 & 10 & 88 \\
\hline 194 & GlcNAcb1-6GalNAca-Sp14 & 11 & 6 & 54 \\
\hline 39 & $(6 S)(4 S) G a l b 1-4 G I c N A c b-S p 0$ & 11 & 3 & 24 \\
\hline 567 & GlcNAb1-3Galb1-3GalNAc-Sp14 & 11 & 10 & 89 \\
\hline 422 & GlcNAcb1-2(GlcNAcb1-6)Mana1-6(GlcNAcb1-2Mana1-3)Manb1-4GlcNAcb1-4GlcNAcb-Sp19 & 11 & 10 & 91 \\
\hline
\end{tabular}




\begin{tabular}{|c|c|c|c|c|}
\hline 398 & GIcNAcb1-2Mana1-6(Galb1-4GIcNAcb1-2Mana1-3)Manb1-4GIcNAcb1-4GIcNAc-Sp12 & 11 & 7 & 66 \\
\hline 610 & GIcNAcb1-3Fuca-Sp21 & 11 & 5 & 46 \\
\hline 344 & GIcNAca1-4Galb1-4GIcNAcb1-3Galb1-4GIcNAcb-Sp0 & 11 & 5 & 48 \\
\hline 164 & Galb1-4GIcNAcb1-3Galb1-4GIcNAcb-Sp0 & 11 & 10 & 87 \\
\hline 65 & Fuca1-2Galb1-3GalNAcb1-4(Neu5Aca2-3)Galb1-4Glcb-Sp9 & 11 & 4 & 35 \\
\hline 497 & Galb1-4(Fuca1-3)GIcNAcb1-2Mana-Sp0 & 11 & 6 & 52 \\
\hline 190 & GlcNAcb1-4GlcNAcb1-4GIcNAcb1-4GlcNAcb1-4GIcNAcb1-4GlcNAcb1-Sp8 & 11 & 5 & 42 \\
\hline 173 & Galb1-4Glcb-Sp8 & 11 & 5 & 44 \\
\hline 262 & Neu5Aca2-3Galb1-4GIcNAcb1-3Galb1-4GIcNAcb-Sp0 & 11 & 4 & 41 \\
\hline 112 & Gala1-3GalNAca-Sp16 & 11 & 7 & 65 \\
\hline 3 & Mana-Sp8 & 11 & 9 & 79 \\
\hline 283 & Neu5Gca2-3Galb1-4(Fuca1-3)GIcNAcb-Sp0 & 11 & 6 & 56 \\
\hline 228 & Neu5Aca2-8Neu5Aca2-8Neu5Aca2-3Galb1-4Glcb-Sp0 & 11 & 6 & 53 \\
\hline 590 & Galb1-4GIcNAcb1-3Galb1-4GIcNAcb1-3GalNAca-Sp14 & 11 & 13 & 122 \\
\hline 346 & Neu5Aca2-6Galb1-4GIcNAcb1-2Mana1-6(Mana1-3)Manb1-4GIcNAcb1-4GIcNAc-Sp12 & 11 & 6 & 61 \\
\hline 291 & Galb1-4(Fuca1-3)(6S)GlcNAcb-Sp0 & 10 & 8 & 73 \\
\hline 401 & Fuca1-2Galb1-4GlcNAcb1-3GalNAca-Sp14 & 10 & 8 & 77 \\
\hline 278 & Galb1-3(Fuca1-4)GlcNAcb1-3Galb1-3(Fuca1-4)GlcNAcb-Sp0 & 10 & 4 & 43 \\
\hline 97 & GalNAcb1-3Gala1-4Galb1-4GIcNAcb-Sp0 & 10 & 6 & 57 \\
\hline 359 & KDNa2-3Galb1-4GIc-Sp0 & 10 & 8 & 75 \\
\hline 508 & $\begin{array}{l}\text { GlcNAcb1-6(GlcNAcb1-2)Mana1-6(GlcNAcb1-4)(GlcNAcb1-4(GlcNAcb1-2)Mana1-3)Manb1-4GlcNAcb1-4(Fuca1- } \\
\text { 6)GlcNAc-Sp21 }\end{array}$ & 10 & 8 & 77 \\
\hline 128 & Galb1-3GIcNAcb1-3Galb1-4(Fuca1-3)GIcNAcb-Sp0 & 10 & 2 & 24 \\
\hline 40 & (4S)Galb1-4GIcNAcb-Sp8 & 10 & 10 & 99 \\
\hline 535 & Neu5Aca2-3Galb1-4(Fuca1-3)GlcNAcb1-2Mana-Sp0 & 10 & 8 & 79 \\
\hline 232 & GalNAcb1-4(Neu5Aca2-3)Galb1-4GIcNAcb-Sp0 & 10 & 3 & 28 \\
\hline 381 & Galb1-3GalNAca1-3(Fuca1-2)Galb1-4Glc-Sp0 & 10 & 5 & 54 \\
\hline 200 & Glcb1-6Glcb-Sp8 & 10 & 8 & 84 \\
\hline 212 & Mana1-2Mana1-6(Mana1-3)Mana1-6(Mana1-2Mana1-2Mana1-3)Manb1-4GIcNAcb1-4GIcNAcb-Sp12 & 10 & 6 & 66 \\
\hline 451 & Gala1-3(Fuca1-2)Galb1-4GIcNAcb1-6(Gala1-3(Fuca1-2)Galb1-4GIcNAcb1-3)GalNAc-Sp14 & 10 & 5 & 51 \\
\hline 132 & Galb1-4GIcNAcb1-6GalNAca-Sp8 & 9 & 8 & 81 \\
\hline 514 & (3S)GalNAcb1-4(3S)GlcNAc-Sp8 & 9 & 7 & 78 \\
\hline 85 & (3S)Galb1-4(Fuca1-3)Glcb-Sp0 & 9 & 11 & 118 \\
\hline 268 & Neu5Aca2-6Galb1-4(6S)GlcNAcb-Sp8 & 9 & 7 & 77 \\
\hline 416 & GalNAca1-3GalNAcb1-3Gala1-4Galb1-4Glcb-Sp0 & 9 & 9 & 102 \\
\hline 323 & Neu5Aca2-8Neu5Aca2-8Neu5Acb-Sp8 & 9 & 5 & 60 \\
\hline
\end{tabular}




\begin{tabular}{|c|c|c|c|c|}
\hline 307 & GalNAcb1-3Galb-Sp8 & 9 & 7 & 81 \\
\hline 179 & GlcNAcb1-6(GlcNAcb1-3)Galb1-4GlcNAcb-Sp8 & 9 & 8 & 87 \\
\hline 432 & GlcNAcb1-2Mana1-6(GlcNAcb1-4)(GlcNAcb1-2Mana1-3)Manb1-4GIcNAcb1-4GIcNAc-Sp21 & 9 & 7 & 86 \\
\hline 287 & Neu5Gca2-6Galb1-4GIcNAcb-Sp0 & 9 & 10 & 119 \\
\hline 351 & Galb1-4GIcNAcb1-2Mana1-6Manb1-4GIcNAcb1-4GIcNAc-Sp12 & 9 & 9 & 109 \\
\hline 293 & Galb1-4(Fuca1-3)GlcNAcb1-3Galb1-3(Fuca1-4)GlcNAcb-Sp0 & 8 & 10 & 117 \\
\hline 187 & GlcNAcb1-4-MDPLys & 8 & 10 & 122 \\
\hline 593 & Neu5Aca2-3Galb1-4GIcNAcb1-3Galb1-4GIcNAcb1-3GalNAca-Sp14 & 8 & 13 & 157 \\
\hline 374 & $\begin{array}{l}\text { GalNAca1-3(Fuca1-2)Galb1-3GlcNAcb1-2Mana1-6(GalNAca1-3(Fuca1-2)Galb1-3GlcNAcb1-2Mana1-3)Manb1- } \\
\text { 4GlcNAcb1-4GlcNAcb-Sp20 }\end{array}$ & 8 & 11 & 140 \\
\hline 429 & $\begin{array}{l}\text { Galb1-3GlcNAcb1-6(Galb1-3GlcNAcb1-2)Mana1-6(Galb1-3GlcNAcb1-2Mana1-3)Manb1-4GIcNAcb1-4GlcNAcb- } \\
\text { Sp19 }\end{array}$ & 8 & 6 & 74 \\
\hline 515 & GalNAcb1-4(6S)GIcNAc-Sp8 & 8 & 4 & 54 \\
\hline 216 & Mana1-6(Mana1-3)Mana1-6(Mana1-2Mana1-3)Manb1-4GlcNAcb1-4GIcNAcb-Sp12 & 8 & 4 & 51 \\
\hline 392 & Galb1-3GlcNAcb1-3GalNAca-Sp14 & 8 & 5 & 57 \\
\hline 55 & $\begin{array}{l}\text { Neu5Aca2-6Galb1-4GIcNAcb1-2Mana1-6(Neu5Aca2-6Galb1-4GIcNAcb1-2Mana1-3)Manb1-4GIcNAcb1- } \\
\text { 4GlcNAcb-Sp12 }\end{array}$ & 8 & 4 & 57 \\
\hline 86 & GalNAca1-3(Fuca1-2)Galb1-4GlcNAcb-Sp0 & 8 & 5 & 68 \\
\hline 405 & Gala1-4Galb1-4GIcNAcb1-2Mana1-6(Gala1-4Galb1-4GIcNAcb1-2Mana1-3)Manb1-4GIcNAcb1-4GlcNAcb-Sp24 & 8 & 6 & 83 \\
\hline 5 & GalNAca-Sp15 & 8 & 4 & 50 \\
\hline 130 & Galb1-3(Fuca1-4)GIcNAc-Sp8 & 7 & 5 & 61 \\
\hline 6 & Fuca-Sp8 & 7 & 4 & 54 \\
\hline 24 & (3S)Galb1-4(Fuca1-3)(6S)Glc-Sp0 & 7 & 8 & 113 \\
\hline 342 & GlcNAca1-4Galb1-4GlcNAcb1-3Galb1-4Glcb-Sp0 & 7 & 12 & 155 \\
\hline 125 & Gala1-6Glcb-Sp8 & 7 & 8 & 103 \\
\hline 236 & Neu5Aca2-6(Neu5Aca2-3)GalNAca-Sp8 & 7 & 9 & 124 \\
\hline 414 & Gala1-3(Fuca1-2)Galb1-4GIcNAcb1-3GalNAca-Sp14 & 7 & 5 & 70 \\
\hline 536 & GlcNAcb1-3Galb1-4GIcNAcb1-6(GIcNAcb1-3)Galb1-4GIcNAc-Sp0 & 7 & 6 & 92 \\
\hline 500 & Fuca1-2Galb1-4GIcNAcb1-2Mana-Sp0 & 7 & 4 & 58 \\
\hline 408 & Galb1-3GIcNAca1-6Galb1-4GIcNAcb-Sp0 & 6 & 8 & 133 \\
\hline
\end{tabular}




\begin{tabular}{|c|c|c|c|c|}
\hline 269 & Neu5Aca2-6Galb1-4GIcNAcb-Sp0 & 6 & 6 & 96 \\
\hline 233 & GalNAcb1-4(Neu5Aca2-3)Galb1-4GIcNAcb-Sp8 & 6 & 9 & 144 \\
\hline 464 & $\begin{array}{l}\text { Neu5Aca2-6Galb1-4GIcNAcb1-2Mana1-6(GIcNAcb1-4)(Neu5Aca2-6Galb1-4GIcNAcb1-2Mana1-3)Manb1- } \\
\text { 4GIcNAcb1-4GIcNAcb-Sp21 }\end{array}$ & 6 & 4 & 68 \\
\hline 168 & Galb1-4GIcNAcb1-6(Galb1-3)GalNAc-Sp14 & 6 & 2 & 35 \\
\hline 176 & GIcNAcb1-2Galb1-3GalNAca-Sp8 & 6 & 8 & 140 \\
\hline 485 & Mana1-6(Mana1-3)Manb1-4GlcNAcb1-4(Fuca1-6)GlcNAcb-Sp19 & 6 & 2 & 26 \\
\hline 240 & Neu5Aca2-3Galb1-3(Fuca1-4)GlcNAcb-Sp8 & 5 & 13 & 245 \\
\hline 378 & Neu5Aca2-6Galb1-4GIcNAcb1-3GalNAc-Sp14 & 5 & 5 & 87 \\
\hline 49 & Neu5,9Ac2a2-6Galb1-4GlcNAcb-Sp8 & 5 & 4 & 74 \\
\hline 131 & Fuca1-4(Galb1-3)GlcNAcb-Sp8 & 5 & 7 & 127 \\
\hline 43 & (6S)Galb1-4Glcb-Sp8 & 5 & 7 & 148 \\
\hline 380 & GalNAcb1-4GIcNAcb1-2Mana1-6(GalNAcb1-4GIcNAcb1-2Mana1-3)Manb1-4GIcNAcb1-4GIcNAc-Sp12 & 5 & 4 & 84 \\
\hline 231 & Neu5Aca2-3(6S)Galb1-4(Fuca1-3)GlcNAcb-Sp8 & 5 & 5 & 96 \\
\hline 520 & GalNAca1-3(Fuca1-2)Galb1-4GIcNAcb1-6GalNAc-Sp14 & 5 & 3 & 64 \\
\hline 210 & Mana1-2Mana1-3Mana-Sp9 & 5 & 6 & 120 \\
\hline 2 & Glca-Sp8 & 4 & 5 & 121 \\
\hline 355 & Galb1-3GlcNAcb1-2Mana1-6(Galb1-3GlcNAcb1-2Mana1-3)Manb1-4GIcNAcb1-4(Fuca1-6)GlcNAcb-Sp22 & 4 & 7 & 164 \\
\hline 63 & Fuca1-2Galb1-3GalNAca-Sp14 & 4 & 6 & 129 \\
\hline 517 & (4S)GalNAcb-Sp10 & 4 & 2 & 42 \\
\hline 87 & GalNAca1-3(Fuca1-2)Galb1-4GIcNAcb-Sp8 & 4 & 4 & 102 \\
\hline 150 & Galb1-3GlcNAcb-Sp0 & 4 & 9 & 232 \\
\hline 113 & Gala1-3GalNAcb-Sp8 & 4 & 3 & 85 \\
\hline 136 & Neu5Aca2-6(Galb1-3)GalNAca-Sp8 & 4 & 8 & 213 \\
\hline 407 & Galb1-3GlcNAcb1-6Galb1-4GlcNAcb-Sp0 & 3 & 5 & 154 \\
\hline 94 & GalNAca1-4(Fuca1-2)Galb1-4GIcNAcb-Sp8 & 3 & 6 & 199 \\
\hline 16 & GIcNAcb-Sp0 & 2 & 6 & 299 \\
\hline 4 & GalNAca-Sp8 & 1 & 9 & 779 \\
\hline
\end{tabular}

Supporting data 2. AAL-100ug_14900_V5.0_DATA

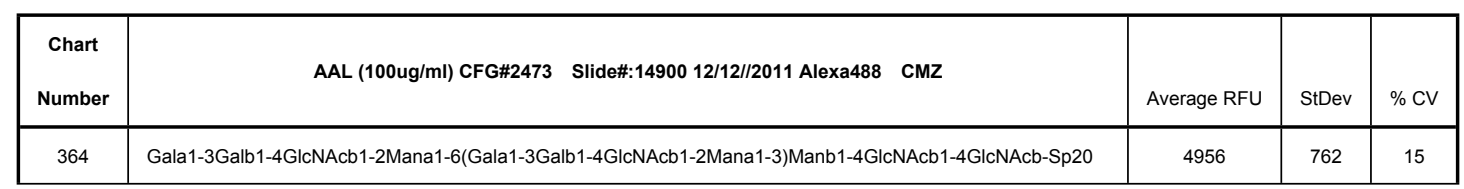




\begin{tabular}{|c|c|c|c|c|}
\hline 561 & Gala1-3Galb1-4GIcNAcb1-2Mana1-6(Gala1-3Galb1-4GIcNAcb1-2Mana1-3)Manb1-4GIcNAcb1-4GIcNAc-Sp24 & 3883 & 316 & 8 \\
\hline 461 & $\begin{array}{l}\text { Neu5Aca2-3Galb1-4GlcNAcb1-4Mana1-6(GlcNAcb1-4)(Neu5Aca2-3Galb1-4GlcNAcb1-4(Neu5Aca2-3Galb1- } \\
\text { 4GlcNAcb1-2)Mana1-3)Manb1-4GlcNAcb1-4GIcNAcb-Sp21 }\end{array}$ & 3763 & 571 & 15 \\
\hline 29 & (3S)Galb1-3GalNAca-Sp8 & 3140 & 198 & 6 \\
\hline 484 & $\begin{array}{l}\text { Neu5Aca2-3Galb1-4GIcNAcb1-2Mana1-6(Neu5Aca2-3Galb1-4GIcNAcb1-2Mana1-3)Manb1-4GlcNAcb1-4(Fuca1- } \\
\text { 6)GIcNAcb-Sp24 }\end{array}$ & 2379 & 96 & 4 \\
\hline 34 & (3S)Galb1-4(6S)GIcNAcb-Sp0 & 1724 & 441 & 26 \\
\hline 463 & $\begin{array}{l}\text { Neu5Aca2-3Galb1-4GlcNAcb1-6(Neu5Aca2-3Galb1-4GlcNAcb1-2)Mana1-6(GlcNAcb1-4)(Neu5Aca2-3Galb1- } \\
\text { 4GlcNAcb1-4(Neu5Aca2-3Galb1-4GlcNAcb1-2)Mana1-3)Manb1-4GlcNAcb1-4GlcNAcb-Sp21 }\end{array}$ & 1637 & 234 & 14 \\
\hline 556 & Neu5Gca2-8Neu5Gca2-3Galb1-4GIcNAcb1-3Galb1-4GIcNAc-Sp0 & 1502 & 302 & 20 \\
\hline 570 & (3S)GlcAb1-3Galb1-4GlcNAcb1-3Galb1-4GIc-Sp0 & 1081 & 127 & 12 \\
\hline 460 & $\begin{array}{l}\text { Neu5Aca2-3Galb1-4GlcNAcb1-2Mana1-6(GlcNAcb1-4)(Neu5Aca2-3Galb1-4GlcNAcb1-2Mana1-3)Manb1- } \\
\text { 4GIcNAcb1-4GIcNAcb-Sp21 }\end{array}$ & 1042 & 182 & 17 \\
\hline 462 & $\begin{array}{l}\text { Neu5Aca2-3Galb1-4GlcNAcb1-6(Neu5Aca2-3Galb1-4GIcNAcb1-2)Mana1-6(GlcNAcb1-4)(Neu5Aca2-3Galb1- } \\
\text { 4GlcNAcb1-2Mana1-3)Manb1-4GlcNAcb1-4GlcNAcb-Sp21 }\end{array}$ & 934 & 98 & 10 \\
\hline 26 & (3S)Galb1-4(6S)Glcb-Sp0 & 824 & 64 & 8 \\
\hline 116 & Gala1-3Galb1-4GIcNAcb-Sp8 & 820 & 64 & 8 \\
\hline 522 & Gala1-3Galb1-4GlcNAcb1-2Mana-Sp0 & 790 & 85 & 11 \\
\hline 27 & (3S)Galb1-4(6S)GIcb-Sp8 & 772 & 115 & 15 \\
\hline 37 & (3S)Galb1-4GlcNAcb-Sp8 & 770 & 62 & 8 \\
\hline 247 & Neu5Aca2-3Galb1-3GalNAcb1-3Gala1-4Galb1-4GIcb-Sp0 & 743 & 66 & 9 \\
\hline 36 & (3S)Galb1-4GlcNAcb-Sp0 & 648 & 54 & 8 \\
\hline 259 & Neu5Aca2-3Galb1-4GIcNAcb1-3Galb1-4GIcNAcb1-3Galb1-4GIcNAcb-Sp0 & 644 & 240 & 37 \\
\hline 224 & Neu5Aca2-3Galb1-3GalNAca-Sp8 & 580 & 65 & 11 \\
\hline 554 & Neu5Aca2-8Neu5Gca2-3Galb1-4GIcNAc-Sp0 & 505 & 128 & 25 \\
\hline 544 & $\begin{array}{l}\text { Neu5Gca2-3Galb1-4GIcNAcb1-3Galb1-4GIcNAcb1-2Mana1-6(Neu5Gca2-3Galb1-4GlcNAcb1-3Galb1- } \\
\text { 4GIcNAcb1-2Mana1-3)Manb1-4GIcNAcb1-4GIcNAcb-Sp24 }\end{array}$ & 473 & 28 & 6 \\
\hline 326 & $\begin{array}{l}\text { Neu5Aca2-3Galb1-4GIcNAcb1-2Mana1-6(Neu5Aca2-3Galb1-4GIcNAcb1-2Mana1-3)Manb1-4GIcNAcb1- } \\
\text { 4GIcNAcb-Sp12 }\end{array}$ & 359 & 12 & 3 \\
\hline 553 & Neu5Gca2-8Neu5Gca2-3Galb1-4GIcNAc-Sp0 & 333 & 94 & 28 \\
\hline 406 & Gala1-3Galb1-4GIcNAcb1-3GalNAca-Sp14 & 288 & 50 & 17 \\
\hline 319 & $\begin{array}{l}\text { Neu5Aca2-6Galb1-4GIcNAcb1-2Mana1-6(Neu5Aca2-3Galb1-4GIcNAcb1-2Mana1-3)Manb1-4GlcNAcb1- } \\
\text { 4GlcNAcb-Sp12 }\end{array}$ & 268 & 75 & 28 \\
\hline 318 & Neu5Aca2-3Galb1-4GIcNAcb1-6(Neu5Aca2-3Galb1-3)GalNAca-Sp14 & 241 & 44 & 18 \\
\hline 296 & Neu5Aca2-3Galb1-4GIcNAcb1-3Galb1-3GIcNAcb-Sp0 & 199 & 32 & 16 \\
\hline 25 & (3S)Galb1-4Glcb-Sp8 & 166 & 45 & 27 \\
\hline 30 & (3S)Galb1-3GlcNAcb-Sp0 & 148 & 57 & 39 \\
\hline 601 & Neu5Aca2-3Galb1-4GlcNAcb1-3Galb1-4GlcNAcb1-6(Galb1-3)GalNAca-Sp14 & 137 & 34 & 25 \\
\hline 235 & Neu5Aca2-3Galb1-3GalNAcb1-4(Neu5Aca2-3)Galb1-4Glcb-Sp0 & 133 & 68 & 51 \\
\hline 243 & Neu5Aca2-3Galb1-3(6S)GalNAca-Sp8 & 127 & 52 & 41 \\
\hline 605 & GlcNAcb1-6(Neu5Aca2-3Galb1-3)GalNAca-Sp14 & 126 & 26 & 21 \\
\hline 35 & (3S)Galb1-4(6S)GlcNAcb-Sp8 & 122 & 39 & 32 \\
\hline 262 & Neu5Aca2-3Galb1-4GlcNAcb1-3Galb1-4GIcNAcb-Sp0 & 114 & 66 & 58 \\
\hline
\end{tabular}




\begin{tabular}{|c|c|c|c|c|}
\hline 558 & Neu5Aca2-8Neu5Aca2-3Galb1-4GlcNAc-Sp0 & 86 & 13 & 15 \\
\hline 118 & Gala1-3Galb1-4GIc-Sp10 & 83 & 57 & 68 \\
\hline 490 & Gala1-3Galb1-3GlcNAcb1-6GalNAca-Sp14 & 83 & 15 & 18 \\
\hline 555 & Neu5Gca2-8Neu5Aca2-3Galb1-4GlcNAc-Sp0 & 66 & 10 & 15 \\
\hline 571 & (3S)GlcAb1-3Galb1-4GIcNAcb1-2Mana-Sp0 & 60 & 14 & 23 \\
\hline 110 & Gala1-4(Gala1-3)Galb1-4GlcNAcb-Sp8 & 54 & 25 & 45 \\
\hline 585 & $\begin{array}{l}\text { Galb1-4GIcNAcb1-3Galb1-4GIcNAcb1-3Galb1-4GlcNAcb1-6(Galb1-4GIcNAcb1-3Galb1-4GIcNAcb1-3Galb1- } \\
\text { 4GlcNAb1-2)Mana1-6(Galb1-4GlcNAcb1-3Galb1-4GIcNAcb1-3Galb1-4GIcNAcb1-2Mana1-3)Manb1-4GIcNAcb1- } \\
\text { 4(Fuca1-6)GlcNAcb-Sp24 }\end{array}$ & 51 & 15 & 30 \\
\hline 133 & Galb1-4GIcNAcb1-6GalNAc-Sp14 & 50 & 50 & 100 \\
\hline 284 & Neu5Gca2-3Galb1-4GIcNAcb-Sp0 & 48 & 10 & 21 \\
\hline 538 & Gala1-3(Fuca1-2)Galb1-3GalNAcb1-3Gala1-4Galb1-4GIc-Sp21 & 44 & 9 & 20 \\
\hline 589 & $\begin{array}{l}\text { Galb1-4GlcNAcb1-3Galb1-4GlcNAcb1-3Galb1-4GlcNAcb1-3Galb1-4GIcNAcb1-3Galb1-4GlcNAcb1-6(Galb1- } \\
\text { 4GlcNAcb1-3Galb1-4GlcNAcb1-3Galb1-4GlcNAcb1-3Galb1-4GlcNAcb1-3Galb1-4GlcNAb1-2)Mana1-6(Galb1- } \\
\text { 4GlcNAcb1-3Galb1-4GlcNAcb1-3Galb1-4GlcNAcb1-3Galb1-4GlcNAcb1-3Galb1-4GlcNAcb1-2Mana1-3)Manb1- } \\
\text { 4GlcNAcb1-4(Fuca1-6)GlcNAcb-Sp24 }\end{array}$ & 43 & 18 & 41 \\
\hline 327 & $\begin{array}{l}\text { Neu5Aca2-3Galb1-4GlcNAcb1-2Mana1-6(Neu5Aca2-6Galb1-4GlcNAcb1-2Mana1-3)Manb1-4GlcNAcb1- } \\
\text { 4GlcNAcb-Sp12 }\end{array}$ & 42 & 15 & 35 \\
\hline 587 & $\begin{array}{l}\text { Galb1-4GIcNAcb1-3Galb1-4GlcNAcb1-3Galb1-4GIcNAcb1-3Galb1-4GlcNAcb1-6(Galb1-4GlcNAcb1-3Galb1- } \\
\text { 4GIcNAcb1-3Galb1-4GlcNAcb1-3Galb1-4GlcNAb1-2)Mana1-6(Galb1-4GIcNAcb1-3Galb1-4GlcNAcb1-3Galb1- } \\
\text { 4GIcNAcb1-3Galb1-4GlcNAcb1-2Mana1-3)Manb1-4GlcNAcb1-4(Fuca1-6)GlcNAcb-Sp24 }\end{array}$ & 41 & 15 & 37 \\
\hline 396 & $\begin{array}{l}\text { Gala1-3Galb1-3(Fuca1-4)GIcNAcb1-2Mana1-6(Gala1-3Galb1-3(Fuca1-4)GIcNAcb1-2Mana1-3)Manb1- } \\
\text { 4GIcNAcb1-4GIcNAc-Sp19 }\end{array}$ & 38 & 19 & 50 \\
\hline 509 & $\begin{array}{l}\text { Galb1-4GIcNAcb1-6(Galb1-4GIcNAcb1-2)Mana1-6(GlcNAcb1-4)Galb1-4GIcNAcb1-4(Gal b1-4GIcNAcb1- } \\
\text { 2)Mana1-3)Manb1-4GlcNAcb1-4(Fuca1-6)GlcNAc-Sp21 }\end{array}$ & 38 & 6 & 15 \\
\hline 31 & (3S)Galb1-3GlcNAcb-Sp8 & 38 & 26 & 68 \\
\hline 566 & $\begin{array}{l}\text { Galb1-4GIcNAcb1-3Galb1-4GlcNAcb1-3Galb1-4GIcNAcb1-3Galb1-4GlcNAcb1-3Galb1-4GIcNAcb1-2Mana1- } \\
\text { 6(Galb1-4GlcNAcb1-3Galb1-4GIcNAcb1-3Galb1-4GIcNAcb1-3Galb1-4GIcNAcb1-3Galb1-4GIcNAcb1-2Mana1- } \\
\text { 3)Manb1-4GIcNAcb1-4GlcNAcb-Sp25 }\end{array}$ & 37 & 9 & 25 \\
\hline 368 & Neu5Aca2-6GlcNAcb1-4GIcNAcb1-4GlcNAc-Sp21 & 36 & 24 & 65 \\
\hline 542 & $\begin{array}{l}\text { Galb1-4GIcNAcb1-3Galb1-4GIcNAcb1-2Mana1-6(Galb1-4GlcNAcb1-3Galb1-4GIcNAcb1-2Mana1-3)Manb1- } \\
\text { 4GlcNAcb1-4GlcNAcb-Sp12 }\end{array}$ & 35 & 9 & 25 \\
\hline 5 & GalNAca-Sp15 & 35 & 20 & 57 \\
\hline 74 & Fuca1-2Galb1-4GlcNAcb1-3Galb1-4GIcNAcb-Sp0 & 34 & 12 & 36 \\
\hline 98 & GalNAcb1-4(Fuca1-3)GlcNAcb-Sp0 & 34 & 14 & 43 \\
\hline 24 & (3S)Galb1-4(Fuca1-3)(6S)Glc-Sp0 & 34 & 14 & 43 \\
\hline 107 & Gala1-3(Fuca1-2)Galb1-4GIcb-Sp0 & 33 & 12 & 36 \\
\hline 196 & Glca1-4Glcb-Sp8 & 32 & 15 & 47 \\
\hline 404 & Gala1-4Galb1-3GIcNAcb1-2Mana1-6(Gala1-4Galb1-3GlcNAcb1-2Mana1-3)Manb1-4GIcNAcb1-4GIcNAcb-Sp19 & 32 & 4 & 13 \\
\hline 261 & Neu5Aca2-3Galb1-4GlcNAcb-Sp8 & 32 & 16 & 51 \\
\hline 207 & KDNa2-3Galb1-4GIcNAcb-Sp0 & 31 & 27 & 88 \\
\hline 167 & Galb1-4GlcNAcb1-6(Galb1-3)GalNAca-Sp8 & 31 & 18 & 60 \\
\hline
\end{tabular}




\begin{tabular}{|c|c|c|c|c|}
\hline 580 & $\begin{array}{l}\text { Galb1-4GlcNAcb1-3Galb1-4GlcNAcb1-3Galb1-4GIcNAcb1-3Galb1-4GIcNAcb1-2Mana1-6(Galb1-4GlcNAcb1- } \\
\text { 3Galb1-4GlcNAcb1-3Galb1-4GIcNAcb1-3Galb1-4GIcNAcb1-2Mana1-3)Manb1-4GlcNAcb1-4(Fuca1-6)GlcNAcb- } \\
\text { Sp24 }\end{array}$ & 31 & 12 & 40 \\
\hline 395 & Gala1-3Galb1-3GIcNAcb1-2Mana1-6(Gala1-3Galb1-3GIcNAcb1-2Mana1-3)Manb1-4GIcNAcb1-4GlcNAc-Sp19 & 31 & 19 & 63 \\
\hline 486 & $\begin{array}{l}\text { Galb1-4GIcNAcb1-6(Galb1-4GIcNAcb1-2)Mana1-6(Galb1-4GlcNAcb1-2Mana1-3)Manb1-4GIcNAcb1-4(Fuca1- } \\
\text { 6)GIcNAcb-Sp24 }\end{array}$ & 30 & 15 & 49 \\
\hline 574 & Neu5Aca2-8Neu5Aca2-3Galb1-3GalNAcb1-4(Neu5Aca2-3)Galb1-4Glc-Sp21 & 30 & 9 & 31 \\
\hline 593 & Neu5Aca2-3Galb1-4GIcNAcb1-3Galb1-4GIcNAcb1-3GalNAca-Sp14 & 30 & 10 & 33 \\
\hline 604 & $\begin{array}{l}\text { Neu5Aca2-3Galb1-4GlcNAcb1-3Galb1-4GIcNAcb1-2Mana1-6(Neu5Aca2-3Galb1-4GlcNAcb1-3Galb1- } \\
\text { 4GIcNAcb1-2Mana1-3)Manb1-4GlcNAcb1-4GlcNAcb-Sp12 }\end{array}$ & 30 & 5 & 15 \\
\hline 445 & (6S)Galb1-3(6S)GIcNAc-Sp0 & 29 & 15 & 50 \\
\hline 374 & $\begin{array}{l}\text { GalNAca1-3(Fuca1-2)Galb1-3GIcNAcb1-2Mana1-6(GalNAca1-3(Fuca1-2)Galb1-3GlcNAcb1-2Mana1-3)Manb1- } \\
\text { 4GlcNAcb1-4GlcNAcb-Sp20 }\end{array}$ & 29 & 19 & 66 \\
\hline 117 & Gala1-3Galb1-4GIcb-Sp0 & 29 & 9 & 30 \\
\hline 82 & Fucb1-3GlcNAcb-Sp8 & 29 & 21 & 73 \\
\hline 502 & Fuca1-2(6S)Galb1-3(6S)GlcNAcb-Sp0 & 29 & 8 & 29 \\
\hline 338 & Neu5Aca2-3Galb1-4(Fuca1-3)GIcNAcb1-6(Neu5Aca2-3Galb1-3)GalNAc-Sp14 & 29 & 19 & 66 \\
\hline 595 & GlcNAcb1-3Galb1-4GlcNAcb1-6(Galb1-3)GalNAca-Sp14 & 29 & 11 & 37 \\
\hline 477 & Galb1-3GIcNAcb1-2Mana1-6(GIcNAcb1-4)(Galb1-3GIcNAcb1-2Mana1-3)Manb1-4GIcNAcb1-4GIcNAcb-Sp21 & 29 & 15 & 54 \\
\hline 11 & Neu5Acb-Sp8 & 28 & 17 & 62 \\
\hline 469 & Gala1-3(Fuca1-2)Galb1-3GalNAcb-Sp8 & 28 & 13 & 45 \\
\hline 583 & $\begin{array}{l}\text { Galb1-4GIcNAcb1-3Galb1-4GlcNAcb1-6(Galb1-4GlcNAcb1-3Galb1-4GlcNAb1-2)Mana1-6(Galb1-4GlcNAcb1- } \\
\text { 3Galb1-4GlcNAcb1-2Mana1-3)Manb1-4GlcNAcb1-4(Fuca1-6)GlcNAcb-Sp24 }\end{array}$ & 28 & 13 & 47 \\
\hline 10 & Neu5Aca-Sp11 & 28 & 10 & 35 \\
\hline 454 & GalNAcb1-4Galb1-4GIcb-Sp0 & 28 & 16 & 58 \\
\hline 565 & $\begin{array}{l}\text { GlcNAcb1-3Galb1-4GlcNAcb1-3Galb1-4GlcNAcb1-3Galb1-4GlcNAcb1-3Galb1-4GIcNAcb1-2Mana1-6(GlcNAcb1- } \\
\text { 3Galb1-4GlcNAcb1-3Galb1-4GIcNAcb1-3Galb1-4GlcNAcb1-3Galb1-4GlcNAcb1-2Mana1-3)Manb1-4GIcNAcb1- } \\
\text { 4GlcNAcb-Sp25 }\end{array}$ & 27 & 13 & 47 \\
\hline 491 & Galb1-3(Fuca1-4)GIcNAcb1-6GalNAca-Sp14 & 27 & 12 & 43 \\
\hline 533 & $\begin{array}{l}\text { Galb1-4GIcNAcb1-2 Mana1-6(Galb1-4GlcNAcb1-4)(Galb1-4GIcNAcb1-2Mana1-3)Manb1-4GlcNAcb1-4(Fuca1- } \\
\text { 6)GIcNAc-Sp21 }\end{array}$ & 27 & 17 & 63 \\
\hline 598 & Neu5Aca2-6Galb1-4GIcNAcb1-3Galb1-4GIcNAcb1-3GalNAca-Sp14 & 27 & 16 & 58 \\
\hline 315 & Mana1-6(Mana1-3)Mana1-6(Mana1-3)Manb-Sp10 & 27 & 6 & 23 \\
\hline 503 & Neu5Aca2-6GalNAcb1-4(6S)GlcNAcb-Sp8 & 26 & 16 & 62 \\
\hline 532 & $\begin{array}{l}\text { Galb1-4GIcNAcb1-2 Mana1-6(GIcNAcb1-4)(Galb1-4GIcNAcb1-2Mana1-3)Manb1-4GIcNAcb1-4(Fuca1-6)GIcNAc- } \\
\text { Sp21 }\end{array}$ & 26 & 15 & 56 \\
\hline 8 & Rhaa-Sp8 & 26 & 27 & 103 \\
\hline 577 & $\begin{array}{l}\text { GlcNAcb1-3Galb1-4GlcNAcb1-3Galb1-4GlcNAcb1-2Mana1-6(GlcNAcb1-3Galb1-4GlcNAcb1-3Galb1-4GlcNAcb1- } \\
\text { 2Mana1-3)Manb1-4GIcNAcb1-4(Fuca1-6)GlcNAcb-Sp24 }\end{array}$ & 26 & 4 & 17 \\
\hline 148 & Galb1-3GIcNAcb1-3Galb1-4GIcNAcb-Sp0 & 26 & 13 & 52 \\
\hline 581 & $\begin{array}{l}\text { GlcNAcb1-3Galb1-4GIcNAcb1-3Galb1-4GlcNAcb1-3Galb1-4GIcNAcb1-3Galb1-4GlcNAcb1-2Mana1-6(GlcNAcb1- } \\
\text { 3Galb1-4GlcNAcb1-3Galb1-4GlcNAcb1-3Galb1-4GlcNAcb1-3Galb1-4GlcNAcb1-2Mana1-3)Manb1-4GIcNAcb1- } \\
\text { 4(Fuca1-6)GlcNAcb-Sp19 }\end{array}$ & 26 & 13 & 50 \\
\hline
\end{tabular}




\begin{tabular}{|c|c|c|c|c|}
\hline 126 & Galb1-2Galb-Sp8 & 25 & 13 & 53 \\
\hline 405 & Gala1-4Galb1-4GIcNAcb1-2Mana1-6(Gala1-4Galb1-4GIcNAcb1-2Mana1-3)Manb1-4GIcNAcb1-4GIcNAcb-Sp24 & 25 & 10 & 41 \\
\hline 236 & Neu5Aca2-6(Neu5Aca2-3)GalNAca-Sp8 & 25 & 20 & 79 \\
\hline 421 & $\begin{array}{l}\text { Fuca1-2Galb1-4GIcNAcb1-2Mana1-6(Fuca1-2Galb1-4GIcNAcb1-2Mana1-3)Manb1-4GlcNAcb1-4(Fuca1- } \\
\text { 6)GIcNAcb-Sp22 }\end{array}$ & 25 & 13 & 50 \\
\hline 564 & GalNAcb1-4GIcNAcb1-3GalNAcb1-4GIcNAcb-Sp0 & 25 & 14 & 57 \\
\hline 271 & Neu5Aca2-6Galb1-4GIcNAcb1-3Galb1-4(Fuca1-3)GIcNAcb1-3Galb1-4(Fuca1-3)GlcNAcb-Sp0 & 25 & 15 & 60 \\
\hline 343 & GlcNAca1-4Galb1-4GIcNAcb1-3Galb1-4(Fuca1-3)GIcNAcb1-3Galb1-4(Fuca1-3)GIcNAcb-Sp0 & 24 & 11 & 43 \\
\hline 58 & $\begin{array}{l}\text { Neu5Aca2-6Galb1-4GlcNAcb1-2Mana1-6(Neu5Aca2-6Galb1-4GlcNAcb1-2Mana1-3)Manb1-4GlcNAcb1- } \\
\text { 4GlcNAcb-Sp24 }\end{array}$ & 24 & 14 & 57 \\
\hline 608 & $\begin{array}{l}\text { Neu5Aca2-3Galb1-4GIcNAcb1-3Galb1-4GIcNAcb1-3Galb1-4GlcNAcb1-2Mana1-6(Neu5Aca2-3Galb1- } \\
\text { 4GlcNAcb1-3Galb1-4GIcNAcb1-3Galb1-4GIcNAcb1-2Mana1-3)Manb1-4GIcNAcb1-4GlcNAcb-Sp12 }\end{array}$ & 24 & 13 & 55 \\
\hline 291 & Galb1-4(Fuca1-3)(6S)GIcNAcb-Sp0 & 24 & 8 & 35 \\
\hline 47 & (6S)GlcNAcb-Sp8 & 24 & 20 & 84 \\
\hline 285 & Neu5Gca2-3Galb1-4GIcb-Sp0 & 24 & 10 & 42 \\
\hline 546 & $\begin{array}{l}\text { GlcNAcb1-3Galb1-4GIcNAcb1-3Galb1-4GlcNAcb1-2Mana1-6(GlcNAcb1-3Galb1-4GlcNAcb1-3Galb1-4GlcNAcb1- } \\
\text { 2Mana1-3)Manb1-4GlcNAcb1-4GIcNAcb-Sp12 }\end{array}$ & 24 & 13 & 55 \\
\hline 104 & Gala1-3(Fuca1-2)Galb1-4(Fuca1-3)GlcNAcb-Sp0 & 23 & 14 & 59 \\
\hline 407 & Galb1-3GIcNAcb1-6Galb1-4GIcNAcb-Sp0 & 23 & 12 & 53 \\
\hline 226 & GalNAcb1-4(Neu5Aca2-8Neu5Aca2-8Neu5Aca2-8Neu5Aca2-3)Galb1-4Glcb-Sp0 & 23 & 2 & 7 \\
\hline 573 & $\begin{array}{l}\text { Galb1-3GlcNAcb1-3Galb1-4GlcNAcb1-6(Galb1-3GlcNAcb1-3Galb1-4GlcNAb1-2)Mana1-6(Galb1-3GlcNAcb1- } \\
\text { 3Galb1-4GlcNAcb1-2Mana1-3)Manb1-4GlcNAcb1-4(Fuca1-6)GlcNAcb-Sp24 }\end{array}$ & 23 & 8 & 33 \\
\hline 93 & GalNAca1-3Galb-Sp8 & 23 & 11 & 46 \\
\hline 14 & Manb-Sp8 & 23 & 12 & 52 \\
\hline 378 & Neu5Aca2-6Galb1-4GlcNAcb1-3GalNAc-Sp14 & 23 & 12 & 54 \\
\hline 576 & $\begin{array}{l}\text { Galb1-4GlcNAcb1-3Galb1-4GlcNAcb1-2Mana1-6(Galb1-4GlcNAcb1-3Galb1-4GlcNAcb1-2Mana1-3)Manb1- } \\
\text { 4GlcNAcb1-4(Fuca1-6)GlcNAcb-Sp24 }\end{array}$ & 23 & 12 & 50 \\
\hline 78 & Fuca1-2Galb1-4Glcb-Sp0 & 23 & 15 & 66 \\
\hline 114 & Gala1-3Galb1-4(Fuca1-3)GlcNAcb-Sp8 & 23 & 17 & 75 \\
\hline 450 & Fuca1-2Galb1-4GIcNAcb1-6(Fuca1-2Galb1-4GIcNAcb1-3)GalNAc-Sp14 & 23 & 5 & 22 \\
\hline 121 & Gala1-4Galb1-4GlcNAcb-Sp0 & 23 & 15 & 65 \\
\hline 442 & Neu5Aca2-3Galb1-4GIcNAcb1-3Galb-Sp8 & 23 & 15 & 68 \\
\hline 563 & GalNAcb1-3GlcNAcb-Sp0 & 22 & 10 & 47 \\
\hline 143 & Galb1-3GalNAcb-Sp8 & 22 & 17 & 76 \\
\hline 92 & GalNAca1-3GalNAcb-Sp8 & 22 & 9 & 39 \\
\hline 515 & GalNAcb1-4(6S)GIcNAc-Sp8 & 22 & 15 & 70 \\
\hline 597 & $\begin{array}{l}\text { Neu5Aca2-3Galb1-4GIcNAcb1-3Galb1-4GIcNAcb1-6(Neu5Aca2-3Galb1-4GIcNAcb1-3Galb1-4GIcNAcb1- } \\
\text { 3)GalNAca-Sp14 }\end{array}$ & 22 & 2 & 8 \\
\hline 592 & Galb1-4GIcNAcb1-3Galb1-4GIcNAcb1-6(Galb1-4GIcNAcb1-3Galb1-4GIcNAcb1-3)GalNAca-Sp14 & 22 & 6 & 25 \\
\hline 366 & $\begin{array}{l}\text { Fuca1-4(Galb1-3)GlcNAcb1-2Mana1-6(Fuca1-4(Galb1-3)GlcNAcb1-2Mana1-3)Manb1-4GlcNAcb1-4(Fuca1- } \\
\text { 6)GlcNAcb-Sp22 }\end{array}$ & 22 & 14 & 66 \\
\hline 415 & GalNAca1-3(Fuca1-2)Galb1-4GIcNAcb1-3GalNAca-Sp14 & 22 & 9 & 40 \\
\hline 286 & Neu5Gca2-6GalNAca-Sp0 & 22 & 4 & 18 \\
\hline
\end{tabular}




\begin{tabular}{|c|c|c|c|c|}
\hline 329 & Neu5,9Ac2a2-3Galb1-4GlcNAcb-Sp0 & 21 & 12 & 57 \\
\hline 426 & Gala1-3Galb1-3GlcNAcb1-3GalNAc-Sp14 & 21 & 7 & 34 \\
\hline 579 & $\begin{array}{l}\text { GlcNAcb1-3Galb1-4GIcNAcb1-3Galb1-4GIcNAcb1-3Galb1-4GlcNAcb1-2Mana1-6(GlcNAcb1-3Galb1-4GIcNAcb1- } \\
\text { 3Galb1-4GIcNAcb1-3Galb1-4GIcNAcb1-2Mana1-3)Manb1-4GIcNAcb1-4(Fuca1-6)GlcNAcb-Sp24 }\end{array}$ & 21 & 9 & 42 \\
\hline 420 & $\begin{array}{l}\text { Galb1-4(Fuca1-3)GlcNAcb1-2Mana1-6(Galb1-4(Fuca1-3)GlcNAcb1-2Mana1-3)Manb1-4GlcNAcb1-4(Fuca1- } \\
\text { 6)GIcNAcb-Sp22 }\end{array}$ & 21 & 14 & 67 \\
\hline 427 & $\begin{array}{l}\text { Fuca1-2Galb1-3GIcNAcb1-2Mana1-6(Fuca1-2Galb1-3GIcNAcb1-2Mana1-3)Manb1-4GlcNAcb1-4(Fuca1- } \\
\text { 6)GlcNAcb-Sp22 }\end{array}$ & 21 & 18 & 84 \\
\hline 102 & Gala1-3(Fuca1-2)Galb1-3GlcNAcb-Sp0 & 21 & 7 & 31 \\
\hline 111 & Gala1-3GalNAca-Sp8 & 21 & 7 & 33 \\
\hline 559 & $\begin{array}{l}\text { GlcNAcb1-3Galb1-4GlcNAcb1-6(GlcNAcb1-3Galb1-4GlcNAcb1-2)Mana1-6(GlcNAcb1-3Galb1-4GlcNAcb1- } \\
\text { 2Man a1-3)Manb1-4GIcNAcb1-4GlcNAc-Sp24 }\end{array}$ & 21 & 13 & 60 \\
\hline 516 & (3S)GalNAcb1-4GIcNAc-Sp8 & 21 & 12 & 60 \\
\hline 568 & Galb1-3GIcNAcb1-6(Galb1-3)GalNAc-Sp14 & 21 & 11 & 53 \\
\hline 249 & Fuca1-2(6S)Galb1-4GIcb-Sp0 & 21 & 13 & 63 \\
\hline 560 & $\begin{array}{l}\text { Galb1-4GlcNAcb1-3Galb1-4GlcNAcb1-6(Galb1-4GlcNAcb1-3Galb1-4GIcNAcb1-2)Mana1-6(Galb1-4GlcNAcb1- } \\
\text { 3Galb1-4GlcNAcb1-2Mana1-3)Mana1-4GlcNAcb1-4GlcNAc-Sp24 }\end{array}$ & 21 & 13 & 64 \\
\hline 292 & Galb1-4(Fuca1-3)(6S)Glcb-Sp0 & 21 & 15 & 71 \\
\hline 362 & Fuca1-2Galb1-4GIcNAcb1-2Mana1-6(Fuca1-2Galb1-4GIcNAcb1-2Mana1-3)Manb1-4GIcNAcb1-4GIcNAcb-Sp20 & 21 & 8 & 39 \\
\hline 164 & Galb1-4GIcNAcb1-3Galb1-4GIcNAcb-Sp0 & 21 & 13 & 64 \\
\hline 314 & Mana1-6Manb-Sp10 & 21 & 9 & 44 \\
\hline 156 & Galb1-4(6S)Glcb-Sp0 & 21 & 9 & 42 \\
\hline 610 & GIcNAcb1-3Fuca-Sp21 & 20 & 14 & 71 \\
\hline 582 & $\begin{array}{l}\text { Galb1-4GIcNAcb1-3Galb1-4GIcNAcb1-3Galb1-4GlcNAcb1-3Galb1-4GlcNAcb1-3Galb1-4GIcNAcb1-2Mana1- } \\
\text { 6(Galb1-4GlcNAcb1-3Galb1-4GIcNAcb1-3Galb1-4GIcNAcb1-3Galb1-4GIcNAcb1-3Galb1-4GIcNAcb1-2Mana1- } \\
\text { 3)Manb1-4GIcNAcb1-4(Fuca1-6)GIcNAcb-Sp19 }\end{array}$ & 20 & 10 & 52 \\
\hline 90 & GalNAca1-3(Fuca1-2)Galb-Sp8 & 20 & 14 & 70 \\
\hline 245 & Neu5Aca2-6(Neu5Aca2-3Galb1-3)GalNAca-Sp14 & 20 & 10 & 48 \\
\hline 119 & Gala1-3Galb-Sp8 & 20 & 11 & 58 \\
\hline 572 & $\begin{array}{l}\text { Galb1-3GlcNAcb1-3Galb1-4GlcNAcb1-3Galb1-4GlcNAcb1-6(Galb1-3GlcNAcb1-3Galb1-4GlcNAcb1-3Galb1- } \\
\text { 4GlcNAb1-2)Mana1-6(Galb1-3GlcNAcb1-3Galb1-4GlcNAcb1-3Galb1-4GIcNAcb1-2Mana1-3)Manb1-4GlcNAcb1- } \\
\text { 4(Fuca1-6)GlcNAcb-Sp24 }\end{array}$ & 20 & 1 & 5 \\
\hline 190 & GIcNAcb1-4GIcNAcb1-4GIcNAcb1-4GIcNAcb1-4GlcNAcb1-4GIcNAcb1-Sp8 & 20 & 16 & 83 \\
\hline 108 & Gala1-3(Fuca1-2)Galb-Sp8 & 20 & 13 & 69 \\
\hline 337 & GalNAca1-3(Fuca1-2)Galb1-4GIcNAcb1-3Galb1-4GIcNAcb1-3Galb1-4GIcNAcb-Sp0 & 20 & 9 & 45 \\
\hline 376 & $\begin{array}{l}\text { Fuca1-4(Fuca1-2Galb1-3)GlcNAcb1-2Mana1-3(Fuca1-4(Fuca1-2Galb1-3)GlcNAcb1-2Mana1-3)Manb1- } \\
\text { 4GlcNAcb1-4GlcNAcb-Sp19 }\end{array}$ & 20 & 12 & 60 \\
\hline 429 & $\begin{array}{l}\text { Galb1-3GIcNAcb1-6(Galb1-3GIcNAcb1-2)Mana1-6(Galb1-3GIcNAcb1-2Mana1-3)Manb1-4GIcNAcb1-4GIcNAcb- } \\
\text { Sp19 }\end{array}$ & 20 & 10 & 52 \\
\hline 340 & GIcNAca1-4Galb1-4GIcNAcb-Sp0 & 20 & 11 & 57 \\
\hline 562 & GIcNAcb1-3Galb1-4GlcNAcb1-6(GIcNAcb1-3Galb1-3)GalNAca-Sp14 & 19 & 21 & 109 \\
\hline 363 & $\begin{array}{l}\text { Fuca1-2Galb1-4(Fuca1-3)GIcNAcb1-2Mana1-6(Fuca1-2Galb1-4(Fuca1-3)GIcNAcb1-2Mana1-3)Manb1- } \\
\text { 4GIcNAcb1-4GIcNAb-Sp20 }\end{array}$ & 19 & 6 & 32 \\
\hline
\end{tabular}




\begin{tabular}{|c|c|c|c|c|}
\hline 449 & Galb1-4GIcNAcb1-2Mana-Sp0 & 19 & 6 & 32 \\
\hline 607 & $\begin{array}{l}\text { Neu5Aca2-6Galb1-4GIcNAcb1-3Galb1-4GIcNAcb1-3Galb1-4GIcNAcb1-2Mana1-6(Neu5Aca2-6Galb1- } \\
\text { 4GIcNAcb1-3Galb1-4GIcNAcb1-3Galb1-4GIcNAcb1-2Mana1-3)Manb1-4GIcNAcb1-4GlcNAcb-Sp12 }\end{array}$ & 19 & 6 & 29 \\
\hline 431 & Fuca1-3GIcNAcb1-6(Galb1-4GIcNAcb1-3)Galb1-4GIc-Sp21 & 19 & 8 & 41 \\
\hline 297 & 4S(3S)Galb1-4GIcNAcb-Sp0 & 19 & 12 & 63 \\
\hline 541 & $\begin{array}{l}\text { GlcNAcb1-3Galb1-4GIcNAcb1-2Mana1-6(GlcNAcb1-3Galb1-4GIcNAcb1-2Mana1-3)Manb1-4GIcNAcb1- } \\
\text { 4GIcNAcb-Sp25 }\end{array}$ & 19 & 10 & 52 \\
\hline 457 & Neu5Aca2-6Galb1-4GIcNAcb1-6(Fuca1-2Galb1-3GIcNAcb1-3)Galb1-4Glc-Sp21 & 19 & 5 & 25 \\
\hline 99 & GalNAcb1-4GlcNAcb-Sp0 & 19 & 15 & 81 \\
\hline 56 & $\begin{array}{l}\text { Neu5Aca2-6Galb1-4GIcNAcb1-2Mana1-6(Neu5Aca2-6Galb1-4GlcNAcb1-2Mana1-3)Manb1-4GIcNAcb1- } \\
\text { 4GIcNAcb-Sp13 }\end{array}$ & 19 & 5 & 25 \\
\hline 347 & Mana1-6(Neu5Aca2-6Galb1-4GIcNAcb1-2Mana1-3)Manb1-4GIcNAcb1-4GIcNAc-Sp12 & 19 & 11 & 60 \\
\hline 428 & $\begin{array}{l}\text { Gala1-3(Fuca1-2)Galb1-4GlcNAcb1-2Mana1-6(Gala1-3(Fuca1-2)Galb1-4GlcNAcb1-2Mana1-3)Manb1- } \\
\text { 4GIcNAcb1-4(Fuca1-6)GlcNAcb-Sp22 }\end{array}$ & 19 & 12 & 64 \\
\hline 578 & $\begin{array}{l}\text { Galb1-4GIcNAcb1-3Galb1-4GIcNAcb1-3Galb1-4GlcNAcb1-2Mana1-6(Galb1-4GlcNAcb1-3Galb1-4GlcNAcb1- } \\
\text { 3Galb1-4GlcNAcb1-2Mana1-3)Manb1-4GlcNAcb1-4(Fuca1-6)GIcNAcb-Sp24 }\end{array}$ & 19 & 6 & 34 \\
\hline 606 & $\begin{array}{l}\text { Neu5Aca2-6Galb1-4GIcNAcb1-3Galb1-4GIcNAcb1-6(Neu5Aca2-6Galb1-4GIcNAcb1-3Galb1-4GlcNAcb1- } \\
\text { 3)GalNAca-Sp14 }\end{array}$ & 19 & 12 & 64 \\
\hline 75 & Fuca1-2Galb1-4GlcNAcb1-3Galb1-4GlcNAcb1-3Galb1-4GIcNAcb-Sp0 & 19 & 11 & 57 \\
\hline 12 & Galb-Sp8 & 19 & 8 & 44 \\
\hline 500 & Fuca1-2Galb1-4GIcNAcb1-2Mana-Sp0 & 19 & 17 & 94 \\
\hline 475 & $\begin{array}{l}\text { Neu5Aca2-3Galb1-3GIcNAcb1-6(Neu5Aca2-3Galb1-4GIcNAcb1-2)Mana1-6(Neu5Aca2-3Galb1-3GIcNAcb1- } \\
\text { 2Mana1-3)Manb1-4GIcNAcb1-4GIcNAcb-Sp19 }\end{array}$ & 18 & 10 & 55 \\
\hline 440 & Galb1-4Galb-Sp10 & 18 & 11 & 62 \\
\hline 103 & Gala1-3(Fuca1-2)Galb1-3GlcNAcb-Sp8 & 18 & 9 & 51 \\
\hline 127 & Galb1-3(Fuca1-4)GIcNAcb1-3Galb1-4(Fuca1-3)GlcNAcb-Sp0 & 18 & 4 & 22 \\
\hline 548 & $\begin{array}{l}\text { Galb1-4GIcNAcb1-3Galb1-4GlcNAcb1-3Galb1-4GIcNAcb1-2Mana1-6(Galb1-4GlcNAcb1-3Galb1-4GIcNAcb1- } \\
\text { 3Galb1-4GIcNAcb1-2Mana1-3)Manb1-4GlcNAcb1-4GIcNAcb-Sp12 }\end{array}$ & 18 & 17 & 96 \\
\hline 168 & Galb1-4GIcNAcb1-6(Galb1-3)GalNAc-Sp14 & 18 & 11 & 63 \\
\hline 328 & $\begin{array}{l}\text { Galb1-4(Fuca1-3)GIcNAcb1-2Mana1-6(Galb1-4(Fuca1-3)GIcNAcb1-2Mana1-3)Manb1-4GIcNAcb1-4GIcNAcb- } \\
\text { Sp20 }\end{array}$ & 18 & 16 & 88 \\
\hline 419 & GalNAca1-3(Fuca1-2)Galb1-4(Fuca1-3)GIcNAcb1-3GalNAc-Sp14 & 18 & 17 & 93 \\
\hline 42 & (6S)Galb1-4GIcb-Sp0 & 18 & 6 & 31 \\
\hline 278 & Galb1-3(Fuca1-4)GlcNAcb1-3Galb1-3(Fuca1-4)GlcNAcb-Sp0 & 18 & 9 & 52 \\
\hline 229 & GalNAcb1-4(Neu5Aca2-8Neu5Aca2-3)Galb1-4Glcb-Sp0 & 18 & 15 & 82 \\
\hline 437 & $\begin{array}{l}\text { Galb1-4GIcNAcb1-2Mana1-6(GlcNAcb1-4)(Galb1-4GIcNAcb1-4(Galb1-4GIcNAcb1-2)Mana1-3)Manb1- } \\
\text { 4GIcNAcb1-4GIcNAc-Sp21 }\end{array}$ & 18 & 15 & 86 \\
\hline 584 & $\begin{array}{l}\text { GlcNAcb1-3Galb1-4GlcNAcb1-3Galb1-4GIcNAcb1-6(GlcNAcb1-3Galb1-4GlcNAcb1-3Galb1-4GlcNAb1-2)Mana1- } \\
\text { 6(GlcNAcb1-3Galb1-4GlcNAcb1-3Galb1-4GIcNAcb1-2Mana1-3)Manb1-4GIcNAcb1-4(Fuca1-6)GIcNAcb-Sp24 }\end{array}$ & 18 & 6 & 34 \\
\hline 265 & Neu5Aca2-3Galb1-4GIcb-Sp8 & 18 & 10 & 54 \\
\hline 17 & GlcNAcb-Sp8 & 18 & 3 & 16 \\
\hline 534 & Fuca1-4(Galb1-3)GIcNAcb1-2 Mana-Sp0 & 18 & 9 & 52 \\
\hline
\end{tabular}




\begin{tabular}{|c|c|c|c|c|}
\hline 375 & $\begin{array}{l}\text { Gala1-3(Fuca1-2)Galb1-3GlcNAcb1-2Mana1-6(Gala1-3(Fuca1-2)Galb1-3GlcNAcb1-2Mana1-3)Manb1- } \\
\text { 4GlcNAcb1-4GlcNAcb-Sp20 }\end{array}$ & 18 & 9 & 52 \\
\hline 122 & Gala1-4Galb1-4GlcNAcb-Sp8 & 17 & 6 & 34 \\
\hline 283 & Neu5Gca2-3Galb1-4(Fuca1-3)GlcNAcb-Sp0 & 17 & 8 & 46 \\
\hline 277 & Neu5Aca2-8Neu5Aca2-3Galb1-4Glcb-Sp0 & 17 & 8 & 47 \\
\hline 496 & Gala1-3Galb1-4GlcNAcb1-6GalNAca-Sp14 & 17 & 17 & 97 \\
\hline 272 & Neu5Aca2-6Galb1-4GIcNAcb1-3Galb1-4GIcNAcb-Sp0 & 17 & 9 & 50 \\
\hline 180 & GlcNAcb1-3GalNAca-Sp8 & 17 & 10 & 57 \\
\hline 372 & $\begin{array}{l}\text { Gala1-3(Fuca1-2)Galb1-4GIcNAcb1-2Mana1-6(Gala1-3(Fuca1-2)Galb1-4GIcNAcb1-2Mana1-3)Manb1- } \\
\text { 4GlcNAcb1-4GIcNAcb-Sp20 }\end{array}$ & 17 & 12 & 70 \\
\hline 244 & Neu5Aca2-6(Neu5Aca2-3Galb1-3)GalNAca-Sp8 & 17 & 8 & 49 \\
\hline 575 & $\begin{array}{l}\text { GlcNAcb1-3Galb1-4GIcNAcb1-2Mana1-6(GlcNAcb1-3Galb1-4GlcNAcb1-2Mana1-3)Manb1-4GIcNAcb1-4(Fuca1- } \\
\text { 6)GlcNAcb-Sp24 }\end{array}$ & 17 & 7 & 38 \\
\hline 172 & Galb1-4GIcb-Sp0 & 17 & 12 & 71 \\
\hline 447 & $\begin{array}{l}\text { Fuca1-2Galb1-4(Fuca1-3)GIcNAcb1-2Mana1-6(Fuca1-2Galb1-4(Fuca1-3)GlcNAcb1-4(Fuca1-2Galb1-4(Fuca1- } \\
\text { 3)GIcNAcb1-2)Mana1-3)Manb1-4GIcNAcb1-4GlcNAcb-Sp12 }\end{array}$ & 17 & 5 & 28 \\
\hline 89 & GIcNAcb1-3Galb1-3GalNAca-Sp8 & 17 & 11 & 66 \\
\hline 603 & Neu5Aca2-6Galb1-4GIcNAcb1-6(Galb1-3)GalNAca-Sp14 & 17 & 8 & 44 \\
\hline 33 & (3S)Galb1-4(Fuca1-3)GlcNAc-Sp8 & 17 & 9 & 55 \\
\hline 169 & Galb1-4GIcNAcb-Sp0 & 17 & 5 & 32 \\
\hline 569 & $\begin{array}{l}\text { Galb1-4GlcNAcb1-3Galb1-4GlcNAcb1-3Galb1-4GlcNAcb1-3Galb1-4GlcNAcb1-3Galb1-4GlcNAcb1-3Galb1- } \\
\text { 4GlcNAcb1-2Mana1-6(Galb1-4GlcNAcb1-3Galb1-4GlcNAcb1-3Galb1-4GlcNAcb1-3Galb1-4GlcNAcb1-3Galb1- } \\
\text { 4GlcNAcb1-3Galb1-4GlcNAcb1-2Mana1-3)Manb1-4GlcNAcb1-4GlcNAcb-Sp25 }\end{array}$ & 17 & 6 & 34 \\
\hline 529 & GalNAcb1-4GIcNAcb1-2Mana-Sp0 & 17 & 13 & 75 \\
\hline 250 & Neu5Aca2-3Galb1-3GIcNAcb-Sp0 & 17 & 19 & 115 \\
\hline 97 & GalNAcb1-3Gala1-4Galb1-4GIcNAcb-Sp0 & 17 & 4 & 24 \\
\hline 113 & Gala1-3GalNAcb-Sp8 & 17 & 12 & 73 \\
\hline 332 & Neu5Aca2-3Galb1-3(Fuca1-4)GlcNAcb1-3Galb1-3(Fuca1-4)GIcNAcb-Sp0 & 17 & 12 & 70 \\
\hline 412 & Galb1-3GalNAcb1-4(Neu5Aca2-8Neu5Aca2-3)Galb1-4Glcb-Sp0 & 17 & 7 & 44 \\
\hline 129 & Galb1-3(Fuca1-4)GlcNAc-Sp0 & 17 & 10 & 60 \\
\hline 237 & Neu5Aca2-3GalNAca-Sp8 & 17 & 7 & 45 \\
\hline 331 & Neu5Aca2-6Galb1-4GIcNAcb1-3Galb1-3GIcNAcb-Sp0 & 16 & 3 & 19 \\
\hline 520 & GalNAca1-3(Fuca1-2)Galb1-4GIcNAcb1-6GalNAc-Sp14 & 16 & 9 & 55 \\
\hline 274 & Neu5Aca2-6Galb1-4GIcb-Sp8 & 16 & 4 & 26 \\
\hline 358 & KDNa2-6Galb1-4GıcNAc-Sp0 & 16 & 12 & 75 \\
\hline 213 & Mana1-2Mana1-6(Mana1-2Mana1-3)Mana1-6(Mana1-2Mana1-2Mana1-3)Manb1-4GIcNAcb1-4GIcNAcb-Sp12 & 16 & 12 & 75 \\
\hline 543 & $\begin{array}{l}\text { Galb1-4GlcNAcb1-3Galb1-4GIcNAcb1-2Mana1-6(Galb1-4GlcNAcb1-3Galb1-4GlcNAcb1-2Mana1-3)Manb1- } \\
\text { 4GIcNAcb1-4GlcNAcb-Sp24 }\end{array}$ & 16 & 4 & 22 \\
\hline 15 & GalNAcb-Sp8 & 16 & 12 & 73 \\
\hline 228 & Neu5Aca2-8Neu5Aca2-8Neu5Aca2-3Galb1-4Glcb-Sp0 & 16 & 8 & 51 \\
\hline 488 & Neu5Aca2-6Galb1-4GIcNAcb1-6(Fuca1-2Galb1-4(Fuca1-3)GlcNAcb1-3)Galb1-4GIc-Sp21 & 16 & 5 & 29 \\
\hline 131 & Fuca1-4(Galb1-3)GlcNAcb-Sp8 & 16 & 8 & 49 \\
\hline
\end{tabular}




\begin{tabular}{|c|c|c|c|c|}
\hline 439 & $\begin{array}{l}\text { Galb1-4GIcNAcb1-6(Galb1-4GIcNAcb1-2)Mana1-6(GIcNAcb1-4)(Galb1-4GIcNAcb1-4(Galb1-4GlcNAcb1- } \\
\text { 2)Mana1-3)Manb1-4GIcNAcb1-4GIcNAc-Sp21 }\end{array}$ & 16 & 5 & 33 \\
\hline 57 & $\begin{array}{l}\text { Neu5Aca2-6Galb1-4GIcNAcb1-2Mana1-6(Neu5Aca2-6Galb1-4GlcNAcb1-2Man-a1-3)Manb1-4GlcNAcb1- } \\
\text { 4GIcNAcb-Sp21 }\end{array}$ & 16 & 9 & 59 \\
\hline 270 & Neu5Aca2-6Galb1-4GIcNAcb-Sp8 & 16 & 5 & 34 \\
\hline 539 & Galb1-3GalNAcb1-3Gal-Sp21 & 16 & 2 & 14 \\
\hline 22 & 6S(3S)Galb1-4(6S)GIcNAcb-Sp0 & 16 & 16 & 104 \\
\hline 69 & Fuca1-2Galb1-3GIcNAcb-Sp8 & 16 & 10 & 66 \\
\hline 384 & Galb1-4(Fuca1-3)GlcNAcb1-6(Galb1-3GlcNAcb1-3)Galb1-4Glc-Sp21 & 16 & 16 & 102 \\
\hline 144 & Galb1-3GalNAcb1-3Gala1-4Galb1-4Glcb-Sp0 & 16 & 8 & 49 \\
\hline 253 & Neu5Aca2-3Galb1-4(Fuca1-3)(6S)GlcNAcb-Sp8 & 16 & 9 & 57 \\
\hline 602 & Neu5Aca2-6Galb1-4GIcNAcb1-3Galb1-4GIcNAcb1-6(Galb1-3)GalNAca-Sp14 & 15 & 4 & 27 \\
\hline 599 & GlcNAcb1-3Galb1-4GlcNAcb1-3Galb1-4GlcNAcb1-3GalNAca-Sp14 & 15 & 7 & 44 \\
\hline 264 & Neu5Aca2-3Galb1-4GIcb-Sp0 & 15 & 7 & 43 \\
\hline 179 & GlcNAcb1-6(GIcNAcb1-3)Galb1-4GlcNAcb-Sp8 & 15 & 5 & 32 \\
\hline 481 & Neu5Aca2-6Galb1-4GIcNAcb1-6GalNAca-Sp14 & 15 & 8 & 50 \\
\hline 478 & Neu5Aca2-6Galb1-4GIcNAcb1-6(Galb1-3GlcNAcb1-3)Galb1-4Glcb-Sp21 & 15 & 7 & 47 \\
\hline 588 & $\begin{array}{l}\text { GlcNAcb1-3Galb1-4GlcNAcb1-3Galb1-4GlcNAcb1-3Galb1-4GlcNAcb1-3Galb1-4GlcNAcb1-6(GlcNAcb1-3Galb1- } \\
\text { 4GlcNAcb1-3Galb1-4GIcNAcb1-3Galb1-4GlcNAcb1-3Galb1-4GlcNAb1-2)Mana1-6(GlcNAcb1-3Galb1- } \\
\text { 4GlcNAcb1-3Galb1-4GlcNAcb1-3Galb1-4GlcNAcb1-3Galb1-4GlcNAcb1-2Mana1-3)Manb1-4GlcNAcb1-4(Fuca1- } \\
\text { 6)GlcNAcb-Sp24 }\end{array}$ & 15 & 4 & 26 \\
\hline 309 & Neu5Aca2-6Galb1-4GIcNAcb1-2Mana1-6(GlcNAcb1-2Mana1-3)Manb1-4GlcNAcb1-4GlcNAcb-Sp12 & 15 & 17 & 116 \\
\hline 596 & GlcNAcb1-3Galb1-4GIcNAcb1-6(GlcNAcb1-3Galb1-4GIcNAcb1-3)GalNAca-Sp14 & 15 & 11 & 73 \\
\hline 357 & KDNa2-3Galb1-4(Fuca1-3)GIcNAc-Sp0 & 15 & 10 & 69 \\
\hline 360 & KDNa2-3Galb1-3GalNAca-Sp14 & 15 & 11 & 74 \\
\hline 52 & GlcNAcb1-2Mana1-6(GlcNAcb1-2Mana1-3)Manb1-4GlcNAcb1-4GIcNAcb-Sp12 & 15 & 8 & 53 \\
\hline 590 & Galb1-4GIcNAcb1-3Galb1-4GIcNAcb1-3GalNAca-Sp14 & 15 & 5 & 34 \\
\hline 149 & Galb1-3GlcNAcb1-3Galb1-4Glcb-Sp10 & 15 & 5 & 34 \\
\hline 232 & GalNAcb1-4(Neu5Aca2-3)Galb1-4GIcNAcb-Sp0 & 15 & 10 & 68 \\
\hline 444 & (6S)Galb1-3GlcNAcb-Sp0 & 15 & 10 & 69 \\
\hline 432 & GlcNAcb1-2Mana1-6(GlcNAcb1-4)(GlcNAcb1-2Mana1-3)Manb1-4GlcNAcb1-4GIcNAc-Sp21 & 15 & 9 & 58 \\
\hline 123 & Gala1-4Galb1-4GIcb-Sp0 & 15 & 15 & 103 \\
\hline 382 & Galb1-3GalNAca1-3(Fuca1-2)Galb1-4GIcNAc-Sp0 & 15 & 8 & 55 \\
\hline 134 & GlcNAcb1-6(Galb1-3)GalNAca-Sp8 & 15 & 11 & 75 \\
\hline 458 & $\begin{array}{l}\text { GalNAca1-3(Fuca1-2)Galb1-3GIcNAcb1-2Mana1-6(GalNAca1-3(Fuca1-2)Galb1-3GlcNAcb1-2Mana1-3)Manb1- } \\
\text { 4GlcNAcb1-4(Fuca1-6)GIcNAcb-Sp22 }\end{array}$ & 15 & 9 & 59 \\
\hline 43 & (6S)Galb1-4Glcb-Sp8 & 15 & 5 & 35 \\
\hline 551 & $\begin{array}{l}\text { Galb1-4GIcNAcb1-3Galb1-4GlcNAcb1-3Galb1-4GlcNAcb1-3Galb1-4GIcNAcb1-2Mana1-6(Galb1-4GIcNAcb1- } \\
\text { 3Galb1-4GIcNAcb1-3Galb1-4GlcNAcb1-3Galb1-4GIcNAcb1-2Mana1-3)Manb1-4GIcNAcb1-4GlcNAcb-Sp25 }\end{array}$ & 15 & 4 & 25 \\
\hline 504 & GalNAcb1-4(Fuca1-3)(6S)GlcNAcb-Sp8 & 15 & 18 & 121 \\
\hline 528 & Gala1-3Galb1-3GlcNAcb1-2Mana-Sp0 & 15 & 8 & 58 \\
\hline 282 & Neu5Gca2-3Galb1-3GIcNAcb-Sp0 & 14 & 6 & 40 \\
\hline 468 & Gala1-3(Fuca1-2)Galb1-3GalNAca-Sp8 & 14 & 8 & 58 \\
\hline
\end{tabular}




\begin{tabular}{|c|c|c|c|c|}
\hline 152 & Galb1-4(Fuca1-3)GlcNAcb-Sp0 & 14 & 2 & 12 \\
\hline 317 & Mana1-2Mana1-6(Mana1-2Mana1-3)Mana1-6(Mana1-2Mana1-2Mana1-3)Mana-Sp9 & 14 & 13 & 87 \\
\hline 66 & Fuca1-2Galb1-3GIcNAcb1-3Galb1-4GIcb-Sp8 & 14 & 5 & 35 \\
\hline 125 & Gala1-6Glcb-Sp8 & 14 & 4 & 29 \\
\hline 492 & Neu5Aca2-3Galb1-3GIcNAcb1-6GalNAca-Sp14 & 14 & 8 & 57 \\
\hline 493 & (3S)Galb1-3(Fuca1-4)GIcNAcb-Sp0 & 14 & 4 & 30 \\
\hline 471 & Glca1-4Glca1-4GIca1-4GIcb-Sp10 & 14 & 9 & 67 \\
\hline 295 & Neu5Aca2-3Galb1-3GIcNAcb1-3Galb1-3GIcNAcb-Sp0 & 14 & 2 & 16 \\
\hline 219 & Neu5Aca2-3Galb1-4GlcNAcb1-3Galb1-4(Fuca1-3)GIcNAcb-Sp0 & 14 & 13 & 92 \\
\hline 146 & Galb1-3GalNAcb1-4Galb1-4Glcb-Sp8 & 14 & 7 & 50 \\
\hline 305 & Galb1-4GlcNAca1-6Galb1-4GIcNAcb-Sp0 & 14 & 8 & 57 \\
\hline 159 & Galb1-4GalNAcb1-3(Fuca1-2)Galb1-4GlcNAcb-Sp8 & 14 & 9 & 65 \\
\hline 100 & GalNAcb1-4GIcNAcb-Sp8 & 14 & 2 & 13 \\
\hline 380 & GalNAcb1-4GIcNAcb1-2Mana1-6(GalNAcb1-4GIcNAcb1-2Mana1-3)Manb1-4GIcNAcb1-4GIcNAc-Sp12 & 14 & 10 & 69 \\
\hline 459 & $\begin{array}{l}\text { Galb1-4GIcNAcb1-6(Galb1-4GIcNAcb1-2)Mana1-6(Galb1-4GIcNAcb1-2Mana1-3)Manb1-4GIcNAcb1-4GIcNAcb- } \\
\text { Sp19 }\end{array}$ & 14 & 4 & 26 \\
\hline 13 & Glcb-Sp8 & 14 & 12 & 84 \\
\hline 160 & Galb1-4GlcNAcb1-3GalNAca-Sp8 & 14 & 12 & 83 \\
\hline 7 & Fuca-Sp9 & 14 & 2 & 16 \\
\hline 173 & Galb1-4Glcb-Sp8 & 14 & 11 & 80 \\
\hline 355 & Galb1-3GIcNAcb1-2Mana1-6(Galb1-3GIcNAcb1-2Mana1-3)Manb1-4GIcNAcb1-4(Fuca1-6)GIcNAcb-Sp22 & 14 & 20 & 144 \\
\hline 38 & (3S)Galb-Sp8 & 14 & 3 & 20 \\
\hline 333 & Neu5Aca2-6Galb1-4GIcNAcb1-3Galb1-4GIcNAcb1-3Galb1-4GIcNAcb-Sp0 & 14 & 7 & 52 \\
\hline 377 & Neu5Aca2-3Galb1-4GIcNAcb1-3GalNAc-Sp14 & 14 & 4 & 26 \\
\hline 44 & (6S)Galb1-4GlcNAcb-Sp8 & 14 & 10 & 70 \\
\hline 216 & Mana1-6(Mana1-3)Mana1-6(Mana1-2Mana1-3)Manb1-4GlcNAcb1-4GlcNAcb-Sp12 & 14 & 7 & 48 \\
\hline 147 & Galb1-3Galb-Sp8 & 14 & 5 & 34 \\
\hline 48 & $\mathrm{Neu} 5,9 \mathrm{Ac}_{2} \mathrm{a}-\mathrm{Sp} 8$ & 14 & 12 & 90 \\
\hline 446 & $\begin{array}{l}\text { Fuca1-2Galb1-4 GlcNAcb1-2Mana1-6(Fuca1-2Galb1-4GlcNAcb1-2(Fuca1-2Galb1-4GIcNAcb1-4)Mana1- } \\
\text { 3)Manb1-4GIcNAcb1-4GIcNAcb-Sp12 }\end{array}$ & 14 & 7 & 54 \\
\hline 67 & Fuca1-2Galb1-3GlcNAcb1-3Galb1-4GIcb-Sp10 & 14 & 7 & 50 \\
\hline 189 & GlcNAcb1-4Galb1-4GlcNAcb-Sp8 & 13 & 6 & 45 \\
\hline 514 & (3S)GalNAcb1-4(3S)GlcNAc-Sp8 & 13 & 10 & 77 \\
\hline 413 & Neu5Aca2-3Galb1-3GalNAcb1-4(Neu5Aca2-8Neu5Aca2-3)Galb1-4Glcb-Sp0 & 13 & 9 & 66 \\
\hline 392 & Galb1-3GIcNAcb1-3GalNAca-Sp14 & 13 & 4 & 27 \\
\hline 16 & GlcNAcb-Sp0 & 13 & 3 & 26 \\
\hline 452 & GalNAca1-3(Fuca1-2)Galb1-4GIcNAcb1-6(GalNAca1-3(Fuca1-2)Galb1-4GlcNAcb1-3)GalNAc-Sp14 & 13 & 13 & 96 \\
\hline 193 & GlcNAcb1-6GalNAca-Sp8 & 13 & 15 & 116 \\
\hline 240 & Neu5Aca2-3Galb1-3(Fuca1-4)GlcNAcb-Sp8 & 13 & 14 & 109 \\
\hline 526 & Gala1-3(Fuca1-2)Galb1-3GlcNAcb1-6GalNAc-Sp14 & 13 & 4 & 33 \\
\hline 552 & $\begin{array}{l}\text { Galb1-3GIcNAcb1-3Galb1-4GIcNAcb1-2Mana1-6(Galb1-3GlcNAcb1-3Galb1-4GIcNAcb1-2Mana1-3)Manb1- } \\
\text { 4GlcNAcb1-4GIcNAc-Sp25 }\end{array}$ & 13 & 10 & 73 \\
\hline
\end{tabular}




\begin{tabular}{|c|c|c|c|c|}
\hline 467 & $\begin{array}{l}\text { Neu5Aca2-6Galb1-4GlcNAcb1-6(Neu5Aca2-6Galb1-4GlcNAcb1-2)Mana1-6(GlcNAcb1-4)(Neu5Aca2-6Galb1- } \\
\text { 4GIcNAcb1-4(Neu5Aca2-6Galb1-4GlcNAcb1-2)Mana1-3)Manb1-4GlcNAcb1-4GlcNAcb-Sp21 }\end{array}$ & 13 & 5 & 36 \\
\hline 86 & GalNAca1-3(Fuca1-2)Galb1-4GIcNAcb-Sp0 & 13 & 4 & 34 \\
\hline 153 & Galb1-4(Fuca1-3)GIcNAcb-Sp8 & 13 & 7 & 56 \\
\hline 438 & $\begin{array}{l}\text { Galb1-4GIcNAcb1-6(Galb1-4GIcNAcb1-2)Mana1-6(GIcNAcb1-4)(Galb1-4GIcNAcb1-2Mana1-3)Manb1- } \\
\text { 4GIcNAcb1-4GIcNAc-Sp21 }\end{array}$ & 13 & 7 & 51 \\
\hline 453 & Neu5Aca2-8Neu5Aca2-3Galb1-3GalNAcb1-4(Neu5Aca2-8Neu5Aca2-3)Galb1-4GIcb-Sp0 & 13 & 13 & 101 \\
\hline 320 & Galb1-4GIcNAcb1-2Mana1-6(Neu5Aca2-6Galb1-4GIcNAcb1-2Mana1-3)Manb1-4GIcNAcb1-4GIcNAcb-Sp12 & 13 & 6 & 44 \\
\hline 3 & Mana-Sp8 & 13 & 9 & 67 \\
\hline 434 & GlcNAcb1-6(GlcNAcb1-2)Mana1-6(GlcNAcb1-4)(GlcNAcb1-2Mana1-3)Manb1-4GlcNAcb1-4GlcNAc-Sp21 & 13 & 4 & 32 \\
\hline 476 & GlcNAcb1-6(GlcNAcb1-2)Mana1-6(GIcNAcb1-2Mana1-3)Manb1-4GIcNAcb1-4(Fuca1-6)GlcNAcb-Sp24 & 13 & 12 & 94 \\
\hline 424 & Gala1-3(Fuca1-2)Galb1-3GIcNAcb1-3GalNAc-Sp14 & 13 & 8 & 64 \\
\hline 436 & Galb1-4GIcNAcb1-2Mana1-6(GlcNAcb1-4)(Galb1-4GIcNAcb1-2Mana1-3)Manb1-4GlcNAcb1-4GIcNAc-Sp21 & 13 & 6 & 48 \\
\hline 289 & Neu5Aca2-3Galb1-4GIcNAcb1-6(Galb1-3)GalNAca-Sp14 & 13 & 7 & 58 \\
\hline 359 & KDNa2-3Galb1-4Glc-Sp0 & 13 & 6 & 47 \\
\hline 241 & Neu5Aca2-3Galb1-3(Fuca1-4)GlcNAcb1-3Galb1-4(Fuca1-3)GIcNAcb-Sp0 & 13 & 4 & 30 \\
\hline 499 & Gala1-3(Fuca1-2)Galb1-4GIcNAcb1-6GalNAca-Sp14 & 13 & 7 & 53 \\
\hline 390 & Fuca1-2Galb1-3GalNAca1-3(Fuca1-2)Galb1-4Glcb-Sp0 & 13 & 8 & 62 \\
\hline 505 & (3S)GalNAcb1-4(Fuca1-3)GlcNAcb-Sp8 & 13 & 4 & 34 \\
\hline 418 & Gala1-3(Fuca1-2)Galb1-4(Fuca1-3)GlcNAcb1-3GalNAc-Sp14 & 13 & 3 & 23 \\
\hline 303 & Galb1-4GIcNAcb1-6(Galb1-4GIcNAcb1-3)Galb1-4GlcNAc-Sp0 & 13 & 4 & 34 \\
\hline 6 & Fuca-Sp8 & 13 & 1 & 7 \\
\hline 230 & Neu5Aca2-8Neu5Aca2-8Neu5Aca-Sp8 & 13 & 4 & 33 \\
\hline 498 & Fuca1-2(6S)Galb1-3GıcNAcb-Sp0 & 13 & 6 & 50 \\
\hline 324 & Neu5Gcb2-6Galb1-4GIcNAc-Sp8 & 13 & 6 & 52 \\
\hline 307 & GalNAcb1-3Galb-Sp8 & 13 & 3 & 26 \\
\hline 402 & Galb1-4(Fuca1-3)GlcNAcb1-3GalNAca-Sp14 & 12 & 6 & 44 \\
\hline 365 & Galb1-4GIcNAcb1-2Mana1-6(Mana1-3)Manb1-4GIcNAcb1-4GIcNAcb-Sp12 & 12 & 6 & 50 \\
\hline 388 & $\begin{array}{l}\text { Galb1-4GIcNAcb1-6(Galb1-4GIcNAcb1-2)Mana1-6(Galb1-4GIcNAcb1-4(Galb1-4GIcNAcb1-2)Mana1-3)Manb1- } \\
\text { 4GIcNAcb1-4GIcNAcb-Sp21 }\end{array}$ & 12 & 5 & 37 \\
\hline 422 & GlcNAcb1-2(GlcNAcb1-6)Mana1-6(GlcNAcb1-2Mana1-3)Manb1-4GlcNAcb1-4GIcNAcb-Sp19 & 12 & 4 & 32 \\
\hline 349 & Neu5Aca2-6Galb1-4GIcNAcb1-2Mana1-3Manb1-4GIcNAcb1-4GIcNAc-Sp12 & 12 & 10 & 77 \\
\hline 199 & Glcb1-4Glcb-Sp8 & 12 & 9 & 75 \\
\hline 600 & Galb1-4GlcNAcb1-3Galb1-3GalNAca-Sp14 & 12 & 2 & 19 \\
\hline 61 & Fuca1-2Galb1-3(Fuca1-4)GıNAcb-Sp8 & 12 & 9 & 74 \\
\hline 531 & GlcNAcb1-2 Mana1-6(GlcNAcb1-4)(GlcNAcb1-2Mana1-3)Manb1-4GlcNAcb1-4(Fuca1-6)GIcNAc-Sp21 & 12 & 8 & 63 \\
\hline 373 & $\begin{array}{l}\text { Gala1-3Galb1-4(Fuca1-3)GlcNAcb1-2Mana1-6(Gala1-3Galb1-4(Fuca1-3)GlcNAcb1-2Mana1-3)Manb1- } \\
\text { 4GlcNAcb1-4GlcNAcb-Sp20 }\end{array}$ & 12 & 4 & 34 \\
\hline 536 & GIcNAcb1-3Galb1-4GIcNAcb1-6(GIcNAcb1-3)Galb1-4GIcNAc-Sp0 & 12 & 10 & 80 \\
\hline 184 & GlcNAcb1-3Galb1-4GlcNAcb-Sp8 & 12 & 12 & 101 \\
\hline 222 & Fuca1-2Galb1-4(6S)GlcNAcb-Sp8 & 12 & 8 & 63 \\
\hline 49 & Neu5,9Ac2a2-6Galb1-4GlcNAcb-Sp8 & 12 & 9 & 76 \\
\hline 361 & Fuca1-2Galb1-3GIcNAcb1-2Mana1-6(Fuca1-2Galb1-3GIcNAcb1-2Mana1-3)Manb1-4GIcNAcb1-4GIcNAcb-Sp20 & 12 & 6 & 50 \\
\hline
\end{tabular}




\begin{tabular}{|c|c|c|c|c|}
\hline 369 & Galb1-4(Fuca1-3)GlcNAcb1-6(Fuca1-2Galb1-4GIcNAcb1-3)Galb1-4GIc-Sp21 & 12 & 11 & 97 \\
\hline 95 & GalNAcb1-3GalNAca-Sp8 & 12 & 7 & 60 \\
\hline 234 & GalNAcb1-4(Neu5Aca2-3)Galb1-4Glcb-Sp0 & 12 & 5 & 45 \\
\hline 466 & $\begin{array}{l}\text { Neu5Aca2-6Galb1-4GIcNAcb1-6(Neu5Aca2-6Galb1-4GlcNAcb1-2)Mana1-6(GlcNAcb1-4)(Neu5Aca2-6Galb1- } \\
\text { 4GlcNAcb1-2Mana1-3)Manb1-4GlcNAcb1-4GIcNAcb-Sp21 }\end{array}$ & 12 & 8 & 69 \\
\hline 416 & GalNAca1-3GalNAcb1-3Gala1-4Galb1-4Glcb-Sp0 & 12 & 4 & 35 \\
\hline 344 & GIcNAca1-4Galb1-4GIcNAcb1-3Galb1-4GlcNAcb-Sp0 & 12 & 8 & 67 \\
\hline 352 & Mana1-6(Galb1-4GIcNAcb1-2Mana1-3)Manb1-4GIcNAcb1-4GIcNAcb-Sp12 & 12 & 4 & 38 \\
\hline 94 & GalNAca1-4(Fuca1-2)Galb1-4GIcNAcb-Sp8 & 12 & 7 & 61 \\
\hline 260 & Neu5Aca2-3Galb1-4GIcNAcb-Sp0 & 11 & 3 & 27 \\
\hline 316 & Mana1-2Mana1-6(Mana1-3)Mana1-6(Mana1-2Mana1-2Mana1-3)Mana-Sp9 & 11 & 10 & 85 \\
\hline 80 & Fuca1-3GIcNAcb-Sp8 & 11 & 9 & 81 \\
\hline 64 & Fuca1-2Galb1-3GalNAcb1-4(Neu5Aca2-3)Galb1-4Glcb-Sp0 & 11 & 3 & 26 \\
\hline 393 & GalNAcb1-4(Neu5Aca2-3)Galb1-4GIcNAcb1-3GalNAca-Sp14 & 11 & 7 & 60 \\
\hline 497 & Galb1-4(Fuca1-3)GIcNAcb1-2Mana-Sp0 & 11 & 1 & 7 \\
\hline 19 & Galb1-4GIcNAcb1-6(Galb1-4GlcNAcb1-3)GalNAca-Sp8 & 11 & 6 & 52 \\
\hline 511 & Galb1-3(6S)GlcNAcb-Sp8 & 11 & 6 & 49 \\
\hline 54 & Galb1-4GIcNAcb1-2Mana1-6(Galb1-4GIcNAcb1-2Mana1-3)Manb1-4GIcNAcb1-4GIcNAcb-Sp12 & 11 & 3 & 27 \\
\hline 208 & Mana1-2Mana1-2Mana1-3Mana-Sp9 & 11 & 6 & 51 \\
\hline 138 & Neu5Acb2-6(Galb1-3)GalNAca-Sp8 & 11 & 8 & 73 \\
\hline 545 & $\begin{array}{l}\text { Fuca1-2Galb1-4GIcNAcb1-3Galb1-4GlcNAcb1-2Mana1-6(Fuca1-2Galb1-4GlcNAcb1-3Galb1-4GlcNAcb1- } \\
\text { 2Mana1-3)Manb1-4GlcNAcb1-4GlcNAcb-Sp24 }\end{array}$ & 11 & 5 & 48 \\
\hline 480 & Neu5Aca2-3Galb1-4GIcNAcb1-6GalNAca-Sp14 & 11 & 10 & 90 \\
\hline 194 & GlcNAcb1-6GalNAca-Sp14 & 11 & 6 & 53 \\
\hline 105 & Gala1-3(Fuca1-2)Galb1-4(Fuca1-3)GlcNAcb-Sp8 & 11 & 5 & 44 \\
\hline 50 & Mana1-6(Mana1-3)Manb1-4GlcNAcb1-4GlcNAcb-Sp12 & 11 & 3 & 29 \\
\hline 220 & (3S)Galb1-4(Fuca1-3)(6S)GlcNAcb-Sp8 & 11 & 4 & 35 \\
\hline 513 & (6S)GalNAcb1-4GIcNAc-Sp8 & 11 & 3 & 25 \\
\hline 23 & $6 \mathrm{~S}(3 \mathrm{~S}) \mathrm{Galb1} 1-4 \mathrm{GlcNAcb}-\mathrm{Sp} 0$ & 11 & 6 & 57 \\
\hline 400 & Neu5Aca2-3Galb1-3GIcNAcb1-3GalNAca-Sp14 & 11 & 3 & 28 \\
\hline 474 & $\begin{array}{l}\text { Fuca1-2Galb1-3(Fuca1-4)GIcNAcb1-2Mana1-6(Fuca1-2Galb1-3(Fuca1-4)GIcNAcb1-2Mana1-3)Manb1- } \\
\text { 4GIcNAcb1-4(Fuca1-6)GIcNAcb1-4(Fuca1-6)GIcNAcb-Sp19 }\end{array}$ & 11 & 4 & 37 \\
\hline 383 & Galb1-3GlcNAcb1-3Galb1-4GIcNAcb1-6(Galb1-3GlcNAcb1-3)Galb1-4GIcb-Sp0 & 11 & 5 & 46 \\
\hline 130 & Galb1-3(Fuca1-4)GlcNAc-Sp8 & 11 & 5 & 49 \\
\hline 198 & Glca1-6Glca1-6Glcb-Sp8 & 11 & 7 & 63 \\
\hline 252 & Neu5Aca2-3Galb1-4(6S)GlcNAcb-Sp8 & 11 & 4 & 33 \\
\hline 246 & Neu5Aca2-3Galb-Sp8 & 11 & 22 & 203 \\
\hline 46 & Neu5Aca2-3(6S)Galb1-4GlcNAcb-Sp8 & 11 & 14 & 130 \\
\hline
\end{tabular}




\begin{tabular}{|c|c|c|c|c|}
\hline 45 & (6S)Galb1-4(6S)GIcb-Sp8 & 11 & 10 & 90 \\
\hline 547 & $\begin{array}{l}\text { GlcNAcb1-3Galb1-4GlcNAcb1-3Galb1-4GlcNAcb1-2Mana1-6(GlcNAcb1-3Galb1-4GlcNAcb1-3Galb1-4GlcNAcb1- } \\
\text { 2Mana1-3)Manb1-4GlcNAcb1-4GlcNAcb-Sp25 }\end{array}$ & 11 & 4 & 37 \\
\hline 387 & Galb1-3GlcNAcb1-3Galb1-4(Fuca1-3)GlcNAcb1-6(Galb1-3GlcNAcb1-3)Galb1-4Glc-Sp21 & 11 & 5 & 46 \\
\hline 257 & Neu5Aca2-3Galb1-4(Fuca1-3)GIcNAcb1-3Galb-Sp8 & 11 & 7 & 61 \\
\hline 398 & GlcNAcb1-2Mana1-6(Galb1-4GIcNAcb1-2Mana1-3)Manb1-4GlcNAcb1-4GIcNAc-Sp12 & 11 & 10 & 92 \\
\hline 473 & $\begin{array}{l}\text { Fuca1-2Galb1-4(Fuca1-3)GIcNAcb1-2Mana1-6(Fuca1-2Galb1-4(Fuca1-3)GlcNAcb1-2Mana1-3)Manb1- } \\
\text { 4GIcNAcb1-4(Fuca1-6)GIcNAcb-Sp24 }\end{array}$ & 11 & 7 & 61 \\
\hline 201 & G-ol-Sp8 & 11 & 4 & 36 \\
\hline 209 & Mana1-2Mana1-6(Mana1-2Mana1-3)Mana-Sp9 & 11 & 2 & 20 \\
\hline 68 & Fuca1-2Galb1-3GlcNAcb-Sp0 & 11 & 14 & 130 \\
\hline 217 & Mana1-6(Mana1-3)Mana1-6(Mana1-3)Manb1-4GIcNAcb1-4GIcNAcb-Sp12 & 11 & 2 & 22 \\
\hline 166 & Galb1-4GlcNAcb1-3Galb1-4Glcb-Sp8 & 11 & 12 & 111 \\
\hline 470 & Glca1-6Glca1-6Glca1-6Glcb-Sp10 & 11 & 3 & 30 \\
\hline 51 & Mana1-6(Mana1-3)Manb1-4GIcNAcb1-4GIcNAcb-Sp13 & 11 & 3 & 28 \\
\hline 28 & (3S)Galb1-3(Fuca1-4)GIcNAcb-Sp8 & 11 & 5 & 43 \\
\hline 483 & $\begin{array}{l}\text { Neu5Aca2-6Galb1-4GlcNAcb1-2Mana1-6(Neu5Aca2-6Galb1-4GlcNAcb1-2Mana1-3)Manb1-4GlcNAcb1-4(Fuca1- } \\
\text { 6)GlcNAcb-Sp24 }\end{array}$ & 10 & 3 & 29 \\
\hline 512 & $(6 \mathrm{~S})(4 \mathrm{~S}) \mathrm{GalNAcb} 1-4 \mathrm{GlcNAc-Sp8}$ & 10 & 10 & 92 \\
\hline 594 & GlcNAcb1-3Galb1-4GIcNAcb1-3GalNAca-Sp14 & 10 & 2 & 18 \\
\hline 175 & GIcNAca1-6Galb1-4GIcNAcb-Sp8 & 10 & 7 & 69 \\
\hline 279 & Neu5Acb2-6GalNAca-Sp8 & 10 & 10 & 96 \\
\hline 215 & Mana1-2Mana1-2Mana1-6(Mana1-3)Mana-Sp9 & 10 & 5 & 53 \\
\hline 550 & $\begin{array}{l}\text { GlcNAcb1-3Galb1-4GlcNAcb1-3Galb1-4GlcNAcb1-3Galb1-4GlcNAcb1-2Mana1-6(GlcNAcb1-3Galb1-4GIcNAcb1- } \\
\text { 3Galb1-4GlcNAcb1-3Galb1-4GIcNAcb1-2Mana1-3)Manb1-4GIcNAcb1-4GIcNAcb-Sp25 }\end{array}$ & 10 & 9 & 87 \\
\hline 370 & $\begin{array}{l}\text { Galb1-4GlcNAcb1-2Mana1-6(Galb1-4GlcNAcb1-4(Galb1-4GlcNAcb1-2)Mana1-3)Manb1-4GIcNAcb1-4GIcNAc- } \\
\text { Sp21 }\end{array}$ & 10 & 8 & 82 \\
\hline 151 & Galb1-3GlcNAcb-Sp8 & 10 & 4 & 41 \\
\hline 506 & Fuca1-2Galb1-3GIcNAcb1-6(Fuca1-2Galb1-3GIcNAcb1-3)GalNAca-Sp14 & 10 & 2 & 23 \\
\hline 154 & Galb1-4(Fuca1-3)GlcNAcb1-3Galb1-4(Fuca1-3)GlcNAcb-Sp0 & 10 & 6 & 59 \\
\hline 517 & (4S)GalNAcb-Sp10 & 10 & 10 & 94 \\
\hline 205 & GlcAb1-6Galb-Sp8 & 10 & 10 & 99 \\
\hline 182 & GlcNAcb1-3Galb-Sp8 & 10 & 7 & 71 \\
\hline 238 & Neu5Aca2-3GalNAcb1-4GlcNAcb-Sp0 & 10 & 11 & 113 \\
\hline 293 & Galb1-4(Fuca1-3)GlcNAcb1-3Galb1-3(Fuca1-4)GlcNAcb-Sp0 & 10 & 9 & 87 \\
\hline 409 & GalNAcb1-3Gala1-6Galb1-4Glcb-Sp8 & 10 & 6 & 56 \\
\hline 83 & GalNAca1-3(Fuca1-2)Galb1-3GIcNAcb-Sp0 & 10 & 17 & 167 \\
\hline 225 & Neu5Aca2-3Galb1-3GalNAca-Sp14 & 10 & 4 & 44 \\
\hline 306 & Galb1-4GIcNAcb1-6Galb1-4GIcNAcb-Sp0 & 10 & 4 & 36 \\
\hline 586 & $\begin{array}{l}\text { GlcNAcb1-3Galb1-4GlcNAcb1-3Galb1-4GIcNAcb1-3Galb1-4GlcNAcb1-6(GlcNAcb1-3Galb1-4GlcNAcb1-3Galb1- } \\
\text { 4GlcNAcb1-3Galb1-4GIcNAb1-2)Mana1-6(GlcNAcb1-3Galb1-4GlcNAcb1-3Galb1-4GlcNAcb1-3Galb1- } \\
\text { 4GlcNAcb1-2Mana1-3)Manb1-4GlcNAcb1-4(Fuca1-6)GlcNAcb-Sp24 }\end{array}$ & 10 & 3 & 33 \\
\hline
\end{tabular}




\begin{tabular}{|c|c|c|c|c|}
\hline 63 & Fuca1-2Galb1-3GalNAca-Sp14 & 10 & 6 & 59 \\
\hline 212 & Mana1-2Mana1-6(Mana1-3)Mana1-6(Mana1-2Mana1-2Mana1-3)Manb1-4GIcNAcb1-4GIcNAcb-Sp12 & 10 & 9 & 89 \\
\hline 165 & Galb1-4GlcNAcb1-3Galb1-4Glcb-Sp0 & 10 & 3 & 29 \\
\hline 71 & Fuca1-2Galb1-4(Fuca1-3)GIcNAcb1-3Galb1-4(Fuca1-3)GlcNAcb1-3Galb1-4(Fuca1-3)GIcNAcb-Sp0 & 10 & 7 & 69 \\
\hline 299 & (6P)Glcb-Sp10 & 10 & 4 & 40 \\
\hline 495 & Fuca1-2Galb1-4GlcNAcb1-6GalNAca-Sp14 & 10 & 4 & 41 \\
\hline 132 & Galb1-4GIcNAcb1-6GalNAca-Sp8 & 10 & 7 & 72 \\
\hline 231 & Neu5Aca2-3(6S)Galb1-4(Fuca1-3)GlcNAcb-Sp8 & 10 & 3 & 28 \\
\hline 91 & GalNAca1-3(Fuca1-2)Galb-Sp18 & 10 & 7 & 72 \\
\hline 312 & GlcNAcb1-4GIcNAcb-Sp12 & 10 & 14 & 142 \\
\hline 290 & Galb1-3GIcNAcb1-3Galb1-3GIcNAcb-Sp0 & 10 & 3 & 29 \\
\hline 389 & GlcNAcb1-2Mana1-6(GlcNAcb1-4(GlcNAcb1-2)Mana1-3)Manb1-4GIcNAcb1-4GIcNAc-Sp21 & 10 & 4 & 43 \\
\hline 287 & Neu5Gca2-6Galb1-4GlcNAcb-Sp0 & 10 & 12 & 130 \\
\hline 255 & Neu5Aca2-3Galb1-4(Fuca1-3)GIcNAcb-Sp0 & 10 & 4 & 38 \\
\hline 187 & GlcNAcb1-4-MDPLys & 10 & 7 & 70 \\
\hline 137 & Neu5Aca2-6(Galb1-3)GalNAca-Sp14 & 10 & 4 & 47 \\
\hline 157 & Galb1-4(6S)Glcb-Sp8 & 10 & 13 & 141 \\
\hline 65 & Fuca1-2Galb1-3GalNAcb1-4(Neu5Aca2-3)Galb1-4Glcb-Sp9 & 10 & 7 & 68 \\
\hline 248 & Neu5Aca2-3Galb1-3GlcNAcb1-3Galb1-4GIcNAcb-Sp0 & 10 & 4 & 44 \\
\hline 354 & Galb1-4GlcNAcb1-2Mana1-6(Galb1-4GlcNAcb1-2Mana1-3)Manb1-4GlcNAcb1-4(Fuca1-6)GlcNAcb-Sp22 & 10 & 2 & 21 \\
\hline 72 & Fuca1-2Galb1-4(Fuca1-3)GlcNAcb-Sp0 & 9 & 6 & 59 \\
\hline 325 & Galb1-3GIcNAcb1-2Mana1-6(Galb1-3GlcNAcb1-2Mana1-3)Manb1-4GIcNAcb1-4GIcNAcb-Sp19 & 9 & 4 & 39 \\
\hline 567 & GlcNAb1-3Galb1-3GalNAc-Sp14 & 9 & 3 & 35 \\
\hline 472 & Neu5Aca2-3Galb1-4GIcNAcb1-6(Neu5Aca2-3Galb1-4GIcNAcb1-3)GalNAca-Sp14 & 9 & 3 & 36 \\
\hline 77 & Fuca1-2Galb1-4GlcNAcb-Sp8 & 9 & 6 & 63 \\
\hline 549 & $\begin{array}{l}\text { Galb1-4GlcNAcb1-3Galb1-4GIcNAcb1-3Galb1-4GIcNAcb1-2Mana1-6(Galb1-4GIcNAcb1-3Galb1-4GIcNAcb1- } \\
\text { 3Galb1-4GIcNAcb1-2Mana1-3)Manb1-4GlcNAcb1-4GIcNAcb-Sp24 }\end{array}$ & 9 & 10 & 106 \\
\hline 221 & Fuca1-2(6S)Galb1-4GlcNAcb-Sp0 & 9 & 8 & 82 \\
\hline 79 & Fuca1-2Galb-Sp8 & 9 & 8 & 87 \\
\hline 155 & Galb1-4(Fuca1-3)GlcNAcb1-3Galb1-4(Fuca1-3)GlcNAcb1-3Galb1-4(Fuca1-3)GIcNAcb-Sp0 & 9 & 11 & 116 \\
\hline 408 & Galb1-3GIcNAca1-6Galb1-4GIcNAcb-Sp0 & 9 & 6 & 60 \\
\hline 485 & Mana1-6(Mana1-3)Manb1-4GIcNAcb1-4(Fuca1-6)GlcNAcb-Sp19 & 9 & 6 & 61 \\
\hline 448 & Galb1-4(Fuca1-3)GIcNAcb1-6GalNAc-Sp14 & 9 & 10 & 111 \\
\hline 150 & Galb1-3GlcNAcb-Sp0 & 9 & 7 & 72 \\
\hline 342 & GIcNAca1-4Galb1-4GIcNAcb1-3Galb1-4Glcb-Sp0 & 9 & 12 & 127 \\
\hline 441 & Galb1-6Galb-Sp10 & 9 & 12 & 130 \\
\hline 308 & GlcAb1-3GlcNAcb-Sp8 & 9 & 11 & 125 \\
\hline 18 & $\mathrm{GlcN}(\mathrm{Gc}) \mathrm{b}-\mathrm{Sp} 8$ & 9 & 3 & 36 \\
\hline 537 & GalNAca1-3(Fuca1-2)Galb1-3GalNAcb1-3Gala1-4Galb1-4GIc-Sp21 & 9 & 6 & 71 \\
\hline
\end{tabular}




\begin{tabular}{|c|c|c|c|c|}
\hline 391 & Fuca1-2Galb1-3GalNAca1-3(Fuca1-2)Galb1-4GIcNAcb-Sp0 & 9 & 10 & 116 \\
\hline 494 & Galb1-4(Fuca1-3)GlcNAcb1-6(Neu5Aca2-6(Neu5Aca2-3Galb1-3)GlcNAcb1-3)Galb1-4Glc-Sp21 & 9 & 9 & 96 \\
\hline 535 & Neu5Aca2-3Galb1-4(Fuca1-3)GlcNAcb1-2Mana-Sp0 & 9 & 8 & 94 \\
\hline 268 & Neu5Aca2-6Galb1-4(6S)GIcNAcb-Sp8 & 9 & 8 & 92 \\
\hline 356 & (6S)GlcNAcb1-3Galb1-4GIcNAcb-Sp0 & 9 & 3 & 34 \\
\hline 464 & $\begin{array}{l}\text { Neu5Aca2-6Galb1-4GlcNAcb1-2Mana1-6(GIcNAcb1-4)(Neu5Aca2-6Galb1-4GlcNAcb1-2Mana1-3)Manb1- } \\
\text { 4GIcNAcb1-4GlcNAcb-Sp21 }\end{array}$ & 9 & 6 & 64 \\
\hline 206 & KDNa2-3Galb1-3GIcNAcb-Sp0 & 9 & 4 & 40 \\
\hline 59 & Fuca1-2Galb1-3GalNAcb1-3Gala-Sp9 & 9 & 4 & 43 \\
\hline 451 & Gala1-3(Fuca1-2)Galb1-4GIcNAcb1-6(Gala1-3(Fuca1-2)Galb1-4GIcNAcb1-3)GalNAc-Sp14 & 9 & 6 & 63 \\
\hline 611 & Galb1-3GalNAcb1-4(Neu5Aca2-8Neu5Aca2-8Neu5Aca2-3)Galb1-4Glcb-Sp21 & 9 & 6 & 66 \\
\hline 311 & GlcNAcb1-4GIcNAcb-Sp10 & 9 & 4 & 48 \\
\hline 227 & GalNAcb1-4(Neu5Aca2-8Neu5Aca2-8Neu5Aca2-3)Galb1-4Glcb-Sp0 & 9 & 13 & 144 \\
\hline 41 & (6P)Mana-Sp8 & 9 & 6 & 66 \\
\hline 433 & GIcNAcb1-2Mana1-6(GlcNAcb1-4)(GIcNAcb1-4(GIcNAcb1-2)Mana1-3)Manb1-4GIcNAcb1-4GIcNAc-Sp21 & 9 & 10 & 117 \\
\hline 323 & Neu5Aca2-8Neu5Aca2-8Neu5Acb-Sp8 & 9 & 8 & 89 \\
\hline 348 & Neu5Aca2-6Galb1-4GIcNAcb1-2Mana1-6Manb1-4GIcNAcb1-4GIcNAc-Sp12 & 9 & 5 & 57 \\
\hline 40 & (4S)Galb1-4GlcNAcb-Sp8 & 9 & 7 & 84 \\
\hline 351 & Galb1-4GIcNAcb1-2Mana1-6Manb1-4GIcNAcb1-4GIcNAc-Sp12 & 9 & 3 & 40 \\
\hline 124 & Gala1-4GIcNAcb-Sp8 & 9 & 7 & 83 \\
\hline 371 & $\begin{array}{l}\text { GalNAca1-3(Fuca1-2)Galb1-4GIcNAcb1-2Mana1-6(GalNAca1-3(Fuca1-2)Galb1-4GIcNAcb1-2Mana1-3)Manb1- } \\
\text { 4GlcNAcb1-4GlcNAcb-Sp20 }\end{array}$ & 9 & 7 & 79 \\
\hline 254 & Neu5Aca2-3Galb1-4(Fuca1-3)GlcNAcb1-3Galb1-4(Fuca1-3)GlcNAcb1-3Galb1-4(Fuca1-3)GIcNAcb-Sp0 & 9 & 5 & 54 \\
\hline 9 & Neu5Aca-Sp8 & 9 & 2 & 21 \\
\hline 521 & Neu5Aca2-6Galb1-4GIcNAcb1-2Man-Sp0 & 9 & 6 & 69 \\
\hline 158 & Galb1-4GalNAca1-3(Fuca1-2)Galb1-4GlcNAcb-Sp8 & 9 & 13 & 156 \\
\hline 523 & Gala1-3(Fuca1-2)Galb1-4GIcNAcb1-2Mana-Sp0 & 9 & 5 & 56 \\
\hline 171 & Galb1-4GlcNAcb-Sp23 & 9 & 7 & 81 \\
\hline 479 & Neu5Aca2-3Galb1-4GIcNAcb1-2Mana-Sp0 & 8 & 6 & 74 \\
\hline 233 & GalNAcb1-4(Neu5Aca2-3)Galb1-4GlcNAcb-Sp8 & 8 & 4 & 43 \\
\hline 507 & GalNAca1-3(Fuca1-2)Galb1-3GIcNAcb1-6GalNAca-Sp14 & 8 & 10 & 121 \\
\hline 336 & GalNAca1-3(Fuca1-2)Galb1-4GIcNAcb1-3Galb1-4GIcNAcb-Sp0 & 8 & 2 & 28 \\
\hline 525 & Galb1-3GIcNAcb1-2Mana-Sp0 & 8 & 11 & 132 \\
\hline 394 & GalNAca1-3(Fuca1-2)Galb1-3GalNAca1-3(Fuca1-2)Galb1-4GIcNAcb-Sp0 & 8 & 7 & 81 \\
\hline 251 & Neu5Aca2-3Galb1-3GlcNAcb-Sp8 & 8 & 3 & 34 \\
\hline
\end{tabular}




\begin{tabular}{|c|c|c|c|c|}
\hline 181 & GlcNAcb1-3GalNAca-Sp14 & 8 & 8 & 91 \\
\hline 263 & Fuca1-2Galb1-4(6S)Glcb-Sp0 & 8 & 7 & 82 \\
\hline 417 & Fuca1-2Galb1-4(Fuca1-3)GIcNAcb1-3GalNAca-Sp14 & 8 & 7 & 89 \\
\hline 341 & GlcNAca1-4Galb1-3GlcNAcb-Sp0 & 8 & 10 & 120 \\
\hline 258 & Neu5Aca2-3Galb1-4(Fuca1-3)GlcNAcb1-3Galb1-4GlcNAcb-Sp8 & 8 & 4 & 52 \\
\hline 1 & Gala-Sp8 & 8 & 11 & 136 \\
\hline 501 & Fuca1-2Galb1-3(6S)GlcNAcb-Sp0 & 8 & 3 & 38 \\
\hline 20 & Galb1-4GIcNAcb1-6(Galb1-4GlcNAcb1-3)GalNAc-Sp14 & 8 & 7 & 86 \\
\hline 73 & Fuca1-2Galb1-4(Fuca1-3)GIcNAcb-Sp8 & 8 & 2 & 21 \\
\hline 174 & GlcNAca1-3Galb1-4GlcNAcb-Sp8 & 8 & 11 & 129 \\
\hline 385 & Galb1-4GIcNAcb1-6(Fuca1-4(Fuca1-2Galb1-3)GlcNAcb1-3)Galb1-4GIc-Sp21 & 8 & 2 & 30 \\
\hline 510 & Galb1-3GIcNAca1-3Galb1-4GIcNAcb-Sp8 & 8 & 4 & 53 \\
\hline 76 & Fuca1-2Galb1-4GlcNAcb-Sp0 & 8 & 4 & 51 \\
\hline 186 & GlcNAcb1-3Galb1-4Glcb-Sp0 & 8 & 7 & 84 \\
\hline 211 & Mana1-6(Mana1-2Mana1-3)Mana1-6(Mana1-2Mana1-3)Manb1-4GIcNAcb1-4GIcNAcb-Sp12 & 8 & 5 & 64 \\
\hline 456 & $\begin{array}{l}\text { Gala1-3(Fuca1-2)Galb1-3GlcNAcb1-2Mana1-6(Gala1-3(Fuca1-2)Galb1-3GlcNAcb1-2Mana1-3)Manb1- } \\
\text { 4GlcNAcb1-4(Fuca1-6)GlcNAcb-Sp22 }\end{array}$ & 8 & 3 & 36 \\
\hline 276 & Neu5Aca2-8Neu5Aca-Sp8 & 8 & 8 & 98 \\
\hline 322 & Neu5Aca2-8Neu5Acb-Sp17 & 8 & 5 & 60 \\
\hline 330 & Neu5,9Ac2a2-3Galb1-3GIcNAcb-Sp0 & 8 & 5 & 67 \\
\hline 423 & Fuca1-2Galb1-3GIcNAcb1-3GalNAc-Sp14 & 8 & 5 & 70 \\
\hline 55 & $\begin{array}{l}\text { Neu5Aca2-6Galb1-4GIcNAcb1-2Mana1-6(Neu5Aca2-6Galb1-4GIcNAcb1-2Mana1-3)Manb1-4GIcNAcb1- } \\
\text { 4GIcNAcb-Sp12 }\end{array}$ & 8 & 7 & 87 \\
\hline 162 & Galb1-4GIcNAcb1-3Galb1-4(Fuca1-3)GIcNAcb1-3Galb1-4(Fuca1-3)GIcNAcb-Sp0 & 8 & 3 & 36 \\
\hline 591 & Galb1-4GIcNAcb1-3Galb1-4GIcNAcb1-6(Galb1-3)GalNAca-Sp14 & 8 & 7 & 89 \\
\hline 411 & Galb1-4GIcNAcb1-6(Neu5Aca2-6Galb1-3GIcNAcb1-3)Galb1-4Glc-Sp21 & 7 & 5 & 70 \\
\hline 465 & $\begin{array}{l}\text { Neu5Aca2-6Galb1-4GIcNAcb1-4Mana1-6(GlcNAcb1-4)(Neu5Aca2-6Galb1-4GlcNAcb1-4(Neu5Aca2-6Galb1- } \\
\text { 4GIcNAcb1-2)Mana1-3)Manb1-4GIcNAcb1-4GlcNAcb-Sp21 }\end{array}$ & 7 & 5 & 64 \\
\hline 96 & GalNAcb1-3(Fuca1-2)Galb-Sp8 & 7 & 5 & 63 \\
\hline 85 & (3S)Galb1-4(Fuca1-3)Glcb-Sp0 & 7 & 3 & 40 \\
\hline 106 & Gala1-3(Fuca1-2)Galb1-4GlcNAc-Sp0 & 7 & 4 & 55 \\
\hline 487 & $\begin{array}{l}\text { Neu5Aca2-3Galb1-3GIcNAcb1-2Mana1-6(GlcNAcb1-4)(Neu5Aca2-3Galb1-3GlcNAcb1-2Mana1-3)Manb1- } \\
\text { 4GlcNAcb1-4GlcNAc-Sp21 }\end{array}$ & 7 & 5 & 68 \\
\hline 397 & $\begin{array}{l}\text { Neu5Aca2-3Galb1-3GIcNAcb1-2Mana1-6(Neu5Aca2-3Galb1-3GIcNAcb1-2Mana1-3)Manb1-4GIcNAcb1- } \\
\text { 4GIcNAc-Sp19 }\end{array}$ & 7 & 8 & 111 \\
\hline 112 & Gala1-3GalNAca-Sp16 & 7 & 3 & 48 \\
\hline 210 & Mana1-2Mana1-3Mana-Sp9 & 7 & 4 & 61 \\
\hline 298 & (6S)Galb1-4(6S)GIcNAcb-Sp0 & 7 & 1 & 14 \\
\hline 135 & GlcNAcb1-6(Galb1-3)GalNAca-Sp14 & 7 & 5 & 69 \\
\hline
\end{tabular}




\begin{tabular}{|c|c|c|c|c|}
\hline 313 & MurNAcb1-4GIcNAcb-Sp10 & 7 & 6 & 91 \\
\hline 239 & Neu5Aca2-3Galb1-3(6S)GIcNAc-Sp8 & 7 & 2 & 26 \\
\hline 183 & GlcNAcb1-3Galb1-4GlcNAcb-Sp0 & 7 & 3 & 49 \\
\hline 280 & Neu5Acb2-6Galb1-4GlcNAcb-Sp8 & 7 & 11 & 160 \\
\hline 200 & Glcb1-6Glcb-Sp8 & 7 & 3 & 41 \\
\hline 84 & GalNAca1-3(Fuca1-2)Galb1-4(Fuca1-3)GIcNAcb-Sp0 & 7 & 10 & 151 \\
\hline 4 & GalNAca-Sp8 & 7 & 10 & 143 \\
\hline 386 & Galb1-4(Fuca1-3)GIcNAcb1-6(Fuca1-4(Fuca1-2Galb1-3)GlcNAcb1-3)Galb1-4GIc-Sp21 & 7 & 5 & 83 \\
\hline 21 & GlcNAcb1-6(GlcNAcb1-4)(GlcNAcb1-3)GlcNAc-Sp8 & 7 & 1 & 16 \\
\hline 203 & GlcAb-Sp8 & 7 & 11 & 176 \\
\hline 310 & GlcNAcb1-3Man-Sp10 & 7 & 1 & 16 \\
\hline 139 & Neu5Aca2-6(Galb1-3)GlcNAcb1-4Galb1-4Glcb-Sp10 & 7 & 5 & 69 \\
\hline 53 & GlcNAcb1-2Mana1-6(GlcNAcb1-2Mana1-3)Manb1-4GIcNAcb1-4GIcNAcb-Sp13 & 6 & 6 & 100 \\
\hline 88 & GalNAca1-3(Fuca1-2)Galb1-4Glcb-Sp0 & 6 & 8 & 127 \\
\hline 275 & Neu5Aca2-6Galb-Sp8 & 6 & 9 & 145 \\
\hline 145 & Galb1-3GalNAcb1-4(Neu5Aca2-3)Galb1-4GIcb-Sp0 & 6 & 4 & 72 \\
\hline 435 & $\begin{array}{l}\text { GlcNAcb1-6(GlcNAcb1-2)Mana1-6(GlcNAcb1-4)(GlcNAcb1-4(GlcNAcb1-2)Mana1-3)Manb1-4GlcNAcb1- } \\
\text { 4GlcNAc-Sp21 }\end{array}$ & 6 & 4 & 60 \\
\hline 163 & Galb1-4GlcNAcb1-3Galb1-4GlcNAcb1-3Galb1-4GlcNAcb-Sp0 & 6 & 3 & 51 \\
\hline 540 & $\begin{array}{l}\text { GlcNAcb1-3Galb1-4GlcNAcb1-2Mana1-6(GlcNAcb1-3Galb1-4GIcNAcb1-2Mana1-3)Manb1-4GlcNAcb1- } \\
\text { 4GIcNAcb-Sp12 }\end{array}$ & 6 & 4 & 70 \\
\hline 527 & Neu5Aca2-3Galb1-3GIcNAcb1-2Mana-Sp0 & 6 & 3 & 47 \\
\hline 401 & Fuca1-2Galb1-4GlcNAcb1-3GalNAca-Sp14 & 6 & 7 & 118 \\
\hline 530 & Neu5Aca2-3Galb1-3GlcNAcb1-4Galb1-4Glcb-Sp0 & 6 & 5 & 86 \\
\hline 346 & Neu5Aca2-6Galb1-4GIcNAcb1-2Mana1-6(Mana1-3)Manb1-4GIcNAcb1-4GIcNAc-Sp12 & 6 & 5 & 78 \\
\hline 39 & $(6 S)(4 S)$ Galb1-4GIcNAcb-Sp0 & 6 & 5 & 86 \\
\hline 191 & GlcNAcb1-4GlcNAcb1-4GlcNAcb1-4GlcNAcb1-4GIcNAcb1-Sp8 & 6 & 7 & 127 \\
\hline 136 & Neu5Aca2-6(Galb1-3)GalNAca-Sp8 & 6 & 7 & 118 \\
\hline 304 & GlcNAcb1-6(Galb1-4GIcNAcb1-3)Galb1-4GIcNAc-Sp0 & 6 & 5 & 85 \\
\hline 202 & GlcAa-Sp8 & 6 & 6 & 113 \\
\hline 430 & Galb1-4GIcNAcb1-6(Fuca1-2Galb1-3GIcNAcb1-3)Galb1-4GIc-Sp21 & 6 & 5 & 97 \\
\hline 177 & GlcNAcb1-6(GIcNAcb1-3)GalNAca-Sp8 & 6 & 11 & 189 \\
\hline 489 & Galb1-3GIcNAcb1-6GalNAca-Sp14 & 6 & 3 & 54 \\
\hline 32 & (3S)Galb1-4(Fuca1-3)GlcNAc-Sp0 & 6 & 18 & 331 \\
\hline 508 & $\begin{array}{l}\text { GlcNAcb1-6(GlcNAcb1-2)Mana1-6(GlcNAcb1-4)(GlcNAcb1-4(GlcNAcb1-2)Mana1-3)Manb1-4GlcNAcb1-4(Fuca1- } \\
\text { 6)GIcNAc-Sp21 }\end{array}$ & 6 & 1 & 19 \\
\hline 81 & Fuca1-4GlcNAcb-Sp8 & 6 & 6 & 104 \\
\hline
\end{tabular}




\begin{tabular}{|c|c|c|c|c|}
\hline 335 & GalNAcb1-3Gala1-4Galb1-4GIcNAcb1-3Galb1-4Glcb-Sp0 & 6 & 7 & 130 \\
\hline 350 & Galb1-4GIcNAcb1-2Mana1-3Manb1-4GlcNAcb1-4GlcNAc-Sp12 & 5 & 6 & 110 \\
\hline 109 & Gala1-3(Fuca1-2)Galb-Sp18 & 5 & 9 & 166 \\
\hline 87 & GalNAca1-3(Fuca1-2)Galb1-4GIcNAcb-Sp8 & 5 & 6 & 106 \\
\hline 142 & Galb1-3GalNAca-Sp16 & 5 & 3 & 57 \\
\hline 414 & Gala1-3(Fuca1-2)Galb1-4GIcNAcb1-3GalNAca-Sp14 & 5 & 4 & 72 \\
\hline 195 & GlcNAcb1-6Galb1-4GlcNAcb-Sp8 & 5 & 12 & 225 \\
\hline 188 & GlcNAcb1-6(GlcNAcb1-4)GalNAca-Sp8 & 5 & 7 & 133 \\
\hline 339 & GlcNAca1-4Galb1-4GlcNAcb1-3Galb1-4GlcNAcb1-3Galb1-4GIcNAcb-Sp0 & 5 & 8 & 165 \\
\hline 176 & GlcNAcb1-2Galb1-3GalNAca-Sp8 & 5 & 6 & 121 \\
\hline 70 & Fuca1-2Galb1-4(Fuca1-3)GlcNAcb1-3Galb1-4(Fuca1-3)GlcNAcb-Sp0 & 5 & 6 & 123 \\
\hline 609 & $\begin{array}{l}\text { Neu5Aca2-6Galb1-4GlcNAcb1-3Galb1-4GlcNAcb1-2Mana1-6(Neu5Aca2-6Galb1-4GlcNAcb1-3Galb1- } \\
\text { 4GlcNAcb1-2Mana1-3)Manb1-4GlcNAcb1-4GlcNAcb-Sp12 }\end{array}$ & 5 & 6 & 113 \\
\hline 60 & Fuca1-2Galb1-3GalNAcb1-3Gala1-4Galb1-4GIcb-Sp9 & 5 & 9 & 188 \\
\hline 2 & Glca-Sp8 & 5 & 3 & 55 \\
\hline 425 & GalNAca1-3(Fuca1-2)Galb1-3GIcNAcb1-3GalNAc-Sp14 & 5 & 7 & 143 \\
\hline 455 & $\begin{array}{l}\text { GalNAca1-3(Fuca1-2)Galb1-4GIcNAcb1-2Mana1-6(GalNAca1-3(Fuca1-2)Galb1-4GlcNAcb1-2Mana1-3)Manb1- } \\
\text { 4GlcNAcb1-4(Fuca1-6)GlcNAcb-Sp22 }\end{array}$ & 5 & 2 & 53 \\
\hline 128 & Galb1-3GlcNAcb1-3Galb1-4(Fuca1-3)GlcNAcb-Sp0 & 5 & 3 & 59 \\
\hline 301 & Galb1-3Galb1-4GlcNAcb-Sp8 & 4 & 10 & 220 \\
\hline 399 & Galb1-4GIcNAcb1-2Mana1-6(GIcNAcb1-2Mana1-3)Manb1-4GIcNAcb1-4GIcNAc-Sp12 & 4 & 5 & 127 \\
\hline 273 & Neu5Aca2-6Galb1-4GIcb-Sp0 & 4 & 3 & 75 \\
\hline 266 & Neu5Aca2-6GalNAca-Sp8 & 4 & 7 & 166 \\
\hline 214 & Mana1-6(Mana1-3)Mana-Sp9 & 4 & 4 & 101 \\
\hline 141 & Galb1-3GalNAca-Sp14 & 4 & 2 & 48 \\
\hline 161 & Galb1-4GIcNAcb1-3GalNAc-Sp14 & 4 & 2 & 51 \\
\hline 269 & Neu5Aca2-6Galb1-4GIcNAcb-Sp0 & 4 & 7 & 181 \\
\hline 353 & GIcNAcb1-2Mana1-6(GIcNAcb1-2Mana1-3)Manb1-4GlcNAcb1-4(Fuca1-6)GIcNAcb-Sp22 & 4 & 3 & 78 \\
\hline 294 & Galb1-4GIcNAcb1-3Galb1-3GIcNAcb-Sp0 & 4 & 6 & 143 \\
\hline 300 & Neu5Aca2-3Galb1-4(Fuca1-3)GlcNAcb1-6(Galb1-3)GalNAca-Sp14 & 4 & 3 & 63 \\
\hline 140 & Galb1-3GalNAca-Sp8 & 4 & 3 & 72 \\
\hline 185 & GlcNAcb1-3Galb1-4GlcNAcb1-3Galb1-4GlcNAcb-Sp0 & 4 & 5 & 125 \\
\hline
\end{tabular}




\begin{tabular}{|c|c|c|c|c|}
\hline 115 & Gala1-3Galb1-3GlcNAcb-Sp0 & 4 & 10 & 268 \\
\hline 367 & Neu5Aca2-6GlcNAcb1-4GlcNAc-Sp21 & 4 & 2 & 67 \\
\hline 101 & Gala1-2Galb-Sp8 & 4 & 3 & 95 \\
\hline 321 & GlcNAcb1-2Mana1-6(Neu5Aca2-6Galb1-4GIcNAcb1-2Mana1-3)Manb1-4GlcNAcb1-4GlcNAcb-Sp12 & 4 & 3 & 73 \\
\hline 256 & Neu5Aca2-3Galb1-4(Fuca1-3)GlcNAcb-Sp8 & 3 & 5 & 158 \\
\hline 519 & (6P)Galb1-4GlcNAcb-SP0 & 3 & 4 & 108 \\
\hline 281 & Neu5Gca2-3Galb1-3(Fuca1-4)GlcNAcb-Sp0 & 3 & 7 & 214 \\
\hline 62 & Fuca1-2Galb1-3GalNAca-Sp8 & 3 & 4 & 136 \\
\hline 197 & Glca1-4Glca-Sp8 & 3 & 6 & 211 \\
\hline 288 & Neu5Gca-Sp8 & 3 & 7 & 238 \\
\hline 242 & Neu5Aca2-3Galb1-4(Neu5Aca2-3Galb1-3)GlcNAcb-Sp8 & 3 & 6 & 222 \\
\hline 178 & GlcNAcb1-6(GlcNAcb1-3)GalNAca-Sp14 & 3 & 2 & 79 \\
\hline 381 & Galb1-3GalNAca1-3(Fuca1-2)Galb1-4Glc-Sp0 & 3 & 3 & 99 \\
\hline 192 & GIcNAcb1-4GlcNAcb1-4GlcNAcb-Sp8 & 3 & 3 & 98 \\
\hline 170 & Galb1-4GlcNAcb-Sp8 & 3 & 4 & 141 \\
\hline 223 & Fuca1-2(6S)Galb1-4(6S)Glcb-Sp0 & 2 & 1 & 33 \\
\hline 379 & Neu5Aca2-3Galb1-4(Fuca1-3)GIcNAcb1-3GalNAca-Sp14 & 2 & 3 & 124 \\
\hline 345 & GIcNAca1-4Galb1-3GalNAc-Sp14 & 2 & 10 & 444 \\
\hline 218 & Manb1-4GIcNAcb-Sp0 & 2 & 4 & 185 \\
\hline 410 & Gala1-3(Fuca1-2)Galb1-4(Fuca1-3)Glcb-Sp21 & 2 & 6 & 279 \\
\hline 557 & Neu5Gca2-8Neu5Gca2-6Galb1-4GıcNAc-Sp0 & 2 & 2 & 129 \\
\hline 267 & Neu5Aca2-6GalNAcb1-4GlcNAcb-Sp0 & 2 & 7 & 369 \\
\hline 518 & Galb1-4(6P)GlcNAcb-Sp0 & 2 & 6 & 341 \\
\hline 482 & Neu5Aca2-6Galb1-4 GlcNAcb1-6(Neu5Aca2-6Galb1-4GIcNAcb1-3)GalNAca-Sp14 & 1 & 2 & 133 \\
\hline 443 & GalNAcb1-6GalNAcb-Sp8 & 1 & 6 & 414 \\
\hline 120 & Gala1-4(Fuca1-2)Galb1-4GIcNAcb-Sp8 & 1 & 2 & 157 \\
\hline 302 & Neu5Aca2-6Galb1-4GIcNAcb1-2Mana1-6(Galb1-4GIcNAcb1-2Mana1-3)Manb1-4GIcNAcb1-4GlcNAcb-Sp12 & 1 & 2 & 151 \\
\hline 524 & GalNAca1-3(Fuca1-2)Galb1-4 GlcNAcb1-2Mana-Sp0 & 1 & 4 & 499 \\
\hline 403 & GalNAca1-3GalNAcb1-3Gala1-4Galb1-4GIcNAcb-Sp0 & 0 & 7 & 2695 \\
\hline
\end{tabular}




\begin{tabular}{|c|l|c|c|c|}
334 & Gala1-4Galb1-4GIcNAcb1-3Galb1-4GIcb-Sp0 & -1 & 1 & -216 \\
\hline 204 & GIcAb1-3Galb-Sp8 & -1 & 5 & -712 \\
\hline
\end{tabular}

Supporting data 3. AAL-20ug_14924_V5.0_DATA

\begin{tabular}{|c|c|c|c|c|}
\hline $\begin{array}{l}\text { Chart } \\
\text { Number }\end{array}$ & AAL (20ug/ml) CFG\#2473 Slide\#:14924 12/19/2011 Alexa488 CMZ & Average RFU & StDev & $\% \mathrm{CV}$ \\
\hline 570 & (3S)GlcAb1-3Galb1-4GIcNAcb1-3Galb1-4GIc-Sp0 & 2739 & 286 & 10 \\
\hline 571 & (3S)GlcAb1-3Galb1-4GlcNAcb1-2Mana-Sp0 & 1293 & 38 & 3 \\
\hline 29 & (3S)Galb1-3GalNAca-Sp8 & 615 & 42 & 7 \\
\hline 554 & Neu5Aca2-8Neu5Gca2-3Galb1-4GıcNAc-Sp0 & 259 & 35 & 13 \\
\hline 561 & Gala1-3Galb1-4GIcNAcb1-2Mana1-6(Gala1-3Galb1-4GIcNAcb1-2Mana1-3)Manb1-4GIcNAcb1-4GIcNAc-Sp24 & 159 & 21 & 13 \\
\hline 364 & Gala1-3Galb1-4GIcNAcb1-2Mana1-6(Gala1-3Galb1-4GIcNAcb1-2Mana1-3)Manb1-4GIcNAcb1-4GIcNAcb-Sp20 & 107 & 26 & 24 \\
\hline 110 & Gala1-4(Gala1-3)Galb1-4GIcNAcb-Sp8 & 55 & 16 & 29 \\
\hline 45 & (6S)Galb1-4(6S)Glcb-Sp8 & 39 & 26 & 68 \\
\hline 475 & $\begin{array}{l}\text { Neu5Aca2-3Galb1-3GlcNAcb1-6(Neu5Aca2-3Galb1-4GlcNAcb1-2)Mana1-6(Neu5Aca2-3Galb1-3GlcNAcb1- } \\
\text { 2Mana1-3)Manb1-4GlcNAcb1-4GlcNAcb-Sp19 }\end{array}$ & 38 & 23 & 61 \\
\hline 548 & $\begin{array}{l}\text { Galb1-4GIcNAcb1-3Galb1-4GIcNAcb1-3Galb1-4GIcNAcb1-2Mana1-6(Galb1-4GIcNAcb1-3Galb1-4GIcNAcb1- } \\
\text { 3Galb1-4GIcNAcb1-2Mana1-3)Manb1-4GIcNAcb1-4GIcNAcb-Sp12 }\end{array}$ & 37 & 20 & 53 \\
\hline 578 & $\begin{array}{l}\text { Galb1-4GIcNAcb1-3Galb1-4GIcNAcb1-3Galb1-4GIcNAcb1-2Mana1-6(Galb1-4GIcNAcb1-3Galb1-4GIcNAcb1- } \\
\text { 3Galb1-4GIcNAcb1-2Mana1-3)Manb1-4GIcNAcb1-4(Fuca1-6)GIcNAcb-Sp24 }\end{array}$ & 36 & 6 & 15 \\
\hline 12 & Galb-Sp8 & 36 & 7 & 20 \\
\hline 338 & Neu5Aca2-3Galb1-4(Fuca1-3)GlcNAcb1-6(Neu5Aca2-3Galb1-3)GalNAc-Sp14 & 35 & 15 & 44 \\
\hline 336 & GalNAca1-3(Fuca1-2)Galb1-4GIcNAcb1-3Galb1-4GIcNAcb-Sp0 & 35 & 27 & 78 \\
\hline 361 & Fuca1-2Galb1-3GIcNAcb1-2Mana1-6(Fuca1-2Galb1-3GIcNAcb1-2Mana1-3)Manb1-4GIcNAcb1-4GIcNAcb-Sp20 & 32 & 9 & 27 \\
\hline 229 & GalNAcb1-4(Neu5Aca2-8Neu5Aca2-3)Galb1-4Glcb-Sp0 & 32 & 14 & 43 \\
\hline 586 & $\begin{array}{l}\text { GlcNAcb1-3Galb1-4GlcNAcb1-3Galb1-4GlcNAcb1-3Galb1-4GlcNAcb1-6(GlcNAcb1-3Galb1-4GlcNAcb1-3Galb1- } \\
\text { 4GlcNAcb1-3Galb1-4GlcNAb1-2)Mana1-6(GIcNAcb1-3Galb1-4GlcNAcb1-3Galb1-4GlcNAcb1-3Galb1- } \\
\text { 4GlcNAcb1-2Mana1-3)Manb1-4GlcNAcb1-4(Fuca1-6)GlcNAcb-Sp24 }\end{array}$ & 32 & 20 & 62 \\
\hline 426 & Gala1-3Galb1-3GIcNAcb1-3GalNAc-Sp14 & 31 & 43 & 141 \\
\hline 545 & $\begin{array}{l}\text { Fuca1-2Galb1-4GIcNAcb1-3Galb1-4GIcNAcb1-2Mana1-6(Fuca1-2Galb1-4GlcNAcb1-3Galb1-4GIcNAcb1- } \\
\text { 2Mana1-3)Manb1-4GIcNAcb1-4GIcNAcb-Sp24 }\end{array}$ & 29 & 21 & 70 \\
\hline 408 & Galb1-3GlcNAca1-6Galb1-4GIcNAcb-Sp0 & 29 & 18 & 62 \\
\hline 115 & Gala1-3Galb1-3GlcNAcb-Sp0 & 29 & 11 & 37 \\
\hline 585 & $\begin{array}{l}\text { Galb1-4GIcNAcb1-3Galb1-4GIcNAcb1-3Galb1-4GlcNAcb1-6(Galb1-4GIcNAcb1-3Galb1-4GIcNAcb1-3Galb1- } \\
\text { 4GlcNAb1-2)Mana1-6(Galb1-4GIcNAcb1-3Galb1-4GIcNAcb1-3Galb1-4GlcNAcb1-2Mana1-3)Manb1-4GIcNAcb1- } \\
\text { 4(Fuca1-6)GIcNAcb-Sp24 }\end{array}$ & 29 & 12 & 42 \\
\hline 337 & GalNAca1-3(Fuca1-2)Galb1-4GIcNAcb1-3Galb1-4GIcNAcb1-3Galb1-4GIcNAcb-Sp0 & 28 & 15 & 54 \\
\hline 217 & Mana1-6(Mana1-3)Mana1-6(Mana1-3)Manb1-4GIcNAcb1-4GIcNAcb-Sp12 & 28 & 23 & 82 \\
\hline 484 & $\begin{array}{l}\text { Neu5Aca2-3Galb1-4GIcNAcb1-2Mana1-6(Neu5Aca2-3Galb1-4GIcNAcb1-2Mana1-3)Manb1-4GIcNAcb1-4(Fuca1- } \\
\text { 6)GIcNAcb-Sp24 }\end{array}$ & 28 & 9 & 32 \\
\hline 285 & Neu5Gca2-3Galb1-4Glcb-Sp0 & 28 & 13 & 47 \\
\hline
\end{tabular}




\begin{tabular}{|c|c|c|c|c|}
\hline 421 & $\begin{array}{l}\text { Fuca1-2Galb1-4GIcNAcb1-2Mana1-6(Fuca1-2Galb1-4GIcNAcb1-2Mana1-3)Manb1-4GIcNAcb1-4(Fuca1- } \\
\text { 6)GIcNAcb-Sp22 }\end{array}$ & 28 & 10 & 34 \\
\hline 340 & GlcNAca1-4Galb1-4GlcNAcb-Sp0 & 28 & 13 & 48 \\
\hline 579 & $\begin{array}{l}\text { GlcNAcb1-3Galb1-4GlcNAcb1-3Galb1-4GlcNAcb1-3Galb1-4GlcNAcb1-2Mana1-6(GlcNAcb1-3Galb1-4GIcNAcb1- } \\
\text { 3Galb1-4GlcNAcb1-3Galb1-4GIcNAcb1-2Mana1-3)Manb1-4GlcNAcb1-4(Fuca1-6)GlcNAcb-Sp24 }\end{array}$ & 28 & 6 & 23 \\
\hline 521 & Neu5Aca2-6Galb1-4GIcNAcb1-2Man-Sp0 & 28 & 9 & 31 \\
\hline 450 & Fuca1-2Galb1-4GIcNAcb1-6(Fuca1-2Galb1-4GIcNAcb1-3)GalNAc-Sp14 & 27 & 19 & 68 \\
\hline 57 & $\begin{array}{l}\text { Neu5Aca2-6Galb1-4GIcNAcb1-2Mana1-6(Neu5Aca2-6Galb1-4GIcNAcb1-2Man-a1-3)Manb1-4GIcNAcb1- } \\
\text { 4GIcNAcb-Sp21 }\end{array}$ & 27 & 9 & 32 \\
\hline 565 & $\begin{array}{l}\text { GlcNAcb1-3Galb1-4GIcNAcb1-3Galb1-4GIcNAcb1-3Galb1-4GlcNAcb1-3Galb1-4GIcNAcb1-2Mana1-6(GlcNAcb1- } \\
\text { 3Galb1-4GIcNAcb1-3Galb1-4GIcNAcb1-3Galb1-4GIcNAcb1-3Galb1-4GIcNAcb1-2Mana1-3)Manb1-4GIcNAcb1- } \\
\text { 4GIcNAcb-Sp25 }\end{array}$ & 25 & 8 & 33 \\
\hline 326 & $\begin{array}{l}\text { Neu5Aca2-3Galb1-4GIcNAcb1-2Mana1-6(Neu5Aca2-3Galb1-4GIcNAcb1-2Mana1-3)Manb1-4GIcNAcb1- } \\
\text { 4GIcNAcb-Sp12 }\end{array}$ & 25 & 9 & 37 \\
\hline 454 & GalNAcb1-4Galb1-4GIcb-Sp0 & 25 & 20 & 81 \\
\hline 99 & GalNAcb1-4GlcNAcb-Sp0 & 25 & 15 & 59 \\
\hline 559 & $\begin{array}{l}\text { GlcNAcb1-3Galb1-4GlcNAcb1-6(GlcNAcb1-3Galb1-4GlcNAcb1-2)Mana1-6(GlcNAcb1-3Galb1-4GlcNAcb1- } \\
\text { 2Man a1-3)Manb1-4GlcNAcb1-4GlcNAc-Sp24 }\end{array}$ & 25 & 10 & 42 \\
\hline 7 & Fuca-Sp9 & 24 & 15 & 61 \\
\hline 577 & $\begin{array}{l}\text { GIcNAcb1-3Galb1-4GIcNAcb1-3Galb1-4GIcNAcb1-2Mana1-6(GIcNAcb1-3Galb1-4GIcNAcb1-3Galb1-4GIcNAcb1- } \\
\text { 2Mana1-3)Manb1-4GIcNAcb1-4(Fuca1-6)GIcNAcb-Sp24 }\end{array}$ & 24 & 6 & 25 \\
\hline 30 & (3S)Galb1-3GlcNAcb-Sp0 & 24 & 25 & 107 \\
\hline 343 & GIcNAca1-4Galb1-4GIcNAcb1-3Galb1-4(Fuca1-3)GlcNAcb1-3Galb1-4(Fuca1-3)GIcNAcb-Sp0 & 24 & 11 & 47 \\
\hline 98 & GalNAcb1-4(Fuca1-3)GlcNAcb-Sp0 & 24 & 13 & 56 \\
\hline 471 & Glca1-4Glca1-4Glca1-4Glcb-Sp10 & 23 & 12 & 51 \\
\hline 20 & Galb1-4GIcNAcb1-6(Galb1-4GlcNAcb1-3)GalNAc-Sp14 & 23 & 9 & 39 \\
\hline 460 & $\begin{array}{l}\text { Neu5Aca2-3Galb1-4GIcNAcb1-2Mana1-6(GlcNAcb1-4)(Neu5Aca2-3Galb1-4GIcNAcb1-2Mana1-3)Manb1- } \\
\text { 4GIcNAcb1-4GlcNAcb-Sp21 }\end{array}$ & 23 & 6 & 28 \\
\hline 537 & GalNAca1-3(Fuca1-2)Galb1-3GalNAcb1-3Gala1-4Galb1-4GIc-Sp21 & 23 & 23 & 100 \\
\hline 175 & GlcNAca1-6Galb1-4GlcNAcb-Sp8 & 23 & 13 & 57 \\
\hline 477 & Galb1-3GIcNAcb1-2Mana1-6(GIcNAcb1-4)(Galb1-3GIcNAcb1-2Mana1-3)Manb1-4GIcNAcb1-4GIcNAcb-Sp21 & 23 & 7 & 30 \\
\hline 366 & $\begin{array}{l}\text { Fuca1-4(Galb1-3)GIcNAcb1-2Mana1-6(Fuca1-4(Galb1-3)GIcNAcb1-2Mana1-3)Manb1-4GIcNAcb1-4(Fuca1- } \\
\text { 6)GIcNAcb-Sp22 }\end{array}$ & 22 & 10 & 45 \\
\hline 474 & $\begin{array}{l}\text { Fuca1-2Galb1-3(Fuca1-4)GIcNAcb1-2Mana1-6(Fuca1-2Galb1-3(Fuca1-4)GlcNAcb1-2Mana1-3)Manb1- } \\
\text { 4GIcNAcb1-4(Fuca1-6)GIcNAcb1-4(Fuca1-6)GIcNAcb-Sp19 }\end{array}$ & 22 & 12 & 52 \\
\hline 64 & Fuca1-2Galb1-3GalNAcb1-4(Neu5Aca2-3)Galb1-4Glcb-Sp0 & 22 & 14 & 64 \\
\hline 476 & GlcNAcb1-6(GlcNAcb1-2)Mana1-6(GlcNAcb1-2Mana1-3)Manb1-4GIcNAcb1-4(Fuca1-6)GIcNAcb-Sp24 & 22 & 8 & 34 \\
\hline 379 & Neu5Aca2-3Galb1-4(Fuca1-3)GIcNAcb1-3GalNAca-Sp14 & 22 & 13 & 61 \\
\hline 488 & Neu5Aca2-6Galb1-4GIcNAcb1-6(Fuca1-2Galb1-4(Fuca1-3)GlcNAcb1-3)Galb1-4Glc-Sp21 & 22 & 12 & 57 \\
\hline 542 & $\begin{array}{l}\text { Galb1-4GIcNAcb1-3Galb1-4GlcNAcb1-2Mana1-6(Galb1-4GIcNAcb1-3Galb1-4GIcNAcb1-2Mana1-3)Manb1- } \\
\text { 4GlcNAcb1-4GIcNAcb-Sp12 }\end{array}$ & 22 & 12 & 55 \\
\hline 447 & $\begin{array}{l}\text { Fuca1-2Galb1-4(Fuca1-3)GIcNAcb1-2Mana1-6(Fuca1-2Galb1-4(Fuca1-3)GlcNAcb1-4(Fuca1-2Galb1-4(Fuca1- } \\
\text { 3)GIcNAcb1-2)Mana1-3)Manb1-4GIcNAcb1-4GIcNAcb-Sp12 }\end{array}$ & 21 & 8 & 37 \\
\hline
\end{tabular}




\begin{tabular}{|c|c|c|c|c|}
\hline 527 & Neu5Aca2-3Galb1-3GIcNAcb1-2Mana-Sp0 & 21 & 16 & 74 \\
\hline 264 & Neu5Aca2-3Galb1-4GIcb-Sp0 & 21 & 21 & 98 \\
\hline 569 & $\begin{array}{l}\text { Galb1-4GlcNAcb1-3Galb1-4GlcNAcb1-3Galb1-4GlcNAcb1-3Galb1-4GlcNAcb1-3Galb1-4GlcNAcb1-3Galb1- } \\
\text { 4GlcNAcb1-2Mana1-6(Galb1-4GlcNAcb1-3Galb1-4GlcNAcb1-3Galb1-4GlcNAcb1-3Galb1-4GlcNAcb1-3Galb1- } \\
\text { 4GlcNAcb1-3Galb1-4GlcNAcb1-2Mana1-3)Manb1-4GlcNAcb1-4GlcNAcb-Sp25 }\end{array}$ & 21 & 9 & 43 \\
\hline 196 & Glca1-4Glcb-Sp8 & 21 & 2 & 10 \\
\hline 243 & Neu5Aca2-3Galb1-3(6S)GalNAca-Sp8 & 21 & 9 & 40 \\
\hline 315 & Mana1-6(Mana1-3)Mana1-6(Mana1-3)Manb-Sp10 & 21 & 14 & 64 \\
\hline 580 & $\begin{array}{l}\text { Galb1-4GIcNAcb1-3Galb1-4GIcNAcb1-3Galb1-4GIcNAcb1-3Galb1-4GIcNAcb1-2Mana1-6(Galb1-4GIcNAcb1- } \\
\text { 3Galb1-4GIcNAcb1-3Galb1-4GIcNAcb1-3Galb1-4GIcNAcb1-2Mana1-3)Manb1-4GIcNAcb1-4(Fuca1-6)GlcNAcb- } \\
\text { Sp24 }\end{array}$ & 21 & 16 & 77 \\
\hline 149 & Galb1-3GIcNAcb1-3Galb1-4GIcb-Sp10 & 21 & 7 & 34 \\
\hline 589 & $\begin{array}{l}\text { Galb1-4GlcNAcb1-3Galb1-4GlcNAcb1-3Galb1-4GlcNAcb1-3Galb1-4GlcNAcb1-3Galb1-4GlcNAcb1-6(Galb1- } \\
\text { 4GlcNAcb1-3Galb1-4GlcNAcb1-3Galb1-4GlcNAcb1-3Galb1-4GlcNAcb1-3Galb1-4GlcNAb1-2)Mana1-6(Galb1- } \\
\text { 4GlcNAcb1-3Galb1-4GlcNAcb1-3Galb1-4GlcNAcb1-3Galb1-4GlcNAcb1-3Galb1-4GlcNAcb1-2Mana1-3)Manb1- } \\
\text { 4GlcNAcb1-4(Fuca1-6)GlcNAcb-Sp24 }\end{array}$ & 21 & 10 & 46 \\
\hline 427 & $\begin{array}{l}\text { Fuca1-2Galb1-3GIcNAcb1-2Mana1-6(Fuca1-2Galb1-3GIcNAcb1-2Mana1-3)Manb1-4GIcNAcb1-4(Fuca1- } \\
\text { 6)GIcNAcb-Sp22 }\end{array}$ & 21 & 4 & 18 \\
\hline 156 & Galb1-4(6S)Glcb-Sp0 & 21 & 17 & 83 \\
\hline 409 & GalNAcb1-3Gala1-6Galb1-4Glcb-Sp8 & 21 & 6 & 27 \\
\hline 183 & GlcNAcb1-3Galb1-4GlcNAcb-Sp0 & 20 & 12 & 57 \\
\hline 348 & Neu5Aca2-6Galb1-4GIcNAcb1-2Mana1-6Manb1-4GIcNAcb1-4GIcNAc-Sp12 & 20 & 13 & 66 \\
\hline 236 & Neu5Aca2-6(Neu5Aca2-3)GalNAca-Sp8 & 20 & 8 & 42 \\
\hline 200 & Glcb1-6Glcb-Sp8 & 20 & 19 & 93 \\
\hline 79 & Fuca1-2Galb-Sp8 & 20 & 10 & 50 \\
\hline 468 & Gala1-3(Fuca1-2)Galb1-3GalNAca-Sp8 & 20 & 11 & 54 \\
\hline 413 & Neu5Aca2-3Galb1-3GalNAcb1-4(Neu5Aca2-8Neu5Aca2-3)Galb1-4Glcb-Sp0 & 20 & 19 & 95 \\
\hline 587 & $\begin{array}{l}\text { Galb1-4GlcNAcb1-3Galb1-4GIcNAcb1-3Galb1-4GIcNAcb1-3Galb1-4GlcNAcb1-6(Galb1-4GlcNAcb1-3Galb1- } \\
\text { 4GIcNAcb1-3Galb1-4GlcNAcb1-3Galb1-4GlcNAb1-2)Mana1-6(Galb1-4GIcNAcb1-3Galb1-4GlcNAcb1-3Galb1- } \\
\text { 4GIcNAcb1-3Galb1-4GlcNAcb1-2Mana1-3)Manb1-4GlcNAcb1-4(Fuca1-6)GlcNAcb-Sp24 }\end{array}$ & 20 & 5 & 27 \\
\hline 276 & Neu5Aca2-8Neu5Aca-Sp8 & 20 & 19 & 94 \\
\hline 37 & (3S)Galb1-4GIcNAcb-Sp8 & 20 & 7 & 34 \\
\hline 245 & Neu5Aca2-6(Neu5Aca2-3Galb1-3)GalNAca-Sp14 & 20 & 20 & 100 \\
\hline 457 & Neu5Aca2-6Galb1-4GIcNAcb1-6(Fuca1-2Galb1-3GlcNAcb1-3)Galb1-4GIc-Sp21 & 20 & 11 & 57 \\
\hline 199 & Glcb1-4Glcb-Sp8 & 20 & 13 & 66 \\
\hline 1 & Gala-Sp8 & 20 & 11 & 54 \\
\hline 541 & $\begin{array}{l}\text { GlcNAcb1-3Galb1-4GIcNAcb1-2Mana1-6(GlcNAcb1-3Galb1-4GIcNAcb1-2Mana1-3)Manb1-4GIcNAcb1- } \\
\text { 4GIcNAcb-Sp25 }\end{array}$ & 20 & 14 & 72 \\
\hline 372 & $\begin{array}{l}\text { Gala1-3(Fuca1-2)Galb1-4GIcNAcb1-2Mana1-6(Gala1-3(Fuca1-2)Galb1-4GIcNAcb1-2Mana1-3)Manb1- } \\
\text { 4GlcNAcb1-4GIcNAcb-Sp20 }\end{array}$ & 20 & 16 & 79 \\
\hline 500 & Fuca1-2Galb1-4GIcNAcb1-2Mana-Sp0 & 20 & 17 & 87 \\
\hline 467 & $\begin{array}{l}\text { Neu5Aca2-6Galb1-4GlcNAcb1-6(Neu5Aca2-6Galb1-4GlcNAcb1-2)Mana1-6(GlcNAcb1-4)(Neu5Aca2-6Galb1- } \\
\text { 4GIcNAcb1-4(Neu5Aca2-6Galb1-4GIcNAcb1-2)Mana1-3)Manb1-4GIcNAcb1-4GIcNAcb-Sp21 }\end{array}$ & 20 & 5 & 23 \\
\hline
\end{tabular}




\begin{tabular}{|c|c|c|c|c|}
\hline 573 & $\begin{array}{l}\text { Galb1-3GlcNAcb1-3Galb1-4GIcNAcb1-6(Galb1-3GIcNAcb1-3Galb1-4GlcNAb1-2)Mana1-6(Galb1-3GlcNAcb1- } \\
\text { 3Galb1-4GlcNAcb1-2Mana1-3)Manb1-4GIcNAcb1-4(Fuca1-6)GlcNAcb-Sp24 }\end{array}$ & 19 & 7 & 38 \\
\hline 172 & Galb1-4Glcb-Sp0 & 19 & 17 & 86 \\
\hline 588 & $\begin{array}{l}\text { GlcNAcb1-3Galb1-4GlcNAcb1-3Galb1-4GlcNAcb1-3Galb1-4GlcNAcb1-3Galb1-4GlcNAcb1-6(GlcNAcb1-3Galb1- } \\
\text { 4GlcNAcb1-3Galb1-4GlcNAcb1-3Galb1-4GlcNAcb1-3Galb1-4GIcNAb1-2)Mana1-6(GlcNAcb1-3Galb1- } \\
\text { 4GlcNAcb1-3Galb1-4GlcNAcb1-3Galb1-4GlcNAcb1-3Galb1-4GIcNAcb1-2Mana1-3)Manb1-4GlcNAcb1-4(Fuca1- } \\
\text { 6)GlcNAcb-Sp24 }\end{array}$ & 19 & 7 & 34 \\
\hline 241 & Neu5Aca2-3Galb1-3(Fuca1-4)GlcNAcb1-3Galb1-4(Fuca1-3)GIcNAcb-Sp0 & 19 & 5 & 25 \\
\hline 566 & $\begin{array}{l}\text { Galb1-4GlcNAcb1-3Galb1-4GIcNAcb1-3Galb1-4GIcNAcb1-3Galb1-4GlcNAcb1-3Galb1-4GlcNAcb1-2Mana1- } \\
\text { 6(Galb1-4GlcNAcb1-3Galb1-4GlcNAcb1-3Galb1-4GlcNAcb1-3Galb1-4GlcNAcb1-3Galb1-4GlcNAcb1-2Mana1- } \\
\text { 3)Manb1-4GlcNAcb1-4GlcNAcb-Sp25 }\end{array}$ & 19 & 25 & 129 \\
\hline 400 & Neu5Aca2-3Galb1-3GlcNAcb1-3GalNAca-Sp14 & 19 & 15 & 76 \\
\hline 435 & $\begin{array}{l}\text { GlcNAcb1-6(GlcNAcb1-2)Mana1-6(GlcNAcb1-4)(GlcNAcb1-4(GlcNAcb1-2)Mana1-3)Manb1-4GIcNAcb1- } \\
\text { 4GIcNAc-Sp21 }\end{array}$ & 19 & 6 & 31 \\
\hline 593 & Neu5Aca2-3Galb1-4GIcNAcb1-3Galb1-4GIcNAcb1-3GalNAca-Sp14 & 19 & 8 & 40 \\
\hline 3 & Mana-Sp8 & 19 & 4 & 21 \\
\hline 296 & Neu5Aca2-3Galb1-4GIcNAcb1-3Galb1-3GIcNAcb-Sp0 & 19 & 12 & 60 \\
\hline 134 & GlcNAcb1-6(Galb1-3)GalNAca-Sp8 & 19 & 5 & 25 \\
\hline 13 & Glcb-Sp8 & 19 & 8 & 40 \\
\hline 286 & Neu5Gca2-6GalNAca-Sp0 & 19 & 21 & 111 \\
\hline 385 & Galb1-4GIcNAcb1-6(Fuca1-4(Fuca1-2Galb1-3)GIcNAcb1-3)Galb1-4GIc-Sp21 & 19 & 14 & 71 \\
\hline 440 & Galb1-4Galb-Sp10 & 19 & 3 & 17 \\
\hline 473 & $\begin{array}{l}\text { Fuca1-2Galb1-4(Fuca1-3)GIcNAcb1-2Mana1-6(Fuca1-2Galb1-4(Fuca1-3)GlcNAcb1-2Mana1-3)Manb1- } \\
\text { 4GIcNAcb1-4(Fuca1-6)GIcNAcb-Sp24 }\end{array}$ & 19 & 11 & 60 \\
\hline 568 & Galb1-3GIcNAcb1-6(Galb1-3)GalNAc-Sp14 & 19 & 5 & 28 \\
\hline 219 & Neu5Aca2-3Galb1-4GIcNAcb1-3Galb1-4(Fuca1-3)GIcNAcb-Sp0 & 19 & 9 & 48 \\
\hline 363 & $\begin{array}{l}\text { Fuca1-2Galb1-4(Fuca1-3)GlcNAcb1-2Mana1-6(Fuca1-2Galb1-4(Fuca1-3)GIcNAcb1-2Mana1-3)Manb1- } \\
\text { 4GIcNAcb1-4GlcNAb-Sp20 }\end{array}$ & 19 & 9 & 51 \\
\hline 456 & $\begin{array}{l}\text { Gala1-3(Fuca1-2)Galb1-3GIcNAcb1-2Mana1-6(Gala1-3(Fuca1-2)Galb1-3GlcNAcb1-2Mana1-3)Manb1- } \\
\text { 4GIcNAcb1-4(Fuca1-6)GlcNAcb-Sp22 }\end{array}$ & 19 & 6 & 30 \\
\hline 424 & Gala1-3(Fuca1-2)Galb1-3GIcNAcb1-3GalNAc-Sp14 & 19 & 6 & 33 \\
\hline 583 & $\begin{array}{l}\text { Galb1-4GIcNAcb1-3Galb1-4GIcNAcb1-6(Galb1-4GIcNAcb1-3Galb1-4GlcNAb1-2)Mana1-6(Galb1-4GIcNAcb1- } \\
\text { 3Galb1-4GIcNAcb1-2Mana1-3)Manb1-4GIcNAcb1-4(Fuca1-6)GIcNAcb-Sp24 }\end{array}$ & 18 & 5 & 25 \\
\hline 376 & $\begin{array}{l}\text { Fuca1-4(Fuca1-2Galb1-3)GıNAcb1-2Mana1-3(Fuca1-4(Fuca1-2Galb1-3)GlcNAcb1-2Mana1-3)Manb1- } \\
\text { 4GIcNAcb1-4GIcNAcb-Sp19 }\end{array}$ & 18 & 11 & 61 \\
\hline 428 & $\begin{array}{l}\text { Gala1-3(Fuca1-2)Galb1-4GıNAcb1-2Mana1-6(Gala1-3(Fuca1-2)Galb1-4GıNAcb1-2Mana1-3)Manb1- } \\
\text { 4GlcNAcb1-4(Fuca1-6)GlcNAcb-Sp22 }\end{array}$ & 18 & 8 & 46 \\
\hline 65 & Fuca1-2Galb1-3GalNAcb1-4(Neu5Aca2-3)Galb1-4Glcb-Sp9 & 18 & 6 & 31 \\
\hline 523 & Gala1-3(Fuca1-2)Galb1-4GIcNAcb1-2Mana-Sp0 & 18 & 7 & 39 \\
\hline 582 & $\begin{array}{l}\text { Galb1-4GIcNAcb1-3Galb1-4GIcNAcb1-3Galb1-4GIcNAcb1-3Galb1-4GlcNAcb1-3Galb1-4GlcNAcb1-2Mana1- } \\
\text { 6(Galb1-4GlcNAcb1-3Galb1-4GlcNAcb1-3Galb1-4GlcNAcb1-3Galb1-4GIcNAcb1-3Galb1-4GIcNAcb1-2Mana1- } \\
\text { 3)Manb1-4GlcNAcb1-4(Fuca1-6)GlcNAcb-Sp19 }\end{array}$ & 18 & 6 & 32 \\
\hline 203 & GlcAb-Sp8 & 18 & 16 & 86 \\
\hline
\end{tabular}




\begin{tabular}{|c|c|c|c|c|}
\hline 304 & GlcNAcb1-6(Galb1-4GIcNAcb1-3)Galb1-4GIcNAc-Sp0 & 18 & 7 & 39 \\
\hline 355 & Galb1-3GIcNAcb1-2Mana1-6(Galb1-3GlcNAcb1-2Mana1-3)Manb1-4GIcNAcb1-4(Fuca1-6)GIcNAcb-Sp22 & 18 & 14 & 78 \\
\hline 387 & Galb1-3GIcNAcb1-3Galb1-4(Fuca1-3)GlcNAcb1-6(Galb1-3GlcNAcb1-3)Galb1-4Glc-Sp21 & 18 & 10 & 58 \\
\hline 495 & Fuca1-2Galb1-4GIcNAcb1-6GalNAca-Sp14 & 18 & 5 & 29 \\
\hline 310 & GlcNAcb1-3Man-Sp10 & 18 & 8 & 47 \\
\hline 502 & Fuca1-2(6S)Galb1-3(6S)GlcNAcb-Sp0 & 18 & 6 & 31 \\
\hline 498 & Fuca1-2(6S)Galb1-3GlcNAcb-Sp0 & 18 & 13 & 71 \\
\hline 312 & GlcNAcb1-4GIcNAcb-Sp12 & 17 & 5 & 27 \\
\hline 213 & Mana1-2Mana1-6(Mana1-2Mana1-3)Mana1-6(Mana1-2Mana1-2Mana1-3)Manb1-4GIcNAcb1-4GIcNAcb-Sp12 & 17 & 6 & 32 \\
\hline 359 & KDNa2-3Galb1-4Glc-Sp0 & 17 & 12 & 69 \\
\hline 574 & Neu5Aca2-8Neu5Aca2-3Galb1-3GalNAcb1-4(Neu5Aca2-3)Galb1-4Glc-Sp21 & 17 & 6 & 33 \\
\hline 278 & Galb1-3(Fuca1-4)GlcNAcb1-3Galb1-3(Fuca1-4)GlcNAcb-Sp0 & 17 & 14 & 81 \\
\hline 449 & Galb1-4GlcNAcb1-2Mana-Sp0 & 17 & 10 & 60 \\
\hline 439 & $\begin{array}{l}\text { Galb1-4GlcNAcb1-6(Galb1-4GlcNAcb1-2)Mana1-6(GlcNAcb1-4)(Galb1-4GIcNAcb1-4(Galb1-4GIcNAcb1- } \\
\text { 2)Mana1-3)Manb1-4GlcNAcb1-4GIcNAc-Sp21 }\end{array}$ & 17 & 8 & 46 \\
\hline 268 & Neu5Aca2-6Galb1-4(6S)GlcNAcb-Sp8 & 17 & 4 & 26 \\
\hline 608 & $\begin{array}{l}\text { Neu5Aca2-3Galb1-4GIcNAcb1-3Galb1-4GIcNAcb1-3Galb1-4GlcNAcb1-2Mana1-6(Neu5Aca2-3Galb1- } \\
\text { 4GlcNAcb1-3Galb1-4GlcNAcb1-3Galb1-4GlcNAcb1-2Mana1-3)Manb1-4GIcNAcb1-4GlcNAcb-Sp12 }\end{array}$ & 17 & 7 & 43 \\
\hline 169 & Galb1-4GIcNAcb-Sp0 & 17 & 9 & 54 \\
\hline 403 & GalNAca1-3GalNAcb1-3Gala1-4Galb1-4GIcNAcb-Sp0 & 17 & 3 & 20 \\
\hline 206 & KDNa2-3Galb1-3GlcNAcb-Sp0 & 17 & 9 & 54 \\
\hline 575 & $\begin{array}{l}\text { GlcNAcb1-3Galb1-4GlcNAcb1-2Mana1-6(GlcNAcb1-3Galb1-4GlcNAcb1-2Mana1-3)Manb1-4GlcNAcb1-4(Fuca1- } \\
\text { 6)GlcNAcb-Sp24 }\end{array}$ & 17 & 7 & 42 \\
\hline 599 & GlcNAcb1-3Galb1-4GlcNAcb1-3Galb1-4GlcNAcb1-3GalNAca-Sp14 & 17 & 10 & 59 \\
\hline 122 & Gala1-4Galb1-4GIcNAcb-Sp8 & 17 & 1 & 6 \\
\hline 11 & Neu5Acb-Sp8 & 17 & 9 & 51 \\
\hline 184 & GlcNAcb1-3Galb1-4GlcNAcb-Sp8 & 17 & 6 & 37 \\
\hline 174 & GlcNAca1-3Galb1-4GIcNAcb-Sp8 & 17 & 12 & 73 \\
\hline 370 & $\begin{array}{l}\text { Galb1-4GIcNAcb1-2Mana1-6(Galb1-4GlcNAcb1-4(Galb1-4GIcNAcb1-2)Mana1-3)Manb1-4GlcNAcb1-4GlcNAc- } \\
\text { Sp21 }\end{array}$ & 17 & 8 & 50 \\
\hline 52 & GlcNAcb1-2Mana1-6(GlcNAcb1-2Mana1-3)Manb1-4GlcNAcb1-4GlcNAcb-Sp12 & 17 & 11 & 69 \\
\hline 507 & GalNAca1-3(Fuca1-2)Galb1-3GIcNAcb1-6GalNAca-Sp14 & 17 & 9 & 57 \\
\hline 257 & Neu5Aca2-3Galb1-4(Fuca1-3)GIcNAcb1-3Galb-Sp8 & 16 & 16 & 97 \\
\hline 103 & Gala1-3(Fuca1-2)Galb1-3GlcNAcb-Sp8 & 16 & 4 & 22 \\
\hline 550 & $\begin{array}{l}\text { GlcNAcb1-3Galb1-4GlcNAcb1-3Galb1-4GlcNAcb1-3Galb1-4GlcNAcb1-2Mana1-6(GlcNAcb1-3Galb1-4GlcNAcb1- } \\
\text { 3Galb1-4GlcNAcb1-3Galb1-4GIcNAcb1-2Mana1-3)Manb1-4GlcNAcb1-4GlcNAcb-Sp25 }\end{array}$ & 16 & 8 & 49 \\
\hline 528 & Gala1-3Galb1-3GlcNAcb1-2Mana-Sp0 & 16 & 10 & 63 \\
\hline 168 & Galb1-4GIcNAcb1-6(Galb1-3)GalNAc-Sp14 & 16 & 19 & 116 \\
\hline 24 & (3S)Galb1-4(Fuca1-3)(6S)Glc-Sp0 & 16 & 15 & 91 \\
\hline 600 & Galb1-4GIcNAcb1-3Galb1-3GalNAca-Sp14 & 16 & 7 & 43 \\
\hline 530 & Neu5Aca2-3Galb1-3GlcNAcb1-4Galb1-4GIcb-Sp0 & 16 & 6 & 40 \\
\hline 142 & Galb1-3GalNAca-Sp16 & 16 & 16 & 99 \\
\hline 291 & Galb1-4(Fuca1-3)(6S)GIcNAcb-Sp0 & 16 & 9 & 59 \\
\hline
\end{tabular}




\begin{tabular}{|c|c|c|c|c|}
\hline 82 & Fucb1-3GlcNAcb-Sp8 & 16 & 2 & 12 \\
\hline 246 & Neu5Aca2-3Galb-Sp8 & 16 & 22 & 133 \\
\hline 534 & Fuca1-4(Galb1-3)GlcNAcb1-2 Mana-Sp0 & 16 & 8 & 47 \\
\hline 503 & Neu5Aca2-6GalNAcb1-4(6S)GlcNAcb-Sp8 & 16 & 14 & 88 \\
\hline 159 & Galb1-4GalNAcb1-3(Fuca1-2)Galb1-4GlcNAcb-Sp8 & 16 & 13 & 78 \\
\hline 596 & GIcNAcb1-3Galb1-4GIcNAcb1-6(GIcNAcb1-3Galb1-4GIcNAcb1-3)GalNAca-Sp14 & 16 & 4 & 28 \\
\hline 389 & GlcNAcb1-2Mana1-6(GlcNAcb1-4(GlcNAcb1-2)Mana1-3)Manb1-4GlcNAcb1-4GIcNAc-Sp21 & 16 & 7 & 41 \\
\hline 544 & $\begin{array}{l}\text { Neu5Gca2-3Galb1-4GlcNAcb1-3Galb1-4GlcNAcb1-2Mana1-6(Neu5Gca2-3Galb1-4GlcNAcb1-3Galb1- } \\
\text { 4GlcNAcb1-2Mana1-3)Manb1-4GlcNAcb1-4GlcNAcb-Sp24 }\end{array}$ & 16 & 4 & 27 \\
\hline 390 & Fuca1-2Galb1-3GalNAca1-3(Fuca1-2)Galb1-4Glcb-Sp0 & 16 & 13 & 82 \\
\hline 607 & $\begin{array}{l}\text { Neu5Aca2-6Galb1-4GlcNAcb1-3Galb1-4GlcNAcb1-3Galb1-4GlcNAcb1-2Mana1-6(Neu5Aca2-6Galb1- } \\
\text { 4GlcNAcb1-3Galb1-4GIcNAcb1-3Galb1-4GlcNAcb1-2Mana1-3)Manb1-4GIcNAcb1-4GIcNAcb-Sp12 }\end{array}$ & 16 & 8 & 52 \\
\hline 90 & GalNAca1-3(Fuca1-2)Galb-Sp8 & 16 & 10 & 61 \\
\hline 42 & (6S)Galb1-4GIcb-Sp0 & 16 & 11 & 69 \\
\hline 101 & Gala1-2Galb-Sp8 & 16 & 15 & 98 \\
\hline 133 & Galb1-4GIcNAcb1-6GalNAc-Sp14 & 16 & 19 & 120 \\
\hline 572 & $\begin{array}{l}\text { Galb1-3GlcNAcb1-3Galb1-4GIcNAcb1-3Galb1-4GIcNAcb1-6(Galb1-3GlcNAcb1-3Galb1-4GIcNAcb1-3Galb1- } \\
\text { 4GIcNAb1-2)Mana1-6(Galb1-3GIcNAcb1-3Galb1-4GIcNAcb1-3Galb1-4GIcNAcb1-2Mana1-3)Manb1-4GIcNAcb1- } \\
\text { 4(Fuca1-6)GIcNAcb-Sp24 }\end{array}$ & 16 & 7 & 43 \\
\hline 560 & $\begin{array}{l}\text { Galb1-4GIcNAcb1-3Galb1-4GIcNAcb1-6(Galb1-4GIcNAcb1-3Galb1-4GIcNAcb1-2)Mana1-6(Galb1-4GIcNAcb1- } \\
\text { 3Galb1-4GlcNAcb1-2Mana1-3)Mana1-4GIcNAcb1-4GIcNAc-Sp24 }\end{array}$ & 16 & 10 & 61 \\
\hline 446 & $\begin{array}{l}\text { Fuca1-2Galb1-4 GIcNAcb1-2Mana1-6(Fuca1-2Galb1-4GIcNAcb1-2(Fuca1-2Galb1-4GIcNAcb1-4)Mana1- } \\
\text { 3)Manb1-4GIcNAcb1-4GIcNAcb-Sp12 }\end{array}$ & 15 & 12 & 77 \\
\hline 350 & Galb1-4GIcNAcb1-2Mana1-3Manb1-4GIcNAcb1-4GIcNAc-Sp12 & 15 & 9 & 58 \\
\hline 519 & (6P)Galb1-4GlcNAcb-SP0 & 15 & 5 & 31 \\
\hline 223 & Fuca1-2(6S)Galb1-4(6S)Glcb-Sp0 & 15 & 7 & 46 \\
\hline 360 & KDNa2-3Galb1-3GalNAca-Sp14 & 15 & 4 & 28 \\
\hline 33 & (3S)Galb1-4(Fuca1-3)GlcNAc-Sp8 & 15 & 11 & 74 \\
\hline 68 & Fuca1-2Galb1-3GIcNAcb-Sp0 & 15 & 12 & 79 \\
\hline 94 & GalNAca1-4(Fuca1-2)Galb1-4GlcNAcb-Sp8 & 15 & 11 & 75 \\
\hline 139 & Neu5Aca2-6(Galb1-3)GlcNAcb1-4Galb1-4Glcb-Sp10 & 15 & 4 & 30 \\
\hline 48 & $\mathrm{Neu} 5,9 \mathrm{Ac}_{2} \mathrm{a}-\mathrm{Sp} 8$ & 15 & 9 & 59 \\
\hline 10 & Neu5Aca-Sp11 & 15 & 15 & 103 \\
\hline 551 & $\begin{array}{l}\text { Galb1-4GIcNAcb1-3Galb1-4GlcNAcb1-3Galb1-4GlcNAcb1-3Galb1-4GlcNAcb1-2Mana1-6(Galb1-4GlcNAcb1- } \\
\text { 3Galb1-4GIcNAcb1-3Galb1-4GlcNAcb1-3Galb1-4GlcNAcb1-2Mana1-3)Manb1-4GlcNAcb1-4GlcNAcb-Sp25 }\end{array}$ & 15 & 9 & 61 \\
\hline 222 & Fuca1-2Galb1-4(6S)GlcNAcb-Sp8 & 15 & 22 & 148 \\
\hline 55 & $\begin{array}{l}\text { Neu5Aca2-6Galb1-4GlcNAcb1-2Mana1-6(Neu5Aca2-6Galb1-4GlcNAcb1-2Mana1-3)Manb1-4GIcNAcb1- } \\
\text { 4GlcNAcb-Sp12 }\end{array}$ & 15 & 15 & 99 \\
\hline 514 & (3S)GalNAcb1-4(3S)GlcNAc-Sp8 & 15 & 6 & 41 \\
\hline 124 & Gala1-4GlcNAcb-Sp8 & 15 & 13 & 84 \\
\hline 576 & $\begin{array}{l}\text { Galb1-4GIcNAcb1-3Galb1-4GlcNAcb1-2Mana1-6(Galb1-4GlcNAcb1-3Galb1-4GlcNAcb1-2Mana1-3)Manb1- } \\
\text { 4GlcNAcb1-4(Fuca1-6)GlcNAcb-Sp24 }\end{array}$ & 15 & 9 & 63 \\
\hline 469 & Gala1-3(Fuca1-2)Galb1-3GalNAcb-Sp8 & 15 & 9 & 63 \\
\hline
\end{tabular}




\begin{tabular}{|c|c|c|c|c|}
\hline 430 & Galb1-4GlcNAcb1-6(Fuca1-2Galb1-3GlcNAcb1-3)Galb1-4Glc-Sp21 & 15 & 10 & 65 \\
\hline 354 & Galb1-4GlcNAcb1-2Mana1-6(Galb1-4GlcNAcb1-2Mana1-3)Manb1-4GlcNAcb1-4(Fuca1-6)GlcNAcb-Sp22 & 15 & 14 & 98 \\
\hline 522 & Gala1-3Galb1-4GlcNAcb1-2Mana-Sp0 & 15 & 7 & 49 \\
\hline 395 & Gala1-3Galb1-3GlcNAcb1-2Mana1-6(Gala1-3Galb1-3GlcNAcb1-2Mana1-3)Manb1-4GIcNAcb1-4GIcNAc-Sp19 & 15 & 8 & 57 \\
\hline 331 & Neu5Aca2-6Galb1-4GlcNAcb1-3Galb1-3GlcNAcb-Sp0 & 15 & 7 & 49 \\
\hline 277 & Neu5Aca2-8Neu5Aca2-3Galb1-4Glcb-Sp0 & 15 & 11 & 75 \\
\hline 152 & Galb1-4(Fuca1-3)GlcNAcb-Sp0 & 15 & 15 & 100 \\
\hline 306 & Galb1-4GIcNAcb1-6Galb1-4GIcNAcb-Sp0 & 15 & 8 & 53 \\
\hline 461 & $\begin{array}{l}\text { Neu5Aca2-3Galb1-4GlcNAcb1-4Mana1-6(GlcNAcb1-4)(Neu5Aca2-3Galb1-4GlcNAcb1-4(Neu5Aca2-3Galb1- } \\
\text { 4GlcNAcb1-2)Mana1-3)Manb1-4GlcNAcb1-4GlcNAcb-Sp21 }\end{array}$ & 14 & 7 & 48 \\
\hline 397 & $\begin{array}{l}\text { Neu5Aca2-3Galb1-3GlcNAcb1-2Mana1-6(Neu5Aca2-3Galb1-3GlcNAcb1-2Mana1-3)Manb1-4GIcNAcb1- } \\
\text { 4GIcNAc-Sp19 }\end{array}$ & 14 & 5 & 35 \\
\hline 17 & GlcNAcb-Sp8 & 14 & 12 & 82 \\
\hline 590 & Galb1-4GIcNAcb1-3Galb1-4GIcNAcb1-3GalNAca-Sp14 & 14 & 5 & 33 \\
\hline 119 & Gala1-3Galb-Sp8 & 14 & 7 & 51 \\
\hline 470 & Glca1-6Glca1-6Glca1-6Glcb-Sp10 & 14 & 14 & 100 \\
\hline 108 & Gala1-3(Fuca1-2)Galb-Sp8 & 14 & 2 & 14 \\
\hline 442 & Neu5Aca2-3Galb1-4GIcNAcb1-3Galb-Sp8 & 14 & 4 & 29 \\
\hline 451 & Gala1-3(Fuca1-2)Galb1-4GIcNAcb1-6(Gala1-3(Fuca1-2)Galb1-4GIcNAcb1-3)GalNAc-Sp14 & 14 & 15 & 103 \\
\hline 509 & $\begin{array}{l}\text { Galb1-4GIcNAcb1-6(Galb1-4GIcNAcb1-2)Mana1-6(GIcNAcb1-4)Galb1-4GIcNAcb1-4(Gal b1-4GIcNAcb1- } \\
\text { 2)Mana1-3)Manb1-4GIcNAcb1-4(Fuca1-6)GIcNAc-Sp21 }\end{array}$ & 14 & 9 & 63 \\
\hline 104 & Gala1-3(Fuca1-2)Galb1-4(Fuca1-3)GIcNAcb-Sp0 & 14 & 4 & 31 \\
\hline 492 & Neu5Aca2-3Galb1-3GIcNAcb1-6GalNAca-Sp14 & 14 & 4 & 26 \\
\hline 316 & Mana1-2Mana1-6(Mana1-3)Mana1-6(Mana1-2Mana1-2Mana1-3)Mana-Sp9 & 14 & 2 & 12 \\
\hline 501 & Fuca1-2Galb1-3(6S)GIcNAcb-Sp0 & 14 & 11 & 79 \\
\hline 145 & Galb1-3GalNAcb1-4(Neu5Aca2-3)Galb1-4GIcb-Sp0 & 14 & 5 & 32 \\
\hline 339 & GlcNAca1-4Galb1-4GlcNAcb1-3Galb1-4GlcNAcb1-3Galb1-4GIcNAcb-Sp0 & 14 & 13 & 90 \\
\hline 416 & GalNAca1-3GalNAcb1-3Gala1-4Galb1-4Glcb-Sp0 & 14 & 8 & 55 \\
\hline 173 & Galb1-4GIcb-Sp8 & 14 & 10 & 70 \\
\hline 592 & Galb1-4GIcNAcb1-3Galb1-4GIcNAcb1-6(Galb1-4GlcNAcb1-3Galb1-4GlcNAcb1-3)GalNAca-Sp14 & 14 & 4 & 27 \\
\hline 371 & $\begin{array}{l}\text { GalNAca1-3(Fuca1-2)Galb1-4GIcNAcb1-2Mana1-6(GalNAca1-3(Fuca1-2)Galb1-4GlcNAcb1-2Mana1-3)Manb1- } \\
\text { 4GlcNAcb1-4GlcNAcb-Sp20 }\end{array}$ & 14 & 9 & 66 \\
\hline 102 & Gala1-3(Fuca1-2)Galb1-3GIcNAcb-Sp0 & 14 & 14 & 105 \\
\hline 513 & (6S)GalNAcb1-4GIcNAc-Sp8 & 14 & 7 & 52 \\
\hline 251 & Neu5Aca2-3Galb1-3GIcNAcb-Sp8 & 14 & 7 & 52 \\
\hline 34 & (3S)Galb1-4(6S)GlcNAcb-Sp0 & 14 & 12 & 86 \\
\hline 262 & Neu5Aca2-3Galb1-4GlcNAcb1-3Galb1-4GlcNAcb-Sp0 & 14 & 6 & 46 \\
\hline 402 & Galb1-4(Fuca1-3)GlcNAcb1-3GalNAca-Sp14 & 14 & 9 & 66 \\
\hline 167 & Galb1-4GIcNAcb1-6(Galb1-3)GalNAca-Sp8 & 14 & 5 & 33 \\
\hline 67 & Fuca1-2Galb1-3GIcNAcb1-3Galb1-4Glcb-Sp10 & 14 & 9 & 65 \\
\hline 437 & $\begin{array}{l}\text { Galb1-4GlcNAcb1-2Mana1-6(GlcNAcb1-4)(Galb1-4GlcNAcb1-4(Galb1-4GlcNAcb1-2)Mana1-3)Manb1- } \\
\text { 4GlcNAcb1-4GlcNAc-Sp21 }\end{array}$ & 14 & 18 & 134 \\
\hline 601 & Neu5Aca2-3Galb1-4GlcNAcb1-3Galb1-4GlcNAcb1-6(Galb1-3)GalNAca-Sp14 & 14 & 3 & 22 \\
\hline
\end{tabular}




\begin{tabular}{|c|c|c|c|c|}
\hline 438 & $\begin{array}{l}\text { Galb1-4GIcNAcb1-6(Galb1-4GIcNAcb1-2)Mana1-6(GlcNAcb1-4)(Galb1-4GlcNAcb1-2Mana1-3)Manb1- } \\
\text { 4GlcNAcb1-4GIcNAc-Sp21 }\end{array}$ & 14 & 12 & 86 \\
\hline 314 & Mana1-6Manb-Sp10 & 14 & 9 & 67 \\
\hline 235 & Neu5Aca2-3Galb1-3GalNAcb1-4(Neu5Aca2-3)Galb1-4Glcb-Sp0 & 14 & 10 & 76 \\
\hline 176 & GlcNAcb1-2Galb1-3GalNAca-Sp8 & 13 & 16 & 120 \\
\hline 394 & GalNAca1-3(Fuca1-2)Galb1-3GalNAca1-3(Fuca1-2)Galb1-4GIcNAcb-Sp0 & 13 & 5 & 35 \\
\hline 441 & Galb1-6Galb-Sp10 & 13 & 4 & 27 \\
\hline 420 & $\begin{array}{l}\text { Galb1-4(Fuca1-3)GlcNAcb1-2Mana1-6(Galb1-4(Fuca1-3)GlcNAcb1-2Mana1-3)Manb1-4GIcNAcb1-4(Fuca1- } \\
\text { 6)GlcNAcb-Sp22 }\end{array}$ & 13 & 4 & 26 \\
\hline 448 & Galb1-4(Fuca1-3)GlcNAcb1-6GalNAc-Sp14 & 13 & 14 & 104 \\
\hline 478 & Neu5Aca2-6Galb1-4GIcNAcb1-6(Galb1-3GIcNAcb1-3)Galb1-4Glcb-Sp21 & 13 & 18 & 137 \\
\hline 274 & Neu5Aca2-6Galb1-4GIcb-Sp8 & 13 & 14 & 103 \\
\hline 91 & GalNAca1-3(Fuca1-2)Galb-Sp18 & 13 & 18 & 138 \\
\hline 28 & (3S)Galb1-3(Fuca1-4)GlcNAcb-Sp8 & 13 & 7 & 52 \\
\hline 546 & $\begin{array}{l}\text { GlcNAcb1-3Galb1-4GlcNAcb1-3Galb1-4GlcNAcb1-2Mana1-6(GlcNAcb1-3Galb1-4GlcNAcb1-3Galb1-4GlcNAcb1- } \\
\text { 2Mana1-3)Manb1-4GlcNAcb1-4GlcNAcb-Sp12 }\end{array}$ & 13 & 6 & 42 \\
\hline 279 & Neu5Acb2-6GalNAca-Sp8 & 13 & 3 & 23 \\
\hline 226 & GalNAcb1-4(Neu5Aca2-8Neu5Aca2-8Neu5Aca2-8Neu5Aca2-3)Galb1-4Glcb-Sp0 & 13 & 12 & 93 \\
\hline 567 & GIcNAb1-3Galb1-3GalNAc-Sp14 & 13 & 14 & 105 \\
\hline 215 & Mana1-2Mana1-2Mana1-6(Mana1-3)Mana-Sp9 & 13 & 10 & 73 \\
\hline 597 & $\begin{array}{l}\text { Neu5Aca2-3Galb1-4GIcNAcb1-3Galb1-4GIcNAcb1-6(Neu5Aca2-3Galb1-4GIcNAcb1-3Galb1-4GIcNAcb1- } \\
\text { 3)GalNAca-Sp14 }\end{array}$ & 13 & 7 & 52 \\
\hline 398 & GlcNAcb1-2Mana1-6(Galb1-4GlcNAcb1-2Mana1-3)Manb1-4GlcNAcb1-4GlcNAc-Sp12 & 13 & 9 & 71 \\
\hline 121 & Gala1-4Galb1-4GlcNAcb-Sp0 & 13 & 15 & 113 \\
\hline 260 & Neu5Aca2-3Galb1-4GIcNAcb-Sp0 & 13 & 3 & 22 \\
\hline 584 & $\begin{array}{l}\text { GlcNAcb1-3Galb1-4GlcNAcb1-3Galb1-4GIcNAcb1-6(GlcNAcb1-3Galb1-4GIcNAcb1-3Galb1-4GIcNAb1-2)Mana1- } \\
\text { 6(GlcNAcb1-3Galb1-4GIcNAcb1-3Galb1-4GIcNAcb1-2Mana1-3)Manb1-4GlcNAcb1-4(Fuca1-6)GlcNAcb-Sp24 }\end{array}$ & 13 & 9 & 68 \\
\hline 300 & Neu5Aca2-3Galb1-4(Fuca1-3)GlcNAcb1-6(Galb1-3)GalNAca-Sp14 & 13 & 4 & 28 \\
\hline 117 & Gala1-3Galb1-4GIcb-Sp0 & 13 & 4 & 30 \\
\hline 462 & $\begin{array}{l}\text { Neu5Aca2-3Galb1-4GIcNAcb1-6(Neu5Aca2-3Galb1-4GIcNAcb1-2)Mana1-6(GlcNAcb1-4)(Neu5Aca2-3Galb1- } \\
\text { 4GIcNAcb1-2Mana1-3)Manb1-4GIcNAcb1-4GIcNAcb-Sp21 }\end{array}$ & 13 & 8 & 59 \\
\hline 517 & (4S)GalNAcb-Sp10 & 13 & 6 & 50 \\
\hline 150 & Galb1-3GlcNAcb-Sp0 & 13 & 11 & 82 \\
\hline 26 & (3S)Galb1-4(6S)Glcb-Sp0 & 13 & 9 & 74 \\
\hline 147 & Galb1-3Galb-Sp8 & 13 & 11 & 84 \\
\hline 230 & Neu5Aca2-8Neu5Aca2-8Neu5Aca-Sp8 & 13 & 7 & 58 \\
\hline 318 & Neu5Aca2-3Galb1-4GIcNAcb1-6(Neu5Aca2-3Galb1-3)GalNAca-Sp14 & 13 & 11 & 90 \\
\hline 346 & Neu5Aca2-6Galb1-4GIcNAcb1-2Mana1-6(Mana1-3)Manb1-4GIcNAcb1-4GIcNAc-Sp12 & 13 & 9 & 71 \\
\hline 35 & (3S)Galb1-4(6S)GlcNAcb-Sp8 & 13 & 3 & 24 \\
\hline 399 & Galb1-4GIcNAcb1-2Mana1-6(GIcNAcb1-2Mana1-3)Manb1-4GIcNAcb1-4GIcNAc-Sp12 & 13 & 7 & 58 \\
\hline 158 & Galb1-4GalNAca1-3(Fuca1-2)Galb1-4GlcNAcb-Sp8 & 13 & 2 & 18 \\
\hline 552 & $\begin{array}{l}\text { Galb1-3GIcNAcb1-3Galb1-4GlcNAcb1-2Mana1-6(Galb1-3GIcNAcb1-3Galb1-4GIcNAcb1-2Mana1-3)Manb1- } \\
\text { 4GlcNAcb1-4GIcNAc-Sp25 }\end{array}$ & 13 & 12 & 94 \\
\hline
\end{tabular}




\begin{tabular}{|c|c|c|c|c|}
\hline 533 & $\begin{array}{l}\text { Galb1-4GIcNAcb1-2 Mana1-6(Galb1-4GIcNAcb1-4)(Galb1-4GIcNAcb1-2Mana1-3)Manb1-4GIcNAcb1-4(Fuca1- } \\
\text { 6)GIcNAc-Sp21 }\end{array}$ & 12 & 5 & 42 \\
\hline 401 & Fuca1-2Galb1-4GIcNAcb1-3GalNAca-Sp14 & 12 & 6 & 46 \\
\hline 233 & GalNAcb1-4(Neu5Aca2-3)Galb1-4GlcNAcb-Sp8 & 12 & 8 & 61 \\
\hline 77 & Fuca1-2Galb1-4GIcNAcb-Sp8 & 12 & 15 & 119 \\
\hline 543 & $\begin{array}{l}\text { Galb1-4GlcNAcb1-3Galb1-4GlcNAcb1-2Mana1-6(Galb1-4GlcNAcb1-3Galb1-4GlcNAcb1-2Mana1-3)Manb1- } \\
\text { 4GIcNAcb1-4GlcNAcb-Sp24 }\end{array}$ & 12 & 10 & 79 \\
\hline 272 & Neu5Aca2-6Galb1-4GIcNAcb1-3Galb1-4GIcNAcb-Sp0 & 12 & 4 & 32 \\
\hline 97 & GalNAcb1-3Gala1-4Galb1-4GIcNAcb-Sp0 & 12 & 7 & 56 \\
\hline 453 & Neu5Aca2-8Neu5Aca2-3Galb1-3GalNAcb1-4(Neu5Aca2-8Neu5Aca2-3)Galb1-4Glcb-Sp0 & 12 & 3 & 27 \\
\hline 377 & Neu5Aca2-3Galb1-4GIcNAcb1-3GalNAc-Sp14 & 12 & 6 & 45 \\
\hline 153 & Galb1-4(Fuca1-3)GIcNAcb-Sp8 & 12 & 8 & 69 \\
\hline 433 & GlcNAcb1-2Mana1-6(GlcNAcb1-4)(GlcNAcb1-4(GlcNAcb1-2)Mana1-3)Manb1-4GIcNAcb1-4GIcNAc-Sp21 & 12 & 8 & 64 \\
\hline 516 & (3S)GalNAcb1-4GlcNAc-Sp8 & 12 & 9 & 77 \\
\hline 479 & Neu5Aca2-3Galb1-4GlcNAcb1-2Mana-Sp0 & 12 & 4 & 35 \\
\hline 18 & $\mathrm{GlcN}(\mathrm{Gc}) \mathrm{b}-\mathrm{Sp} 8$ & 12 & 10 & 85 \\
\hline 490 & Gala1-3Galb1-3GlcNAcb1-6GalNAca-Sp14 & 12 & 8 & 69 \\
\hline 89 & GlcNAcb1-3Galb1-3GalNAca-Sp8 & 12 & 4 & 33 \\
\hline 362 & Fuca1-2Galb1-4GIcNAcb1-2Mana1-6(Fuca1-2Galb1-4GIcNAcb1-2Mana1-3)Manb1-4GIcNAcb1-4GIcNAcb-Sp20 & 12 & 7 & 60 \\
\hline 188 & GlcNAcb1-6(GIcNAcb1-4)GalNAca-Sp8 & 12 & 4 & 30 \\
\hline 125 & Gala1-6Glcb-Sp8 & 12 & 5 & 41 \\
\hline 327 & $\begin{array}{l}\text { Neu5Aca2-3Galb1-4GIcNAcb1-2Mana1-6(Neu5Aca2-6Galb1-4GlcNAcb1-2Mana1-3)Manb1-4GlcNAcb1- } \\
\text { 4GIcNAcb-Sp12 }\end{array}$ & 12 & 9 & 75 \\
\hline 333 & Neu5Aca2-6Galb1-4GIcNAcb1-3Galb1-4GIcNAcb1-3Galb1-4GIcNAcb-Sp0 & 12 & 10 & 81 \\
\hline 483 & $\begin{array}{l}\text { Neu5Aca2-6Galb1-4GIcNAcb1-2Mana1-6(Neu5Aca2-6Galb1-4GIcNAcb1-2Mana1-3)Manb1-4GIcNAcb1-4(Fuca1- } \\
\text { 6)GIcNAcb-Sp24 }\end{array}$ & 12 & 9 & 78 \\
\hline 382 & Galb1-3GalNAca1-3(Fuca1-2)Galb1-4GlcNAc-Sp0 & 12 & 6 & 50 \\
\hline 540 & $\begin{array}{l}\text { GlcNAcb1-3Galb1-4GIcNAcb1-2Mana1-6(GlcNAcb1-3Galb1-4GlcNAcb1-2Mana1-3)Manb1-4GIcNAcb1- } \\
\text { 4GlcNAcb-Sp12 }\end{array}$ & 12 & 3 & 29 \\
\hline 151 & Galb1-3GIcNAcb-Sp8 & 12 & 5 & 41 \\
\hline 538 & Gala1-3(Fuca1-2)Galb1-3GalNAcb1-3Gala1-4Galb1-4Glc-Sp21 & 12 & 7 & 58 \\
\hline 444 & (6S)Galb1-3GlcNAcb-Sp0 & 12 & 2 & 15 \\
\hline 73 & Fuca1-2Galb1-4(Fuca1-3)GlcNAcb-Sp8 & 12 & 4 & 32 \\
\hline 436 & Galb1-4GIcNAcb1-2Mana1-6(GIcNAcb1-4)(Galb1-4GIcNAcb1-2Mana1-3)Manb1-4GIcNAcb1-4GIcNAc-Sp21 & 12 & 14 & 117 \\
\hline 61 & Fuca1-2Galb1-3(Fuca1-4)GlcNAcb-Sp8 & 12 & 9 & 76 \\
\hline 301 & Galb1-3Galb1-4GlcNAcb-Sp8 & 12 & 2 & 21 \\
\hline 378 & Neu5Aca2-6Galb1-4GIcNAcb1-3GalNAc-Sp14 & 12 & 6 & 50 \\
\hline 106 & Gala1-3(Fuca1-2)Galb1-4GIcNAc-Sp0 & 12 & 11 & 91 \\
\hline 132 & Galb1-4GlcNAcb1-6GalNAca-Sp8 & 12 & 2 & 19 \\
\hline 105 & Gala1-3(Fuca1-2)Galb1-4(Fuca1-3)GIcNAcb-Sp8 & 12 & 7 & 62 \\
\hline 114 & Gala1-3Galb1-4(Fuca1-3)GıNAcb-Sp8 & 12 & 5 & 48 \\
\hline 41 & (6P)Mana-Sp8 & 11 & 10 & 88 \\
\hline 237 & Neu5Aca2-3GalNAca-Sp8 & 11 & 13 & 117 \\
\hline
\end{tabular}




\begin{tabular}{|c|c|c|c|c|}
\hline 93 & GalNAca1-3Galb-Sp8 & 11 & 4 & 32 \\
\hline 328 & $\begin{array}{l}\text { Galb1-4(Fuca1-3)GlcNAcb1-2Mana1-6(Galb1-4(Fuca1-3)GlcNAcb1-2Mana1-3)Manb1-4GlcNAcb1-4GlcNAcb- } \\
\text { Sp20 }\end{array}$ & 11 & 11 & 94 \\
\hline 157 & Galb1-4(6S)Glcb-Sp8 & 11 & 15 & 133 \\
\hline 604 & $\begin{array}{l}\text { Neu5Aca2-3Galb1-4GIcNAcb1-3Galb1-4GIcNAcb1-2Mana1-6(Neu5Aca2-3Galb1-4GlcNAcb1-3Galb1- } \\
\text { 4GIcNAcb1-2Mana1-3)Manb1-4GIcNAcb1-4GIcNAcb-Sp12 }\end{array}$ & 11 & 9 & 78 \\
\hline 418 & Gala1-3(Fuca1-2)Galb1-4(Fuca1-3)GlcNAcb1-3GalNAc-Sp14 & 11 & 13 & 119 \\
\hline 80 & Fuca1-3GIcNAcb-Sp8 & 11 & 2 & 16 \\
\hline 511 & Galb1-3(6S)GlcNAcb-Sp8 & 11 & 1 & 7 \\
\hline 71 & Fuca1-2Galb1-4(Fuca1-3)GlcNAcb1-3Galb1-4(Fuca1-3)GlcNAcb1-3Galb1-4(Fuca1-3)GlcNAcb-Sp0 & 11 & 9 & 80 \\
\hline 194 & GlcNAcb1-6GalNAca-Sp14 & 11 & 10 & 90 \\
\hline 47 & (6S)GIcNAcb-Sp8 & 11 & 4 & 38 \\
\hline 180 & GlcNAcb1-3GalNAca-Sp8 & 11 & 7 & 64 \\
\hline 39 & $(6 S)(4 S) G a l b 1-4 G I c N A c b-S p 0$ & 11 & 8 & 72 \\
\hline 9 & Neu5Aca-Sp8 & 11 & 7 & 67 \\
\hline 265 & Neu5Aca2-3Galb1-4GIcb-Sp8 & 11 & 10 & 89 \\
\hline 76 & Fuca1-2Galb1-4GIcNAcb-Sp0 & 11 & 7 & 64 \\
\hline 549 & $\begin{array}{l}\text { Galb1-4GlcNAcb1-3Galb1-4GlcNAcb1-3Galb1-4GlcNAcb1-2Mana1-6(Galb1-4GlcNAcb1-3Galb1-4GlcNAcb1- } \\
\text { 3Galb1-4GlcNAcb1-2Mana1-3)Manb1-4GlcNAcb1-4GlcNAcb-Sp24 }\end{array}$ & 11 & 7 & 68 \\
\hline 256 & Neu5Aca2-3Galb1-4(Fuca1-3)GlcNAcb-Sp8 & 11 & 3 & 30 \\
\hline 557 & Neu5Gca2-8Neu5Gca2-6Galb1-4GIcNAc-Sp0 & 11 & 4 & 41 \\
\hline 494 & Galb1-4(Fuca1-3)GIcNAcb1-6(Neu5Aca2-6(Neu5Aca2-3Galb1-3)GlcNAcb1-3)Galb1-4GIc-Sp21 & 11 & 7 & 64 \\
\hline 288 & Neu5Gca-Sp8 & 11 & 9 & 78 \\
\hline 302 & Neu5Aca2-6Galb1-4GIcNAcb1-2Mana1-6(Galb1-4GIcNAcb1-2Mana1-3)Manb1-4GIcNAcb1-4GIcNAcb-Sp12 & 11 & 11 & 98 \\
\hline 319 & $\begin{array}{l}\text { Neu5Aca2-6Galb1-4GIcNAcb1-2Mana1-6(Neu5Aca2-3Galb1-4GIcNAcb1-2Mana1-3)Manb1-4GIcNAcb1- } \\
\text { 4GIcNAcb-Sp12 }\end{array}$ & 11 & 4 & 36 \\
\hline 23 & 6S(3S)Galb1-4GlcNAcb-Sp0 & 11 & 2 & 22 \\
\hline 40 & (4S)Galb1-4GlcNAcb-Sp8 & 11 & 10 & 96 \\
\hline 225 & Neu5Aca2-3Galb1-3GalNAca-Sp14 & 11 & 4 & 35 \\
\hline 85 & (3S)Galb1-4(Fuca1-3)Glcb-Sp0 & 11 & 9 & 83 \\
\hline 602 & Neu5Aca2-6Galb1-4GIcNAcb1-3Galb1-4GlcNAcb1-6(Galb1-3)GalNAca-Sp14 & 11 & 4 & 33 \\
\hline 201 & G-ol-Sp8 & 11 & 8 & 77 \\
\hline 443 & GalNAcb1-6GalNAcb-Sp8 & 11 & 13 & 126 \\
\hline 535 & Neu5Aca2-3Galb1-4(Fuca1-3)GlcNAcb1-2Mana-Sp0 & 11 & 9 & 89 \\
\hline 25 & (3S)Galb1-4Glcb-Sp8 & 11 & 9 & 85 \\
\hline 197 & Glca1-4Glca-Sp8 & 10 & 8 & 79 \\
\hline 374 & $\begin{array}{l}\text { GalNAca1-3(Fuca1-2)Galb1-3GlcNAcb1-2Mana1-6(GalNAca1-3(Fuca1-2)Galb1-3GlcNAcb1-2Mana1-3)Manb1- } \\
\text { 4GIcNAcb1-4GIcNAcb-Sp20 }\end{array}$ & 10 & 3 & 27 \\
\hline 431 & Fuca1-3GlcNAcb1-6(Galb1-4GlcNAcb1-3)Galb1-4Glc-Sp21 & 10 & 12 & 115 \\
\hline 271 & Neu5Aca2-6Galb1-4GIcNAcb1-3Galb1-4(Fuca1-3)GlcNAcb1-3Galb1-4(Fuca1-3)GlcNAcb-Sp0 & 10 & 7 & 65 \\
\hline 239 & Neu5Aca2-3Galb1-3(6S)GlcNAc-Sp8 & 10 & 3 & 31 \\
\hline 252 & Neu5Aca2-3Galb1-4(6S)GlcNAcb-Sp8 & 10 & 4 & 43 \\
\hline 22 & 6S(3S)Galb1-4(6S)GlcNAcb-Sp0 & 10 & 9 & 88 \\
\hline
\end{tabular}




\begin{tabular}{|c|c|c|c|c|}
\hline 352 & Mana1-6(Galb1-4GlcNAcb1-2Mana1-3)Manb1-4GlcNAcb1-4GlcNAcb-Sp12 & 10 & 11 & 105 \\
\hline 69 & Fuca1-2Galb1-3GIcNAcb-Sp8 & 10 & 7 & 70 \\
\hline 21 & GlcNAcb1-6(GlcNAcb1-4)(GlcNAcb1-3)GlcNAc-Sp8 & 10 & 8 & 76 \\
\hline 524 & GalNAca1-3(Fuca1-2)Galb1-4 GIcNAcb1-2Mana-Sp0 & 10 & 6 & 55 \\
\hline 357 & KDNa2-3Galb1-4(Fuca1-3)GıNAc-Sp0 & 10 & 4 & 37 \\
\hline 43 & (6S)Galb1-4Glcb-Sp8 & 10 & 12 & 114 \\
\hline 6 & Fuca-Sp8 & 10 & 19 & 187 \\
\hline 253 & Neu5Aca2-3Galb1-4(Fuca1-3)(6S)GlcNAcb-Sp8 & 10 & 8 & 78 \\
\hline 155 & Galb1-4(Fuca1-3)GIcNAcb1-3Galb1-4(Fuca1-3)GIcNAcb1-3Galb1-4(Fuca1-3)GIcNAcb-Sp0 & 10 & 5 & 45 \\
\hline 154 & Galb1-4(Fuca1-3)GlcNAcb1-3Galb1-4(Fuca1-3)GlcNAcb-Sp0 & 10 & 4 & 42 \\
\hline 605 & GlcNAcb1-6(Neu5Aca2-3Galb1-3)GalNAca-Sp14 & 10 & 10 & 95 \\
\hline 373 & $\begin{array}{l}\text { Gala1-3Galb1-4(Fuca1-3)GlcNAcb1-2Mana1-6(Gala1-3Galb1-4(Fuca1-3)GlcNAcb1-2Mana1-3)Manb1- } \\
\text { 4GIcNAcb1-4GlcNAcb-Sp20 }\end{array}$ & 10 & 5 & 54 \\
\hline 19 & Galb1-4GIcNAcb1-6(Galb1-4GIcNAcb1-3)GalNAca-Sp8 & 10 & 6 & 59 \\
\hline 609 & $\begin{array}{l}\text { Neu5Aca2-6Galb1-4GIcNAcb1-3Galb1-4GIcNAcb1-2Mana1-6(Neu5Aca2-6Galb1-4GlcNAcb1-3Galb1- } \\
\text { 4GIcNAcb1-2Mana1-3)Manb1-4GIcNAcb1-4GIcNAcb-Sp12 }\end{array}$ & 10 & 5 & 54 \\
\hline 547 & $\begin{array}{l}\text { GlcNAcb1-3Galb1-4GIcNAcb1-3Galb1-4GlcNAcb1-2Mana1-6(GIcNAcb1-3Galb1-4GIcNAcb1-3Galb1-4GIcNAcb1- } \\
\text { 2Mana1-3)Manb1-4GIcNAcb1-4GlcNAcb-Sp25 }\end{array}$ & 10 & 3 & 26 \\
\hline 228 & Neu5Aca2-8Neu5Aca2-8Neu5Aca2-3Galb1-4Glcb-Sp0 & 10 & 8 & 81 \\
\hline 486 & $\begin{array}{l}\text { Galb1-4GIcNAcb1-6(Galb1-4GIcNAcb1-2)Mana1-6(Galb1-4GIcNAcb1-2Mana1-3)Manb1-4GIcNAcb1-4(Fuca1- } \\
\text { 6)GlcNAcb-Sp24 }\end{array}$ & 10 & 3 & 26 \\
\hline 144 & Galb1-3GalNAcb1-3Gala1-4Galb1-4GIcb-Sp0 & 10 & 6 & 64 \\
\hline 178 & GlcNAcb1-6(GlcNAcb1-3)GalNAca-Sp14 & 10 & 5 & 52 \\
\hline 111 & Gala1-3GalNAca-Sp8 & 10 & 9 & 93 \\
\hline 299 & (6P)Glcb-Sp10 & 10 & 2 & 22 \\
\hline 417 & Fuca1-2Galb1-4(Fuca1-3)GlcNAcb1-3GalNAca-Sp14 & 10 & 6 & 66 \\
\hline 242 & Neu5Aca2-3Galb1-4(Neu5Aca2-3Galb1-3)GlcNAcb-Sp8 & 10 & 13 & 135 \\
\hline 539 & Galb1-3GalNAcb1-3Gal-Sp21 & 10 & 9 & 95 \\
\hline 146 & Galb1-3GalNAcb1-4Galb1-4Glcb-Sp8 & 10 & 7 & 67 \\
\hline 109 & Gala1-3(Fuca1-2)Galb-Sp18 & 10 & 5 & 52 \\
\hline 275 & Neu5Aca2-6Galb-Sp8 & 10 & 6 & 62 \\
\hline 406 & Gala1-3Galb1-4GlcNAcb1-3GalNAca-Sp14 & 10 & 10 & 107 \\
\hline 14 & Manb-Sp8 & 10 & 3 & 34 \\
\hline 140 & Galb1-3GalNAca-Sp8 & 10 & 2 & 22 \\
\hline 143 & Galb1-3GalNAcb-Sp8 & 10 & 5 & 55 \\
\hline 324 & Neu5Gcb2-6Galb1-4GIcNAc-Sp8 & 9 & 5 & 58 \\
\hline 384 & Galb1-4(Fuca1-3)GIcNAcb1-6(Galb1-3GIcNAcb1-3)Galb1-4GIc-Sp21 & 9 & 8 & 81 \\
\hline 445 & $(6 S) G a l b 1-3(6 S) G l c N A c-S p 0$ & 9 & 5 & 55 \\
\hline 16 & GlcNAcb-Sp0 & 9 & 0 & 5 \\
\hline 221 & Fuca1-2(6S)Galb1-4GlcNAcb-Sp0 & 9 & 6 & 62 \\
\hline
\end{tabular}




\begin{tabular}{|c|c|c|c|c|}
\hline 463 & $\begin{array}{l}\text { Neu5Aca2-3Galb1-4GlcNAcb1-6(Neu5Aca2-3Galb1-4GIcNAcb1-2)Mana1-6(GlcNAcb1-4)(Neu5Aca2-3Galb1- } \\
\text { 4GIcNAcb1-4(Neu5Aca2-3Galb1-4GlcNAcb1-2)Mana1-3)Manb1-4GIcNAcb1-4GlcNAcb-Sp21 }\end{array}$ & 9 & 4 & 43 \\
\hline 31 & (3S)Galb1-3GlcNAcb-Sp8 & 9 & 8 & 84 \\
\hline 87 & GalNAca1-3(Fuca1-2)Galb1-4GIcNAcb-Sp8 & 9 & 7 & 71 \\
\hline 405 & Gala1-4Galb1-4GIcNAcb1-2Mana1-6(Gala1-4Galb1-4GIcNAcb1-2Mana1-3)Manb1-4GlcNAcb1-4GlcNAcb-Sp24 & 9 & 8 & 84 \\
\hline 51 & Mana1-6(Mana1-3)Manb1-4GlcNAcb1-4GlcNAcb-Sp13 & 9 & 5 & 54 \\
\hline 161 & Galb1-4GIcNAcb1-3GalNAc-Sp14 & 9 & 6 & 63 \\
\hline 166 & Galb1-4GlcNAcb1-3Galb1-4Glcb-Sp8 & 9 & 5 & 55 \\
\hline 126 & Galb1-2Galb-Sp8 & 9 & 6 & 65 \\
\hline 506 & Fuca1-2Galb1-3GIcNAcb1-6(Fuca1-2Galb1-3GIcNAcb1-3)GalNAca-Sp14 & 9 & 4 & 45 \\
\hline 227 & GalNAcb1-4(Neu5Aca2-8Neu5Aca2-8Neu5Aca2-3)Galb1-4Glcb-Sp0 & 9 & 9 & 95 \\
\hline 335 & GalNAcb1-3Gala1-4Galb1-4GIcNAcb1-3Galb1-4GIcb-Sp0 & 9 & 4 & 44 \\
\hline 63 & Fuca1-2Galb1-3GalNAca-Sp14 & 9 & 5 & 58 \\
\hline 136 & Neu5Aca2-6(Galb1-3)GalNAca-Sp8 & 9 & 1 & 9 \\
\hline 564 & GalNAcb1-4GIcNAcb1-3GalNAcb1-4GlcNAcb-Sp0 & 9 & 3 & 30 \\
\hline 165 & Galb1-4GlcNAcb1-3Galb1-4Glcb-Sp0 & 9 & 12 & 132 \\
\hline 205 & GlcAb1-6Galb-Sp8 & 9 & 5 & 57 \\
\hline 465 & $\begin{array}{l}\text { Neu5Aca2-6Galb1-4GIcNAcb1-4Mana1-6(GlcNAcb1-4)(Neu5Aca2-6Galb1-4GlcNAcb1-4(Neu5Aca2-6Galb1- } \\
\text { 4GIcNAcb1-2)Mana1-3)Manb1-4GIcNAcb1-4GIcNAcb-Sp21 }\end{array}$ & 9 & 6 & 69 \\
\hline 510 & Galb1-3GIcNAca1-3Galb1-4GIcNAcb-Sp8 & 9 & 3 & 36 \\
\hline 404 & Gala1-4Galb1-3GlcNAcb1-2Mana1-6(Gala1-4Galb1-3GlcNAcb1-2Mana1-3)Manb1-4GlcNAcb1-4GlcNAcb-Sp19 & 9 & 4 & 48 \\
\hline 186 & GlcNAcb1-3Galb1-4GIcb-Sp0 & 9 & 6 & 70 \\
\hline 308 & GlcAb1-3GIcNAcb-Sp8 & 9 & 3 & 31 \\
\hline 38 & (3S)Galb-Sp8 & 9 & 11 & 121 \\
\hline 208 & Mana1-2Mana1-2Mana1-3Mana-Sp9 & 9 & 4 & 44 \\
\hline 396 & $\begin{array}{l}\text { Gala1-3Galb1-3(Fuca1-4)GIcNAcb1-2Mana1-6(Gala1-3Galb1-3(Fuca1-4)GIcNAcb1-2Mana1-3)Manb1- } \\
\text { 4GlcNAcb1-4GIcNAc-Sp19 }\end{array}$ & 9 & 3 & 33 \\
\hline 53 & GlcNAcb1-2Mana1-6(GIcNAcb1-2Mana1-3)Manb1-4GlcNAcb1-4GIcNAcb-Sp13 & 9 & 6 & 69 \\
\hline 232 & GalNAcb1-4(Neu5Aca2-3)Galb1-4GlcNAcb-Sp0 & 9 & 5 & 61 \\
\hline 292 & Galb1-4(Fuca1-3)(6S)Glcb-Sp0 & 9 & 5 & 59 \\
\hline 74 & Fuca1-2Galb1-4GIcNAcb1-3Galb1-4GlcNAcb-Sp0 & 9 & 4 & 50 \\
\hline 392 & Galb1-3GIcNAcb1-3GalNAca-Sp14 & 9 & 5 & 53 \\
\hline 505 & (3S)GalNAcb1-4(Fuca1-3)GlcNAcb-Sp8 & 9 & 6 & 73 \\
\hline 182 & GlcNAcb1-3Galb-Sp8 & 9 & 9 & 100 \\
\hline 353 & GlcNAcb1-2Mana1-6(GlcNAcb1-2Mana1-3)Manb1-4GlcNAcb1-4(Fuca1-6)GlcNAcb-Sp22 & 9 & 5 & 51 \\
\hline 556 & Neu5Gca2-8Neu5Gca2-3Galb1-4GIcNAcb1-3Galb1-4GlcNAc-Sp0 & 9 & 3 & 37 \\
\hline 466 & $\begin{array}{l}\text { Neu5Aca2-6Galb1-4GIcNAcb1-6(Neu5Aca2-6Galb1-4GIcNAcb1-2)Mana1-6(GlcNAcb1-4)(Neu5Aca2-6Galb1- } \\
\text { 4GIcNAcb1-2Mana1-3)Manb1-4GIcNAcb1-4GIcNAcb-Sp21 }\end{array}$ & 9 & 11 & 127 \\
\hline 515 & GalNAcb1-4(6S)GlcNAc-Sp8 & 9 & 8 & 93 \\
\hline 218 & Manb1-4GlcNAcb-Sp0 & 9 & 17 & 197 \\
\hline
\end{tabular}




\begin{tabular}{|c|c|c|c|c|}
\hline 321 & GlcNAcb1-2Mana1-6(Neu5Aca2-6Galb1-4GIcNAcb1-2Mana1-3)Manb1-4GIcNAcb1-4GIcNAcb-Sp12 & 9 & 4 & 47 \\
\hline 303 & Galb1-4GIcNAcb1-6(Galb1-4GIcNAcb1-3)Galb1-4GIcNAc-Sp0 & 9 & 7 & 78 \\
\hline 113 & Gala1-3GalNAcb-Sp8 & 9 & 2 & 28 \\
\hline 92 & GalNAca1-3GalNAcb-Sp8 & 9 & 2 & 21 \\
\hline 70 & Fuca1-2Galb1-4(Fuca1-3)GıNAcb1-3Galb1-4(Fuca1-3)GIcNAcb-Sp0 & 9 & 5 & 54 \\
\hline 499 & Gala1-3(Fuca1-2)Galb1-4GIcNAcb1-6GalNAca-Sp14 & 8 & 4 & 45 \\
\hline 496 & Gala1-3Galb1-4GlcNAcb1-6GalNAca-Sp14 & 8 & 13 & 158 \\
\hline 356 & (6S)GlcNAcb1-3Galb1-4GlcNAcb-Sp0 & 8 & 3 & 40 \\
\hline 120 & Gala1-4(Fuca1-2)Galb1-4GlcNAcb-Sp8 & 8 & 8 & 99 \\
\hline 611 & Galb1-3GalNAcb1-4(Neu5Aca2-8Neu5Aca2-8Neu5Aca2-3)Galb1-4GIcb-Sp21 & 8 & 4 & 44 \\
\hline 452 & GalNAca1-3(Fuca1-2)Galb1-4GIcNAcb1-6(GalNAca1-3(Fuca1-2)Galb1-4GlcNAcb1-3)GalNAc-Sp14 & 8 & 7 & 80 \\
\hline 116 & Gala1-3Galb1-4GlcNAcb-Sp8 & 8 & 2 & 25 \\
\hline 160 & Galb1-4GlcNAcb1-3GalNAca-Sp8 & 8 & 6 & 71 \\
\hline 289 & Neu5Aca2-3Galb1-4GIcNAcb1-6(Galb1-3)GalNAca-Sp14 & 8 & 5 & 61 \\
\hline 369 & Galb1-4(Fuca1-3)GlcNAcb1-6(Fuca1-2Galb1-4GlcNAcb1-3)Galb1-4Glc-Sp21 & 8 & 2 & 24 \\
\hline 323 & Neu5Aca2-8Neu5Aca2-8Neu5Acb-Sp8 & 8 & 4 & 55 \\
\hline 594 & GlcNAcb1-3Galb1-4GIcNAcb1-3GalNAca-Sp14 & 8 & 6 & 68 \\
\hline 187 & GlcNAcb1-4-MDPLys & 8 & 1 & 17 \\
\hline 606 & $\begin{array}{l}\text { Neu5Aca2-6Galb1-4GIcNAcb1-3Galb1-4GIcNAcb1-6(Neu5Aca2-6Galb1-4GlcNAcb1-3Galb1-4GlcNAcb1- } \\
\text { 3)GalNAca-Sp14 }\end{array}$ & 8 & 12 & 151 \\
\hline 325 & Galb1-3GIcNAcb1-2Mana1-6(Galb1-3GlcNAcb1-2Mana1-3)Manb1-4GIcNAcb1-4GIcNAcb-Sp19 & 8 & 4 & 53 \\
\hline 129 & Galb1-3(Fuca1-4)GIcNAc-Sp0 & 8 & 10 & 124 \\
\hline 258 & Neu5Aca2-3Galb1-4(Fuca1-3)GlcNAcb1-3Galb1-4GlcNAcb-Sp8 & 8 & 8 & 98 \\
\hline 367 & Neu5Aca2-6GIcNAcb1-4GIcNAc-Sp21 & 8 & 4 & 51 \\
\hline 270 & Neu5Aca2-6Galb1-4GlcNAcb-Sp8 & 8 & 4 & 45 \\
\hline 83 & GalNAca1-3(Fuca1-2)Galb1-3GIcNAcb-Sp0 & 8 & 2 & 24 \\
\hline 58 & $\begin{array}{l}\text { Neu5Aca2-6Galb1-4GIcNAcb1-2Mana1-6(Neu5Aca2-6Galb1-4GlcNAcb1-2Mana1-3)Manb1-4GIcNAcb1- } \\
\text { 4GIcNAcb-Sp24 }\end{array}$ & 8 & 5 & 63 \\
\hline 386 & Galb1-4(Fuca1-3)GlcNAcb1-6(Fuca1-4(Fuca1-2Galb1-3)GlcNAcb1-3)Galb1-4Glc-Sp21 & 8 & 4 & 54 \\
\hline 329 & Neu5,9Ac2a2-3Galb1-4GIcNAcb-Sp0 & 8 & 4 & 50 \\
\hline 320 & Galb1-4GIcNAcb1-2Mana1-6(Neu5Aca2-6Galb1-4GIcNAcb1-2Mana1-3)Manb1-4GIcNAcb1-4GIcNAcb-Sp12 & 8 & 4 & 56 \\
\hline 207 & KDNa2-3Galb1-4GIcNAcb-Sp0 & 8 & 10 & 123 \\
\hline 391 & Fuca1-2Galb1-3GalNAca1-3(Fuca1-2)Galb1-4GIcNAcb-Sp0 & 8 & 16 & 206 \\
\hline 56 & $\begin{array}{l}\text { Neu5Aca2-6Galb1-4GIcNAcb1-2Mana1-6(Neu5Aca2-6Galb1-4GIcNAcb1-2Mana1-3)Manb1-4GIcNAcb1- } \\
\text { 4GIcNAcb-Sp13 }\end{array}$ & 8 & 8 & 99 \\
\hline 311 & GlcNAcb1-4GIcNAcb-Sp10 & 8 & 8 & 102 \\
\hline 425 & GalNAca1-3(Fuca1-2)Galb1-3GlcNAcb1-3GalNAc-Sp14 & 8 & 3 & 39 \\
\hline 27 & (3S)Galb1-4(6S)Glcb-Sp8 & 8 & 11 & 138 \\
\hline 44 & (6S)Galb1-4GlcNAcb-Sp8 & 8 & 12 & 151 \\
\hline 307 & GalNAcb1-3Galb-Sp8 & 8 & 5 & 72 \\
\hline
\end{tabular}




\begin{tabular}{|c|c|c|c|c|}
\hline 220 & (3S)Galb1-4(Fuca1-3)(6S)GlcNAcb-Sp8 & 8 & 2 & 22 \\
\hline 531 & GIcNAcb1-2 Mana1-6(GIcNAcb1-4)(GIcNAcb1-2Mana1-3)Manb1-4GIcNAcb1-4(Fuca1-6)GIcNAc-Sp21 & 8 & 5 & 66 \\
\hline 259 & Neu5Aca2-3Galb1-4GIcNAcb1-3Galb1-4GIcNAcb1-3Galb1-4GIcNAcb-Sp0 & 8 & 6 & 82 \\
\hline 112 & Gala1-3GalNAca-Sp16 & 8 & 5 & 68 \\
\hline 72 & Fuca1-2Galb1-4(Fuca1-3)GlcNAcb-Sp0 & 7 & 9 & 123 \\
\hline 238 & Neu5Aca2-3GalNAcb1-4GIcNAcb-Sp0 & 7 & 5 & 69 \\
\hline 66 & Fuca1-2Galb1-3GIcNAcb1-3Galb1-4GIcb-Sp8 & 7 & 4 & 53 \\
\hline 411 & Galb1-4GIcNAcb1-6(Neu5Aca2-6Galb1-3GIcNAcb1-3)Galb1-4Glc-Sp21 & 7 & 6 & 80 \\
\hline 162 & Galb1-4GIcNAcb1-3Galb1-4(Fuca1-3)GlcNAcb1-3Galb1-4(Fuca1-3)GlcNAcb-Sp0 & 7 & 5 & 66 \\
\hline 305 & Galb1-4GIcNAca1-6Galb1-4GIcNAcb-Sp0 & 7 & 7 & 92 \\
\hline 365 & Galb1-4GIcNAcb1-2Mana1-6(Mana1-3)Manb1-4GIcNAcb1-4GIcNAcb-Sp12 & 7 & 7 & 95 \\
\hline 432 & GlcNAcb1-2Mana1-6(GlcNAcb1-4)(GlcNAcb1-2Mana1-3)Manb1-4GlcNAcb1-4GlcNAc-Sp21 & 7 & 9 & 118 \\
\hline 123 & Gala1-4Galb1-4GIcb-Sp0 & 7 & 6 & 83 \\
\hline 383 & Galb1-3GlcNAcb1-3Galb1-4GIcNAcb1-6(Galb1-3GlcNAcb1-3)Galb1-4GIcb-Sp0 & 7 & 5 & 72 \\
\hline 192 & GlcNAcb1-4GIcNAcb1-4GIcNAcb-Sp8 & 7 & 4 & 57 \\
\hline 96 & GalNAcb1-3(Fuca1-2)Galb-Sp8 & 7 & 3 & 45 \\
\hline 8 & Rhaa-Sp8 & 7 & 4 & 49 \\
\hline 508 & $\begin{array}{l}\text { GlcNAcb1-6(GlcNAcb1-2)Mana1-6(GlcNAcb1-4)(GlcNAcb1-4(GlcNAcb1-2)Mana1-3)Manb1-4GlcNAcb1-4(Fuca1- } \\
\text { 6)GlcNAc-Sp21 }\end{array}$ & 7 & 4 & 63 \\
\hline 281 & Neu5Gca2-3Galb1-3(Fuca1-4)GIcNAcb-Sp0 & 7 & 4 & 60 \\
\hline 49 & Neu5,9Ac2a2-6Galb1-4GlcNAcb-Sp8 & 7 & 7 & 104 \\
\hline 211 & Mana1-6(Mana1-2Mana1-3)Mana1-6(Mana1-2Mana1-3)Manb1-4GIcNAcb1-4GIcNAcb-Sp12 & 7 & 3 & 37 \\
\hline 610 & GlcNAcb1-3Fuca-Sp21 & 7 & 7 & 103 \\
\hline 603 & Neu5Aca2-6Galb1-4GIcNAcb1-6(Galb1-3)GalNAca-Sp14 & 7 & 5 & 76 \\
\hline 198 & Glca1-6Glca1-6Glcb-Sp8 & 7 & 9 & 122 \\
\hline 412 & Galb1-3GalNAcb1-4(Neu5Aca2-8Neu5Aca2-3)Galb1-4Glcb-Sp0 & 7 & 4 & 53 \\
\hline 459 & $\begin{array}{l}\text { Galb1-4GIcNAcb1-6(Galb1-4GIcNAcb1-2)Mana1-6(Galb1-4GIcNAcb1-2Mana1-3)Manb1-4GIcNAcb1-4GIcNAcb- } \\
\text { Sp19 }\end{array}$ & 7 & 3 & 46 \\
\hline 290 & Galb1-3GlcNAcb1-3Galb1-3GIcNAcb-Sp0 & 7 & 2 & 29 \\
\hline 347 & Mana1-6(Neu5Aca2-6Galb1-4GIcNAcb1-2Mana1-3)Manb1-4GIcNAcb1-4GIcNAc-Sp12 & 7 & 7 & 95 \\
\hline 423 & Fuca1-2Galb1-3GlcNAcb1-3GalNAc-Sp14 & 7 & 3 & 38 \\
\hline 234 & GalNAcb1-4(Neu5Aca2-3)Galb1-4Glcb-Sp0 & 7 & 6 & 89 \\
\hline 255 & Neu5Aca2-3Galb1-4(Fuca1-3)GIcNAcb-Sp0 & 7 & 6 & 85 \\
\hline 555 & Neu5Gca2-8Neu5Aca2-3Galb1-4GIcNAc-Sp0 & 7 & 3 & 39 \\
\hline
\end{tabular}




\begin{tabular}{|c|c|c|c|c|}
\hline 224 & Neu5Aca2-3Galb1-3GalNAca-Sp8 & 7 & 6 & 88 \\
\hline 455 & $\begin{array}{l}\text { GalNAca1-3(Fuca1-2)Galb1-4GIcNAcb1-2Mana1-6(GalNAca1-3(Fuca1-2)Galb1-4GIcNAcb1-2Mana1-3)Manb1- } \\
\text { 4GIcNAcb1-4(Fuca1-6)GIcNAcb-Sp22 }\end{array}$ & 7 & 16 & 231 \\
\hline 344 & GlcNAca1-4Galb1-4GlcNAcb1-3Galb1-4GlcNAcb-Sp0 & 7 & 3 & 50 \\
\hline 520 & GalNAca1-3(Fuca1-2)Galb1-4GlcNAcb1-6GalNAc-Sp14 & 7 & 3 & 48 \\
\hline 598 & Neu5Aca2-6Galb1-4GIcNAcb1-3Galb1-4GIcNAcb1-3GalNAca-Sp14 & 7 & 5 & 75 \\
\hline 482 & Neu5Aca2-6Galb1-4 GlcNAcb1-6(Neu5Aca2-6Galb1-4GlcNAcb1-3)GalNAca-Sp14 & 7 & 3 & 47 \\
\hline 216 & Mana1-6(Mana1-3)Mana1-6(Mana1-2Mana1-3)Manb1-4GlcNAcb1-4GlcNAcb-Sp12 & 7 & 4 & 60 \\
\hline 86 & GaINAca1-3(Fuca1-2)Galb1-4GlcNAcb-Sp0 & 7 & 5 & 72 \\
\hline 195 & GlcNAcb1-6Galb1-4GlcNAcb-Sp8 & 7 & 4 & 69 \\
\hline 410 & Gala1-3(Fuca1-2)Galb1-4(Fuca1-3)Glcb-Sp21 & 6 & 3 & 45 \\
\hline 100 & GalNAcb1-4GIcNAcb-Sp8 & 6 & 5 & 78 \\
\hline 287 & Neu5Gca2-6Galb1-4GIcNAcb-Sp0 & 6 & 7 & 105 \\
\hline 244 & Neu5Aca2-6(Neu5Aca2-3Galb1-3)GalNAca-Sp8 & 6 & 2 & 35 \\
\hline 349 & Neu5Aca2-6Galb1-4GIcNAcb1-2Mana1-3Manb1-4GIcNAcb1-4GIcNAc-Sp12 & 6 & 2 & 39 \\
\hline 334 & Gala1-4Galb1-4GIcNAcb1-3Galb1-4GIcb-Sp0 & 6 & 2 & 33 \\
\hline 130 & Galb1-3(Fuca1-4)GIcNAc-Sp8 & 6 & 5 & 78 \\
\hline 210 & Mana1-2Mana1-3Mana-Sp9 & 6 & 4 & 67 \\
\hline 380 & GalNAcb1-4GIcNAcb1-2Mana1-6(GalNAcb1-4GIcNAcb1-2Mana1-3)Manb1-4GIcNAcb1-4GIcNAc-Sp12 & 6 & 2 & 37 \\
\hline 330 & Neu5,9Ac2a2-3Galb1-3GIcNAcb-Sp0 & 6 & 8 & 121 \\
\hline 263 & Fuca1-2Galb1-4(6S)Glcb-Sp0 & 6 & 6 & 97 \\
\hline 170 & Galb1-4GlcNAcb-Sp8 & 6 & 5 & 75 \\
\hline 388 & $\begin{array}{l}\text { Galb1-4GlcNAcb1-6(Galb1-4GlcNAcb1-2)Mana1-6(Galb1-4GlcNAcb1-4(Galb1-4GlcNAcb1-2)Mana1-3)Manb1- } \\
\text { 4GlcNAcb1-4GlcNAcb-Sp21 }\end{array}$ & 6 & 3 & 44 \\
\hline 563 & GalNAcb1-3GIcNAcb-Sp0 & 6 & 8 & 130 \\
\hline 60 & Fuca1-2Galb1-3GalNAcb1-3Gala1-4Galb1-4GIcb-Sp9 & 6 & 3 & 49 \\
\hline 525 & Galb1-3GIcNAcb1-2Mana-Sp0 & 6 & 5 & 81 \\
\hline 381 & Galb1-3GalNAca1-3(Fuca1-2)Galb1-4Glc-Sp0 & 6 & 9 & 154 \\
\hline 332 & Neu5Aca2-3Galb1-3(Fuca1-4)GIcNAcb1-3Galb1-3(Fuca1-4)GIcNAcb-Sp0 & 6 & 5 & 84 \\
\hline 163 & Galb1-4GlcNAcb1-3Galb1-4GlcNAcb1-3Galb1-4GlcNAcb-Sp0 & 6 & 5 & 82 \\
\hline 32 & (3S)Galb1-4(Fuca1-3)GlcNAc-Sp0 & 6 & 2 & 32 \\
\hline 127 & Galb1-3(Fuca1-4)GlcNAcb1-3Galb1-4(Fuca1-3)GlcNAcb-Sp0 & 6 & 6 & 96 \\
\hline 84 & GalNAca1-3(Fuca1-2)Galb1-4(Fuca1-3)GIcNAcb-Sp0 & 6 & 4 & 63 \\
\hline 518 & Galb1-4(6P)GlcNAcb-Sp0 & 6 & 8 & 139 \\
\hline 138 & Neu5Acb2-6(Galb1-3)GalNAca-Sp8 & 6 & 6 & 96 \\
\hline 283 & Neu5Gca2-3Galb1-4(Fuca1-3)GlcNAcb-Sp0 & 6 & 4 & 71 \\
\hline 254 & Neu5Aca2-3Galb1-4(Fuca1-3)GlcNAcb1-3Galb1-4(Fuca1-3)GlcNAcb1-3Galb1-4(Fuca1-3)GIcNAcb-Sp0 & 6 & 5 & 95 \\
\hline 295 & Neu5Aca2-3Galb1-3GlcNAcb1-3Galb1-3GlcNAcb-Sp0 & 6 & 5 & 81 \\
\hline 240 & Neu5Aca2-3Galb1-3(Fuca1-4)GIcNAcb-Sp8 & 6 & 5 & 91 \\
\hline
\end{tabular}




\begin{tabular}{|c|c|c|c|c|}
\hline 393 & GalNAcb1-4(Neu5Aca2-3)Galb1-4GIcNAcb1-3GalNAca-Sp14 & 6 & 6 & 106 \\
\hline 407 & Galb1-3GIcNAcb1-6Galb1-4GIcNAcb-Sp0 & 6 & 6 & 97 \\
\hline 415 & GalNAca1-3(Fuca1-2)Galb1-4GIcNAcb1-3GalNAca-Sp14 & 6 & 2 & 39 \\
\hline 493 & (3S)Galb1-3(Fuca1-4)GIcNAcb-Sp0 & 6 & 5 & 86 \\
\hline 191 & GlcNAcb1-4GlcNAcb1-4GlcNAcb1-4GlcNAcb1-4GlcNAcb1-Sp8 & 5 & 7 & 122 \\
\hline 491 & Galb1-3(Fuca1-4)GlcNAcb1-6GalNAca-Sp14 & 5 & 8 & 141 \\
\hline 581 & $\begin{array}{l}\text { GlcNAcb1-3Galb1-4GlcNAcb1-3Galb1-4GlcNAcb1-3Galb1-4GlcNAcb1-3Galb1-4GlcNAcb1-2Mana1-6(GlcNAcb1- } \\
\text { 3Galb1-4GlcNAcb1-3Galb1-4GlcNAcb1-3Galb1-4GlcNAcb1-3Galb1-4GIcNAcb1-2Mana1-3)Manb1-4GlcNAcb1- } \\
\text { 4(Fuca1-6)GlcNAcb-Sp19 }\end{array}$ & 5 & 4 & 71 \\
\hline 137 & Neu5Aca2-6(Galb1-3)GalNAca-Sp14 & 5 & 5 & 92 \\
\hline 512 & (6S)(4S)GalNAcb1-4GlcNAc-Sp8 & 5 & 1 & 26 \\
\hline 595 & GIcNAcb1-3Galb1-4GIcNAcb1-6(Galb1-3)GalNAca-Sp14 & 5 & 4 & 76 \\
\hline 209 & Mana1-2Mana1-6(Mana1-2Mana1-3)Mana-Sp9 & 5 & 6 & 108 \\
\hline 193 & GlcNAcb1-6GalNAca-Sp8 & 5 & 4 & 67 \\
\hline 131 & Fuca1-4(Galb1-3)GlcNAcb-Sp8 & 5 & 3 & 54 \\
\hline 317 & Mana1-2Mana1-6(Mana1-2Mana1-3)Mana1-6(Mana1-2Mana1-2Mana1-3)Mana-Sp9 & 5 & 4 & 78 \\
\hline 36 & (3S)Galb1-4GlcNAcb-Sp0 & 5 & 6 & 122 \\
\hline 177 & GlcNAcb1-6(GlcNAcb1-3)GalNAca-Sp8 & 5 & 4 & 72 \\
\hline 313 & MurNAcb1-4GIcNAcb-Sp10 & 5 & 15 & 287 \\
\hline 297 & $4 S(3 S) G a l b 1-4 G I c N A c b-S p 0$ & 5 & 6 & 113 \\
\hline 171 & Galb1-4GlcNAcb-Sp23 & 5 & 3 & 59 \\
\hline 54 & Galb1-4GIcNAcb1-2Mana1-6(Galb1-4GIcNAcb1-2Mana1-3)Manb1-4GIcNAcb1-4GIcNAcb-Sp12 & 5 & 5 & 94 \\
\hline 429 & $\begin{array}{l}\text { Galb1-3GlcNAcb1-6(Galb1-3GlcNAcb1-2)Mana1-6(Galb1-3GlcNAcb1-2Mana1-3)Manb1-4GlcNAcb1-4GlcNAcb- } \\
\text { Sp19 }\end{array}$ & 5 & 5 & 97 \\
\hline 280 & Neu5Acb2-6Galb1-4GlcNAcb-Sp8 & 5 & 2 & 41 \\
\hline 472 & Neu5Aca2-3Galb1-4GIcNAcb1-6(Neu5Aca2-3Galb1-4GIcNAcb1-3)GalNAca-Sp14 & 5 & 7 & 146 \\
\hline 164 & Galb1-4GIcNAcb1-3Galb1-4GIcNAcb-Sp0 & 5 & 5 & 102 \\
\hline 202 & GlcAa-Sp8 & 5 & 3 & 52 \\
\hline 591 & Galb1-4GIcNAcb1-3Galb1-4GIcNAcb1-6(Galb1-3)GalNAca-Sp14 & 5 & 5 & 101 \\
\hline 351 & Galb1-4GIcNAcb1-2Mana1-6Manb1-4GlcNAcb1-4GIcNAc-Sp12 & 5 & 4 & 81 \\
\hline 247 & Neu5Aca2-3Galb1-3GalNAcb1-3Gala1-4Galb1-4Glcb-Sp0 & 5 & 5 & 99 \\
\hline 368 & Neu5Aca2-6GIcNAcb1-4GIcNAcb1-4GIcNAc-Sp21 & 5 & 4 & 80 \\
\hline 342 & GIcNAca1-4Galb1-4GIcNAcb1-3Galb1-4Glcb-Sp0 & 5 & 7 & 141 \\
\hline 266 & Neu5Aca2-6GalNAca-Sp8 & 5 & 5 & 108 \\
\hline 128 & Galb1-3GIcNAcb1-3Galb1-4(Fuca1-3)GIcNAcb-Sp0 & 5 & 3 & 68 \\
\hline 419 & GalNAca1-3(Fuca1-2)Galb1-4(Fuca1-3)GlcNAcb1-3GalNAc-Sp14 & 5 & 2 & 35 \\
\hline 526 & Gala1-3(Fuca1-2)Galb1-3GIcNAcb1-6GalNAc-Sp14 & 5 & 6 & 130 \\
\hline
\end{tabular}




\begin{tabular}{|c|c|c|c|c|}
\hline 358 & KDNa2-6Galb1-4GIcNAc-Sp0 & 5 & 3 & 62 \\
\hline 273 & Neu5Aca2-6Galb1-4GIcb-Sp0 & 5 & 2 & 43 \\
\hline 190 & GlcNAcb1-4GlcNAcb1-4GIcNAcb1-4GlcNAcb1-4GlcNAcb1-4GlcNAcb1-Sp8 & 5 & 9 & 194 \\
\hline 59 & Fuca1-2Galb1-3GalNAcb1-3Gala-Sp9 & 5 & 3 & 62 \\
\hline 562 & GlcNAcb1-3Galb1-4GlcNAcb1-6(GlcNAcb1-3Galb1-3)GalNAca-Sp14 & 5 & 4 & 93 \\
\hline 294 & Galb1-4GIcNAcb1-3Galb1-3GIcNAcb-Sp0 & 5 & 3 & 71 \\
\hline 458 & $\begin{array}{l}\text { GalNAca1-3(Fuca1-2)Galb1-3GIcNAcb1-2Mana1-6(GalNAca1-3(Fuca1-2)Galb1-3GlcNAcb1-2Mana1-3)Manb1- } \\
\text { 4GIcNAcb1-4(Fuca1-6)GIcNAcb-Sp22 }\end{array}$ & 4 & 4 & 90 \\
\hline 422 & GlcNAcb1-2(GIcNAcb1-6)Mana1-6(GIcNAcb1-2Mana1-3)Manb1-4GIcNAcb1-4GIcNAcb-Sp19 & 4 & 8 & 189 \\
\hline 485 & Mana1-6(Mana1-3)Manb1-4GlcNAcb1-4(Fuca1-6)GlcNAcb-Sp19 & 4 & 4 & 91 \\
\hline 250 & Neu5Aca2-3Galb1-3GIcNAcb-Sp0 & 4 & 6 & 130 \\
\hline 284 & Neu5Gca2-3Galb1-4GlcNAcb-Sp0 & 4 & 3 & 65 \\
\hline 214 & Mana1-6(Mana1-3)Mana-Sp9 & 4 & 4 & 92 \\
\hline 504 & GalNAcb1-4(Fuca1-3)(6S)GIcNAcb-Sp8 & 4 & 6 & 134 \\
\hline 46 & Neu5Aca2-3(6S)Galb1-4GIcNAcb-Sp8 & 4 & 6 & 146 \\
\hline 185 & GIcNAcb1-3Galb1-4GIcNAcb1-3Galb1-4GIcNAcb-Sp0 & 4 & 9 & 209 \\
\hline 345 & GlcNAca1-4Galb1-3GalNAc-Sp14 & 4 & 9 & 209 \\
\hline 189 & GlcNAcb1-4Galb1-4GIcNAcb-Sp8 & 4 & 2 & 48 \\
\hline 249 & Fuca1-2(6S)Galb1-4GIcb-Sp0 & 4 & 2 & 46 \\
\hline 2 & Glca-Sp8 & 4 & 5 & 134 \\
\hline 489 & Galb1-3GlcNAcb1-6GalNAca-Sp14 & 4 & 2 & 42 \\
\hline 95 & GalNAcb1-3GalNAca-Sp8 & 4 & 7 & 182 \\
\hline 341 & GlcNAca1-4Galb1-3GlcNAcb-Sp0 & 4 & 8 & 215 \\
\hline 15 & GalNAcb-Sp8 & 4 & 11 & 289 \\
\hline 62 & Fuca1-2Galb1-3GalNAca-Sp8 & 4 & 3 & 90 \\
\hline 261 & Neu5Aca2-3Galb1-4GIcNAcb-Sp8 & 4 & 5 & 139 \\
\hline 536 & GlcNAcb1-3Galb1-4GIcNAcb1-6(GlcNAcb1-3)Galb1-4GlcNAc-Sp0 & 4 & 15 & 388 \\
\hline 481 & Neu5Aca2-6Galb1-4GIcNAcb1-6GalNAca-Sp14 & 4 & 3 & 82 \\
\hline 269 & Neu5Aca2-6Galb1-4GIcNAcb-Sp0 & 4 & 5 & 137 \\
\hline 282 & Neu5Gca2-3Galb1-3GlcNAcb-Sp0 & 4 & 13 & 347 \\
\hline 118 & Gala1-3Galb1-4Glc-Sp10 & 4 & 5 & 144 \\
\hline
\end{tabular}




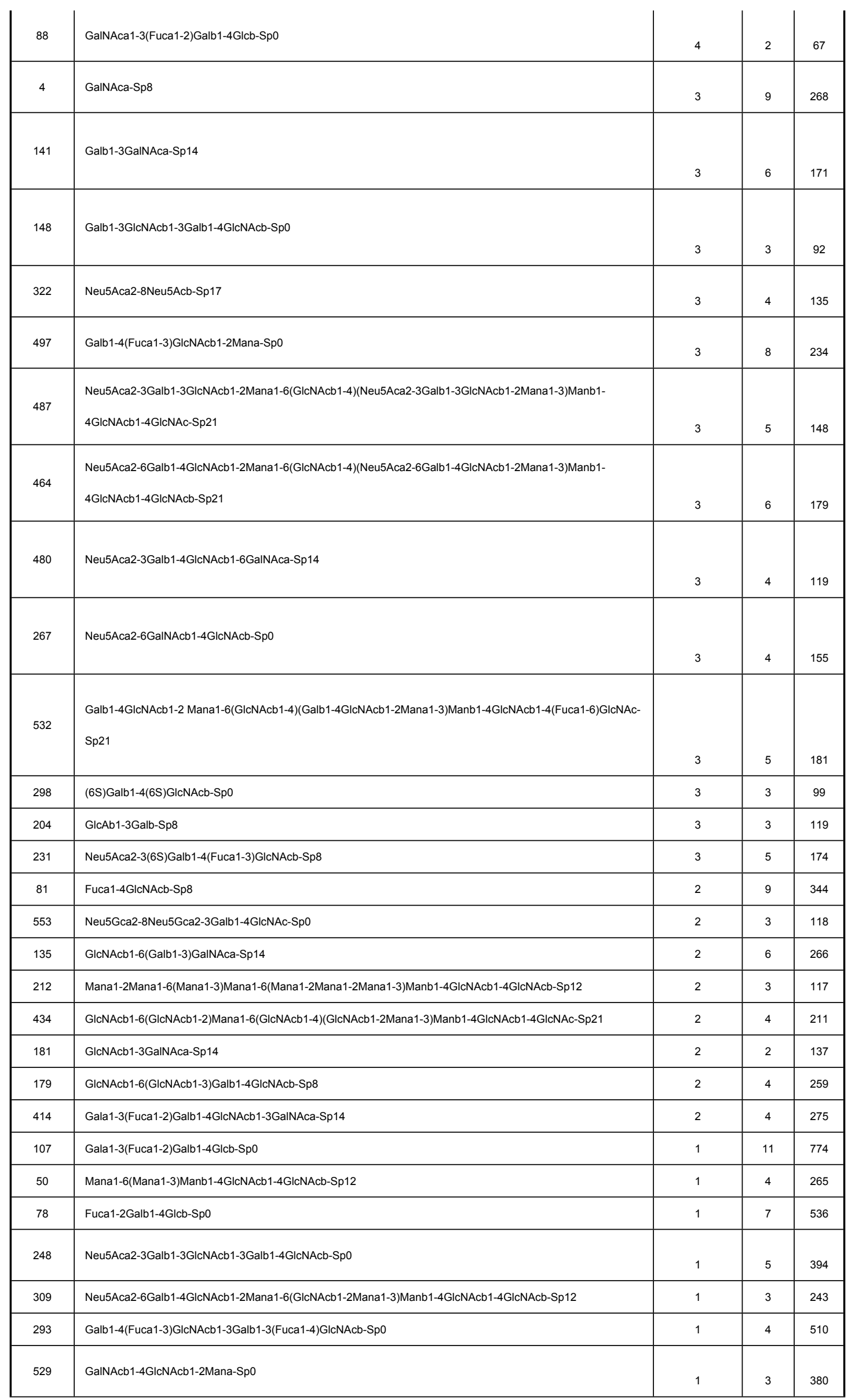




\begin{tabular}{|c|l|c|c|c|}
\hline 5 & GalNAca-Sp15 & 1 & 4 & 426 \\
\hline 375 & $\begin{array}{l}\text { Gala1-3(Fuca1-2)Galb1-3GIcNAcb1-2Mana1-6(Gala1-3(Fuca1-2)Galb1-3GIcNAcb1-2Mana1-3)Manb1- } \\
\text { 4GIcNAcb1-4GIcNAcb-Sp20 }\end{array}$ & 0 & 6 & 3265 \\
\hline 558 & Neu5Aca2-8Neu5Aca2-3Galb1-4GIcNAc-Sp0 & 0 & 1 & -137 \\
\hline 75 & Fuca1-2Galb1-4GIcNAcb1-3Galb1-4GIcNAcb1-3Galb1-4GIcNAcb-Sp0 & -2 & 6 & -394 \\
\hline
\end{tabular}

Supporting data 4. rAAL-mut63H(R63H)_200ug_15885_V5.1_DATA

\begin{tabular}{|c|c|c|c|c|}
\hline $\begin{array}{l}\text { Chart } \\
\text { Number }\end{array}$ & rAAL_mut63H (200ug/ml) Slide\#:15885 Cfg2648 V5.1 10/22/2012 Alexa488 CMZ & Average RFU & StDev & $\% \mathrm{CV}$ \\
\hline 570 & (3S)GlcAb1-3Galb1-4GIcNAcb1-2Mana-Sp0 & 248 & 12 & 5 \\
\hline 437 & $\begin{array}{l}\text { Galb1-4GlcNAcb1-6(Galb1-4GlcNAcb1-2)Mana1-6(GlcNAcb1-4)(Galb1-4GlcNAcb1-2Mana1-3)Manb1- } \\
\text { 4GlcNAcb1-4GIcNAc-Sp21 }\end{array}$ & 179 & 194 & 109 \\
\hline 569 & (3S)GlcAb1-3Galb1-4GlcNAcb1-3Galb1-4Glc-Sp0 & 166 & 22 & 13 \\
\hline 17 & GlcNAcb-Sp8 & 89 & 79 & 89 \\
\hline 461 & $\begin{array}{l}\text { Neu5Aca2-3Galb1-4GlcNAcb1-6(Neu5Aca2-3Galb1-4GlcNAcb1-2)Mana1-6(GlcNAcb1-4)(Neu5Aca2-3Galb1- } \\
\text { 4GlcNAcb1-2Mana1-3)Manb1-4GlcNAcb1-4GlcNAcb-Sp21 }\end{array}$ & 76 & 97 & 128 \\
\hline 185 & GlcNAcb1-3Galb1-4Glcb-Sp0 & 71 & 89 & 125 \\
\hline 64 & Fuca1-2Galb1-3GalNAcb1-4(Neu5Aca2-3)Galb1-4Glcb-Sp9 & 66 & 89 & 134 \\
\hline 284 & Neu5Gca2-3Galb1-4GIcb-Sp0 & 65 & 52 & 79 \\
\hline 577 & $\begin{array}{l}\text { Galb1-4GIcNAcb1-3Galb1-4GlcNAcb1-3Galb1-4GIcNAcb1-2Mana1-6(Galb1-4GlcNAcb1-3Galb1-4GIcNAcb1- } \\
\text { 3Galb1-4GIcNAcb1-2Mana1-3)Manb1-4GlcNAcb1-4(Fuca1-6)GIcNAcb-Sp24 }\end{array}$ & 62 & 10 & 16 \\
\hline 509 & Galb1-3GIcNAca1-3Galb1-4GIcNAcb-Sp8 & 53 & 29 & 54 \\
\hline 541 & $\begin{array}{l}\text { Galb1-4GlcNAcb1-3Galb1-4GIcNAcb1-2Mana1-6(Galb1-4GlcNAcb1-3Galb1-4GlcNAcb1-2Mana1-3)Manb1- } \\
\text { 4GIcNAcb1-4GlcNAcb-Sp12 }\end{array}$ & 52 & 5 & 9 \\
\hline 404 & Gala1-4Galb1-4GIcNAcb1-2Mana1-6(Gala1-4Galb1-4GIcNAcb1-2Mana1-3)Manb1-4GIcNAcb1-4GlcNAcb-Sp24 & 51 & 11 & 21 \\
\hline 489 & Gala1-3Galb1-3GlcNAcb1-6GalNAca-Sp14 & 51 & 18 & 36 \\
\hline 256 & Neu5Aca2-3Galb1-4(Fuca1-3)GIcNAcb1-3Galb-Sp8 & 51 & 21 & 41 \\
\hline 585 & $\begin{array}{l}\text { GlcNAcb1-3Galb1-4GlcNAcb1-3Galb1-4GlcNAcb1-3Galb1-4GlcNAcb1-6(GlcNAcb1-3Galb1-4GlcNAcb1- } \\
\text { 3Galb1-4GlcNAcb1-3Galb1-4GlcNAb1-2)Mana1-6(GlcNAcb1-3Galb1-4GlcNAcb1-3Galb1-4GIcNAcb1-3Galb1- } \\
\text { 4GlcNAcb1-2Mana1-3)Manb1-4GlcNAcb1-4(Fuca1-6)GlcNAcb-Sp24 }\end{array}$ & 50 & 20 & 39 \\
\hline 583 & $\begin{array}{l}\text { GlcNAcb1-3Galb1-4GIcNAcb1-3Galb1-4GIcNAcb1-6(GlcNAcb1-3Galb1-4GIcNAcb1-3Galb1-4GIcNAb1- } \\
\text { 2)Mana1-6(GIcNAcb1-3Galb1-4GIcNAcb1-3Galb1-4GIcNAcb1-2Mana1-3)Manb1-4GIcNAcb1-4(Fuca1- } \\
\text { 6)GlcNAcb-Sp24 }\end{array}$ & 50 & 14 & 27 \\
\hline 578 & $\begin{array}{l}\text { GlcNAcb1-3Galb1-4GlcNAcb1-3Galb1-4GlcNAcb1-3Galb1-4GIcNAcb1-2Mana1-6(GlcNAcb1-3Galb1- } \\
\text { 4GlcNAcb1-3Galb1-4GlcNAcb1-3Galb1-4GlcNAcb1-2Mana1-3)Manb1-4GlcNAcb1-4(Fuca1-6)GlcNAcb-Sp24 }\end{array}$ & 49 & 4 & 7 \\
\hline 572 & $\begin{array}{l}\text { Galb1-3GIcNAcb1-3Galb1-4GlcNAcb1-6(Galb1-3GlcNAcb1-3Galb1-4GlcNAb1-2)Mana1-6(Galb1-3GlcNAcb1- } \\
\text { 3Galb1-4GlcNAcb1-2Mana1-3)Manb1-4GlcNAcb1-4(Fuca1-6)GlcNAcb-Sp24 }\end{array}$ & 49 & 19 & 39 \\
\hline 587 & $\begin{array}{l}\text { GlcNAcb1-3Galb1-4GIcNAcb1-3Galb1-4GlcNAcb1-3Galb1-4GIcNAcb1-3Galb1-4GlcNAcb1-6(GlcNAcb1- } \\
\text { 3Galb1-4GIcNAcb1-3Galb1-4GlcNAcb1-3Galb1-4GIcNAcb1-3Galb1-4GIcNAb1-2)Mana1-6(GlcNAcb1-3Galb1- } \\
\text { 4GlcNAcb1-3Galb1-4GIcNAcb1-3Galb1-4GIcNAcb1-3Galb1-4GIcNAcb1-2Mana1-3)Manb1-4GlcNAcb1- } \\
\text { 4(Fuca1-6)GlcNAcb-Sp24 }\end{array}$ & 48 & 3 & 6 \\
\hline
\end{tabular}




\begin{tabular}{|c|c|c|c|c|}
\hline 162 & Galb1-4GlcNAcb1-3Galb1-4GlcNAcb1-3Galb1-4GlcNAcb-Sp0 & 46 & 6 & 14 \\
\hline 385 & Galb1-4(Fuca1-3)GIcNAcb1-6(Fuca1-4(Fuca1-2Galb1-3)GIcNAcb1-3)Galb1-4GIc-Sp21 & 46 & 10 & 21 \\
\hline 85 & GalNAca1-3(Fuca1-2)Galb1-4GIcNAcb-Sp0 & 44 & 37 & 84 \\
\hline 482 & $\begin{array}{l}\text { Neu5Aca2-6Galb1-4GlcNAcb1-2Mana1-6(Neu5Aca2-6Galb1-4GlcNAcb1-2Mana1-3)Manb1-4GlcNAcb1- } \\
\text { 4(Fuca1-6)GlcNAcb-Sp24 }\end{array}$ & 43 & 9 & 22 \\
\hline 484 & Mana1-6(Mana1-3)Manb1-4GlcNAcb1-4(Fuca1-6)GlcNAcb-Sp19 & 43 & 9 & 21 \\
\hline 227 & Neu5Aca2-8Neu5Aca2-8Neu5Aca2-3Galb1-4Glcb-Sp0 & 42 & 18 & 42 \\
\hline 584 & $\begin{array}{l}\text { Galb1-4GIcNAcb1-3Galb1-4GlcNAcb1-3Galb1-4GlcNAcb1-6(Galb1-4GlcNAcb1-3Galb1-4GlcNAcb1-3Galb1- } \\
\text { 4GlcNAb1-2)Mana1-6(Galb1-4GlcNAcb1-3Galb1-4GIcNAcb1-3Galb1-4GlcNAcb1-2Mana1-3)Manb1- } \\
\text { 4GlcNAcb1-4(Fuca1-6)GlcNAcb-Sp24 }\end{array}$ & 42 & 10 & 23 \\
\hline 586 & $\begin{array}{l}\text { Galb1-4GlcNAcb1-3Galb1-4GlcNAcb1-3Galb1-4GIcNAcb1-3Galb1-4GlcNAcb1-6(Galb1-4GlcNAcb1-3Galb1- } \\
\text { 4GIcNAcb1-3Galb1-4GIcNAcb1-3Galb1-4GlcNAb1-2)Mana1-6(Galb1-4GIcNAcb1-3Galb1-4GIcNAcb1-3Galb1- } \\
\text { 4GlcNAcb1-3Galb1-4GIcNAcb1-2Mana1-3)Manb1-4GlcNAcb1-4(Fuca1-6)GlcNAcb-Sp24 }\end{array}$ & 41 & 11 & 27 \\
\hline 24 & (3S)Galb1-4(Fuca1-3)(6S)Glc-Sp0 & 40 & 10 & 24 \\
\hline 533 & Fuca1-4(Galb1-3)GlcNAcb1-2 Mana-Sp0 & 40 & 15 & 37 \\
\hline 538 & Galb1-3GalNAcb1-3Gal-Sp21 & 40 & 37 & 91 \\
\hline 513 & (3S)GalNAcb1-4(3S)GIcNAc-Sp8 & 40 & 15 & 37 \\
\hline 595 & GlcNAcb1-3Galb1-4GIcNAcb1-6(GIcNAcb1-3Galb1-4GlcNAcb1-3)GalNAca-Sp14 & 40 & 14 & 34 \\
\hline 165 & Galb1-4GIcNAcb1-3Galb1-4Glcb-Sp8 & 40 & 17 & 42 \\
\hline 138 & Neu5Aca2-6(Galb1-3)GlcNAcb1-4Galb1-4Glcb-Sp10 & 40 & 16 & 41 \\
\hline 112 & Gala1-3GalNAcb-Sp8 & 39 & 22 & 57 \\
\hline 135 & Neu5Aca2-6(Galb1-3)GalNAca-Sp8 & 38 & 13 & 34 \\
\hline 166 & Galb1-4GlcNAcb1-6(Galb1-3)GalNAca-Sp8 & 38 & 54 & 142 \\
\hline 42 & (6S)Galb1-4Glcb-Sp0 & 38 & 25 & 65 \\
\hline 231 & GalNAcb1-4(Neu5Aca2-3)Galb1-4GlcNAcb-Sp0 & 38 & 5 & 14 \\
\hline 607 & $\begin{array}{l}\text { Neu5Aca2-3Galb1-4GIcNAcb1-3Galb1-4GIcNAcb1-3Galb1-4GIcNAcb1-2Mana1-6(Neu5Aca2-3Galb1- } \\
\text { 4GIcNAcb1-3Galb1-4GIcNAcb1-3Galb1-4GIcNAcb1-2Mana1-3)Manb1-4GIcNAcb1-4GIcNAcb-Sp12 }\end{array}$ & 37 & 7 & 20 \\
\hline 362 & $\begin{array}{l}\text { Fuca1-2Galb1-4(Fuca1-3)GIcNAcb1-2Mana1-6(Fuca1-2Galb1-4(Fuca1-3)GlcNAcb1-2Mana1-3)Manb1- } \\
\text { 4GIcNAcb1-4GIcNAb-Sp20 }\end{array}$ & 37 & 22 & 58 \\
\hline 98 & GalNAcb1-4GlcNAcb-Sp0 & 37 & 8 & 22 \\
\hline 516 & (4S)GalNAcb-Sp10 & 36 & 16 & 44 \\
\hline 96 & GalNAcb1-3Gala1-4Galb1-4GIcNAcb-Sp0 & 36 & 9 & 24 \\
\hline 18 & GlcN(Gc)b-Sp8 & 36 & 4 & 12 \\
\hline 408 & GalNAcb1-3Gala1-6Galb1-4Glcb-Sp8 & 35 & 11 & 32 \\
\hline 63 & Fuca1-2Galb1-3GalNAcb1-4(Neu5Aca2-3)Galb1-4Glcb-Sp0 & 35 & 17 & 49 \\
\hline 505 & Fuca1-2Galb1-3GIcNAcb1-6(Fuca1-2Galb1-3GIcNAcb1-3)GalNAca-Sp14 & 34 & 10 & 30 \\
\hline 354 & Galb1-3GlcNAcb1-2Mana1-6(Galb1-3GlcNAcb1-2Mana1-3)Manb1-4GIcNAcb1-4(Fuca1-6)GIcNAcb-Sp22 & 34 & 16 & 47 \\
\hline 14 & Manb-Sp8 & 34 & 10 & 29 \\
\hline 361 & $\begin{array}{l}\text { Fuca1-2Galb1-4GIcNAcb1-2Mana1-6(Fuca1-2Galb1-4GIcNAcb1-2Mana1-3)Manb1-4GIcNAcb1-4GIcNAcb- } \\
\text { Sp20 }\end{array}$ & 34 & 11 & 33 \\
\hline 493 & Galb1-4(Fuca1-3)GlcNAcb1-6(Neu5Aca2-6(Neu5Aca2-3Galb1-3)GlcNAcb1-3)Galb1-4Glc-Sp21 & 34 & 9 & 25 \\
\hline
\end{tabular}




\begin{tabular}{|c|c|c|c|c|}
\hline 580 & $\begin{array}{l}\text { GlcNAcb1-3Galb1-4GlcNAcb1-3Galb1-4GIcNAcb1-3Galb1-4GIcNAcb1-3Galb1-4GlcNAcb1-2Mana1- } \\
\text { 6(GlcNAcb1-3Galb1-4GlcNAcb1-3Galb1-4GIcNAcb1-3Galb1-4GlcNAcb1-3Galb1-4GlcNAcb1-2Mana1- } \\
\text { 3)Manb1-4GlcNAcb1-4(Fuca1-6)GlcNAcb-Sp19 }\end{array}$ & 34 & 12 & 35 \\
\hline 452 & Neu5Aca2-8Neu5Aca2-3Galb1-3GalNAcb1-4(Neu5Aca2-8Neu5Aca2-3)Galb1-4Glcb-Sp0 & 34 & 14 & 41 \\
\hline 468 & Gala1-3(Fuca1-2)Galb1-3GalNAcb-Sp8 & 34 & 20 & 59 \\
\hline 515 & (3S)GalNAcb1-4GIcNAc-Sp8 & 33 & 8 & 24 \\
\hline 605 & $\begin{array}{l}\text { Neu5Aca2-6Galb1-4GIcNAcb1-3Galb1-4GIcNAcb1-6(Neu5Aca2-6Galb1-4GlcNAcb1-3Galb1-4GlcNAcb1- } \\
\text { 3)GalNAca-Sp14 }\end{array}$ & 33 & 5 & 16 \\
\hline 576 & $\begin{array}{l}\text { GlcNAcb1-3Galb1-4GlcNAcb1-3Galb1-4GlcNAcb1-2Mana1-6(GlcNAcb1-3Galb1-4GlcNAcb1-3Galb1- } \\
\text { 4GIcNAcb1-2Mana1-3)Manb1-4GlcNAcb1-4(Fuca1-6)GlcNAcb-Sp24 }\end{array}$ & 33 & 15 & 46 \\
\hline 573 & Neu5Aca2-8Neu5Aca2-3Galb1-3GalNAcb1-4(Neu5Aca2-3)Galb1-4Glc-Sp21 & 33 & 12 & 37 \\
\hline 23 & $6 \mathrm{~S}(3 \mathrm{~S})$ Galb1-4GlcNAcb-Sp0 & 33 & 10 & 32 \\
\hline 204 & GlcAb1-6Galb-Sp8 & 32 & 31 & 96 \\
\hline 288 & Neu5Aca2-3Galb1-4GIcNAcb1-6(Galb1-3)GalNAca-Sp14 & 32 & 19 & 59 \\
\hline 208 & Mana1-2Mana1-6(Mana1-2Mana1-3)Mana-Sp9 & 32 & 6 & 20 \\
\hline 514 & GalNAcb1-4(6S)GlcNAc-Sp8 & 32 & 12 & 38 \\
\hline 485 & $\begin{array}{l}\text { Galb1-4GIcNAcb1-6(Galb1-4GIcNAcb1-2)Mana1-6(Galb1-4GlcNAcb1-2Mana1-3)Manb1-4GIcNAcb1-4(Fuca1- } \\
\text { 6)GIcNAcb-Sp24 }\end{array}$ & 32 & 15 & 48 \\
\hline 579 & $\begin{array}{l}\text { Galb1-4GIcNAcb1-3Galb1-4GlcNAcb1-3Galb1-4GlcNAcb1-3Galb1-4GlcNAcb1-2Mana1-6(Galb1-4GlcNAcb1- } \\
\text { 3Galb1-4GlcNAcb1-3Galb1-4GlcNAcb1-3Galb1-4GlcNAcb1-2Mana1-3)Manb1-4GlcNAcb1-4(Fuca1- } \\
\text { 6)GlcNAcb-Sp24 }\end{array}$ & 32 & 7 & 21 \\
\hline 568 & $\begin{array}{l}\text { Galb1-4GIcNAcb1-3Galb1-4GlcNAcb1-3Galb1-4GlcNAcb1-3Galb1-4GlcNAcb1-3Galb1-4GlcNAcb1-3Galb1- } \\
\text { 4GlcNAcb1-2Mana1-6(Galb1-4GlcNAcb1-3Galb1-4GlcNAcb1-3Galb1-4GlcNAcb1-3Galb1-4GlcNAcb1-3Galb1- } \\
\text { 4GlcNAcb1-3Galb1-4GlcNAcb1-2Mana1-3)Manb1-4GlcNAcb1-4GlcNAcb-Sp25 }\end{array}$ & 32 & 5 & 17 \\
\hline 161 & Galb1-4GlcNAcb1-3Galb1-4(Fuca1-3)GlcNAcb1-3Galb1-4(Fuca1-3)GlcNAcb-Sp0 & 32 & 19 & 59 \\
\hline 582 & $\begin{array}{l}\text { Galb1-4GIcNAcb1-3Galb1-4GlcNAcb1-6(Galb1-4GlcNAcb1-3Galb1-4GlcNAb1-2)Mana1-6(Galb1-4GlcNAcb1- } \\
\text { 3Galb1-4GlcNAcb1-2Mana1-3)Manb1-4GlcNAcb1-4(Fuca1-6)GlcNAcb-Sp24 }\end{array}$ & 32 & 15 & 47 \\
\hline 413 & Gala1-3(Fuca1-2)Galb1-4GIcNAcb1-3GalNAca-Sp14 & 31 & 19 & 62 \\
\hline 313 & Mana1-6Manb-Sp10 & 31 & 16 & 51 \\
\hline 432 & GlcNAcb1-2Mana1-6(GlcNAcb1-4)(GlcNAcb1-4(GlcNAcb1-2)Mana1-3)Manb1-4GlcNAcb1-4GlcNAc-Sp21 & 31 & 18 & 58 \\
\hline 87 & GalNAca1-3(Fuca1-2)Galb1-4Glcb-Sp0 & 31 & 19 & 62 \\
\hline 114 & Gala1-3Galb1-3GlcNAcb-Sp0 & 31 & 9 & 28 \\
\hline 233 & GalNAcb1-4(Neu5Aca2-3)Galb1-4Glcb-Sp0 & 31 & 11 & 37 \\
\hline 488 & Galb1-3GlcNAcb1-6GalNAca-Sp14 & 31 & 23 & 75 \\
\hline 464 & $\begin{array}{l}\text { Neu5Aca2-6Galb1-4GlcNAcb1-4Mana1-6(GlcNAcb1-4)(Neu5Aca2-6Galb1-4GlcNAcb1-4(Neu5Aca2-6Galb1- } \\
\text { 4GIcNAcb1-2)Mana1-3)Manb1-4GIcNAcb1-4GlcNAcb-Sp21 }\end{array}$ & 31 & 13 & 43 \\
\hline 93 & GalNAca1-4(Fuca1-2)Galb1-4GIcNAcb-Sp8 & 30 & 13 & 42 \\
\hline 27 & (3S)Galb1-4(6S)Glcb-Sp8 & 30 & 11 & 35 \\
\hline 551 & $\begin{array}{l}\text { Galb1-3GlcNAcb1-3Galb1-4GlcNAcb1-2Mana1-6(Galb1-3GlcNAcb1-3Galb1-4GlcNAcb1-2Mana1-3)Manb1- } \\
\text { 4GlcNAcb1-4GlcNAc-Sp25 }\end{array}$ & 30 & 8 & 26 \\
\hline 483 & $\begin{array}{l}\text { Neu5Aca2-3Galb1-4GlcNAcb1-2Mana1-6(Neu5Aca2-3Galb1-4GlcNAcb1-2Mana1-3)Manb1-4GIcNAcb1- } \\
\text { 4(Fuca1-6)GIcNAcb-Sp24 }\end{array}$ & 30 & 13 & 42 \\
\hline 182 & GlcNAcb1-3Galb1-4GlcNAcb-Sp0 & 30 & 24 & 81 \\
\hline
\end{tabular}




\begin{tabular}{|c|c|c|c|c|}
\hline 56 & $\begin{array}{l}\text { Neu5Aca2-6Galb1-4GIcNAcb1-2Mana1-6(Neu5Aca2-6Galb1-4GIcNAcb1-2Man-a1-3)Manb1-4GIcNAcb1- } \\
\text { 4GIcNAcb-Sp21 }\end{array}$ & 30 & 7 & 24 \\
\hline 277 & Galb1-3(Fuca1-4)GIcNAcb1-3Galb1-3(Fuca1-4)GlcNAcb-Sp0 & 30 & 6 & 21 \\
\hline 358 & KDNa2-3Galb1-4GIc-Sp0 & 30 & 19 & 63 \\
\hline 424 & GalNAca1-3(Fuca1-2)Galb1-3GIcNAcb1-3GalNAc-Sp14 & 29 & 15 & 52 \\
\hline 158 & Galb1-4GalNAcb1-3(Fuca1-2)Galb1-4GlcNAcb-Sp8 & 29 & 7 & 24 \\
\hline 137 & Neu5Acb2-6(Galb1-3)GalNAca-Sp8 & 29 & 16 & 55 \\
\hline 395 & $\begin{array}{l}\text { Gala1-3Galb1-3(Fuca1-4)GlcNAcb1-2Mana1-6(Gala1-3Galb1-3(Fuca1-4)GlcNAcb1-2Mana1-3)Manb1- } \\
\text { 4GIcNAcb1-4GlcNAc-Sp19 }\end{array}$ & 29 & 14 & 50 \\
\hline 544 & $\begin{array}{l}\text { Fuca1-2Galb1-4GIcNAcb1-3Galb1-4GIcNAcb1-2Mana1-6(Fuca1-2Galb1-4GIcNAcb1-3Galb1-4GIcNAcb1- } \\
\text { 2Mana1-3)Manb1-4GlcNAcb1-4GlcNAcb-Sp24 }\end{array}$ & 29 & 6 & 20 \\
\hline 180 & GlcNAcb1-3GalNAca-Sp14 & 29 & 25 & 89 \\
\hline 1 & Gala-Sp8 & 28 & 19 & 68 \\
\hline 604 & GlcNAcb1-6(Neu5Aca2-3Galb1-3)GalNAca-Sp14 & 28 & 15 & 53 \\
\hline 403 & Gala1-4Galb1-3GlcNAcb1-2Mana1-6(Gala1-4Galb1-3GlcNAcb1-2Mana1-3)Manb1-4GIcNAcb1-4GlcNAcb-Sp19 & 28 & 20 & 71 \\
\hline 223 & Neu5Aca2-3Galb1-3GalNAca-Sp8 & 28 & 17 & 59 \\
\hline 453 & GalNAcb1-4Galb1-4Glcb-Sp0 & 28 & 8 & 29 \\
\hline 478 & Neu5Aca2-3Galb1-4GIcNAcb1-2Mana-Sp0 & 28 & 13 & 48 \\
\hline 490 & Galb1-3(Fuca1-4)GIcNAcb1-6GalNAca-Sp14 & 28 & 21 & 76 \\
\hline 549 & $\begin{array}{l}\text { GlcNAcb1-3Galb1-4GIcNAcb1-3Galb1-4GIcNAcb1-3Galb1-4GIcNAcb1-2Mana1-6(GlcNAcb1-3Galb1- } \\
\text { 4GIcNAcb1-3Galb1-4GlcNAcb1-3Galb1-4GIcNAcb1-2Mana1-3)Manb1-4GIcNAcb1-4GlcNAcb-Sp25 }\end{array}$ & 28 & 4 & 15 \\
\hline 253 & Neu5Aca2-3Galb1-4(Fuca1-3)GlcNAcb1-3Galb1-4(Fuca1-3)GlcNAcb1-3Galb1-4(Fuca1-3)GlcNAcb-Sp0 & 28 & 20 & 72 \\
\hline 370 & $\begin{array}{l}\text { GalNAca1-3(Fuca1-2)Galb1-4GlcNAcb1-2Mana1-6(GalNAca1-3(Fuca1-2)Galb1-4GIcNAcb1-2Mana1-3)Manb1- } \\
\text { 4GIcNAcb1-4GIcNAcb-Sp20 }\end{array}$ & 28 & 10 & 38 \\
\hline 409 & Gala1-3(Fuca1-2)Galb1-4(Fuca1-3)Glcb-Sp21 & 27 & 10 & 35 \\
\hline 7 & Fuca-Sp9 & 27 & 14 & 50 \\
\hline 99 & GalNAcb1-4GlcNAcb-Sp8 & 27 & 12 & 42 \\
\hline 39 & $(6 S)(4 S) G a l b 1-4 G l c N A c b-S p 0$ & 27 & 7 & 26 \\
\hline 455 & $\begin{array}{l}\text { Gala1-3(Fuca1-2)Galb1-3GIcNAcb1-2Mana1-6(Gala1-3(Fuca1-2)Galb1-3GlcNAcb1-2Mana1-3)Manb1- } \\
\text { 4GIcNAcb1-4(Fuca1-6)GlcNAcb-Sp22 }\end{array}$ & 27 & 11 & 40 \\
\hline 259 & Neu5Aca2-3Galb1-4GlcNAcb-Sp0 & 27 & 7 & 24 \\
\hline 276 & Neu5Aca2-8Neu5Aca2-3Galb1-4Glcb-Sp0 & 27 & 14 & 53 \\
\hline 279 & Neu5Acb2-6Galb1-4GlcNAcb-Sp8 & 27 & 20 & 74 \\
\hline 364 & Galb1-4GlcNAcb1-2Mana1-6(Mana1-3)Manb1-4GlcNAcb1-4GlcNAcb-Sp12 & 27 & 11 & 41 \\
\hline 386 & Galb1-3GlcNAcb1-3Galb1-4(Fuca1-3)GlcNAcb1-6(Galb1-3GIcNAcb1-3)Galb1-4Glc-Sp21 & 27 & 24 & 88 \\
\hline 350 & Galb1-4GIcNAcb1-2Mana1-6Manb1-4GIcNAcb1-4GIcNAc-Sp12 & 27 & 20 & 76 \\
\hline 71 & Fuca1-2Galb1-4(Fuca1-3)GlcNAcb-Sp0 & 26 & 14 & 51 \\
\hline 97 & GalNAcb1-4(Fuca1-3)GIcNAcb-Sp0 & 26 & 4 & 14 \\
\hline 384 & Galb1-4GIcNAcb1-6(Fuca1-4(Fuca1-2Galb1-3)GlcNAcb1-3)Galb1-4Glc-Sp21 & 26 & 8 & 29 \\
\hline 89 & GalNAca1-3(Fuca1-2)Galb-Sp8 & 26 & 11 & 42 \\
\hline 546 & $\begin{array}{l}\text { GlcNAcb1-3Galb1-4GlcNAcb1-3Galb1-4GIcNAcb1-2Mana1-6(GIcNAcb1-3Galb1-4GlcNAcb1-3Galb1- } \\
\text { 4GlcNAcb1-2Mana1-3)Manb1-4GIcNAcb1-4GIcNAcb-Sp25 }\end{array}$ & 26 & 14 & 54 \\
\hline 342 & GIcNAca1-4Galb1-4GIcNAcb1-3Galb1-4(Fuca1-3)GIcNAcb1-3Galb1-4(Fuca1-3)GIcNAcb-Sp0 & 26 & 16 & 60 \\
\hline
\end{tabular}




\begin{tabular}{|c|c|c|c|c|}
\hline 34 & (3S)Galb1-4(6S)GIcNAcb-Sp0 & 26 & 18 & 71 \\
\hline 451 & GalNAca1-3(Fuca1-2)Galb1-4GIcNAcb1-6(GalNAca1-3(Fuca1-2)Galb1-4GIcNAcb1-3)GalNAc-Sp14 & 26 & 5 & 19 \\
\hline 545 & $\begin{array}{l}\text { GlcNAcb1-3Galb1-4GIcNAcb1-3Galb1-4GIcNAcb1-2Mana1-6(GIcNAcb1-3Galb1-4GIcNAcb1-3Galb1- } \\
\text { 4GIcNAcb1-2Mana1-3)Manb1-4GIcNAcb1-4GIcNAcb-Sp12 }\end{array}$ & 26 & 7 & 28 \\
\hline 470 & Glca1-4Glca1-4GIca1-4GIcb-Sp10 & 26 & 10 & 40 \\
\hline 599 & Galb1-4GIcNAcb1-3Galb1-3GalNAca-Sp14 & 26 & 7 & 29 \\
\hline 181 & GlcNAcb1-3Galb-Sp8 & 26 & 8 & 30 \\
\hline 273 & Neu5Aca2-6Galb1-4Glcb-Sp8 & 26 & 7 & 28 \\
\hline 229 & Neu5Aca2-8Neu5Aca2-8Neu5Aca-Sp8 & 26 & 13 & 53 \\
\hline 35 & (3S)Galb1-4(6S)GIcNAcb-Sp8 & 26 & 6 & 25 \\
\hline 332 & Neu5Aca2-6Galb1-4GIcNAcb1-3Galb1-4GIcNAcb1-3Galb1-4GIcNAcb-Sp0 & 25 & 9 & 34 \\
\hline 507 & $\begin{array}{l}\text { GlcNAcb1-6(GlcNAcb1-2)Mana1-6(GlcNAcb1-4)(GlcNAcb1-4(GlcNAcb1-2)Mana1-3)Manb1-4GIcNAcb1- } \\
\text { 4(Fuca1-6)GlcNAc-Sp21 }\end{array}$ & 25 & 16 & 64 \\
\hline 297 & (6S)Galb1-4(6S)GIcNAcb-Sp0 & 25 & 18 & 70 \\
\hline 467 & Gala1-3(Fuca1-2)Galb1-3GalNAca-Sp8 & 25 & 22 & 88 \\
\hline 475 & GlcNAcb1-6(GlcNAcb1-2)Mana1-6(GlcNAcb1-2Mana1-3)Manb1-4GIcNAcb1-4(Fuca1-6)GlcNAcb-Sp24 & 25 & 6 & 24 \\
\hline 531 & $\begin{array}{l}\text { Galb1-4GIcNAcb1-2 Mana1-6(GlcNAcb1-4)(Galb1-4GIcNAcb1-2Mana1-3)Manb1-4GlcNAcb1-4(Fuca1- } \\
\text { 6)GlcNAc-Sp21 }\end{array}$ & 25 & 4 & 17 \\
\hline 415 & GalNAca1-3GalNAcb1-3Gala1-4Galb1-4Glcb-Sp0 & 25 & 17 & 69 \\
\hline 144 & Galb1-3GalNAcb1-4(Neu5Aca2-3)Galb1-4GIcb-Sp0 & 25 & 13 & 53 \\
\hline 156 & Galb1-4(6S)Glcb-Sp8 & 25 & 7 & 30 \\
\hline 609 & GlcNAcb1-3Fuca-Sp21 & 25 & 5 & 19 \\
\hline 355 & (6S)GlcNAcb1-3Galb1-4GlcNAcb-Sp0 & 25 & 15 & 59 \\
\hline 388 & GlcNAcb1-2Mana1-6(GlcNAcb1-4(GIcNAcb1-2)Mana1-3)Manb1-4GIcNAcb1-4GIcNAc-Sp21 & 25 & 13 & 53 \\
\hline 524 & Galb1-3GIcNAcb1-2Mana-Sp0 & 25 & 12 & 50 \\
\hline 591 & Galb1-4GIcNAcb1-3Galb1-4GIcNAcb1-6(Galb1-4GIcNAcb1-3Galb1-4GIcNAcb1-3)GalNAca-Sp14 & 25 & 12 & 50 \\
\hline 88 & GlcNAcb1-3Galb1-3GalNAca-Sp8 & 24 & 5 & 20 \\
\hline 9 & Neu5Aca-Sp8 & 24 & 14 & 58 \\
\hline 527 & Gala1-3Galb1-3GlcNAcb1-2Mana-Sp0 & 24 & 4 & 18 \\
\hline 417 & Gala1-3(Fuca1-2)Galb1-4(Fuca1-3)GlcNAcb1-3GalNAc-Sp14 & 24 & 13 & 55 \\
\hline 392 & GalNAcb1-4(Neu5Aca2-3)Galb1-4GlcNAcb1-3GalNAca-Sp14 & 24 & 13 & 55 \\
\hline 121 & Gala1-4Galb1-4GlcNAcb-Sp8 & 24 & 7 & 28 \\
\hline 308 & Neu5Aca2-6Galb1-4GIcNAcb1-2Mana1-6(GIcNAcb1-2Mana1-3)Manb1-4GIcNAcb1-4GIcNAcb-Sp12 & 24 & 5 & 20 \\
\hline 111 & Gala1-3GalNAca-Sp16 & 24 & 10 & 43 \\
\hline 22 & $6 S(3 S) G a l b 1-4(6 S) G l c N A c b-S p 0$ & 24 & 4 & 15 \\
\hline 511 & $(6 S)(4 S) G a l N A c b 1-4 G l c N A c-S p 8$ & 24 & 4 & 16 \\
\hline 13 & Glcb-Sp8 & 24 & 6 & 27 \\
\hline 498 & Gala1-3(Fuca1-2)Galb1-4GIcNAcb1-6GalNAca-Sp14 & 24 & 13 & 55 \\
\hline 473 & $\begin{array}{l}\text { Fuca1-2Galb1-3(Fuca1-4)GlcNAcb1-2Mana1-6(Fuca1-2Galb1-3(Fuca1-4)GlcNAcb1-2Mana1-3)Manb1- } \\
\text { 4GlcNAcb1-4(Fuca1-6)GlcNAcb1-4(Fuca1-6)GlcNAcb-Sp19 }\end{array}$ & 24 & 6 & 26 \\
\hline 602 & Neu5Aca2-6Galb1-4GIcNAcb1-6(Galb1-3)GalNAca-Sp14 & 24 & 1 & 4 \\
\hline 412 & Neu5Aca2-3Galb1-3GalNAcb1-4(Neu5Aca2-8Neu5Aca2-3)Galb1-4Glcb-Sp0 & 24 & 16 & 69 \\
\hline 366 & Neu5Aca2-6GlcNAcb1-4GlcNAc-Sp21 & 24 & 9 & 38 \\
\hline
\end{tabular}




\begin{tabular}{|c|c|c|c|c|}
\hline 575 & $\begin{array}{l}\text { Galb1-4GIcNAcb1-3Galb1-4GIcNAcb1-2Mana1-6(Galb1-4GIcNAcb1-3Galb1-4GIcNAcb1-2Mana1-3)Manb1- } \\
\text { 4GlcNAcb1-4(Fuca1-6)GlcNAcb-Sp24 }\end{array}$ & 24 & 9 & 37 \\
\hline 311 & GIcNAcb1-4GIcNAcb-Sp12 & 23 & 10 & 41 \\
\hline 302 & Galb1-4GIcNAcb1-6(Galb1-4GIcNAcb1-3)Galb1-4GlcNAc-Sp0 & 23 & 15 & 64 \\
\hline 312 & MurNAcb1-4GlcNAcb-Sp10 & 23 & 11 & 47 \\
\hline 25 & (3S)Galb1-4Glcb-Sp8 & 23 & 11 & 48 \\
\hline 422 & Fuca1-2Galb1-3GIcNAcb1-3GalNAc-Sp14 & 23 & 23 & 99 \\
\hline 421 & GIcNAcb1-2(GlcNAcb1-6)Mana1-6(GlcNAcb1-2Mana1-3)Manb1-4GIcNAcb1-4GIcNAcb-Sp19 & 23 & 16 & 69 \\
\hline 359 & KDNa2-3Galb1-3GalNAca-Sp14 & 23 & 17 & 73 \\
\hline 443 & (6S)Galb1-3GlcNAcb-Sp0 & 23 & 17 & 74 \\
\hline 425 & Gala1-3Galb1-3GlcNAcb1-3GalNAc-Sp14 & 23 & 4 & 19 \\
\hline 540 & $\begin{array}{l}\text { GlcNAcb1-3Galb1-4GlcNAcb1-2Mana1-6(GIcNAcb1-3Galb1-4GIcNAcb1-2Mana1-3)Manb1-4GIcNAcb1- } \\
\text { 4GIcNAcb-Sp25 }\end{array}$ & 23 & 9 & 41 \\
\hline 360 & $\begin{array}{l}\text { Fuca1-2Galb1-3GIcNAcb1-2Mana1-6(Fuca1-2Galb1-3GlcNAcb1-2Mana1-3)Manb1-4GIcNAcb1-4GIcNAcb- } \\
\text { Sp20 }\end{array}$ & 23 & 10 & 44 \\
\hline 120 & Gala1-4Galb1-4GIcNAcb-Sp0 & 23 & 14 & 63 \\
\hline 436 & $\begin{array}{l}\text { Galb1-4GIcNAcb1-2Mana1-6(GIcNAcb1-4)(Galb1-4GIcNAcb1-4(Galb1-4GIcNAcb1-2)Mana1-3)Manb1- } \\
\text { 4GIcNAcb1-4GIcNAc-Sp21 }\end{array}$ & 23 & 14 & 61 \\
\hline 248 & Fuca1-2(6S)Galb1-4GIcb-Sp0 & 23 & 14 & 62 \\
\hline 400 & Fuca1-2Galb1-4GIcNAcb1-3GalNAca-Sp14 & 23 & 14 & 62 \\
\hline 314 & Mana1-6(Mana1-3)Mana1-6(Mana1-3)Manb-Sp10 & 22 & 14 & 63 \\
\hline 405 & Gala1-3Galb1-4GIcNAcb1-3GalNAca-Sp14 & 22 & 16 & 71 \\
\hline 124 & Gala1-6Glcb-Sp8 & 22 & 12 & 56 \\
\hline 550 & $\begin{array}{l}\text { Galb1-4GlcNAcb1-3Galb1-4GIcNAcb1-3Galb1-4GIcNAcb1-3Galb1-4GIcNAcb1-2Mana1-6(Galb1-4GlcNAcb1- } \\
\text { 3Galb1-4GlcNAcb1-3Galb1-4GIcNAcb1-3Galb1-4GIcNAcb1-2Mana1-3)Manb1-4GIcNAcb1-4GIcNAcb-Sp25 }\end{array}$ & 22 & 2 & 11 \\
\hline 81 & Fucb1-3GIcNAcb-Sp8 & 22 & 9 & 41 \\
\hline 247 & Neu5Aca2-3Galb1-3GIcNAcb1-3Galb1-4GIcNAcb-Sp0 & 22 & 5 & 21 \\
\hline 494 & Fuca1-2Galb1-4GIcNAcb1-6GalNAca-Sp14 & 22 & 16 & 74 \\
\hline 394 & Gala1-3Galb1-3GIcNAcb1-2Mana1-6(Gala1-3Galb1-3GIcNAcb1-2Mana1-3)Manb1-4GIcNAcb1-4GIcNAc-Sp19 & 22 & 6 & 30 \\
\hline 226 & GalNAcb1-4(Neu5Aca2-8Neu5Aca2-8Neu5Aca2-3)Galb1-4Glcb-Sp0 & 22 & 14 & 64 \\
\hline 38 & (3S)Galb-Sp8 & 22 & 8 & 37 \\
\hline 547 & $\begin{array}{l}\text { Galb1-4GlcNAcb1-3Galb1-4GIcNAcb1-3Galb1-4GIcNAcb1-2Mana1-6(Galb1-4GIcNAcb1-3Galb1-4GlcNAcb1- } \\
\text { 3Galb1-4GIcNAcb1-2Mana1-3)Manb1-4GIcNAcb1-4GIcNAcb-Sp12 }\end{array}$ & 21 & 23 & 110 \\
\hline 564 & $\begin{array}{l}\text { GlcNAcb1-3Galb1-4GIcNAcb1-3Galb1-4GIcNAcb1-3Galb1-4GIcNAcb1-3Galb1-4GIcNAcb1-2Mana1- } \\
\text { 6(GlcNAcb1-3Galb1-4GIcNAcb1-3Galb1-4GIcNAcb1-3Galb1-4GIcNAcb1-3Galb1-4GIcNAcb1-2Mana1- } \\
\text { 3)Manb1-4GIcNAcb1-4GlcNAcb-Sp25 }\end{array}$ & 21 & 5 & 23 \\
\hline 103 & Gala1-3(Fuca1-2)Galb1-4(Fuca1-3)GlcNAcb-Sp0 & 21 & 8 & 39 \\
\hline 566 & GlcNAb1-3Galb1-3GalNAc-Sp14 & 21 & 6 & 30 \\
\hline 529 & Neu5Aca2-3Galb1-3GalNAcb1-4Galb1-4GIcb-Sp0 & 21 & 8 & 38 \\
\hline 58 & Fuca1-2Galb1-3GalNAcb1-3Gala-Sp9 & 21 & 10 & 47 \\
\hline 239 & Neu5Aca2-3Galb1-3(Fuca1-4)GIcNAcb-Sp8 & 21 & 10 & 47 \\
\hline 517 & Galb1-4(6P)GlcNAcb-Sp0 & 21 & 18 & 86 \\
\hline 190 & GlcNAcb1-4GIcNAcb1-4GIcNAcb1-4GIcNAcb1-4GlcNAcb1-Sp8 & 21 & 8 & 40 \\
\hline
\end{tabular}




\begin{tabular}{|c|c|c|c|c|}
\hline 500 & Fuca1-2Galb1-3(6S)GIcNAcb-Sp0 & 21 & 14 & 68 \\
\hline 280 & Neu5Gca2-3Galb1-3(Fuca1-4)GIcNAcb-Sp0 & 21 & 16 & 77 \\
\hline 238 & Neu5Aca2-3Galb1-3(6S)GIcNAc-Sp8 & 21 & 6 & 31 \\
\hline 375 & $\begin{array}{l}\text { Fuca1-4(Fuca1-2Galb1-3)GIcNAcb1-2Mana1-3(Fuca1-4(Fuca1-2Galb1-3)GlcNAcb1-2Mana1-3)Manb1- } \\
\text { 4GIcNAcb1-4GIcNAcb-Sp19 }\end{array}$ & 21 & 8 & 39 \\
\hline 596 & $\begin{array}{l}\text { Neu5Aca2-3Galb1-4GIcNAcb1-3Galb1-4GIcNAcb1-6(Neu5Aca2-3Galb1-4GIcNAcb1-3Galb1-4GIcNAcb1- } \\
\text { 3)GalNAca-Sp14 }\end{array}$ & 20 & 9 & 46 \\
\hline 126 & Galb1-3(Fuca1-4)GlcNAcb1-3Galb1-4(Fuca1-3)GIcNAcb-Sp0 & 20 & 14 & 69 \\
\hline 369 & $\begin{array}{l}\text { Galb1-4GIcNAcb1-2Mana1-6(Galb1-4GlcNAcb1-4(Galb1-4GlcNAcb1-2)Mana1-3)Manb1-4GIcNAcb1-4GIcNAc- } \\
\text { Sp21 }\end{array}$ & 20 & 10 & 50 \\
\hline 480 & Neu5Aca2-6Galb1-4GIcNAcb1-6GalNAca-Sp14 & 20 & 8 & 37 \\
\hline 331 & Neu5Aca2-3Galb1-3(Fuca1-4)GIcNAcb1-3Galb1-3(Fuca1-4)GIcNAcb-Sp0 & 20 & 16 & 81 \\
\hline 159 & Galb1-4GIcNAcb1-3GalNAca-Sp8 & 20 & 7 & 34 \\
\hline 423 & Gala1-3(Fuca1-2)Galb1-3GIcNAcb1-3GalNAc-Sp14 & 20 & 18 & 88 \\
\hline 45 & (6S)Galb1-4(6S)Glcb-Sp8 & 20 & 19 & 93 \\
\hline 183 & GlcNAcb1-3Galb1-4GlcNAcb-Sp8 & 20 & 6 & 31 \\
\hline 52 & GlcNAcb1-2Mana1-6(GlcNAcb1-2Mana1-3)Manb1-4GlcNAcb1-4GlcNAcb-Sp12 & 20 & 9 & 46 \\
\hline 292 & Galb1-4(Fuca1-3)GlcNAcb1-3Galb1-3(Fuca1-4)GlcNAcb-Sp0 & 20 & 7 & 35 \\
\hline 552 & Neu5Gca2-8Neu5Gca2-3Galb1-4GIcNAc-Sp0 & 20 & 4 & 20 \\
\hline 428 & $\begin{array}{l}\text { Galb1-3GIcNAcb1-6(Galb1-3GIcNAcb1-2)Mana1-6(Galb1-3GlcNAcb1-2Mana1-3)Manb1-4GIcNAcb1- } \\
\text { 4GIcNAcb-Sp19 }\end{array}$ & 20 & 4 & 18 \\
\hline 109 & Gala1-4(Gala1-3)Galb1-4GIcNAcb-Sp8 & 20 & 13 & 65 \\
\hline 448 & Galb1-4GlcNAcb1-2Mana-Sp0 & 20 & 6 & 29 \\
\hline 601 & Neu5Aca2-6Galb1-4GIcNAcb1-3Galb1-4GIcNAcb1-6(Galb1-3)GalNAca-Sp14 & 20 & 6 & 32 \\
\hline 48 & $\mathrm{Neu} 5,9 \mathrm{Ac}_{2} \mathrm{a}-\mathrm{Sp} 8$ & 20 & 20 & 102 \\
\hline 132 & Galb1-4GIcNAcb1-6GalNAc-Sp14 & 20 & 13 & 67 \\
\hline 310 & GlcNAcb1-4GlcNAcb-Sp10 & 20 & 12 & 59 \\
\hline 254 & Neu5Aca2-3Galb1-4(Fuca1-3)GlcNAcb-Sp0 & 20 & 6 & 32 \\
\hline 520 & Neu5Aca2-6Galb1-4GIcNAcb1-2Man-Sp0 & 20 & 8 & 43 \\
\hline 445 & $\begin{array}{l}\text { Fuca1-2Galb1-4 GlcNAcb1-2Mana1-6(Fuca1-2Galb1-4GIcNAcb1-2(Fuca1-2Galb1-4GlcNAcb1-4)Mana1- } \\
\text { 3)Manb1-4GIcNAcb1-4GIcNAcb-Sp12 }\end{array}$ & 20 & 5 & 27 \\
\hline 610 & Galb1-3GalNAcb1-4(Neu5Aca2-8Neu5Aca2-8Neu5Aca2-3)Galb1-4Glcb-Sp21 & 19 & 23 & 116 \\
\hline 371 & $\begin{array}{l}\text { Gala1-3(Fuca1-2)Galb1-4GlcNAcb1-2Mana1-6(Gala1-3(Fuca1-2)Galb1-4GIcNAcb1-2Mana1-3)Manb1- } \\
\text { 4GIcNAcb1-4GIcNAcb-Sp20 }\end{array}$ & 19 & 19 & 98 \\
\hline 548 & $\begin{array}{l}\text { Galb1-4GIcNAcb1-3Galb1-4GIcNAcb1-3Galb1-4GIcNAcb1-2Mana1-6(Galb1-4GIcNAcb1-3Galb1-4GIcNAcb1- } \\
\text { 3Galb1-4GIcNAcb1-2Mana1-3)Manb1-4GIcNAcb1-4GIcNAcb-Sp24 }\end{array}$ & 19 & 8 & 42 \\
\hline 373 & $\begin{array}{l}\text { GalNAca1-3(Fuca1-2)Galb1-3GIcNAcb1-2Mana1-6(GalNAca1-3(Fuca1-2)Galb1-3GlcNAcb1-2Mana1-3)Manb1- } \\
\text { 4GlcNAcb1-4GlcNAcb-Sp20 }\end{array}$ & 19 & 10 & 53 \\
\hline 571 & $\begin{array}{l}\text { Galb1-3GlcNAcb1-3Galb1-4GIcNAcb1-3Galb1-4GIcNAcb1-6(Galb1-3GIcNAcb1-3Galb1-4GlcNAcb1-3Galb1- } \\
\text { 4GIcNAb1-2)Mana1-6(Galb1-3GlcNAcb1-3Galb1-4GIcNAcb1-3Galb1-4GIcNAcb1-2Mana1-3)Manb1- } \\
\text { 4GIcNAcb1-4(Fuca1-6)GlcNAcb-Sp24 }\end{array}$ & 19 & 4 & 20 \\
\hline 11 & Neu5Acb-Sp8 & 19 & 5 & 28 \\
\hline 116 & Gala1-3Galb1-4Glcb-Sp0 & 19 & 15 & 77 \\
\hline
\end{tabular}




\begin{tabular}{|c|c|c|c|c|}
\hline 411 & Galb1-3GalNAcb1-4(Neu5Aca2-8Neu5Aca2-3)Galb1-4Glcb-Sp0 & 19 & 6 & 33 \\
\hline 382 & Galb1-3GIcNAcb1-3Galb1-4GIcNAcb1-6(Galb1-3GIcNAcb1-3)Galb1-4Glcb-Sp0 & 19 & 5 & 27 \\
\hline 251 & Neu5Aca2-3Galb1-4(6S)GlcNAcb-Sp8 & 19 & 10 & 51 \\
\hline 104 & Gala1-3(Fuca1-2)Galb1-4(Fuca1-3)GIcNAcb-Sp8 & 19 & 9 & 45 \\
\hline 105 & Gala1-3(Fuca1-2)Galb1-4GlcNAc-Sp0 & 19 & 12 & 61 \\
\hline 263 & Neu5Aca2-3Galb1-4Glcb-Sp0 & 19 & 12 & 65 \\
\hline 53 & GIcNAcb1-2Mana1-6(GIcNAcb1-2Mana1-3)Manb1-4GIcNAcb1-4GIcNAcb-Sp13 & 19 & 18 & 97 \\
\hline 282 & Neu5Gca2-3Galb1-4(Fuca1-3)GlcNAcb-Sp0 & 19 & 12 & 65 \\
\hline 218 & Neu5Aca2-3Galb1-4GIcNAcb1-3Galb1-4(Fuca1-3)GIcNAcb-Sp0 & 19 & 7 & 38 \\
\hline 68 & Fuca1-2Galb1-3GIcNAcb-Sp8 & 19 & 8 & 41 \\
\hline 340 & GIcNAca1-4Galb1-3GIcNAcb-Sp0 & 19 & 18 & 98 \\
\hline 536 & GalNAca1-3(Fuca1-2)Galb1-3GalNAcb1-3Gala1-4Galb1-4Glc-Sp21 & 19 & 8 & 45 \\
\hline 6 & Fuca-Sp8 & 19 & 10 & 55 \\
\hline 456 & Neu5Aca2-6Galb1-4GIcNAcb1-6(Fuca1-2Galb1-3GIcNAcb1-3)Galb1-4GIc-Sp21 & 19 & 13 & 71 \\
\hline 31 & (3S)Galb1-3GlcNAcb-Sp8 & 19 & 9 & 49 \\
\hline 26 & (3S)Galb1-4(6S)Glcb-Sp0 & 19 & 21 & 114 \\
\hline 446 & $\begin{array}{l}\text { Fuca1-2Galb1-4(Fuca1-3)GlcNAcb1-2Mana1-6(Fuca1-2Galb1-4(Fuca1-3)GlcNAcb1-4(Fuca1-2Galb1-4(Fuca1- } \\
\text { 3)GIcNAcb1-2)Mana1-3)Manb1-4GlcNAcb1-4GlcNAcb-Sp12 }\end{array}$ & 18 & 8 & 44 \\
\hline 149 & Galb1-3GIcNAcb-Sp0 & 18 & 22 & 122 \\
\hline 543 & $\begin{array}{l}\text { Neu5Gca2-3Galb1-4GIcNAcb1-3Galb1-4GlcNAcb1-2Mana1-6(Neu5Gca2-3Galb1-4GlcNAcb1-3Galb1- } \\
\text { 4GlcNAcb1-2Mana1-3)Manb1-4GlcNAcb1-4GlcNAcb-Sp24 }\end{array}$ & 18 & 4 & 21 \\
\hline 234 & Neu5Aca2-3Galb1-3GalNAcb1-4(Neu5Aca2-3)Galb1-4Glcb-Sp0 & 18 & 10 & 54 \\
\hline 141 & Galb1-3GalNAca-Sp16 & 18 & 7 & 40 \\
\hline 450 & Gala1-3(Fuca1-2)Galb1-4GlcNAcb1-6(Gala1-3(Fuca1-2)Galb1-4GlcNAcb1-3)GalNAc-Sp14 & 18 & 4 & 19 \\
\hline 90 & GalNAca1-3(Fuca1-2)Galb-Sp18 & 18 & 29 & 161 \\
\hline 447 & Galb1-4(Fuca1-3)GlcNAcb1-6GaINAc-Sp14 & 18 & 5 & 30 \\
\hline 153 & Galb1-4(Fuca1-3)GIcNAcb1-3Galb1-4(Fuca1-3)GIcNAcb-Sp0 & 18 & 13 & 71 \\
\hline 328 & Neu5,9Ac2a2-3Galb1-4GIcNAcb-Sp0 & 18 & 7 & 37 \\
\hline 83 & GalNAca1-3(Fuca1-2)Galb1-4(Fuca1-3)GlcNAcb-Sp0 & 18 & 10 & 56 \\
\hline 444 & (6S)Galb1-3(6S)GlcNAc-Sp0 & 18 & 5 & 29 \\
\hline 134 & GIcNAcb1-6(Galb1-3)GalNAca-Sp14 & 18 & 5 & 30 \\
\hline 581 & $\begin{array}{l}\text { Galb1-4GIcNAcb1-3Galb1-4GIcNAcb1-3Galb1-4GlcNAcb1-3Galb1-4GIcNAcb1-3Galb1-4GIcNAcb1-2Mana1- } \\
\text { 6(Galb1-4GIcNAcb1-3Galb1-4GIcNAcb1-3Galb1-4GIcNAcb1-3Galb1-4GIcNAcb1-3Galb1-4GIcNAcb1-2Mana1- } \\
\text { 3)Manb1-4GlcNAcb1-4(Fuca1-6)GIcNAcb-Sp19 }\end{array}$ & 18 & 6 & 34 \\
\hline 236 & Neu5Aca2-3GalNAca-Sp8 & 18 & 15 & 84 \\
\hline 274 & Neu5Aca2-6Galb-Sp8 & 18 & 9 & 52 \\
\hline 429 & Galb1-4GIcNAcb1-6(Fuca1-2Galb1-3GIcNAcb1-3)Galb1-4GIc-Sp21 & 18 & 10 & 54 \\
\hline 503 & GalNAcb1-4(Fuca1-3)(6S)GlcNAcb-Sp8 & 18 & 6 & 34 \\
\hline 54 & Galb1-4GlcNAcb1-2Mana1-6(Galb1-4GlcNAcb1-2Mana1-3)Manb1-4GlcNAcb1-4GlcNAcb-Sp12 & 18 & 25 & 140 \\
\hline 33 & (3S)Galb1-4(Fuca1-3)GlcNAc-Sp8 & 18 & 10 & 55 \\
\hline 154 & Galb1-4(Fuca1-3)GIcNAcb1-3Galb1-4(Fuca1-3)GIcNAcb1-3Galb1-4(Fuca1-3)GIcNAcb-Sp0 & 18 & 20 & 111 \\
\hline 535 & GlcNAcb1-3Galb1-4GIcNAcb1-6(GIcNAcb1-3)Galb1-4GlcNAc-Sp0 & 18 & 6 & 32 \\
\hline 593 & GlcNAcb1-3Galb1-4GIcNAcb1-3GalNAca-Sp14 & 18 & 9 & 54 \\
\hline
\end{tabular}




\begin{tabular}{|c|c|c|c|c|}
\hline 146 & Galb1-3Galb-Sp8 & 18 & 5 & 30 \\
\hline 55 & $\begin{array}{l}\text { Neu5Aca2-6Galb1-4GIcNAcb1-2Mana1-6(Neu5Aca2-6Galb1-4GIcNAcb1-2Mana1-3)Manb1-4GlcNAcb1- } \\
\text { 4GlcNAcb-Sp12 }\end{array}$ & 18 & 6 & 33 \\
\hline 107 & Gala1-3(Fuca1-2)Galb-Sp8 & 17 & 4 & 26 \\
\hline 306 & GalNAcb1-3Galb-Sp8 & 17 & 16 & 90 \\
\hline 343 & GlcNAca1-4Galb1-4GlcNAcb1-3Galb1-4GlcNAcb-Sp0 & 17 & 7 & 42 \\
\hline 91 & GalNAca1-3GalNAcb-Sp8 & 17 & 7 & 41 \\
\hline 472 & $\begin{array}{l}\text { Fuca1-2Galb1-4(Fuca1-3)GlcNAcb1-2Mana1-6(Fuca1-2Galb1-4(Fuca1-3)GIcNAcb1-2Mana1-3)Manb1- } \\
\text { 4GIcNAcb1-4(Fuca1-6)GIcNAcb-Sp24 }\end{array}$ & 17 & 10 & 58 \\
\hline 512 & (6S)GalNAcb1-4GlcNAc-Sp8 & 17 & 13 & 74 \\
\hline 261 & Neu5Aca2-3Galb1-4GIcNAcb1-3Galb1-4GIcNAcb-Sp0 & 17 & 6 & 35 \\
\hline 565 & $\begin{array}{l}\text { Galb1-4GlcNAcb1-3Galb1-4GIcNAcb1-3Galb1-4GIcNAcb1-3Galb1-4GIcNAcb1-3Galb1-4GIcNAcb1-2Mana1- } \\
\text { 6(Galb1-4GIcNAcb1-3Galb1-4GIcNAcb1-3Galb1-4GlcNAcb1-3Galb1-4GIcNAcb1-3Galb1-4GIcNAcb1-2Mana1- } \\
\text { 3)Manb1-4GIcNAcb1-4GlcNAcb-Sp25 }\end{array}$ & 17 & 8 & 45 \\
\hline 486 & $\begin{array}{l}\text { Neu5Aca2-3Galb1-3GlcNAcb1-2Mana1-6(GlcNAcb1-4)(Neu5Aca2-3Galb1-3GlcNAcb1-2Mana1-3)Manb1- } \\
\text { 4GIcNAcb1-4GlcNAc-Sp21 }\end{array}$ & 17 & 5 & 31 \\
\hline 118 & Gala1-3Galb-Sp8 & 17 & 9 & 50 \\
\hline 495 & Gala1-3Galb1-4GlcNAcb1-6GalNAca-Sp14 & 17 & 8 & 43 \\
\hline 336 & GalNAca1-3(Fuca1-2)Galb1-4GlcNAcb1-3Galb1-4GlcNAcb1-3Galb1-4GlcNAcb-Sp0 & 17 & 7 & 42 \\
\hline 393 & GalNAca1-3(Fuca1-2)Galb1-3GalNAca1-3(Fuca1-2)Galb1-4GlcNAcb-Sp0 & 17 & 9 & 51 \\
\hline 205 & KDNa2-3Galb1-3GIcNAcb-Sp0 & 17 & 7 & 38 \\
\hline 563 & GalNAcb1-4GIcNAcb1-3GalNAcb1-4GlcNAcb-Sp0 & 17 & 7 & 41 \\
\hline 57 & $\begin{array}{l}\text { Neu5Aca2-6Galb1-4GIcNAcb1-2Mana1-6(Neu5Aca2-6Galb1-4GIcNAcb1-2Mana1-3)Manb1-4GlcNAcb1- } \\
\text { 4GIcNAcb-Sp24 }\end{array}$ & 17 & 9 & 52 \\
\hline 534 & Neu5Aca2-3Galb1-4(Fuca1-3)GlcNAcb1-2Mana-Sp0 & 17 & 14 & 85 \\
\hline 389 & Fuca1-2Galb1-3GalNAca1-3(Fuca1-2)Galb1-4Glcb-Sp0 & 17 & 6 & 37 \\
\hline 136 & Neu5Aca2-6(Galb1-3)GalNAca-Sp14 & 17 & 14 & 86 \\
\hline 562 & GalNAcb1-3GlcNAcb-Sp0 & 17 & 10 & 57 \\
\hline 215 & Mana1-6(Mana1-3)Mana1-6(Mana1-2Mana1-3)Manb1-4GIcNAcb1-4GIcNAcb-Sp12 & 17 & 9 & 54 \\
\hline 290 & Galb1-4(Fuca1-3)(6S)GIcNAcb-Sp0 & 17 & 4 & 21 \\
\hline 44 & (6S)Galb1-4GlcNAcb-Sp8 & 17 & 12 & 72 \\
\hline 434 & $\begin{array}{l}\text { GlcNAcb1-6(GlcNAcb1-2)Mana1-6(GlcNAcb1-4)(GlcNAcb1-4(GlcNAcb1-2)Mana1-3)Manb1-4GlcNAcb1- } \\
\text { 4GIcNAc-Sp21 }\end{array}$ & 17 & 14 & 84 \\
\hline 293 & Galb1-4GIcNAcb1-3Galb1-3GIcNAcb-Sp0 & 17 & 24 & 147 \\
\hline 131 & Galb1-4GlcNAcb1-6GalNAca-Sp8 & 17 & 12 & 75 \\
\hline 525 & Gala1-3(Fuca1-2)Galb1-3GIcNAcb1-6GalNAc-Sp14 & 17 & 9 & 55 \\
\hline 542 & $\begin{array}{l}\text { Galb1-4GlcNAcb1-3Galb1-4GIcNAcb1-2Mana1-6(Galb1-4GlcNAcb1-3Galb1-4GlcNAcb1-2Mana1-3)Manb1- } \\
\text { 4GIcNAcb1-4GlcNAcb-Sp24 }\end{array}$ & 17 & 3 & 21 \\
\hline 5 & GalNAca-Sp15 & 16 & 11 & 69 \\
\hline 59 & Fuca1-2Galb1-3GalNAcb1-3Gala1-4Galb1-4Glcb-Sp9 & 16 & 13 & 78 \\
\hline 200 & G-ol-Sp8 & 16 & 10 & 64 \\
\hline 391 & Galb1-3GIcNAcb1-3GalNAca-Sp14 & 16 & 9 & 56 \\
\hline 553 & Neu5Aca2-8Neu5Gca2-3Galb1-4GlcNAc-Sp0 & 16 & 11 & 68 \\
\hline
\end{tabular}




\begin{tabular}{|c|c|c|c|c|}
\hline 192 & GlcNAcb1-6GalNAca-Sp8 & 16 & 20 & 126 \\
\hline 307 & GlcAb1-3GlcNAcb-Sp8 & 16 & 8 & 47 \\
\hline 501 & Fuca1-2(6S)Galb1-3(6S)GlcNAcb-Sp0 & 16 & 8 & 47 \\
\hline 230 & Neu5Aca2-3(6S)Galb1-4(Fuca1-3)GlcNAcb-Sp8 & 16 & 11 & 67 \\
\hline 324 & Galb1-3GIcNAcb1-2Mana1-6(Galb1-3GlcNAcb1-2Mana1-3)Manb1-4GIcNAcb1-4GIcNAcb-Sp19 & 16 & 11 & 69 \\
\hline 122 & Gala1-4Galb1-4Glcb-Sp0 & 16 & 10 & 60 \\
\hline 560 & Gala1-3Galb1-4GIcNAcb1-2Mana1-6(Gala1-3Galb1-4GIcNAcb1-2Mana1-3)Manb1-4GIcNAcb1-4GIcNAc-Sp24 & 16 & 7 & 41 \\
\hline 383 & Galb1-4(Fuca1-3)GIcNAcb1-6(Galb1-3GIcNAcb1-3)Galb1-4GIc-Sp21 & 16 & 8 & 53 \\
\hline 167 & Galb1-4GIcNAcb1-6(Galb1-3)GalNAc-Sp14 & 16 & 6 & 39 \\
\hline 338 & GlcNAca1-4Galb1-4GlcNAcb1-3Galb1-4GlcNAcb1-3Galb1-4GIcNAcb-Sp0 & 16 & 10 & 64 \\
\hline 77 & Fuca1-2Galb1-4Glcb-Sp0 & 16 & 9 & 55 \\
\hline 72 & Fuca1-2Galb1-4(Fuca1-3)GlcNAcb-Sp8 & 16 & 9 & 57 \\
\hline 419 & $\begin{array}{l}\text { Galb1-4(Fuca1-3)GlcNAcb1-2Mana1-6(Galb1-4(Fuca1-3)GlcNAcb1-2Mana1-3)Manb1-4GlcNAcb1-4(Fuca1- } \\
\text { 6)GlcNAcb-Sp22 }\end{array}$ & 16 & 6 & 41 \\
\hline 530 & GlcNAcb1-2 Mana1-6(GlcNAcb1-4)(GlcNAcb1-2Mana1-3)Manb1-4GlcNAcb1-4(Fuca1-6)GIcNAc-Sp21 & 16 & 7 & 43 \\
\hline 496 & Galb1-4(Fuca1-3)GlcNAcb1-2Mana-Sp0 & 16 & 6 & 41 \\
\hline 518 & (6P)Galb1-4GlcNAcb-SP0 & 16 & 10 & 63 \\
\hline 92 & GalNAca1-3Galb-Sp8 & 15 & 16 & 105 \\
\hline 219 & (3S)Galb1-4(Fuca1-3)(6S)GlcNAcb-Sp8 & 15 & 7 & 44 \\
\hline 241 & Neu5Aca2-3Galb1-4(Neu5Aca2-3Galb1-3)GlcNAcb-Sp8 & 15 & 20 & 127 \\
\hline 171 & Galb1-4GIcb-Sp0 & 15 & 10 & 67 \\
\hline 242 & Neu5Aca2-3Galb1-3(6S)GalNAca-Sp8 & 15 & 14 & 93 \\
\hline 69 & Fuca1-2Galb1-4(Fuca1-3)GlcNAcb1-3Galb1-4(Fuca1-3)GIcNAcb-Sp0 & 15 & 4 & 27 \\
\hline 207 & Mana1-2Mana1-2Mana1-3Mana-Sp9 & 15 & 15 & 99 \\
\hline 139 & Galb1-3GalNAca-Sp8 & 15 & 5 & 30 \\
\hline 357 & KDNa2-6Galb1-4GIcNAc-Sp0 & 15 & 4 & 26 \\
\hline 559 & $\begin{array}{l}\text { Galb1-4GIcNAcb1-3Galb1-4GIcNAcb1-6(Galb1-4GIcNAcb1-3Galb1-4GIcNAcb1-2)Mana1-6(Galb1-4GIcNAcb1- } \\
\text { 3Galb1-4GIcNAcb1-2Mana1-3)Mana1-4GIcNAcb1-4GIcNAc-Sp24 }\end{array}$ & 15 & 13 & 83 \\
\hline 316 & Mana1-2Mana1-6(Mana1-2Mana1-3)Mana1-6(Mana1-2Mana1-2Mana1-3)Mana-Sp9 & 15 & 5 & 30 \\
\hline 322 & Neu5Aca2-8Neu5Aca2-8Neu5Acb-Sp8 & 15 & 27 & 182 \\
\hline 240 & Neu5Aca2-3Galb1-3(Fuca1-4)GlcNAcb1-3Galb1-4(Fuca1-3)GIcNAcb-Sp0 & 15 & 7 & 49 \\
\hline 387 & $\begin{array}{l}\text { Galb1-4GIcNAcb1-6(Galb1-4GIcNAcb1-2)Mana1-6(Galb1-4GIcNAcb1-4(Galb1-4GlcNAcb1-2)Mana1-3)Manb1- } \\
\text { 4GIcNAcb1-4GIcNAcb-Sp21 }\end{array}$ & 15 & 16 & 112 \\
\hline 19 & Galb1-4GIcNAcb1-6(Galb1-4GIcNAcb1-3)GalNAca-Sp8 & 15 & 9 & 61 \\
\hline 285 & Neu5Gca2-6GalNAca-Sp0 & 15 & 16 & 109 \\
\hline 597 & Neu5Aca2-6Galb1-4GlcNAcb1-3Galb1-4GIcNAcb1-3GalNAca-Sp14 & 15 & 9 & 65 \\
\hline 337 & Neu5Aca2-3Galb1-4(Fuca1-3)GlcNAcb1-6(Neu5Aca2-3Galb1-3)GalNAc-Sp14 & 14 & 8 & 52 \\
\hline 174 & GlcNAca1-6Galb1-4GlcNAcb-Sp8 & 14 & 10 & 72 \\
\hline 270 & Neu5Aca2-6Galb1-4GIcNAcb1-3Galb1-4(Fuca1-3)GIcNAcb1-3Galb1-4(Fuca1-3)GIcNAcb-Sp0 & 14 & 12 & 85 \\
\hline 262 & Fuca1-2Galb1-4(6S)Glcb-Sp0 & 14 & 4 & 25 \\
\hline 510 & Galb1-3(6S)GlcNAcb-Sp8 & 14 & 19 & 130 \\
\hline 194 & GlcNAcb1-6Galb1-4GlcNAcb-Sp8 & 14 & 14 & 96 \\
\hline 102 & Gala1-3(Fuca1-2)Galb1-3GIcNAcb-Sp8 & 14 & 3 & 20 \\
\hline
\end{tabular}




\begin{tabular}{|c|c|c|c|c|}
\hline 76 & Fuca1-2Galb1-4GlcNAcb-Sp8 & 14 & 8 & 54 \\
\hline 567 & Galb1-3GIcNAcb1-6(Galb1-3)GalNAc-Sp14 & 14 & 7 & 48 \\
\hline 193 & GIcNAcb1-6GalNAca-Sp14 & 14 & 4 & 28 \\
\hline 67 & Fuca1-2Galb1-3GlcNAcb-Sp0 & 14 & 12 & 82 \\
\hline 74 & Fuca1-2Galb1-4GIcNAcb1-3Galb1-4GIcNAcb1-3Galb1-4GIcNAcb-Sp0 & 14 & 1 & 7 \\
\hline 561 & GlcNAcb1-3Galb1-4GlcNAcb1-6(GlcNAcb1-3Galb1-3)GalNAca-Sp14 & 14 & 10 & 69 \\
\hline 363 & Gala1-3Galb1-4GIcNAcb1-2Mana1-6(Gala1-3Galb1-4GIcNAcb1-2Mana1-3)Manb1-4GIcNAcb1-4GIcNAcb-Sp20 & 14 & 13 & 93 \\
\hline 344 & GlcNAca1-4Galb1-3GalNAc-Sp14 & 14 & 15 & 106 \\
\hline 454 & $\begin{array}{l}\text { GalNAca1-3(Fuca1-2)Galb1-4GIcNAcb1-2Mana1-6(GalNAca1-3(Fuca1-2)Galb1-4GlcNAcb1-2Mana1-3)Manb1- } \\
\text { 4GlcNAcb1-4(Fuca1-6)GlcNAcb-Sp22 }\end{array}$ & 14 & 8 & 54 \\
\hline 431 & GlcNAcb1-2Mana1-6(GlcNAcb1-4)(GlcNAcb1-2Mana1-3)Manb1-4GlcNAcb1-4GlcNAc-Sp21 & 14 & 5 & 38 \\
\hline 420 & $\begin{array}{l}\text { Fuca1-2Galb1-4GIcNAcb1-2Mana1-6(Fuca1-2Galb1-4GIcNAcb1-2Mana1-3)Manb1-4GlcNAcb1-4(Fuca1- } \\
\text { 6)GIcNAcb-Sp22 }\end{array}$ & 14 & 11 & 83 \\
\hline 457 & $\begin{array}{l}\text { GalNAca1-3(Fuca1-2)Galb1-3GIcNAcb1-2Mana1-6(GalNAca1-3(Fuca1-2)Galb1-3GlcNAcb1-2Mana1-3)Manb1- } \\
\text { 4GlcNAcb1-4(Fuca1-6)GlcNAcb-Sp22 }\end{array}$ & 14 & 3 & 22 \\
\hline 78 & Fuca1-2Galb-Sp8 & 14 & 7 & 51 \\
\hline 574 & $\begin{array}{l}\text { GIcNAcb1-3Galb1-4GIcNAcb1-2Mana1-6(GlcNAcb1-3Galb1-4GIcNAcb1-2Mana1-3)Manb1-4GIcNAcb1- } \\
\text { 4(Fuca1-6)GIcNAcb-Sp24 }\end{array}$ & 14 & 9 & 68 \\
\hline 179 & GlcNAcb1-3GalNAca-Sp8 & 14 & 7 & 53 \\
\hline 209 & Mana1-2Mana1-3Mana-Sp9 & 13 & 8 & 57 \\
\hline 326 & $\begin{array}{l}\text { Neu5Aca2-3Galb1-4GlcNAcb1-2Mana1-6(Neu5Aca2-6Galb1-4GlcNAcb1-2Mana1-3)Manb1-4GIcNAcb1- } \\
\text { 4GIcNAcb-Sp12 }\end{array}$ & 13 & 8 & 56 \\
\hline 264 & Neu5Aca2-3Galb1-4GIcb-Sp8 & 13 & 11 & 79 \\
\hline 356 & KDNa2-3Galb1-4(Fuca1-3)GIcNAc-Sp0 & 13 & 11 & 85 \\
\hline 471 & Neu5Aca2-3Galb1-4GIcNAcb1-6(Neu5Aca2-3Galb1-4GIcNAcb1-3)GalNAca-Sp14 & 13 & 3 & 22 \\
\hline 365 & $\begin{array}{l}\text { Fuca1-4(Galb1-3)GIcNAcb1-2Mana1-6(Fuca1-4(Galb1-3)GIcNAcb1-2Mana1-3)Manb1-4GIcNAcb1-4(Fuca1- } \\
\text { 6)GlcNAcb-Sp22 }\end{array}$ & 13 & 4 & 32 \\
\hline 152 & Galb1-4(Fuca1-3)GlcNAcb-Sp8 & 13 & 13 & 96 \\
\hline 10 & Neu5Aca-Sp11 & 13 & 16 & 123 \\
\hline 286 & Neu5Gca2-6Galb1-4GIcNAcb-Sp0 & 13 & 9 & 68 \\
\hline 519 & GalNAca1-3(Fuca1-2)Galb1-4GIcNAcb1-6GalNAc-Sp14 & 13 & 12 & 89 \\
\hline 301 & Neu5Aca2-6Galb1-4GIcNAcb1-2Mana1-6(Galb1-4GIcNAcb1-2Mana1-3)Manb1-4GIcNAcb1-4GIcNAcb-Sp12 & 13 & 6 & 43 \\
\hline 526 & Neu5Aca2-3Galb1-3GIcNAcb1-2Mana-Sp0 & 13 & 10 & 74 \\
\hline 522 & Gala1-3(Fuca1-2)Galb1-4GIcNAcb1-2Mana-Sp0 & 13 & 8 & 62 \\
\hline 62 & Fuca1-2Galb1-3GalNAca-Sp14 & 13 & 17 & 128 \\
\hline 407 & Galb1-3GIcNAca1-6Galb1-4GIcNAcb-Sp0 & 13 & 8 & 63 \\
\hline 268 & Neu5Aca2-6Galb1-4GIcNAcb-Sp0 & 13 & 8 & 61 \\
\hline 184 & GIcNAcb1-3Galb1-4GIcNAcb1-3Galb1-4GIcNAcb-Sp0 & 13 & 8 & 65 \\
\hline 235 & Neu5Aca2-6(Neu5Aca2-3)GalNAca-Sp8 & 13 & 19 & 149 \\
\hline
\end{tabular}




\begin{tabular}{|c|c|c|c|c|}
\hline 110 & Gala1-3GalNAca-Sp8 & 13 & 18 & 145 \\
\hline 216 & Mana1-6(Mana1-3)Mana1-6(Mana1-3)Manb1-4GIcNAcb1-4GIcNAcb-Sp12 & 13 & 3 & 26 \\
\hline 333 & Gala1-4Galb1-4GlcNAcb1-3Galb1-4Glcb-Sp0 & 13 & 8 & 61 \\
\hline 255 & Neu5Aca2-3Galb1-4(Fuca1-3)GIcNAcb-Sp8 & 12 & 8 & 67 \\
\hline 224 & Neu5Aca2-3Galb1-3GalNAca-Sp14 & 12 & 7 & 53 \\
\hline 462 & $\begin{array}{l}\text { Neu5Aca2-3Galb1-4GIcNAcb1-6(Neu5Aca2-3Galb1-4GlcNAcb1-2)Mana1-6(GlcNAcb1-4)(Neu5Aca2-3Galb1- } \\
\text { 4GIcNAcb1-4(Neu5Aca2-3Galb1-4GIcNAcb1-2)Mana1-3)Manb1-4GIcNAcb1-4GlcNAcb-Sp21 }\end{array}$ & 12 & 5 & 42 \\
\hline 327 & $\begin{array}{l}\text { Galb1-4(Fuca1-3)GIcNAcb1-2Mana1-6(Galb1-4(Fuca1-3)GIcNAcb1-2Mana1-3)Manb1-4GIcNAcb1-4GlcNAcb- } \\
\text { Sp20 }\end{array}$ & 12 & 10 & 85 \\
\hline 2 & Glca-Sp8 & 12 & 16 & 130 \\
\hline 426 & $\begin{array}{l}\text { Fuca1-2Galb1-3GIcNAcb1-2Mana1-6(Fuca1-2Galb1-3GIcNAcb1-2Mana1-3)Manb1-4GlcNAcb1-4(Fuca1- } \\
\text { 6)GIcNAcb-Sp22 }\end{array}$ & 12 & 10 & 86 \\
\hline 348 & Neu5Aca2-6Galb1-4GIcNAcb1-2Mana1-3Manb1-4GIcNAcb1-4GIcNAc-Sp12 & 12 & 3 & 26 \\
\hline 61 & Fuca1-2Galb1-3GalNAca-Sp8 & 12 & 15 & 124 \\
\hline 589 & Galb1-4GIcNAcb1-3Galb1-4GIcNAcb1-3GalNAca-Sp14 & 12 & 7 & 59 \\
\hline 32 & (3S)Galb1-4(Fuca1-3)GlcNAc-Sp0 & 12 & 4 & 31 \\
\hline 299 & Neu5Aca2-3Galb1-4(Fuca1-3)GlcNAcb1-6(Galb1-3)GalNAca-Sp14 & 12 & 17 & 146 \\
\hline 266 & Neu5Aca2-6GalNAcb1-4GlcNAcb-Sp0 & 12 & 17 & 147 \\
\hline 554 & Neu5Gca2-8Neu5Aca2-3Galb1-4GlcNAc-Sp0 & 12 & 3 & 29 \\
\hline 396 & $\begin{array}{l}\text { Neu5Aca2-3Galb1-3GlcNAcb1-2Mana1-6(Neu5Aca2-3Galb1-3GlcNAcb1-2Mana1-3)Manb1-4GlcNAcb1- } \\
\text { 4GlcNAc-Sp19 }\end{array}$ & 12 & 8 & 68 \\
\hline 47 & (6S)GlcNAcb-Sp8 & 12 & 9 & 78 \\
\hline 160 & Galb1-4GIcNAcb1-3GalNAc-Sp14 & 12 & 2 & 18 \\
\hline 258 & Neu5Aca2-3Galb1-4GIcNAcb1-3Galb1-4GIcNAcb1-3Galb1-4GIcNAcb-Sp0 & 12 & 8 & 65 \\
\hline 353 & Galb1-4GIcNAcb1-2Mana1-6(Galb1-4GIcNAcb1-2Mana1-3)Manb1-4GlcNAcb1-4(Fuca1-6)GIcNAcb-Sp22 & 12 & 6 & 51 \\
\hline 506 & GalNAca1-3(Fuca1-2)Galb1-3GIcNAcb1-6GalNAca-Sp14 & 12 & 11 & 92 \\
\hline 402 & GaINAca1-3GalNAcb1-3Gala1-4Galb1-4GIcNAcb-Sp0 & 12 & 5 & 46 \\
\hline 376 & Neu5Aca2-3Galb1-4GIcNAcb1-3GalNAc-Sp14 & 12 & 7 & 56 \\
\hline 20 & Galb1-4GIcNAcb1-6(Galb1-4GlcNAcb1-3)GalNAc-Sp14 & 12 & 8 & 73 \\
\hline 133 & GlcNAcb1-6(Galb1-3)GalNAca-Sp8 & 12 & 2 & 15 \\
\hline 66 & Fuca1-2Galb1-3GIcNAcb1-3Galb1-4GIcb-Sp10 & 11 & 10 & 84 \\
\hline 378 & Neu5Aca2-3Galb1-4(Fuca1-3)GIcNAcb1-3GalNAca-Sp14 & 11 & 6 & 54 \\
\hline 492 & (3S)Galb1-3(Fuca1-4)GIcNAcb-Sp0 & 11 & 9 & 82 \\
\hline 418 & GalNAca1-3(Fuca1-2)Galb1-4(Fuca1-3)GlcNAcb1-3GalNAc-Sp14 & 11 & 13 & 111 \\
\hline 222 & Fuca1-2(6S)Galb1-4(6S)Glcb-Sp0 & 11 & 7 & 62 \\
\hline 70 & Fuca1-2Galb1-4(Fuca1-3)GlcNAcb1-3Galb1-4(Fuca1-3)GlcNAcb1-3Galb1-4(Fuca1-3)GlcNAcb-Sp0 & 11 & 6 & 53 \\
\hline 474 & $\begin{array}{l}\text { Neu5Aca2-3Galb1-3GlcNAcb1-6(Neu5Aca2-3Galb1-3GlcNAcb1-2)Mana1-6(Neu5Aca2-3Galb1-3GlcNAcb1- } \\
\text { 2Mana1-3)Manb1-4GIcNAcb1-4GlcNAcb-Sp19 }\end{array}$ & 11 & 3 & 32 \\
\hline 555 & Neu5Gca2-8Neu5Gca2-3Galb1-4GlcNAcb1-3Galb1-4GlcNAc-Sp0 & 11 & 5 & 44 \\
\hline 51 & Mana1-6(Mana1-3)Manb1-4GIcNAcb1-4GIcNAcb-Sp13 & 11 & 13 & 117 \\
\hline
\end{tabular}




\begin{tabular}{|c|c|c|c|c|}
\hline 390 & Fuca1-2Galb1-3GalNAca1-3(Fuca1-2)Galb1-4GIcNAcb-Sp0 & 11 & 8 & 69 \\
\hline 588 & $\begin{array}{l}\text { Galb1-4GIcNAcb1-3Galb1-4GlcNAcb1-3Galb1-4GlcNAcb1-3Galb1-4GlcNAcb1-3Galb1-4GlcNAcb1-6(Galb1- } \\
\text { 4GlcNAcb1-3Galb1-4GlcNAcb1-3Galb1-4GlcNAcb1-3Galb1-4GlcNAcb1-3Galb1-4GlcNAb1-2)Mana1-6(Galb1- } \\
\text { 4GlcNAcb1-3Galb1-4GlcNAcb1-3Galb1-4GlcNAcb1-3Galb1-4GlcNAcb1-3Galb1-4GlcNAcb1-2Mana1-3)Manb1- } \\
\text { 4GlcNAcb1-4(Fuca1-6)GlcNAcb-Sp24 }\end{array}$ & 11 & 8 & 72 \\
\hline 508 & $\begin{array}{l}\text { Galb1-4GIcNAcb1-6(Galb1-4GIcNAcb1-2)Mana1-6(GlcNAcb1-4)Galb1-4GIcNAcb1-4(Gal b1-4GlcNAcb1- } \\
\text { 2)Mana1-3)Manb1-4GlcNAcb1-4(Fuca1-6)GlcNAc-Sp21 }\end{array}$ & 11 & 12 & 107 \\
\hline 142 & Galb1-3GalNAcb-Sp8 & 11 & 11 & 104 \\
\hline 476 & Galb1-3GlcNAcb1-2Mana1-6(GlcNAcb1-4)(Galb1-3GlcNAcb1-2Mana1-3)Manb1-4GlcNAcb1-4GlcNAcb-Sp21 & 11 & 10 & 91 \\
\hline 245 & Neu5Aca2-3Galb-Sp8 & 11 & 7 & 60 \\
\hline 598 & GlcNAcb1-3Galb1-4GIcNAcb1-3Galb1-4GIcNAcb1-3GalNAca-Sp14 & 11 & 6 & 58 \\
\hline 441 & Neu5Aca2-3Galb1-4GIcNAcb1-3Galb-Sp8 & 11 & 15 & 136 \\
\hline 265 & Neu5Aca2-6GalNAca-Sp8 & 11 & 17 & 165 \\
\hline 101 & Gala1-3(Fuca1-2)Galb1-3GIcNAcb-Sp0 & 10 & 5 & 46 \\
\hline 606 & $\begin{array}{l}\text { Neu5Aca2-6Galb1-4GlcNAcb1-3Galb1-4GlcNAcb1-3Galb1-4GIcNAcb1-2Mana1-6(Neu5Aca2-6Galb1- } \\
\text { 4GIcNAcb1-3Galb1-4GIcNAcb1-3Galb1-4GlcNAcb1-2Mana1-3)Manb1-4GIcNAcb1-4GlcNAcb-Sp12 }\end{array}$ & 10 & 7 & 67 \\
\hline 173 & GlcNAca1-3Galb1-4GlcNAcb-Sp8 & 10 & 19 & 183 \\
\hline 287 & Neu5Gca-Sp8 & 10 & 8 & 78 \\
\hline 275 & Neu5Aca2-8Neu5Aca-Sp8 & 10 & 9 & 93 \\
\hline 249 & Neu5Aca2-3Galb1-3GlcNAcb-Sp0 & 10 & 2 & 20 \\
\hline 269 & Neu5Aca2-6Galb1-4GlcNAcb-Sp8 & 10 & 14 & 136 \\
\hline 36 & (3S)Galb1-4GlcNAcb-Sp0 & 10 & 12 & 115 \\
\hline 438 & $\begin{array}{l}\text { Galb1-4GIcNAcb1-6(Galb1-4GIcNAcb1-2)Mana1-6(GlcNAcb1-4)(Galb1-4GlcNAcb1-4(Galb1-4GlcNAcb1- } \\
\text { 2)Mana1-3)Manb1-4GlcNAcb1-4GlcNAc-Sp21 }\end{array}$ & 10 & 6 & 63 \\
\hline 410 & Galb1-4GlcNAcb1-6(Neu5Aca2-6Galb1-3GlcNAcb1-3)Galb1-4Glc-Sp21 & 10 & 3 & 32 \\
\hline 46 & Neu5Aca2-3(6S)Galb1-4GIcNAcb-Sp8 & 10 & 7 & 72 \\
\hline 440 & Galb1-6Galb-Sp10 & 10 & 2 & 23 \\
\hline 237 & Neu5Aca2-3GalNAcb1-4GIcNAcb-Sp0 & 10 & 8 & 81 \\
\hline 106 & Gala1-3(Fuca1-2)Galb1-4GIcb-Sp0 & 10 & 6 & 60 \\
\hline 352 & GlcNAcb1-2Mana1-6(GlcNAcb1-2Mana1-3)Manb1-4GlcNAcb1-4(Fuca1-6)GIcNAcb-Sp22 & 10 & 6 & 58 \\
\hline 600 & Neu5Aca2-3Galb1-4GIcNAcb1-3Galb1-4GlcNAcb1-6(Galb1-3)GalNAca-Sp14 & 10 & 9 & 93 \\
\hline 108 & Gala1-3(Fuca1-2)Galb-Sp18 & 10 & 7 & 67 \\
\hline 189 & GlcNAcb1-4GlcNAcb1-4GlcNAcb1-4GlcNAcb1-4GlcNAcb1-4GlcNAcb1-Sp8 & 10 & 14 & 141 \\
\hline 556 & Neu5Gca2-8Neu5Gca2-6Galb1-4GIcNAc-Sp0 & 10 & 6 & 64 \\
\hline 41 & (6P)Mana-Sp8 & 10 & 13 & 129 \\
\hline 252 & Neu5Aca2-3Galb1-4(Fuca1-3)(6S)GIcNAcb-Sp8 & 10 & 11 & 112 \\
\hline 347 & Neu5Aca2-6Galb1-4GIcNAcb1-2Mana1-6Manb1-4GlcNAcb1-4GIcNAc-Sp12 & 10 & 12 & 123 \\
\hline 191 & GlcNAcb1-4GlcNAcb1-4GlcNAcb-Sp8 & 10 & 6 & 65 \\
\hline 283 & Neu5Gca2-3Galb1-4GlcNAcb-Sp0 & 10 & 6 & 63 \\
\hline 157 & Galb1-4GalNAca1-3(Fuca1-2)Galb1-4GlcNAcb-Sp8 & 10 & 9 & 95 \\
\hline
\end{tabular}




\begin{tabular}{|c|c|c|c|c|}
\hline 84 & (3S)Galb1-4(Fuca1-3)Glcb-Sp0 & 10 & 11 & 111 \\
\hline 398 & Galb1-4GIcNAcb1-2Mana1-6(GIcNAcb1-2Mana1-3)Manb1-4GIcNAcb1-4GIcNAc-Sp12 & 9 & 5 & 51 \\
\hline 465 & $\begin{array}{l}\text { Neu5Aca2-6Galb1-4GIcNAcb1-6(Neu5Aca2-6Galb1-4GIcNAcb1-2)Mana1-6(GlcNAcb1-4)(Neu5Aca2-6Galb1- } \\
\text { 4GIcNAcb1-2Mana1-3)Manb1-4GIcNAcb1-4GIcNAcb-Sp21 }\end{array}$ & 9 & 7 & 71 \\
\hline 318 & $\begin{array}{l}\text { Neu5Aca2-6Galb1-4GIcNAcb1-2Mana1-6(Neu5Aca2-3Galb1-4GlcNAcb1-2Mana1-3)Manb1-4GIcNAcb1- } \\
\text { 4GIcNAcb-Sp12 }\end{array}$ & 9 & 4 & 44 \\
\hline 499 & Fuca1-2Galb1-4GIcNAcb1-2Mana-Sp0 & 9 & 10 & 113 \\
\hline 175 & GlcNAcb1-2Galb1-3GalNAca-Sp8 & 9 & 7 & 77 \\
\hline 201 & GlcAa-Sp8 & 9 & 9 & 100 \\
\hline 537 & Gala1-3(Fuca1-2)Galb1-3GalNAcb1-3Gala1-4Galb1-4Glc-Sp21 & 9 & 10 & 116 \\
\hline 449 & Fuca1-2Galb1-4GIcNAcb1-6(Fuca1-2Galb1-4GIcNAcb1-3)GalNAc-Sp14 & 9 & 5 & 55 \\
\hline 49 & Neu5,9Ac2a2-6Galb1-4GlcNAcb-Sp8 & 9 & 3 & 37 \\
\hline 532 & $\begin{array}{l}\text { Galb1-4GlcNAcb1-2 Mana1-6(Galb1-4GlcNAcb1-4)(Galb1-4GlcNAcb1-2Mana1-3)Manb1-4GIcNAcb1-4(Fuca1- } \\
\text { 6)GlcNAc-Sp21 }\end{array}$ & 9 & 14 & 155 \\
\hline 345 & Neu5Aca2-6Galb1-4GIcNAcb1-2Mana1-6(Mana1-3)Manb1-4GIcNAcb1-4GIcNAc-Sp12 & 9 & 9 & 101 \\
\hline 300 & Galb1-3Galb1-4GIcNAcb-Sp8 & 9 & 4 & 44 \\
\hline 177 & GlcNAcb1-6(GlcNAcb1-3)GalNAca-Sp14 & 9 & 10 & 121 \\
\hline 379 & GalNAcb1-4GIcNAcb1-2Mana1-6(GalNAcb1-4GIcNAcb1-2Mana1-3)Manb1-4GIcNAcb1-4GIcNAc-Sp12 & 9 & 5 & 54 \\
\hline 339 & GlcNAca1-4Galb1-4GlcNAcb-Sp0 & 9 & 4 & 45 \\
\hline 335 & GalNAca1-3(Fuca1-2)Galb1-4GIcNAcb1-3Galb1-4GIcNAcb-Sp0 & 9 & 8 & 95 \\
\hline 3 & Mana-Sp8 & 8 & 6 & 71 \\
\hline 459 & $\begin{array}{l}\text { Neu5Aca2-3Galb1-4GIcNAcb1-2Mana1-6(GIcNAcb1-4)(Neu5Aca2-3Galb1-4GIcNAcb1-2Mana1-3)Manb1- } \\
\text { 4GIcNAcb1-4GIcNAcb-Sp21 }\end{array}$ & 8 & 6 & 74 \\
\hline 80 & Fuca1-4GIcNAcb-Sp8 & 8 & 7 & 86 \\
\hline 380 & Galb1-3GalNAca1-3(Fuca1-2)Galb1-4GIc-Sp0 & 8 & 7 & 89 \\
\hline 267 & Neu5Aca2-6Galb1-4(6S)GlcNAcb-Sp8 & 8 & 3 & 42 \\
\hline 304 & Galb1-4GIcNAca1-6Galb1-4GIcNAcb-Sp0 & 8 & 10 & 123 \\
\hline 203 & GlcAb1-3Galb-Sp8 & 8 & 9 & 112 \\
\hline 305 & Galb1-4GIcNAcb1-6Galb1-4GIcNAcb-Sp0 & 8 & 4 & 46 \\
\hline 317 & Neu5Aca2-3Galb1-4GIcNAcb1-6(Neu5Aca2-3Galb1-3)GalNAca-Sp14 & 8 & 7 & 88 \\
\hline 334 & GalNAcb1-3Gala1-4Galb1-4GIcNAcb1-3Galb1-4Glcb-Sp0 & 8 & 6 & 70 \\
\hline 148 & Galb1-3GIcNAcb1-3Galb1-4Glcb-Sp10 & 8 & 10 & 116 \\
\hline 603 & $\begin{array}{l}\text { Neu5Aca2-3Galb1-4GlcNAcb1-3Galb1-4GIcNAcb1-2Mana1-6(Neu5Aca2-3Galb1-4GlcNAcb1-3Galb1- } \\
\text { 4GIcNAcb1-2Mana1-3)Manb1-4GlcNAcb1-4GlcNAcb-Sp12 }\end{array}$ & 8 & 11 & 136 \\
\hline 294 & Neu5Aca2-3Galb1-3GlcNAcb1-3Galb1-3GlcNAcb-Sp0 & 8 & 12 & 157 \\
\hline 29 & (3S)Galb1-3GalNAca-Sp8 & 8 & 4 & 47 \\
\hline
\end{tabular}




\begin{tabular}{|c|c|c|c|c|}
\hline 178 & GlcNAcb1-6(GlcNAcb1-3)Galb1-4GIcNAcb-Sp8 & 8 & 4 & 44 \\
\hline 401 & Galb1-4(Fuca1-3)GıcNAcb1-3GalNAca-Sp14 & 8 & 9 & 110 \\
\hline 477 & Neu5Aca2-6Galb1-4GIcNAcb1-6(Galb1-3GlcNAcb1-3)Galb1-4Glcb-Sp21 & 8 & 11 & 140 \\
\hline 539 & $\begin{array}{l}\text { GlcNAcb1-3Galb1-4GlcNAcb1-2Mana1-6(GlcNAcb1-3Galb1-4GlcNAcb1-2Mana1-3)Manb1-4GlcNAcb1- } \\
\text { 4GlcNAcb-Sp12 }\end{array}$ & 8 & 5 & 70 \\
\hline 43 & (6S)Galb1-4Glcb-Sp8 & 8 & 7 & 95 \\
\hline 188 & GlcNAcb1-4Galb1-4GlcNAcb-Sp8 & 8 & 11 & 143 \\
\hline 303 & GlcNAcb1-6(Galb1-4GIcNAcb1-3)Galb1-4GIcNAc-Sp0 & 8 & 6 & 82 \\
\hline 12 & Galb-Sp8 & 8 & 10 & 134 \\
\hline 460 & $\begin{array}{l}\text { Neu5Aca2-3Galb1-4GlcNAcb1-4Mana1-6(GlcNAcb1-4)(Neu5Aca2-3Galb1-4GlcNAcb1-4(Neu5Aca2-3Galb1- } \\
\text { 4GlcNAcb1-2)Mana1-3)Manb1-4GlcNAcb1-4GlcNAcb-Sp21 }\end{array}$ & 8 & 7 & 92 \\
\hline 272 & Neu5Aca2-6Galb1-4Glcb-Sp0 & 8 & 10 & 135 \\
\hline 150 & Galb1-3GIcNAcb-Sp8 & 8 & 15 & 201 \\
\hline 244 & Neu5Aca2-6(Neu5Aca2-3Galb1-3)GalNAca-Sp14 & 8 & 8 & 103 \\
\hline 211 & Mana1-2Mana1-6(Mana1-3)Mana1-6(Mana1-2Mana1-2Mana1-3)Manb1-4GIcNAcb1-4GIcNAcb-Sp12 & 8 & 13 & 168 \\
\hline 186 & GlcNAcb1-4-MDPLys & 7 & 5 & 71 \\
\hline 430 & Fuca1-3GlcNAcb1-6(Galb1-4GlcNAcb1-3)Galb1-4Glc-Sp21 & 7 & 4 & 52 \\
\hline 330 & Neu5Aca2-6Galb1-4GIcNAcb1-3Galb1-3GIcNAcb-Sp0 & 7 & 3 & 36 \\
\hline 127 & Galb1-3GIcNAcb1-3Galb1-4(Fuca1-3)GIcNAcb-Sp0 & 7 & 3 & 44 \\
\hline 250 & Neu5Aca2-3Galb1-3GlcNAcb-Sp8 & 7 & 6 & 87 \\
\hline 435 & Galb1-4GIcNAcb1-2Mana1-6(GlcNAcb1-4)(Galb1-4GIcNAcb1-2Mana1-3)Manb1-4GlcNAcb1-4GIcNAc-Sp21 & 7 & 4 & 62 \\
\hline 140 & Galb1-3GalNAca-Sp14 & 7 & 10 & 137 \\
\hline 427 & $\begin{array}{l}\text { Gala1-3(Fuca1-2)Galb1-4GlcNAcb1-2Mana1-6(Gala1-3(Fuca1-2)Galb1-4GlcNAcb1-2Mana1-3)Manb1- } \\
\text { 4GlcNAcb1-4(Fuca1-6)GlcNAcb-Sp22 }\end{array}$ & 7 & 4 & 58 \\
\hline 349 & Galb1-4GIcNAcb1-2Mana1-3Manb1-4GIcNAcb1-4GIcNAc-Sp12 & 7 & 12 & 163 \\
\hline 315 & Mana1-2Mana1-6(Mana1-3)Mana1-6(Mana1-2Mana1-2Mana1-3)Mana-Sp9 & 7 & 6 & 79 \\
\hline 115 & Gala1-3Galb1-4GIcNAcb-Sp8 & 7 & 16 & 233 \\
\hline 397 & GIcNAcb1-2Mana1-6(Galb1-4GIcNAcb1-2Mana1-3)Manb1-4GIcNAcb1-4GIcNAc-Sp12 & 7 & 10 & 145 \\
\hline 487 & Neu5Aca2-6Galb1-4GIcNAcb1-6(Fuca1-2Galb1-4(Fuca1-3)GlcNAcb1-3)Galb1-4GIc-Sp21 & 7 & 17 & 244 \\
\hline 351 & Mana1-6(Galb1-4GlcNAcb1-2Mana1-3)Manb1-4GlcNAcb1-4GlcNAcb-Sp12 & 7 & 4 & 55 \\
\hline 151 & Galb1-4(Fuca1-3)GlcNAcb-Sp0 & 7 & 12 & 179 \\
\hline 323 & Neu5Gcb2-6Galb1-4GIcNAc-Sp8 & 7 & 11 & 160 \\
\hline 368 & Galb1-4(Fuca1-3)GIcNAcb1-6(Fuca1-2Galb1-4GIcNAcb1-3)Galb1-4GIc-Sp21 & 7 & 4 & 64 \\
\hline 442 & GalNAcb1-6GalNAcb-Sp8 & 7 & 5 & 77 \\
\hline 504 & (3S)GalNAcb1-4(Fuca1-3)GlcNAcb-Sp8 & 7 & 7 & 106 \\
\hline 502 & Neu5Aca2-6GalNAcb1-4(6S)GlcNAcb-Sp8 & 7 & 7 & 106 \\
\hline 197 & Glca1-6Glca1-6Glcb-Sp8 & 7 & 6 & 97 \\
\hline 128 & Galb1-3(Fuca1-4)GIcNAc-Sp0 & 7 & 5 & 70 \\
\hline
\end{tabular}




\begin{tabular}{|c|c|c|c|c|}
\hline 130 & Fuca1-4(Galb1-3)GlcNAcb-Sp8 & 7 & 8 & 125 \\
\hline 271 & Neu5Aca2-6Galb1-4GIcNAcb1-3Galb1-4GIcNAcb-Sp0 & 7 & 12 & 176 \\
\hline 381 & Galb1-3GalNAca1-3(Fuca1-2)Galb1-4GlcNAc-Sp0 & 6 & 5 & 83 \\
\hline 289 & Galb1-3GlcNAcb1-3Galb1-3GlcNAcb-Sp0 & 6 & 6 & 92 \\
\hline 163 & Galb1-4GIcNAcb1-3Galb1-4GIcNAcb-Sp0 & 6 & 6 & 93 \\
\hline 243 & Neu5Aca2-6(Neu5Aca2-3Galb1-3)GalNAca-Sp8 & 6 & 10 & 159 \\
\hline 30 & (3S)Galb1-3GlcNAcb-Sp0 & 6 & 1 & 16 \\
\hline 528 & GalNAcb1-4GlcNAcb1-2Mana-Sp0 & 6 & 13 & 211 \\
\hline 15 & GalNAcb-Sp8 & 6 & 8 & 139 \\
\hline 164 & Galb1-4GlcNAcb1-3Galb1-4Glcb-Sp0 & 6 & 6 & 99 \\
\hline 458 & $\begin{array}{l}\text { Galb1-4GIcNAcb1-6(Galb1-4GlcNAcb1-2)Mana1-6(Galb1-4GIcNAcb1-2Mana1-3)Manb1-4GIcNAcb1- } \\
\text { 4GIcNAcb-Sp19 }\end{array}$ & 6 & 6 & 107 \\
\hline 129 & Galb1-3(Fuca1-4)GIcNAc-Sp8 & 6 & 6 & 106 \\
\hline 594 & GlcNAcb1-3Galb1-4GIcNAcb1-6(Galb1-3)GalNAca-Sp14 & 6 & 16 & 274 \\
\hline 79 & Fuca1-3GIcNAcb-Sp8 & 6 & 5 & 85 \\
\hline 433 & GlcNAcb1-6(GlcNAcb1-2)Mana1-6(GlcNAcb1-4)(GlcNAcb1-2Mana1-3)Manb1-4GIcNAcb1-4GIcNAc-Sp21 & 6 & 5 & 95 \\
\hline 125 & Galb1-2Galb-Sp8 & 6 & 10 & 187 \\
\hline 143 & Galb1-3GalNAcb1-3Gala1-4Galb1-4Glcb-Sp0 & 5 & 3 & 64 \\
\hline 521 & Gala1-3Galb1-4GIcNAcb1-2Mana-Sp0 & 5 & 6 & 106 \\
\hline 260 & Neu5Aca2-3Galb1-4GlcNAcb-Sp8 & 5 & 7 & 133 \\
\hline 346 & Mana1-6(Neu5Aca2-6Galb1-4GIcNAcb1-2Mana1-3)Manb1-4GIcNAcb1-4GIcNAc-Sp12 & 5 & 2 & 35 \\
\hline 82 & GalNAca1-3(Fuca1-2)Galb1-3GIcNAcb-Sp0 & 5 & 4 & 80 \\
\hline 291 & Galb1-4(Fuca1-3)(6S)Glcb-Sp0 & 5 & 7 & 142 \\
\hline 217 & Manb1-4GlcNAcb-Sp0 & 5 & 7 & 154 \\
\hline 341 & GlcNAca1-4Galb1-4GlcNAcb1-3Galb1-4Glcb-Sp0 & 5 & 8 & 161 \\
\hline 176 & GlcNAcb1-6(GlcNAcb1-3)GalNAca-Sp8 & 5 & 5 & 105 \\
\hline 199 & Glcb1-6Glcb-Sp8 & 5 & 3 & 58 \\
\hline 113 & Gala1-3Galb1-4(Fuca1-3)GlcNAcb-Sp8 & 5 & 5 & 100 \\
\hline 155 & Galb1-4(6S)Glcb-Sp0 & 5 & 6 & 139 \\
\hline 367 & Neu5Aca2-6GIcNAcb1-4GIcNAcb1-4GIcNAc-Sp21 & 4 & 14 & 306 \\
\hline 374 & $\begin{array}{l}\text { Gala1-3(Fuca1-2)Galb1-3GlcNAcb1-2Mana1-6(Gala1-3(Fuca1-2)Galb1-3GlcNAcb1-2Mana1-3)Manb1- } \\
\text { 4GlcNAcb1-4GlcNAcb-Sp20 }\end{array}$ & 4 & 5 & 110 \\
\hline 196 & Glca1-4Glca-Sp8 & 4 & 21 & 476 \\
\hline 469 & Glca1-6Glca1-6Glca1-6Glcb-Sp10 & 4 & 8 & 186 \\
\hline 100 & Gala1-2Galb-Sp8 & 4 & 13 & 308 \\
\hline 60 & Fuca1-2Galb1-3(Fuca1-4)GIcNAcb-Sp8 & 4 & 3 & 67 \\
\hline 210 & Mana1-6(Mana1-2Mana1-3)Mana1-6(Mana1-2Mana1-3)Manb1-4GlcNAcb1-4GIcNAcb-Sp12 & 4 & 19 & 481 \\
\hline 523 & GalNAca1-3(Fuca1-2)Galb1-4 GIcNAcb1-2Mana-Sp0 & 4 & 7 & 191 \\
\hline 296 & 4S(3S)Galb1-4GIcNAcb-Sp0 & 4 & 1 & 29 \\
\hline
\end{tabular}




\begin{tabular}{|c|c|c|c|c|}
\hline 329 & Neu5,9Ac2a2-3Galb1-3GIcNAcb-Sp0 & 4 & 8 & 214 \\
\hline 228 & GalNAcb1-4(Neu5Aca2-8Neu5Aca2-3)Galb1-4Glcb-Sp0 & 4 & 5 & 124 \\
\hline 281 & Neu5Gca2-3Galb1-3GlcNAcb-Sp0 & 4 & 3 & 69 \\
\hline 202 & GlcAb-Sp8 & 4 & 8 & 223 \\
\hline 497 & Fuca1-2(6S)Galb1-3GIcNAcb-Sp0 & 4 & 13 & 346 \\
\hline 295 & Neu5Aca2-3Galb1-4GIcNAcb1-3Galb1-3GIcNAcb-Sp0 & 4 & 6 & 171 \\
\hline 75 & Fuca1-2Galb1-4GlcNAcb-Sp0 & 4 & 6 & 168 \\
\hline 145 & Galb1-3GalNAcb1-4Galb1-4Glcb-Sp8 & 3 & 13 & 373 \\
\hline 558 & $\begin{array}{l}\text { GlcNAcb1-3Galb1-4GlcNAcb1-6(GlcNAcb1-3Galb1-4GlcNAcb1-2)Mana1-6(GlcNAcb1-3Galb1-4GlcNAcb1- } \\
\text { 2Man a1-3)Manb1-4GlcNAcb1-4GlcNAc-Sp24 }\end{array}$ & 3 & 9 & 276 \\
\hline 416 & Fuca1-2Galb1-4(Fuca1-3)GIcNAcb1-3GalNAca-Sp14 & 3 & 7 & 218 \\
\hline 298 & (6P)Glcb-Sp10 & 3 & 3 & 106 \\
\hline 169 & Galb1-4GlcNAcb-Sp8 & 3 & 8 & 311 \\
\hline 319 & Galb1-4GIcNAcb1-2Mana1-6(Neu5Aca2-6Galb1-4GIcNAcb1-2Mana1-3)Manb1-4GIcNAcb1-4GIcNAcb-Sp12 & 3 & 4 & 137 \\
\hline 206 & KDNa2-3Galb1-4GIcNAcb-Sp0 & 2 & 4 & 166 \\
\hline 187 & GlcNAcb1-6(GlcNAcb1-4)GalNAca-Sp8 & 2 & 3 & 118 \\
\hline 592 & Neu5Aca2-3Galb1-4GIcNAcb1-3Galb1-4GIcNAcb1-3GalNAca-Sp14 & 2 & 13 & 598 \\
\hline 168 & Galb1-4GIcNAcb-Sp0 & 2 & 11 & 529 \\
\hline 479 & Neu5Aca2-3Galb1-4GIcNAcb1-6GalNAca-Sp14 & 2 & 6 & 276 \\
\hline 212 & Mana1-2Mana1-6(Mana1-2Mana1-3)Mana1-6(Mana1-2Mana1-2Mana1-3)Manb1-4GIcNAcb1-4GIcNAcb-Sp12 & 2 & 7 & 388 \\
\hline 257 & Neu5Aca2-3Galb1-4(Fuca1-3)GlcNAcb1-3Galb1-4GlcNAcb-Sp8 & 2 & 3 & 184 \\
\hline 325 & $\begin{array}{l}\text { Neu5Aca2-3Galb1-4GIcNAcb1-2Mana1-6(Neu5Aca2-3Galb1-4GIcNAcb1-2Mana1-3)Manb1-4GIcNAcb1- } \\
\text { 4GIcNAcb-Sp12 }\end{array}$ & 2 & 14 & 800 \\
\hline 16 & GlcNAcb-Sp0 & 2 & 2 & 127 \\
\hline 213 & Mana1-6(Mana1-3)Mana-Sp9 & 2 & 2 & 140 \\
\hline 73 & Fuca1-2Galb1-4GIcNAcb1-3Galb1-4GIcNAcb-Sp0 & 2 & 3 & 223 \\
\hline 309 & GlcNAcb1-3Man-Sp10 & 1 & 3 & 319 \\
\hline 21 & GlcNAcb1-6(GlcNAcb1-4)(GlcNAcb1-3)GlcNAc-Sp8 & 1 & 18 & 1993 \\
\hline 195 & Glca1-4Glcb-Sp8 & 1 & 10 & 1239 \\
\hline 95 & GalNAcb1-3(Fuca1-2)Galb-Sp8 & 1 & 3 & 420 \\
\hline 40 & (4S)Galb1-4GlcNAcb-Sp8 & 1 & 7 & 1052 \\
\hline 463 & $\begin{array}{l}\text { Neu5Aca2-6Galb1-4GIcNAcb1-2Mana1-6(GIcNAcb1-4)(Neu5Aca2-6Galb1-4GlcNAcb1-2Mana1-3)Manb1- } \\
\text { 4GIcNAcb1-4GIcNAcb-Sp21 }\end{array}$ & 1 & 10 & 1900 \\
\hline
\end{tabular}




\begin{tabular}{|c|c|c|c|c|}
\hline 439 & Galb1-4Galb-Sp10 & 0 & 7 & 2695 \\
\hline 94 & GalNAcb1-3GalNAca-Sp8 & 0 & 9 & 3508 \\
\hline 321 & Neu5Aca2-8Neu5Acb-Sp17 & 0 & 7 & 3119 \\
\hline 246 & Neu5Aca2-3Galb1-3GalNAcb1-3Gala1-4Galb1-4GIcb-Sp0 & 0 & 12 & 5377 \\
\hline 4 & GalNAca-Sp8 & 0 & 1 & 1155 \\
\hline 414 & GalNAca1-3(Fuca1-2)Galb1-4GIcNAcb1-3GalNAca-Sp14 & 0 & 5 & 8225 \\
\hline 119 & Gala1-4(Fuca1-2)Galb1-4GIcNAcb-Sp8 & 0 & 20 & 239616 \\
\hline 28 & (3S)Galb1-3(Fuca1-4)GlcNAcb-Sp8 & 0 & 6 & -12592 \\
\hline 491 & Neu5Aca2-3Galb1-3GIcNAcb1-6GalNAca-Sp14 & 0 & 26 & -28973 \\
\hline 50 & Mana1-6(Mana1-3)Manb1-4GlcNAcb1-4GlcNAcb-Sp12 & 0 & 11 & -6325 \\
\hline 220 & Fuca1-2(6S)Galb1-4GIcNAcb-Sp0 & 0 & 11 & -2796 \\
\hline 214 & Mana1-2Mana1-2Mana1-6(Mana1-3)Mana-Sp9 & 0 & 15 & -3196 \\
\hline 372 & $\begin{array}{l}\text { Gala1-3Galb1-4(Fuca1-3)GIcNAcb1-2Mana1-6(Gala1-3Galb1-4(Fuca1-3)GIcNAcb1-2Mana1-3)Manb1- } \\
\text { 4GlcNAcb1-4GIcNAcb-Sp20 }\end{array}$ & 0 & 16 & -3215 \\
\hline 8 & Rhaa-Sp8 & -1 & 11 & -1825 \\
\hline 65 & Fuca1-2Galb1-3GIcNAcb1-3Galb1-4GIcb-Sp8 & -1 & 13 & -1706 \\
\hline 608 & $\begin{array}{l}\text { Neu5Aca2-6Galb1-4GIcNAcb1-3Galb1-4GIcNAcb1-2Mana1-6(Neu5Aca2-6Galb1-4GIcNAcb1-3Galb1- } \\
\text { 4GlcNAcb1-2Mana1-3)Manb1-4GIcNAcb1-4GlcNAcb-Sp12 }\end{array}$ & -1 & 4 & -452 \\
\hline 221 & Fuca1-2Galb1-4(6S)GIcNAcb-Sp8 & -1 & 4 & -369 \\
\hline 466 & $\begin{array}{l}\text { Neu5Aca2-6Galb1-4GIcNAcb1-6(Neu5Aca2-6Galb1-4GlcNAcb1-2)Mana1-6(GlcNAcb1-4)(Neu5Aca2-6Galb1- } \\
\text { 4GlcNAcb1-4(Neu5Aca2-6Galb1-4GIcNAcb1-2)Mana1-3)Manb1-4GlcNAcb1-4GlcNAcb-Sp21 }\end{array}$ & -1 & 6 & -509 \\
\hline 399 & Neu5Aca2-3Galb1-3GIcNAcb1-3GalNAca-Sp14 & -1 & 8 & -596 \\
\hline 481 & Neu5Aca2-6Galb1-4 GlcNAcb1-6(Neu5Aca2-6Galb1-4GlcNAcb1-3)GalNAca-Sp14 & -1 & 8 & -618 \\
\hline 232 & GalNAcb1-4(Neu5Aca2-3)Galb1-4GIcNAcb-Sp8 & -2 & 7 & -459 \\
\hline 225 & GalNAcb1-4(Neu5Aca2-8Neu5Aca2-8Neu5Aca2-8Neu5Aca2-3)Galb1-4Glcb-Sp0 & -2 & 6 & -274 \\
\hline 170 & Galb1-4GlcNAcb-Sp23 & -2 & 11 & -450 \\
\hline 198 & Glcb1-4Glcb-Sp8 & -2 & 13 & -548 \\
\hline 123 & Gala1-4GlcNAcb-Sp8 & -3 & 16 & -541 \\
\hline 557 & Neu5Aca2-8Neu5Aca2-3Galb1-4GIcNAc-Sp0 & -4 & 15 & -407 \\
\hline
\end{tabular}




\begin{tabular}{|c|c|c|c|c|}
\hline 37 & (3S)Galb1-4GlcNAcb-Sp8 & -5 & 7 & -136 \\
\hline 320 & GlcNAcb1-2Mana1-6(Neu5Aca2-6Galb1-4GIcNAcb1-2Mana1-3)Manb1-4GIcNAcb1-4GIcNAcb-Sp12 & -7 & 9 & -131 \\
\hline 278 & Neu5Acb2-6GalNAca-Sp8 & -7 & 10 & -151 \\
\hline 406 & Galb1-3GlcNAcb1-6Galb1-4GlcNAcb-Sp0 & -7 & 17 & -239 \\
\hline 86 & GalNAca1-3(Fuca1-2)Galb1-4GIcNAcb-Sp8 & -8 & 5 & -60 \\
\hline 590 & Galb1-4GIcNAcb1-3Galb1-4GIcNAcb1-6(Galb1-3)GalNAca-Sp14 & -9 & 17 & -184 \\
\hline 117 & Gala1-3Galb1-4Glc-Sp10 & -10 & 5 & -50 \\
\hline 147 & Galb1-3GIcNAcb1-3Galb1-4GIcNAcb-Sp0 & -18 & 6 & -34 \\
\hline 172 & Galb1-4GIcb-Sp8 & -19 & 11 & -55 \\
\hline 377 & Neu5Aca2-6Galb1-4GIcNAcb1-3GalNAc-Sp14 & -33 & 26 & -77 \\
\hline
\end{tabular}

Supporting data 5. rAAL-H59Q_200ug_16388_v5.1_DATA

\begin{tabular}{|c|c|c|c|c|}
\hline $\begin{array}{l}\text { Chart } \\
\text { Number }\end{array}$ & rAAL-H59Q 200ug/mI Slide\#16388 CFG\#2804 v5.1 Alexa488 5/27/13 HJ & Average RFU & StDev & $\% \mathrm{CV}$ \\
\hline 570 & (3S)GlcAb1-3Galb1-4GIcNAcb1-2Mana-Sp0 & 2139 & 62 & 3 \\
\hline 569 & (3S)GlcAb1-3Galb1-4GIcNAcb1-3Galb1-4Glc-Sp0 & 1330 & 53 & 4 \\
\hline 29 & (3S)Galb1-3GalNAca-Sp8 & 858 & 93 & 11 \\
\hline 35 & (3S)Galb1-4(6S)GIcNAcb-Sp8 & 156 & 22 & 14 \\
\hline 36 & (3S)Galb1-4GlcNAcb-Sp0 & 145 & 54 & 37 \\
\hline 25 & (3S)Galb1-4Glcb-Sp8 & 91 & 7 & 8 \\
\hline 26 & (3S)Galb1-4(6S)GIcb-Sp0 & 78 & 21 & 27 \\
\hline 30 & (3S)Galb1-3GIcNAcb-Sp0 & 58 & 13 & 22 \\
\hline 27 & (3S)Galb1-4(6S)Glcb-Sp8 & 53 & 27 & 50 \\
\hline 378 & Neu5Aca2-3Galb1-4(Fuca1-3)GlcNAcb1-3GalNAca-Sp14 & 42 & 9 & 23 \\
\hline 472 & $\begin{array}{l}\text { Fuca1-2Galb1-4(Fuca1-3)GlcNAcb1-2Mana1-6(Fuca1-2Galb1-4(Fuca1-3)GlcNAcb1-2Mana1-3)Manb1- } \\
\text { 4GIcNAcb1-4(Fuca1-6)GlcNAcb-Sp24 }\end{array}$ & 33 & 34 & 101 \\
\hline 572 & $\begin{array}{l}\text { Galb1-3GlcNAcb1-3Galb1-4GlcNAcb1-6(Galb1-3GlcNAcb1-3Galb1-4GlcNAb1-2)Mana1-6(Galb1-3GlcNAcb1- } \\
\text { 3Galb1-4GlcNAcb1-2Mana1-3)Manb1-4GlcNAcb1-4(Fuca1-6)GlcNAcb-Sp24 }\end{array}$ & 27 & 23 & 85 \\
\hline 325 & $\begin{array}{l}\text { Neu5Aca2-3Galb1-4GIcNAcb1-2Mana1-6(Neu5Aca2-3Galb1-4GIcNAcb1-2Mana1-3)Manb1-4GIcNAcb1- } \\
\text { 4GIcNAcb-Sp12 }\end{array}$ & 27 & 9 & 35 \\
\hline 583 & $\begin{array}{l}\text { GlcNAcb1-3Galb1-4GlcNAcb1-3Galb1-4GlcNAcb1-6(GlcNAcb1-3Galb1-4GlcNAcb1-3Galb1-4GlcNAb1-2)Mana1- } \\
\text { 6(GlcNAcb1-3Galb1-4GlcNAcb1-3Galb1-4GlcNAcb1-2Mana1-3)Manb1-4GlcNAcb1-4(Fuca1-6)GlcNAcb-Sp24 }\end{array}$ & 25 & 11 & 45 \\
\hline 34 & (3S)Galb1-4(6S)GIcNAcb-Sp0 & 24 & 15 & 63 \\
\hline 155 & Galb1-4(6S)Glcb-Sp0 & 24 & 9 & 39 \\
\hline 500 & Fuca1-2Galb1-3(6S)GIcNAcb-Sp0 & 23 & 9 & 38 \\
\hline 22 & 6S(3S)Galb1-4(6S)GIcNAcb-Sp0 & 23 & 15 & 67 \\
\hline 560 & Gala1-3Galb1-4GIcNAcb1-2Mana1-6(Gala1-3Galb1-4GlcNAcb1-2Mana1-3)Manb1-4GIcNAcb1-4GlcNAc-Sp24 & 22 & 17 & 75 \\
\hline
\end{tabular}




\begin{tabular}{|c|c|c|c|c|}
\hline 585 & $\begin{array}{l}\text { GlcNAcb1-3Galb1-4GlcNAcb1-3Galb1-4GIcNAcb1-3Galb1-4GlcNAcb1-6(GlcNAcb1-3Galb1-4GlcNAcb1-3Galb1- } \\
\text { 4GIcNAcb1-3Galb1-4GIcNAb1-2)Mana1-6(GIcNAcb1-3Galb1-4GlcNAcb1-3Galb1-4GIcNAcb1-3Galb1- } \\
\text { 4GIcNAcb1-2Mana1-3)Manb1-4GIcNAcb1-4(Fuca1-6)GIcNAcb-Sp24 }\end{array}$ & 22 & 6 & 28 \\
\hline 579 & $\begin{array}{l}\text { Galb1-4GIcNAcb1-3Galb1-4GlcNAcb1-3Galb1-4GlcNAcb1-3Galb1-4GIcNAcb1-2Mana1-6(Galb1-4GlcNAcb1- } \\
\text { 3Galb1-4GlcNAcb1-3Galb1-4GlcNAcb1-3Galb1-4GIcNAcb1-2Mana1-3)Manb1-4GlcNAcb1-4(Fuca1-6)GlcNAcb- } \\
\text { Sp24 }\end{array}$ & 21 & 7 & 35 \\
\hline 580 & $\begin{array}{l}\text { GlcNAcb1-3Galb1-4GlcNAcb1-3Galb1-4GlcNAcb1-3Galb1-4GlcNAcb1-3Galb1-4GlcNAcb1-2Mana1-6(GlcNAcb1- } \\
\text { 3Galb1-4GlcNAcb1-3Galb1-4GIcNAcb1-3Galb1-4GIcNAcb1-3Galb1-4GIcNAcb1-2Mana1-3)Manb1-4GlcNAcb1- } \\
\text { 4(Fuca1-6)GlcNAcb-Sp19 }\end{array}$ & 21 & 12 & 57 \\
\hline 372 & $\begin{array}{l}\text { Gala1-3Galb1-4(Fuca1-3)GlcNAcb1-2Mana1-6(Gala1-3Galb1-4(Fuca1-3)GlcNAcb1-2Mana1-3)Manb1- } \\
\text { 4GlcNAcb1-4GlcNAcb-Sp20 }\end{array}$ & 21 & 17 & 84 \\
\hline 192 & GlcNAcb1-6GalNAca-Sp8 & 20 & 10 & 53 \\
\hline 591 & Galb1-4GlcNAcb1-3Galb1-4GIcNAcb1-6(Galb1-4GIcNAcb1-3Galb1-4GIcNAcb1-3)GalNAca-Sp14 & 20 & 3 & 15 \\
\hline 586 & $\begin{array}{l}\text { Galb1-4GIcNAcb1-3Galb1-4GIcNAcb1-3Galb1-4GIcNAcb1-3Galb1-4GlcNAcb1-6(Galb1-4GlcNAcb1-3Galb1- } \\
\text { 4GIcNAcb1-3Galb1-4GIcNAcb1-3Galb1-4GlcNAb1-2)Mana1-6(Galb1-4GlcNAcb1-3Galb1-4GlcNAcb1-3Galb1- } \\
\text { 4GIcNAcb1-3Galb1-4GIcNAcb1-2Mana1-3)Manb1-4GIcNAcb1-4(Fuca1-6)GlcNAcb-Sp24 }\end{array}$ & 19 & 4 & 19 \\
\hline 543 & $\begin{array}{l}\text { Neu5Gca2-3Galb1-4GIcNAcb1-3Galb1-4GIcNAcb1-2Mana1-6(Neu5Gca2-3Galb1-4GIcNAcb1-3Galb1- } \\
\text { 4GIcNAcb1-2Mana1-3)Manb1-4GIcNAcb1-4GIcNAcb-Sp24 }\end{array}$ & 19 & 11 & 55 \\
\hline 440 & Galb1-6Galb-Sp10 & 19 & 29 & 149 \\
\hline 120 & Gala1-4Galb1-4GIcNAcb-Sp0 & 19 & 7 & 39 \\
\hline 11 & Neu5Acb-Sp8 & 19 & 11 & 57 \\
\hline 37 & (3S)Galb1-4GlcNAcb-Sp8 & 19 & 15 & 82 \\
\hline 121 & Gala1-4Galb1-4GlcNAcb-Sp8 & 18 & 9 & 51 \\
\hline 448 & Galb1-4GlcNAcb1-2Mana-Sp0 & 18 & 6 & 33 \\
\hline 420 & $\begin{array}{l}\text { Fuca1-2Galb1-4GIcNAcb1-2Mana1-6(Fuca1-2Galb1-4GIcNAcb1-2Mana1-3)Manb1-4GIcNAcb1-4(Fuca1- } \\
\text { 6)GIcNAcb-Sp22 }\end{array}$ & 18 & 16 & 89 \\
\hline 272 & Neu5Aca2-6Galb1-4GIcb-Sp0 & 18 & 4 & 20 \\
\hline 290 & Galb1-4(Fuca1-3)(6S)GIcNAcb-Sp0 & 18 & 11 & 60 \\
\hline 337 & Neu5Aca2-3Galb1-4(Fuca1-3)GlcNAcb1-6(Neu5Aca2-3Galb1-3)GalNAc-Sp14 & 18 & 8 & 47 \\
\hline 41 & (6P)Mana-Sp8 & 17 & 6 & 33 \\
\hline 565 & $\begin{array}{l}\text { Galb1-4GIcNAcb1-3Galb1-4GIcNAcb1-3Galb1-4GlcNAcb1-3Galb1-4GlcNAcb1-3Galb1-4GIcNAcb1-2Mana1- } \\
\text { 6(Galb1-4GlcNAcb1-3Galb1-4GIcNAcb1-3Galb1-4GIcNAcb1-3Galb1-4GIcNAcb1-3Galb1-4GIcNAcb1-2Mana1- } \\
\text { 3)Manb1-4GlcNAcb1-4GlcNAcb-Sp25 }\end{array}$ & 17 & 6 & 34 \\
\hline 195 & Glca1-4Glcb-Sp8 & 17 & 12 & 74 \\
\hline 588 & $\begin{array}{l}\text { Galb1-4GIcNAcb1-3Galb1-4GlcNAcb1-3Galb1-4GlcNAcb1-3Galb1-4GlcNAcb1-3Galb1-4GIcNAcb1-6(Galb1- } \\
\text { 4GlcNAcb1-3Galb1-4GlcNAcb1-3Galb1-4GlcNAcb1-3Galb1-4GIcNAcb1-3Galb1-4GlcNAb1-2)Mana1-6(Galb1- } \\
\text { 4GlcNAcb1-3Galb1-4GlcNAcb1-3Galb1-4GlcNAcb1-3Galb1-4GlcNAcb1-3Galb1-4GlcNAcb1-2Mana1-3)Manb1- } \\
\text { 4GlcNAcb1-4(Fuca1-6)GlcNAcb-Sp24 }\end{array}$ & 16 & 5 & 31 \\
\hline 380 & Galb1-3GalNAca1-3(Fuca1-2)Galb1-4Glc-Sp0 & 16 & 6 & 35 \\
\hline 346 & Mana1-6(Neu5Aca2-6Galb1-4GIcNAcb1-2Mana1-3)Manb1-4GIcNAcb1-4GIcNAc-Sp12 & 16 & 13 & 81 \\
\hline 545 & $\begin{array}{l}\text { GlcNAcb1-3Galb1-4GlcNAcb1-3Galb1-4GIcNAcb1-2Mana1-6(GlcNAcb1-3Galb1-4GIcNAcb1-3Galb1-4GlcNAcb1- } \\
\text { 2Mana1-3)Manb1-4GIcNAcb1-4GIcNAcb-Sp12 }\end{array}$ & 16 & 7 & 42 \\
\hline 407 & Galb1-3GIcNAca1-6Galb1-4GIcNAcb-Sp0 & 15 & 7 & 45 \\
\hline
\end{tabular}




\begin{tabular}{|c|c|c|c|c|}
\hline 364 & Galb1-4GIcNAcb1-2Mana1-6(Mana1-3)Manb1-4GIcNAcb1-4GIcNAcb-Sp12 & 15 & 6 & 41 \\
\hline 132 & Galb1-4GIcNAcb1-6GalNAc-Sp14 & 15 & 6 & 41 \\
\hline 334 & GalNAcb1-3Gala1-4Galb1-4GlcNAcb1-3Galb1-4Glcb-Sp0 & 15 & 14 & 91 \\
\hline 419 & $\begin{array}{l}\text { Galb1-4(Fuca1-3)GIcNAcb1-2Mana1-6(Galb1-4(Fuca1-3)GIcNAcb1-2Mana1-3)Manb1-4GIcNAcb1-4(Fuca1- } \\
\text { 6)GIcNAcb-Sp22 }\end{array}$ & 15 & 6 & 41 \\
\hline 53 & GlcNAcb1-2Mana1-6(GlcNAcb1-2Mana1-3)Manb1-4GlcNAcb1-4GlcNAcb-Sp13 & 15 & 10 & 64 \\
\hline 582 & $\begin{array}{l}\text { Galb1-4GIcNAcb1-3Galb1-4GlcNAcb1-6(Galb1-4GlcNAcb1-3Galb1-4GIcNAb1-2)Mana1-6(Galb1-4GlcNAcb1- } \\
\text { 3Galb1-4GIcNAcb1-2Mana1-3)Manb1-4GlcNAcb1-4(Fuca1-6)GlcNAcb-Sp24 }\end{array}$ & 15 & 7 & 47 \\
\hline 564 & $\begin{array}{l}\text { GlcNAcb1-3Galb1-4GIcNAcb1-3Galb1-4GIcNAcb1-3Galb1-4GIcNAcb1-3Galb1-4GIcNAcb1-2Mana1-6(GlcNAcb1- } \\
\text { 3Galb1-4GIcNAcb1-3Galb1-4GIcNAcb1-3Galb1-4GIcNAcb1-3Galb1-4GIcNAcb1-2Mana1-3)Manb1-4GIcNAcb1- } \\
\text { 4GIcNAcb-Sp25 }\end{array}$ & 15 & 13 & 87 \\
\hline 484 & Mana1-6(Mana1-3)Manb1-4GIcNAcb1-4(Fuca1-6)GlcNAcb-Sp19 & 15 & 10 & 71 \\
\hline 511 & (6S)(4S)GalNAcb1-4GIcNAc-Sp8 & 14 & 4 & 28 \\
\hline 228 & GalNAcb1-4(Neu5Aca2-8Neu5Aca2-3)Galb1-4Glcb-Sp0 & 14 & 6 & 43 \\
\hline 512 & (6S)GalNAcb1-4GlcNAc-Sp8 & 14 & 13 & 95 \\
\hline 208 & Mana1-2Mana1-6(Mana1-2Mana1-3)Mana-Sp9 & 14 & 8 & 54 \\
\hline 600 & Neu5Aca2-3Galb1-4GIcNAcb1-3Galb1-4GIcNAcb1-6(Galb1-3)GalNAca-Sp14 & 14 & 5 & 33 \\
\hline 353 & Galb1-4GlcNAcb1-2Mana1-6(Galb1-4GlcNAcb1-2Mana1-3)Manb1-4GlcNAcb1-4(Fuca1-6)GlcNAcb-Sp22 & 14 & 5 & 34 \\
\hline 606 & $\begin{array}{l}\text { Neu5Aca2-6Galb1-4GIcNAcb1-3Galb1-4GIcNAcb1-3Galb1-4GIcNAcb1-2Mana1-6(Neu5Aca2-6Galb1- } \\
\text { 4GlcNAcb1-3Galb1-4GIcNAcb1-3Galb1-4GIcNAcb1-2Mana1-3)Manb1-4GIcNAcb1-4GlcNAcb-Sp12 }\end{array}$ & 14 & 7 & 52 \\
\hline 99 & GalNAcb1-4GIcNAcb-Sp8 & 14 & 12 & 89 \\
\hline 530 & GlcNAcb1-2 Mana1-6(GIcNAcb1-4)(GlcNAcb1-2Mana1-3)Manb1-4GIcNAcb1-4(Fuca1-6)GIcNAc-Sp21 & 14 & 12 & 83 \\
\hline 116 & Gala1-3Galb1-4Glcb-Sp0 & 14 & 3 & 18 \\
\hline 482 & $\begin{array}{l}\text { Neu5Aca2-6Galb1-4GIcNAcb1-2Mana1-6(Neu5Aca2-6Galb1-4GIcNAcb1-2Mana1-3)Manb1-4GlcNAcb1-4(Fuca1- } \\
\text { 6)GIcNAcb-Sp24 }\end{array}$ & 14 & 5 & 34 \\
\hline 64 & Fuca1-2Galb1-3GalNAcb1-4(Neu5Aca2-3)Galb1-4Glcb-Sp9 & 14 & 8 & 58 \\
\hline 96 & GalNAcb1-3Gala1-4Galb1-4GIcNAcb-Sp0 & 14 & 6 & 47 \\
\hline 387 & $\begin{array}{l}\text { Galb1-4GIcNAcb1-6(Galb1-4GIcNAcb1-2)Mana1-6(Galb1-4GlcNAcb1-4(Galb1-4GIcNAcb1-2)Mana1-3)Manb1- } \\
\text { 4GIcNAcb1-4GIcNAcb-Sp21 }\end{array}$ & 14 & 3 & 19 \\
\hline 31 & (3S)Galb1-3GlcNAcb-Sp8 & 14 & 7 & 49 \\
\hline 261 & Neu5Aca2-3Galb1-4GIcNAcb1-3Galb1-4GIcNAcb-Sp0 & 14 & 4 & 30 \\
\hline 501 & Fuca1-2(6S)Galb1-3(6S)GIcNAcb-Sp0 & 14 & 4 & 32 \\
\hline 375 & $\begin{array}{l}\text { Fuca1-4(Fuca1-2Galb1-3)GIcNAcb1-2Mana1-3(Fuca1-4(Fuca1-2Galb1-3)GlcNAcb1-2Mana1-3)Manb1- } \\
\text { 4GIcNAcb1-4GlcNAcb-Sp19 }\end{array}$ & 13 & 7 & 54 \\
\hline 395 & $\begin{array}{l}\text { Gala1-3Galb1-3(Fuca1-4)GlcNAcb1-2Mana1-6(Gala1-3Galb1-3(Fuca1-4)GlcNAcb1-2Mana1-3)Manb1- } \\
\text { 4GIcNAcb1-4GlcNAc-Sp19 }\end{array}$ & 13 & 6 & 45 \\
\hline 562 & GalNAcb1-3GlcNAcb-Sp0 & 13 & 7 & 51 \\
\hline 575 & $\begin{array}{l}\text { Galb1-4GIcNAcb1-3Galb1-4GIcNAcb1-2Mana1-6(Galb1-4GlcNAcb1-3Galb1-4GlcNAcb1-2Mana1-3)Manb1- } \\
\text { 4GlcNAcb1-4(Fuca1-6)GIcNAcb-Sp24 }\end{array}$ & 13 & 6 & 46 \\
\hline 4 & GalNAca-Sp8 & 13 & 12 & 89 \\
\hline 393 & GalNAca1-3(Fuca1-2)Galb1-3GalNAca1-3(Fuca1-2)Galb1-4GIcNAcb-Sp0 & 13 & 11 & 85 \\
\hline 91 & GalNAca1-3GalNAcb-Sp8 & 13 & 3 & 26 \\
\hline
\end{tabular}




\begin{tabular}{|c|c|c|c|c|}
\hline 473 & $\begin{array}{l}\text { Fuca1-2Galb1-3(Fuca1-4)GIcNAcb1-2Mana1-6(Fuca1-2Galb1-3(Fuca1-4)GIcNAcb1-2Mana1-3)Manb1- } \\
\text { 4GIcNAcb1-4(Fuca1-6)GIcNAcb1-4(Fuca1-6)GlcNAcb-Sp19 }\end{array}$ & 13 & 3 & 24 \\
\hline 460 & $\begin{array}{l}\text { Neu5Aca2-3Galb1-4GIcNAcb1-4Mana1-6(GlcNAcb1-4)(Neu5Aca2-3Galb1-4GlcNAcb1-4(Neu5Aca2-3Galb1- } \\
\text { 4GIcNAcb1-2)Mana1-3)Manb1-4GIcNAcb1-4GIcNAcb-Sp21 }\end{array}$ & 13 & 10 & 75 \\
\hline 485 & $\begin{array}{l}\text { Galb1-4GIcNAcb1-6(Galb1-4GIcNAcb1-2)Mana1-6(Galb1-4GIcNAcb1-2Mana1-3)Manb1-4GIcNAcb1-4(Fuca1- } \\
\text { 6)GIcNAcb-Sp24 }\end{array}$ & 13 & 8 & 61 \\
\hline 257 & Neu5Aca2-3Galb1-4(Fuca1-3)GlcNAcb1-3Galb1-4GlcNAcb-Sp8 & 13 & 12 & 88 \\
\hline 82 & GalNAca1-3(Fuca1-2)Galb1-3GIcNAcb-Sp0 & 13 & 6 & 47 \\
\hline 431 & GlcNAcb1-2Mana1-6(GlcNAcb1-4)(GlcNAcb1-2Mana1-3)Manb1-4GIcNAcb1-4GIcNAc-Sp21 & 13 & 7 & 53 \\
\hline 576 & $\begin{array}{l}\text { GlcNAcb1-3Galb1-4GIcNAcb1-3Galb1-4GIcNAcb1-2Mana1-6(GIcNAcb1-3Galb1-4GIcNAcb1-3Galb1-4GIcNAcb1- } \\
\text { 2Mana1-3)Manb1-4GlcNAcb1-4(Fuca1-6)GIcNAcb-Sp24 }\end{array}$ & 13 & 18 & 143 \\
\hline 143 & Galb1-3GalNAcb1-3Gala1-4Galb1-4Glcb-Sp0 & 13 & 4 & 31 \\
\hline 399 & Neu5Aca2-3Galb1-3GIcNAcb1-3GalNAca-Sp14 & 13 & 5 & 43 \\
\hline 66 & Fuca1-2Galb1-3GlcNAcb1-3Galb1-4Glcb-Sp10 & 13 & 4 & 30 \\
\hline 595 & GlcNAcb1-3Galb1-4GlcNAcb1-6(GlcNAcb1-3Galb1-4GlcNAcb1-3)GalNAca-Sp14 & 13 & 5 & 37 \\
\hline 142 & Galb1-3GalNAcb-Sp8 & 13 & 10 & 80 \\
\hline 357 & KDNa2-6Galb1-4GIcNAc-Sp0 & 12 & 5 & 43 \\
\hline 514 & GalNAcb1-4(6S)GlcNAc-Sp8 & 12 & 9 & 75 \\
\hline 488 & Galb1-3GIcNAcb1-6GalNAca-Sp14 & 12 & 4 & 36 \\
\hline 68 & Fuca1-2Galb1-3GIcNAcb-Sp8 & 12 & 3 & 28 \\
\hline 186 & GlcNAcb1-4-MDPLys & 12 & 11 & 89 \\
\hline 104 & Gala1-3(Fuca1-2)Galb1-4(Fuca1-3)GlcNAcb-Sp8 & 12 & 10 & 81 \\
\hline 226 & GalNAcb1-4(Neu5Aca2-8Neu5Aca2-8Neu5Aca2-3)Galb1-4Glcb-Sp0 & 12 & 12 & 94 \\
\hline 315 & Mana1-2Mana1-6(Mana1-3)Mana1-6(Mana1-2Mana1-2Mana1-3)Mana-Sp9 & 12 & 6 & 53 \\
\hline 382 & Galb1-3GIcNAcb1-3Galb1-4GIcNAcb1-6(Galb1-3GlcNAcb1-3)Galb1-4Glcb-Sp0 & 12 & 8 & 64 \\
\hline 105 & Gala1-3(Fuca1-2)Galb1-4GlcNAc-Sp0 & 12 & 8 & 64 \\
\hline 21 & GlcNAcb1-6(GlcNAcb1-4)(GlcNAcb1-3)GlcNAc-Sp8 & 12 & 10 & 82 \\
\hline 203 & GlcAb1-3Galb-Sp8 & 12 & 14 & 114 \\
\hline 418 & GalNAca1-3(Fuca1-2)Galb1-4(Fuca1-3)GlcNAcb1-3GalNAc-Sp14 & 12 & 5 & 45 \\
\hline 323 & Neu5Gcb2-6Galb1-4GlcNAc-Sp8 & 12 & 12 & 99 \\
\hline 216 & Mana1-6(Mana1-3)Mana1-6(Mana1-3)Manb1-4GIcNAcb1-4GIcNAcb-Sp12 & 12 & 15 & 130 \\
\hline 607 & $\begin{array}{l}\text { Neu5Aca2-3Galb1-4GIcNAcb1-3Galb1-4GIcNAcb1-3Galb1-4GlcNAcb1-2Mana1-6(Neu5Aca2-3Galb1- } \\
\text { 4GIcNAcb1-3Galb1-4GlcNAcb1-3Galb1-4GlcNAcb1-2Mana1-3)Manb1-4GIcNAcb1-4GlcNAcb-Sp12 }\end{array}$ & 12 & 13 & 109 \\
\hline 88 & GlcNAcb1-3Galb1-3GalNAca-Sp8 & 12 & 8 & 66 \\
\hline 478 & Neu5Aca2-3Galb1-4GlcNAcb1-2Mana-Sp0 & 12 & 7 & 60 \\
\hline 108 & Gala1-3(Fuca1-2)Galb-Sp18 & 12 & 6 & 50 \\
\hline 593 & GlcNAcb1-3Galb1-4GIcNAcb1-3GalNAca-Sp14 & 12 & 6 & 51 \\
\hline 594 & GlcNAcb1-3Galb1-4GIcNAcb1-6(Galb1-3)GalNAca-Sp14 & 12 & 6 & 52 \\
\hline 344 & GIcNAca1-4Galb1-3GalNAc-Sp14 & 12 & 7 & 60 \\
\hline 110 & Gala1-3GalNAca-Sp8 & 12 & 4 & 32 \\
\hline 458 & $\begin{array}{l}\text { Galb1-4GIcNAcb1-6(Galb1-4GIcNAcb1-2)Mana1-6(Galb1-4GIcNAcb1-2Mana1-3)Manb1-4GIcNAcb1-4GIcNAcb- } \\
\text { Sp19 }\end{array}$ & 11 & 7 & 65 \\
\hline 481 & Neu5Aca2-6Galb1-4 GlcNAcb1-6(Neu5Aca2-6Galb1-4GIcNAcb1-3)GalNAca-Sp14 & 11 & 10 & 85 \\
\hline
\end{tabular}




\begin{tabular}{|c|c|c|c|c|}
\hline 350 & Galb1-4GIcNAcb1-2Mana1-6Manb1-4GIcNAcb1-4GIcNAc-Sp12 & 11 & 4 & 36 \\
\hline 65 & Fuca1-2Galb1-3GlcNAcb1-3Galb1-4Glcb-Sp8 & 11 & 6 & 56 \\
\hline 131 & Galb1-4GIcNAcb1-6GalNAca-Sp8 & 11 & 10 & 92 \\
\hline 362 & $\begin{array}{l}\text { Fuca1-2Galb1-4(Fuca1-3)GlcNAcb1-2Mana1-6(Fuca1-2Galb1-4(Fuca1-3)GlcNAcb1-2Mana1-3)Manb1- } \\
\text { 4GlcNAcb1-4GlcNAb-Sp20 }\end{array}$ & 11 & 1 & 13 \\
\hline 288 & Neu5Aca2-3Galb1-4GIcNAcb1-6(Galb1-3)GalNAca-Sp14 & 11 & 6 & 51 \\
\hline 424 & GalNAca1-3(Fuca1-2)Galb1-3GlcNAcb1-3GalNAc-Sp14 & 11 & 3 & 23 \\
\hline 55 & $\begin{array}{l}\text { Neu5Aca2-6Galb1-4GIcNAcb1-2Mana1-6(Neu5Aca2-6Galb1-4GIcNAcb1-2Mana1-3)Manb1-4GIcNAcb1- } \\
\text { 4GIcNAcb-Sp12 }\end{array}$ & 11 & 10 & 94 \\
\hline 605 & $\begin{array}{l}\text { Neu5Aca2-6Galb1-4GIcNAcb1-3Galb1-4GIcNAcb1-6(Neu5Aca2-6Galb1-4GIcNAcb1-3Galb1-4GIcNAcb1- } \\
\text { 3)GalNAca-Sp14 }\end{array}$ & 11 & 4 & 38 \\
\hline 520 & Neu5Aca2-6Galb1-4GIcNAcb1-2Man-Sp0 & 11 & 4 & 40 \\
\hline 200 & G-ol-Sp8 & 11 & 7 & 60 \\
\hline 587 & $\begin{array}{l}\text { GlcNAcb1-3Galb1-4GlcNAcb1-3Galb1-4GIcNAcb1-3Galb1-4GlcNAcb1-3Galb1-4GlcNAcb1-6(GlcNAcb1-3Galb1- } \\
\text { 4GlcNAcb1-3Galb1-4GlcNAcb1-3Galb1-4GIcNAcb1-3Galb1-4GIcNAb1-2)Mana1-6(GlcNAcb1-3Galb1- } \\
\text { 4GlcNAcb1-3Galb1-4GIcNAcb1-3Galb1-4GlcNAcb1-3Galb1-4GlcNAcb1-2Mana1-3)Manb1-4GIcNAcb1-4(Fuca1- } \\
\text { 6)GlcNAcb-Sp24 }\end{array}$ & 11 & 10 & 88 \\
\hline 355 & (6S)GlcNAcb1-3Galb1-4GlcNAcb-Sp0 & 11 & 6 & 57 \\
\hline 196 & Glca1-4Glca-Sp8 & 11 & 3 & 27 \\
\hline 578 & $\begin{array}{l}\text { GlcNAcb1-3Galb1-4GlcNAcb1-3Galb1-4GIcNAcb1-3Galb1-4GIcNAcb1-2Mana1-6(GlcNAcb1-3Galb1-4GIcNAcb1- } \\
\text { 3Galb1-4GIcNAcb1-3Galb1-4GlcNAcb1-2Mana1-3)Manb1-4GIcNAcb1-4(Fuca1-6)GlcNAcb-Sp24 }\end{array}$ & 11 & 9 & 81 \\
\hline 425 & Gala1-3Galb1-3GIcNAcb1-3GalNAc-Sp14 & 11 & 4 & 37 \\
\hline 360 & Fuca1-2Galb1-3GIcNAcb1-2Mana1-6(Fuca1-2Galb1-3GlcNAcb1-2Mana1-3)Manb1-4GIcNAcb1-4GlcNAcb-Sp20 & 11 & 2 & 18 \\
\hline 552 & Neu5Gca2-8Neu5Gca2-3Galb1-4GIcNAc-Sp0 & 11 & 10 & 93 \\
\hline 469 & Glca1-6Glca1-6Glca1-6Glcb-Sp10 & 11 & 5 & 51 \\
\hline 489 & Gala1-3Galb1-3GlcNAcb1-6GalNAca-Sp14 & 11 & 7 & 63 \\
\hline 165 & Galb1-4GIcNAcb1-3Galb1-4Glcb-Sp8 & 11 & 9 & 83 \\
\hline 502 & Neu5Aca2-6GalNAcb1-4(6S)GIcNAcb-Sp8 & 11 & 6 & 54 \\
\hline 450 & Gala1-3(Fuca1-2)Galb1-4GIcNAcb1-6(Gala1-3(Fuca1-2)Galb1-4GIcNAcb1-3)GalNAc-Sp14 & 11 & 4 & 42 \\
\hline 442 & GalNAcb1-6GalNAcb-Sp8 & 11 & 8 & 79 \\
\hline 61 & Fuca1-2Galb1-3GalNAca-Sp8 & 11 & 8 & 76 \\
\hline 574 & $\begin{array}{l}\text { GlcNAcb1-3Galb1-4GlcNAcb1-2Mana1-6(GlcNAcb1-3Galb1-4GIcNAcb1-2Mana1-3)Manb1-4GIcNAcb1-4(Fuca1- } \\
\text { 6)GlcNAcb-Sp24 }\end{array}$ & 10 & 9 & 82 \\
\hline 513 & (3S)GalNAcb1-4(3S)GlcNAc-Sp8 & 10 & 7 & 63 \\
\hline 169 & Galb1-4GlcNAcb-Sp8 & 10 & 10 & 92 \\
\hline 475 & GlcNAcb1-6(GlcNAcb1-2)Mana1-6(GlcNAcb1-2Mana1-3)Manb1-4GIcNAcb1-4(Fuca1-6)GlcNAcb-Sp24 & 10 & 11 & 102 \\
\hline 558 & $\begin{array}{l}\text { GlcNAcb1-3Galb1-4GIcNAcb1-6(GIcNAcb1-3Galb1-4GlcNAcb1-2)Mana1-6(GlcNAcb1-3Galb1-4GIcNAcb1- } \\
\text { 2Man a1-3)Manb1-4GlcNAcb1-4GIcNAc-Sp24 }\end{array}$ & 10 & 6 & 61 \\
\hline 547 & $\begin{array}{l}\text { Galb1-4GIcNAcb1-3Galb1-4GIcNAcb1-3Galb1-4GIcNAcb1-2Mana1-6(Galb1-4GlcNAcb1-3Galb1-4GlcNAcb1- } \\
\text { 3Galb1-4GIcNAcb1-2Mana1-3)Manb1-4GIcNAcb1-4GIcNAcb-Sp12 }\end{array}$ & 10 & 5 & 47 \\
\hline 8 & Rhaa-Sp8 & 10 & 3 & 28 \\
\hline 433 & GlcNAcb1-6(GlcNAcb1-2)Mana1-6(GlcNAcb1-4)(GlcNAcb1-2Mana1-3)Manb1-4GlcNAcb1-4GlcNAc-Sp21 & 10 & 7 & 70 \\
\hline 97 & GalNAcb1-4(Fuca1-3)GIcNAcb-Sp0 & 10 & 6 & 57 \\
\hline
\end{tabular}




\begin{tabular}{|c|c|c|c|c|}
\hline 84 & (3S)Galb1-4(Fuca1-3)Glcb-Sp0 & 10 & 5 & 50 \\
\hline 492 & (3S)Galb1-3(Fuca1-4)GIcNAcb-Sp0 & 10 & 6 & 64 \\
\hline 119 & Gala1-4(Fuca1-2)Galb1-4GlcNAcb-Sp8 & 10 & 5 & 47 \\
\hline 491 & Neu5Aca2-3Galb1-3GIcNAcb1-6GalNAca-Sp14 & 10 & 4 & 38 \\
\hline 383 & Galb1-4(Fuca1-3)GIcNAcb1-6(Galb1-3GIcNAcb1-3)Galb1-4GIc-Sp21 & 10 & 6 & 58 \\
\hline 156 & Galb1-4(6S)Glcb-Sp8 & 10 & 10 & 95 \\
\hline 584 & $\begin{array}{l}\text { Galb1-4GIcNAcb1-3Galb1-4GIcNAcb1-3Galb1-4GIcNAcb1-6(Galb1-4GIcNAcb1-3Galb1-4GlcNAcb1-3Galb1- } \\
\text { 4GIcNAb1-2)Mana1-6(Galb1-4GIcNAcb1-3Galb1-4GIcNAcb1-3Galb1-4GIcNAcb1-2Mana1-3)Manb1-4GIcNAcb1- } \\
\text { 4(Fuca1-6)GlcNAcb-Sp24 }\end{array}$ & 10 & 9 & 85 \\
\hline 304 & Galb1-4GlcNAca1-6Galb1-4GIcNAcb-Sp0 & 10 & 12 & 121 \\
\hline 446 & $\begin{array}{l}\text { Fuca1-2Galb1-4(Fuca1-3)GlcNAcb1-2Mana1-6(Fuca1-2Galb1-4(Fuca1-3)GlcNAcb1-4(Fuca1-2Galb1-4(Fuca1- } \\
\text { 3)GlcNAcb1-2)Mana1-3)Manb1-4GlcNAcb1-4GlcNAcb-Sp12 }\end{array}$ & 10 & 8 & 86 \\
\hline 416 & Fuca1-2Galb1-4(Fuca1-3)GIcNAcb1-3GalNAca-Sp14 & 10 & 11 & 108 \\
\hline 342 & GlcNAca1-4Galb1-4GIcNAcb1-3Galb1-4(Fuca1-3)GlcNAcb1-3Galb1-4(Fuca1-3)GlcNAcb-Sp0 & 10 & 2 & 23 \\
\hline 377 & Neu5Aca2-6Galb1-4GIcNAcb1-3GalNAc-Sp14 & 10 & 4 & 37 \\
\hline 51 & Mana1-6(Mana1-3)Manb1-4GlcNAcb1-4GlcNAcb-Sp13 & 10 & 8 & 88 \\
\hline 202 & GlcAb-Sp8 & 10 & 7 & 72 \\
\hline 503 & GalNAcb1-4(Fuca1-3)(6S)GlcNAcb-Sp8 & 10 & 9 & 96 \\
\hline 396 & $\begin{array}{l}\text { Neu5Aca2-3Galb1-3GIcNAcb1-2Mana1-6(Neu5Aca2-3Galb1-3GIcNAcb1-2Mana1-3)Manb1-4GIcNAcb1- } \\
\text { 4GIcNAc-Sp19 }\end{array}$ & 10 & 6 & 66 \\
\hline 146 & Galb1-3Galb-Sp8 & 10 & 4 & 38 \\
\hline 590 & Galb1-4GIcNAcb1-3Galb1-4GIcNAcb1-6(Galb1-3)GalNAca-Sp14 & 9 & 16 & 172 \\
\hline 256 & Neu5Aca2-3Galb1-4(Fuca1-3)GlcNAcb1-3Galb-Sp8 & 9 & 1 & 12 \\
\hline 356 & KDNa2-3Galb1-4(Fuca1-3)GıNAc-Sp0 & 9 & 9 & 98 \\
\hline 366 & Neu5Aca2-6GIcNAcb1-4GlcNAc-Sp21 & 9 & 8 & 86 \\
\hline 311 & GlcNAcb1-4GIcNAcb-Sp12 & 9 & 9 & 94 \\
\hline 2 & Glca-Sp8 & 9 & 7 & 77 \\
\hline 158 & Galb1-4GalNAcb1-3(Fuca1-2)Galb1-4GIcNAcb-Sp8 & 9 & 3 & 28 \\
\hline 38 & (3S)Galb-Sp8 & 9 & 5 & 54 \\
\hline 537 & Gala1-3(Fuca1-2)Galb1-3GalNAcb1-3Gala1-4Galb1-4GIc-Sp21 & 9 & 7 & 80 \\
\hline 81 & Fucb1-3GIcNAcb-Sp8 & 9 & 8 & 89 \\
\hline 336 & GalNAca1-3(Fuca1-2)Galb1-4GIcNAcb1-3Galb1-4GIcNAcb1-3Galb1-4GIcNAcb-Sp0 & 9 & 14 & 150 \\
\hline 610 & Galb1-3GalNAcb1-4(Neu5Aca2-8Neu5Aca2-8Neu5Aca2-3)Galb1-4GIcb-Sp21 & 9 & 6 & 63 \\
\hline 554 & Neu5Gca2-8Neu5Aca2-3Galb1-4GIcNAc-Sp0 & 9 & 3 & 30 \\
\hline 225 & GalNAcb1-4(Neu5Aca2-8Neu5Aca2-8Neu5Aca2-8Neu5Aca2-3)Galb1-4Glcb-Sp0 & 9 & 7 & 78 \\
\hline 175 & GlcNAcb1-2Galb1-3GalNAca-Sp8 & 9 & 3 & 38 \\
\hline 236 & Neu5Aca2-3GalNAca-Sp8 & 9 & 4 & 47 \\
\hline 339 & GlcNAca1-4Galb1-4GlcNAcb-Sp0 & 9 & 7 & 80 \\
\hline 329 & Neu5,9Ac2a2-3Galb1-3GlcNAcb-Sp0 & 9 & 2 & 27 \\
\hline 454 & $\begin{array}{l}\text { GalNAca1-3(Fuca1-2)Galb1-4GIcNAcb1-2Mana1-6(GalNAca1-3(Fuca1-2)Galb1-4GlcNAcb1-2Mana1-3)Manb1- } \\
\text { 4GIcNAcb1-4(Fuca1-6)GIcNAcb-Sp22 }\end{array}$ & 9 & 6 & 63 \\
\hline 427 & $\begin{array}{l}\text { Gala1-3(Fuca1-2)Galb1-4GlcNAcb1-2Mana1-6(Gala1-3(Fuca1-2)Galb1-4GlcNAcb1-2Mana1-3)Manb1- } \\
\text { 4GlcNAcb1-4(Fuca1-6)GlcNAcb-Sp22 }\end{array}$ & 9 & 4 & 44 \\
\hline
\end{tabular}




\begin{tabular}{|c|c|c|c|c|}
\hline 182 & GlcNAcb1-3Galb1-4GlcNAcb-Sp0 & 9 & 9 & 97 \\
\hline 432 & GlcNAcb1-2Mana1-6(GlcNAcb1-4)(GIcNAcb1-4(GlcNAcb1-2)Mana1-3)Manb1-4GlcNAcb1-4GlcNAc-Sp21 & 9 & 6 & 65 \\
\hline 215 & Mana1-6(Mana1-3)Mana1-6(Mana1-2Mana1-3)Manb1-4GlcNAcb1-4GlcNAcb-Sp12 & 9 & 9 & 105 \\
\hline 365 & $\begin{array}{l}\text { Fuca1-4(Galb1-3)GlcNAcb1-2Mana1-6(Fuca1-4(Galb1-3)GlcNAcb1-2Mana1-3)Manb1-4GlcNAcb1-4(Fuca1- } \\
\text { 6)GlcNAcb-Sp22 }\end{array}$ & 9 & 6 & 66 \\
\hline 80 & Fuca1-4GIcNAcb-Sp8 & 9 & 3 & 35 \\
\hline 333 & Gala1-4Galb1-4GIcNAcb1-3Galb1-4GIcb-Sp0 & 9 & 2 & 25 \\
\hline 83 & GalNAca1-3(Fuca1-2)Galb1-4(Fuca1-3)GIcNAcb-Sp0 & 9 & 4 & 41 \\
\hline 28 & (3S)Galb1-3(Fuca1-4)GIcNAcb-Sp8 & 9 & 1 & 13 \\
\hline 211 & Mana1-2Mana1-6(Mana1-3)Mana1-6(Mana1-2Mana1-2Mana1-3)Manb1-4GIcNAcb1-4GIcNAcb-Sp12 & 9 & 8 & 90 \\
\hline 70 & Fuca1-2Galb1-4(Fuca1-3)GlcNAcb1-3Galb1-4(Fuca1-3)GlcNAcb1-3Galb1-4(Fuca1-3)GlcNAcb-Sp0 & 9 & 4 & 43 \\
\hline 351 & Mana1-6(Galb1-4GIcNAcb1-2Mana1-3)Manb1-4GIcNAcb1-4GIcNAcb-Sp12 & 9 & 10 & 113 \\
\hline 347 & Neu5Aca2-6Galb1-4GIcNAcb1-2Mana1-6Manb1-4GIcNAcb1-4GIcNAc-Sp12 & 9 & 1 & 17 \\
\hline 341 & GIcNAca1-4Galb1-4GlcNAcb1-3Galb1-4Glcb-Sp0 & 9 & 6 & 76 \\
\hline 214 & Mana1-2Mana1-2Mana1-6(Mana1-3)Mana-Sp9 & 8 & 4 & 48 \\
\hline 435 & Galb1-4GIcNAcb1-2Mana1-6(GlcNAcb1-4)(Galb1-4GIcNAcb1-2Mana1-3)Manb1-4GIcNAcb1-4GIcNAc-Sp21 & 8 & 8 & 91 \\
\hline 394 & Gala1-3Galb1-3GIcNAcb1-2Mana1-6(Gala1-3Galb1-3GIcNAcb1-2Mana1-3)Manb1-4GIcNAcb1-4GlcNAc-Sp19 & 8 & 2 & 28 \\
\hline 338 & GlcNAca1-4Galb1-4GIcNAcb1-3Galb1-4GlcNAcb1-3Galb1-4GIcNAcb-Sp0 & 8 & 3 & 38 \\
\hline 322 & Neu5Aca2-8Neu5Aca2-8Neu5Acb-Sp8 & 8 & 8 & 93 \\
\hline 474 & $\begin{array}{l}\text { Neu5Aca2-3Galb1-3GIcNAcb1-6(Neu5Aca2-3Galb1-3GIcNAcb1-2)Mana1-6(Neu5Aca2-3Galb1-3GIcNAcb1- } \\
\text { 2Mana1-3)Manb1-4GIcNAcb1-4GIcNAcb-Sp19 }\end{array}$ & 8 & 9 & 108 \\
\hline 417 & Gala1-3(Fuca1-2)Galb1-4(Fuca1-3)GIcNAcb1-3GalNAc-Sp14 & 8 & 6 & 71 \\
\hline 518 & (6P)Galb1-4GlcNAcb-SP0 & 8 & 5 & 55 \\
\hline 86 & GalNAca1-3(Fuca1-2)Galb1-4GlcNAcb-Sp8 & 8 & 4 & 45 \\
\hline 95 & GalNAcb1-3(Fuca1-2)Galb-Sp8 & 8 & 6 & 74 \\
\hline 124 & Gala1-6Glcb-Sp8 & 8 & 10 & 118 \\
\hline 71 & Fuca1-2Galb1-4(Fuca1-3)GIcNAcb-Sp0 & 8 & 5 & 63 \\
\hline 239 & Neu5Aca2-3Galb1-3(Fuca1-4)GlcNAcb-Sp8 & 8 & 4 & 46 \\
\hline 1 & Gala-Sp8 & 8 & 4 & 53 \\
\hline 361 & Fuca1-2Galb1-4GlcNAcb1-2Mana1-6(Fuca1-2Galb1-4GlcNAcb1-2Mana1-3)Manb1-4GIcNAcb1-4GIcNAcb-Sp20 & 8 & 6 & 76 \\
\hline 373 & $\begin{array}{l}\text { GalNAca1-3(Fuca1-2)Galb1-3GlcNAcb1-2Mana1-6(GalNAca1-3(Fuca1-2)Galb1-3GlcNAcb1-2Mana1-3)Manb1- } \\
\text { 4GlcNAcb1-4GlcNAcb-Sp20 }\end{array}$ & 8 & 2 & 21 \\
\hline 94 & GalNAcb1-3GalNAca-Sp8 & 8 & 11 & 138 \\
\hline 224 & Neu5Aca2-3Galb1-3GalNAca-Sp14 & 8 & 7 & 92 \\
\hline 69 & Fuca1-2Galb1-4(Fuca1-3)GIcNAcb1-3Galb1-4(Fuca1-3)GIcNAcb-Sp0 & 8 & 10 & 119 \\
\hline 467 & Gala1-3(Fuca1-2)Galb1-3GalNAca-Sp8 & 8 & 8 & 93 \\
\hline 93 & GalNAca1-4(Fuca1-2)Galb1-4GlcNAcb-Sp8 & 8 & 6 & 71 \\
\hline 238 & Neu5Aca2-3Galb1-3(6S)GIcNAc-Sp8 & 8 & 4 & 44 \\
\hline 204 & GlcAb1-6Galb-Sp8 & 8 & 5 & 57 \\
\hline 76 & Fuca1-2Galb1-4GIcNAcb-Sp8 & 8 & 4 & 56 \\
\hline 403 & Gala1-4Galb1-3GIcNAcb1-2Mana1-6(Gala1-4Galb1-3GIcNAcb1-2Mana1-3)Manb1-4GIcNAcb1-4GlcNAcb-Sp19 & 8 & 11 & 136 \\
\hline 109 & Gala1-4(Gala1-3)Galb1-4GIcNAcb-Sp8 & 8 & 3 & 38 \\
\hline 33 & (3S)Galb1-4(Fuca1-3)GlcNAc-Sp8 & 8 & 3 & 34 \\
\hline
\end{tabular}




\begin{tabular}{|c|c|c|c|c|}
\hline 197 & Glca1-6Glca1-6Glcb-Sp8 & 8 & 4 & 53 \\
\hline 486 & $\begin{array}{l}\text { Neu5Aca2-3Galb1-3GIcNAcb1-2Mana1-6(GIcNAcb1-4)(Neu5Aca2-3Galb1-3GlcNAcb1-2Mana1-3)Manb1- } \\
\text { 4GlcNAcb1-4GIcNAc-Sp21 }\end{array}$ & 8 & 9 & 111 \\
\hline 39 & $(6 S)(4 S) G a l b 1-4 G I c N A c b-S p 0$ & 8 & 10 & 127 \\
\hline 510 & Galb1-3(6S)GIcNAcb-Sp8 & 8 & 4 & 49 \\
\hline 319 & Galb1-4GIcNAcb1-2Mana1-6(Neu5Aca2-6Galb1-4GIcNAcb1-2Mana1-3)Manb1-4GIcNAcb1-4GIcNAcb-Sp12 & 8 & 10 & 130 \\
\hline 9 & Neu5Aca-Sp8 & 8 & 6 & 74 \\
\hline 581 & $\begin{array}{l}\text { Galb1-4GIcNAcb1-3Galb1-4GIcNAcb1-3Galb1-4GlcNAcb1-3Galb1-4GlcNAcb1-3Galb1-4GIcNAcb1-2Mana1- } \\
\text { 6(Galb1-4GlcNAcb1-3Galb1-4GIcNAcb1-3Galb1-4GIcNAcb1-3Galb1-4GIcNAcb1-3Galb1-4GIcNAcb1-2Mana1- } \\
\text { 3)Manb1-4GIcNAcb1-4(Fuca1-6)GlcNAcb-Sp19 }\end{array}$ & 8 & 10 & 125 \\
\hline 240 & Neu5Aca2-3Galb1-3(Fuca1-4)GlcNAcb1-3Galb1-4(Fuca1-3)GlcNAcb-Sp0 & 8 & 9 & 122 \\
\hline 114 & Gala1-3Galb1-3GlcNAcb-Sp0 & 8 & 4 & 48 \\
\hline 516 & (4S)GalNAcb-Sp10 & 8 & 11 & 149 \\
\hline 326 & $\begin{array}{l}\text { Neu5Aca2-3Galb1-4GIcNAcb1-2Mana1-6(Neu5Aca2-6Galb1-4GlcNAcb1-2Mana1-3)Manb1-4GlcNAcb1- } \\
\text { 4GlcNAcb-Sp12 }\end{array}$ & 8 & 3 & 43 \\
\hline 429 & Galb1-4GIcNAcb1-6(Fuca1-2Galb1-3GIcNAcb1-3)Galb1-4GIc-Sp21 & 8 & 7 & 91 \\
\hline 223 & Neu5Aca2-3Galb1-3GalNAca-Sp8 & 8 & 2 & 25 \\
\hline 167 & Galb1-4GIcNAcb1-6(Galb1-3)GalNAc-Sp14 & 8 & 3 & 43 \\
\hline 331 & Neu5Aca2-3Galb1-3(Fuca1-4)GIcNAcb1-3Galb1-3(Fuca1-4)GIcNAcb-Sp0 & 7 & 6 & 81 \\
\hline 164 & Galb1-4GIcNAcb1-3Galb1-4GIcb-Sp0 & 7 & 3 & 44 \\
\hline 295 & Neu5Aca2-3Galb1-4GIcNAcb1-3Galb1-3GIcNAcb-Sp0 & 7 & 6 & 84 \\
\hline 566 & GlcNAb1-3Galb1-3GalNAc-Sp14 & 7 & 5 & 61 \\
\hline 253 & Neu5Aca2-3Galb1-4(Fuca1-3)GlcNAcb1-3Galb1-4(Fuca1-3)GlcNAcb1-3Galb1-4(Fuca1-3)GIcNAcb-Sp0 & 7 & 6 & 75 \\
\hline 183 & GIcNAcb1-3Galb1-4GIcNAcb-Sp8 & 7 & 7 & 93 \\
\hline 343 & GlcNAca1-4Galb1-4GIcNAcb1-3Galb1-4GIcNAcb-Sp0 & 7 & 8 & 109 \\
\hline 352 & GIcNAcb1-2Mana1-6(GIcNAcb1-2Mana1-3)Manb1-4GIcNAcb1-4(Fuca1-6)GIcNAcb-Sp22 & 7 & 1 & 18 \\
\hline 459 & $\begin{array}{l}\text { Neu5Aca2-3Galb1-4GIcNAcb1-2Mana1-6(GIcNAcb1-4)(Neu5Aca2-3Galb1-4GlcNAcb1-2Mana1-3)Manb1- } \\
\text { 4GlcNAcb1-4GIcNAcb-Sp21 }\end{array}$ & 7 & 8 & 112 \\
\hline 453 & GalNAcb1-4Galb1-4GIcb-Sp0 & 7 & 8 & 105 \\
\hline 106 & Gala1-3(Fuca1-2)Galb1-4Glcb-Sp0 & 7 & 9 & 120 \\
\hline 455 & $\begin{array}{l}\text { Gala1-3(Fuca1-2)Galb1-3GlcNAcb1-2Mana1-6(Gala1-3(Fuca1-2)Galb1-3GlcNAcb1-2Mana1-3)Manb1- } \\
\text { 4GlcNAcb1-4(Fuca1-6)GlcNAcb-Sp22 }\end{array}$ & 7 & 3 & 41 \\
\hline 75 & Fuca1-2Galb1-4GIcNAcb-Sp0 & 7 & 5 & 75 \\
\hline 259 & Neu5Aca2-3Galb1-4GlcNAcb-Sp0 & 7 & 1 & 18 \\
\hline 402 & GalNAca1-3GalNAcb1-3Gala1-4Galb1-4GIcNAcb-Sp0 & 7 & 5 & 71 \\
\hline 483 & $\begin{array}{l}\text { Neu5Aca2-3Galb1-4GIcNAcb1-2Mana1-6(Neu5Aca2-3Galb1-4GlcNAcb1-2Mana1-3)Manb1-4GIcNAcb1-4(Fuca1- } \\
\text { 6)GIcNAcb-Sp24 }\end{array}$ & 7 & 3 & 39 \\
\hline 398 & Galb1-4GIcNAcb1-2Mana1-6(GIcNAcb1-2Mana1-3)Manb1-4GIcNAcb1-4GIcNAc-Sp12 & 7 & 11 & 151 \\
\hline 262 & Fuca1-2Galb1-4(6S)GIcb-Sp0 & 7 & 3 & 41 \\
\hline 47 & (6S)GIcNAcb-Sp8 & 7 & 7 & 97 \\
\hline 151 & Galb1-4(Fuca1-3)GlcNAcb-Sp0 & 7 & 3 & 46 \\
\hline 556 & Neu5Gca2-8Neu5Gca2-6Galb1-4GlcNAc-Sp0 & 7 & 5 & 68 \\
\hline 152 & Galb1-4(Fuca1-3)GlcNAcb-Sp8 & 7 & 4 & 50 \\
\hline
\end{tabular}




\begin{tabular}{|c|c|c|c|c|}
\hline 7 & Fuca-Sp9 & 7 & 5 & 67 \\
\hline 309 & GlcNAcb1-3Man-Sp10 & 7 & 6 & 81 \\
\hline 174 & GlcNAca1-6Galb1-4GlcNAcb-Sp8 & 7 & 0 & 6 \\
\hline 549 & $\begin{array}{l}\text { GlcNAcb1-3Galb1-4GlcNAcb1-3Galb1-4GIcNAcb1-3Galb1-4GlcNAcb1-2Mana1-6(GlcNAcb1-3Galb1-4GlcNAcb1- } \\
\text { 3Galb1-4GIcNAcb1-3Galb1-4GIcNAcb1-2Mana1-3)Manb1-4GIcNAcb1-4GIcNAcb-Sp25 }\end{array}$ & 7 & 3 & 39 \\
\hline 348 & Neu5Aca2-6Galb1-4GIcNAcb1-2Mana1-3Manb1-4GIcNAcb1-4GIcNAc-Sp12 & 7 & 3 & 44 \\
\hline 401 & Galb1-4(Fuca1-3)GlcNAcb1-3GalNAca-Sp14 & 7 & 6 & 92 \\
\hline 404 & Gala1-4Galb1-4GIcNAcb1-2Mana1-6(Gala1-4Galb1-4GIcNAcb1-2Mana1-3)Manb1-4GIcNAcb1-4GIcNAcb-Sp24 & 7 & 8 & 110 \\
\hline 179 & GlcNAcb1-3GalNAca-Sp8 & 7 & 6 & 88 \\
\hline 15 & GalNAcb-Sp8 & 7 & 8 & 122 \\
\hline 166 & Galb1-4GIcNAcb1-6(Galb1-3)GalNAca-Sp8 & 7 & 4 & 65 \\
\hline 201 & GlcAa-Sp8 & 7 & 6 & 95 \\
\hline 577 & $\begin{array}{l}\text { Galb1-4GIcNAcb1-3Galb1-4GIcNAcb1-3Galb1-4GIcNAcb1-2Mana1-6(Galb1-4GIcNAcb1-3Galb1-4GIcNAcb1- } \\
\text { 3Galb1-4GIcNAcb1-2Mana1-3)Manb1-4GIcNAcb1-4(Fuca1-6)GIcNAcb-Sp24 }\end{array}$ & 7 & 9 & 126 \\
\hline 246 & Neu5Aca2-3Galb1-3GalNAcb1-3Gala1-4Galb1-4GIcb-Sp0 & 7 & 6 & 96 \\
\hline 388 & GlcNAcb1-2Mana1-6(GlcNAcb1-4(GlcNAcb1-2)Mana1-3)Manb1-4GlcNAcb1-4GIcNAc-Sp21 & 7 & 4 & 62 \\
\hline 171 & Galb1-4Glcb-Sp0 & 7 & 5 & 76 \\
\hline 269 & Neu5Aca2-6Galb1-4GIcNAcb-Sp8 & 7 & 2 & 28 \\
\hline 541 & $\begin{array}{l}\text { Galb1-4GIcNAcb1-3Galb1-4GIcNAcb1-2Mana1-6(Galb1-4GlcNAcb1-3Galb1-4GlcNAcb1-2Mana1-3)Manb1- } \\
\text { 4GlcNAcb1-4GIcNAcb-Sp12 }\end{array}$ & 7 & 4 & 61 \\
\hline 480 & Neu5Aca2-6Galb1-4GIcNAcb1-6GalNAca-Sp14 & 7 & 5 & 70 \\
\hline 563 & GalNAcb1-4GIcNAcb1-3GalNAcb1-4GIcNAcb-Sp0 & 7 & 2 & 30 \\
\hline 466 & $\begin{array}{l}\text { Neu5Aca2-6Galb1-4GlcNAcb1-6(Neu5Aca2-6Galb1-4GlcNAcb1-2)Mana1-6(GlcNAcb1-4)(Neu5Aca2-6Galb1- } \\
\text { 4GIcNAcb1-4(Neu5Aca2-6Galb1-4GIcNAcb1-2)Mana1-3)Manb1-4GIcNAcb1-4GIcNAcb-Sp21 }\end{array}$ & 7 & 3 & 39 \\
\hline 237 & Neu5Aca2-3GalNAcb1-4GIcNAcb-Sp0 & 7 & 2 & 34 \\
\hline 400 & Fuca1-2Galb1-4GlcNAcb1-3GalNAca-Sp14 & 7 & 10 & 145 \\
\hline 170 & Galb1-4GlcNAcb-Sp23 & 7 & 5 & 78 \\
\hline 32 & (3S)Galb1-4(Fuca1-3)GlcNAc-Sp0 & 7 & 4 & 68 \\
\hline 368 & Galb1-4(Fuca1-3)GlcNAcb1-6(Fuca1-2Galb1-4GIcNAcb1-3)Galb1-4Glc-Sp21 & 6 & 1 & 22 \\
\hline 371 & $\begin{array}{l}\text { Gala1-3(Fuca1-2)Galb1-4GlcNAcb1-2Mana1-6(Gala1-3(Fuca1-2)Galb1-4GIcNAcb1-2Mana1-3)Manb1- } \\
\text { 4GIcNAcb1-4GIcNAcb-Sp20 }\end{array}$ & 6 & 4 & 57 \\
\hline 129 & Galb1-3(Fuca1-4)GlcNAc-Sp8 & 6 & 5 & 71 \\
\hline 379 & GalNAcb1-4GIcNAcb1-2Mana1-6(GalNAcb1-4GIcNAcb1-2Mana1-3)Manb1-4GIcNAcb1-4GIcNAc-Sp12 & 6 & 2 & 33 \\
\hline 12 & Galb-Sp8 & 6 & 6 & 94 \\
\hline 176 & GlcNAcb1-6(GlcNAcb1-3)GalNAca-Sp8 & 6 & 2 & 38 \\
\hline 411 & Galb1-3GalNAcb1-4(Neu5Aca2-8Neu5Aca2-3)Galb1-4Glcb-Sp0 & 6 & 3 & 47 \\
\hline 608 & $\begin{array}{l}\text { Neu5Aca2-6Galb1-4GlcNAcb1-3Galb1-4GlcNAcb1-2Mana1-6(Neu5Aca2-6Galb1-4GlcNAcb1-3Galb1- } \\
\text { 4GlcNAcb1-2Mana1-3)Manb1-4GlcNAcb1-4GlcNAcb-Sp12 }\end{array}$ & 6 & 6 & 90 \\
\hline 300 & Galb1-3Galb1-4GlcNAcb-Sp8 & 6 & 9 & 146 \\
\hline 115 & Gala1-3Galb1-4GIcNAcb-Sp8 & 6 & 1 & 18 \\
\hline 456 & Neu5Aca2-6Galb1-4GIcNAcb1-6(Fuca1-2Galb1-3GIcNAcb1-3)Galb1-4GIc-Sp21 & 6 & 6 & 89 \\
\hline 321 & Neu5Aca2-8Neu5Acb-Sp17 & 6 & 4 & 61 \\
\hline 159 & Galb1-4GIcNAcb1-3GalNAca-Sp8 & 6 & 3 & 52 \\
\hline
\end{tabular}




\begin{tabular}{|c|c|c|c|c|}
\hline 415 & GalNAca1-3GalNAcb1-3Gala1-4Galb1-4Glcb-Sp0 & 6 & 3 & 51 \\
\hline 390 & Fuca1-2Galb1-3GalNAca1-3(Fuca1-2)Galb1-4GIcNAcb-Sp0 & 6 & 8 & 123 \\
\hline 328 & Neu5,9Ac2a2-3Galb1-4GIcNAcb-Sp0 & 6 & 4 & 62 \\
\hline 92 & GalNAca1-3Galb-Sp8 & 6 & 5 & 84 \\
\hline 522 & Gala1-3(Fuca1-2)Galb1-4GIcNAcb1-2Mana-Sp0 & 6 & 4 & 65 \\
\hline 217 & Manb1-4GIcNAcb-Sp0 & 6 & 4 & 73 \\
\hline 263 & Neu5Aca2-3Galb1-4GIcb-Sp0 & 6 & 2 & 40 \\
\hline 363 & Gala1-3Galb1-4GIcNAcb1-2Mana1-6(Gala1-3Galb1-4GlcNAcb1-2Mana1-3)Manb1-4GlcNAcb1-4GlcNAcb-Sp20 & 6 & 4 & 74 \\
\hline 279 & Neu5Acb2-6Galb1-4GIcNAcb-Sp8 & 6 & 4 & 71 \\
\hline 191 & GlcNAcb1-4GlcNAcb1-4GlcNAcb-Sp8 & 6 & 6 & 100 \\
\hline 384 & Galb1-4GIcNAcb1-6(Fuca1-4(Fuca1-2Galb1-3)GlcNAcb1-3)Galb1-4GIc-Sp21 & 6 & 6 & 96 \\
\hline 421 & GlcNAcb1-2(GlcNAcb1-6)Mana1-6(GlcNAcb1-2Mana1-3)Manb1-4GlcNAcb1-4GlcNAcb-Sp19 & 6 & 1 & 19 \\
\hline 180 & GlcNAcb1-3GalNAca-Sp14 & 6 & 4 & 66 \\
\hline 148 & Galb1-3GlcNAcb1-3Galb1-4Glcb-Sp10 & 6 & 4 & 69 \\
\hline 367 & Neu5Aca2-6GlcNAcb1-4GIcNAcb1-4GlcNAc-Sp21 & 6 & 10 & 166 \\
\hline 461 & $\begin{array}{l}\text { Neu5Aca2-3Galb1-4GIcNAcb1-6(Neu5Aca2-3Galb1-4GIcNAcb1-2)Mana1-6(GlcNAcb1-4)(Neu5Aca2-3Galb1- } \\
\text { 4GIcNAcb1-2Mana1-3)Manb1-4GIcNAcb1-4GIcNAcb-Sp21 }\end{array}$ & 6 & 8 & 144 \\
\hline 145 & Galb1-3GalNAcb1-4Galb1-4Glcb-Sp8 & 6 & 7 & 110 \\
\hline 127 & Galb1-3GlcNAcb1-3Galb1-4(Fuca1-3)GlcNAcb-Sp0 & 6 & 5 & 88 \\
\hline 414 & GalNAca1-3(Fuca1-2)Galb1-4GIcNAcb1-3GalNAca-Sp14 & 6 & 3 & 43 \\
\hline 532 & $\begin{array}{l}\text { Galb1-4GIcNAcb1-2 Mana1-6(Galb1-4GlcNAcb1-4)(Galb1-4GIcNAcb1-2Mana1-3)Manb1-4GIcNAcb1-4(Fuca1- } \\
\text { 6)GIcNAc-Sp21 }\end{array}$ & 6 & 3 & 58 \\
\hline 428 & $\begin{array}{l}\text { Galb1-3GlcNAcb1-6(Galb1-3GIcNAcb1-2)Mana1-6(Galb1-3GIcNAcb1-2Mana1-3)Manb1-4GIcNAcb1-4GlcNAcb- } \\
\text { Sp19 }\end{array}$ & 6 & 3 & 46 \\
\hline 443 & (6S)Galb1-3GlcNAcb-Sp0 & 6 & 5 & 95 \\
\hline 370 & $\begin{array}{l}\text { GalNAca1-3(Fuca1-2)Galb1-4GlcNAcb1-2Mana1-6(GalNAca1-3(Fuca1-2)Galb1-4GlcNAcb1-2Mana1-3)Manb1- } \\
\text { 4GlcNAcb1-4GlcNAcb-Sp20 }\end{array}$ & 6 & 6 & 110 \\
\hline 392 & GalNAcb1-4(Neu5Aca2-3)Galb1-4GIcNAcb1-3GalNAca-Sp14 & 6 & 3 & 48 \\
\hline 118 & Gala1-3Galb-Sp8 & 6 & 6 & 98 \\
\hline 260 & Neu5Aca2-3Galb1-4GIcNAcb-Sp8 & 6 & 1 & 25 \\
\hline 3 & Mana-Sp8 & 6 & 11 & 196 \\
\hline 45 & (6S)Galb1-4(6S)Glcb-Sp8 & 6 & 4 & 74 \\
\hline 265 & Neu5Aca2-6GalNAca-Sp8 & 6 & 2 & 38 \\
\hline 340 & GIcNAca1-4Galb1-3GIcNAcb-Sp0 & 6 & 8 & 141 \\
\hline 221 & Fuca1-2Galb1-4(6S)GlcNAcb-Sp8 & 6 & 2 & 41 \\
\hline 268 & Neu5Aca2-6Galb1-4GIcNAcb-Sp0 & 6 & 6 & 98 \\
\hline 568 & $\begin{array}{l}\text { Galb1-4GIcNAcb1-3Galb1-4GIcNAcb1-3Galb1-4GlcNAcb1-3Galb1-4GIcNAcb1-3Galb1-4GIcNAcb1-3Galb1- } \\
\text { 4GlcNAcb1-2Mana1-6(Galb1-4GıNAcb1-3Galb1-4GIcNAcb1-3Galb1-4GlcNAcb1-3Galb1-4GIcNAcb1-3Galb1- } \\
\text { 4GIcNAcb1-3Galb1-4GlcNAcb1-2Mana1-3)Manb1-4GIcNAcb1-4GIcNAcb-Sp25 }\end{array}$ & 6 & 5 & 83 \\
\hline 161 & Galb1-4GIcNAcb1-3Galb1-4(Fuca1-3)GlcNAcb1-3Galb1-4(Fuca1-3)GIcNAcb-Sp0 & 6 & 8 & 144 \\
\hline 5 & GalNAca-Sp15 & 6 & 4 & 66 \\
\hline 277 & Galb1-3(Fuca1-4)GlcNAcb1-3Galb1-3(Fuca1-4)GlcNAcb-Sp0 & 6 & 4 & 69 \\
\hline 44 & (6S)Galb1-4GlcNAcb-Sp8 & 6 & 0 & 9 \\
\hline
\end{tabular}




\begin{tabular}{|c|c|c|c|c|}
\hline 251 & Neu5Aca2-3Galb1-4(6S)GlcNAcb-Sp8 & 6 & 9 & 154 \\
\hline 603 & $\begin{array}{l}\text { Neu5Aca2-3Galb1-4GIcNAcb1-3Galb1-4GIcNAcb1-2Mana1-6(Neu5Aca2-3Galb1-4GlcNAcb1-3Galb1- } \\
\text { 4GIcNAcb1-2Mana1-3)Manb1-4GlcNAcb1-4GIcNAcb-Sp12 }\end{array}$ & 6 & 6 & 107 \\
\hline 302 & Galb1-4GlcNAcb1-6(Galb1-4GIcNAcb1-3)Galb1-4GlcNAc-Sp0 & 6 & 5 & 96 \\
\hline 609 & GlcNAcb1-3Fuca-Sp21 & 6 & 6 & 116 \\
\hline 601 & Neu5Aca2-6Galb1-4GIcNAcb1-3Galb1-4GlcNAcb1-6(Galb1-3)GalNAca-Sp14 & 6 & 5 & 82 \\
\hline 205 & KDNa2-3Galb1-3GlcNAcb-Sp0 & 6 & 6 & 101 \\
\hline 188 & GlcNAcb1-4Galb1-4GlcNAcb-Sp8 & 6 & 4 & 74 \\
\hline 550 & $\begin{array}{l}\text { Galb1-4GlcNAcb1-3Galb1-4GlcNAcb1-3Galb1-4GlcNAcb1-3Galb1-4GlcNAcb1-2Mana1-6(Galb1-4GlcNAcb1- } \\
\text { 3Galb1-4GlcNAcb1-3Galb1-4GlcNAcb1-3Galb1-4GIcNAcb1-2Mana1-3)Manb1-4GlcNAcb1-4GlcNAcb-Sp25 }\end{array}$ & 6 & 7 & 122 \\
\hline 307 & GlcAb1-3GIcNAcb-Sp8 & 5 & 8 & 144 \\
\hline 136 & Neu5Aca2-6(Galb1-3)GalNAca-Sp14 & 5 & 2 & 45 \\
\hline 233 & GalNAcb1-4(Neu5Aca2-3)Galb1-4Glcb-Sp0 & 5 & 5 & 88 \\
\hline 434 & $\begin{array}{l}\text { GIcNAcb1-6(GlcNAcb1-2)Mana1-6(GlcNAcb1-4)(GlcNAcb1-4(GIcNAcb1-2)Mana1-3)Manb1-4GlcNAcb1- } \\
\text { 4GIcNAc-Sp21 }\end{array}$ & 5 & 5 & 92 \\
\hline 536 & GalNAca1-3(Fuca1-2)Galb1-3GalNAcb1-3Gala1-4Galb1-4Glc-Sp21 & 5 & 9 & 159 \\
\hline 559 & $\begin{array}{l}\text { Galb1-4GIcNAcb1-3Galb1-4GlcNAcb1-6(Galb1-4GIcNAcb1-3Galb1-4GlcNAcb1-2)Mana1-6(Galb1-4GlcNAcb1- } \\
\text { 3Galb1-4GlcNAcb1-2Mana1-3)Mana1-4GIcNAcb1-4GIcNAc-Sp24 }\end{array}$ & 5 & 4 & 66 \\
\hline 449 & Fuca1-2Galb1-4GIcNAcb1-6(Fuca1-2Galb1-4GIcNAcb1-3)GalNAc-Sp14 & 5 & 6 & 111 \\
\hline 310 & GlcNAcb1-4GlcNAcb-Sp10 & 5 & 7 & 127 \\
\hline 157 & Galb1-4GalNAca1-3(Fuca1-2)Galb1-4GIcNAcb-Sp8 & 5 & 8 & 148 \\
\hline 264 & Neu5Aca2-3Galb1-4Glcb-Sp8 & 5 & 5 & 87 \\
\hline 149 & Galb1-3GlcNAcb-Sp0 & 5 & 6 & 114 \\
\hline 359 & KDNa2-3Galb1-3GalNAca-Sp14 & 5 & 8 & 154 \\
\hline 468 & Gala1-3(Fuca1-2)Galb1-3GalNAcb-Sp8 & 5 & 7 & 137 \\
\hline 445 & $\begin{array}{l}\text { Fuca1-2Galb1-4 GlcNAcb1-2Mana1-6(Fuca1-2Galb1-4GlcNAcb1-2(Fuca1-2Galb1-4GIcNAcb1-4)Mana1- } \\
\text { 3)Manb1-4GlcNAcb1-4GlcNAcb-Sp12 }\end{array}$ & 5 & 2 & 30 \\
\hline 320 & GlcNAcb1-2Mana1-6(Neu5Aca2-6Galb1-4GIcNAcb1-2Mana1-3)Manb1-4GIcNAcb1-4GIcNAcb-Sp12 & 5 & 4 & 70 \\
\hline 282 & Neu5Gca2-3Galb1-4(Fuca1-3)GlcNAcb-Sp0 & 5 & 4 & 79 \\
\hline 540 & $\begin{array}{l}\text { GlcNAcb1-3Galb1-4GIcNAcb1-2Mana1-6(GlcNAcb1-3Galb1-4GIcNAcb1-2Mana1-3)Manb1-4GIcNAcb1- } \\
\text { 4GIcNAcb-Sp25 }\end{array}$ & 5 & 2 & 47 \\
\hline 13 & Glcb-Sp8 & 5 & 2 & 42 \\
\hline 123 & Gala1-4GIcNAcb-Sp8 & 5 & 5 & 99 \\
\hline 517 & Galb1-4(6P)GlcNAcb-Sp0 & 5 & 6 & 126 \\
\hline 293 & Galb1-4GlcNAcb1-3Galb1-3GlcNAcb-Sp0 & 5 & 2 & 32 \\
\hline 153 & Galb1-4(Fuca1-3)GlcNAcb1-3Galb1-4(Fuca1-3)GlcNAcb-Sp0 & 5 & 3 & 52 \\
\hline 274 & Neu5Aca2-6Galb-Sp8 & 5 & 5 & 93 \\
\hline 291 & Galb1-4(Fuca1-3)(6S)Glcb-Sp0 & 5 & 7 & 136 \\
\hline 24 & (3S)Galb1-4(Fuca1-3)(6S)GIc-Sp0 & 5 & 5 & 106 \\
\hline 184 & GlcNAcb1-3Galb1-4GIcNAcb1-3Galb1-4GIcNAcb-Sp0 & 5 & 3 & 50 \\
\hline 376 & Neu5Aca2-3Galb1-4GIcNAcb1-3GalNAc-Sp14 & 5 & 2 & 44 \\
\hline
\end{tabular}




\begin{tabular}{|c|c|c|c|c|}
\hline 281 & Neu5Gca2-3Galb1-3GlcNAcb-Sp0 & 5 & 3 & 55 \\
\hline 60 & Fuca1-2Galb1-3(Fuca1-4)GIcNAcb-Sp8 & 5 & 4 & 84 \\
\hline 218 & Neu5Aca2-3Galb1-4GIcNAcb1-3Galb1-4(Fuca1-3)GlcNAcb-Sp0 & 5 & 2 & 48 \\
\hline 534 & Neu5Aca2-3Galb1-4(Fuca1-3)GlcNAcb1-2Mana-Sp0 & 5 & 4 & 77 \\
\hline 67 & Fuca1-2Galb1-3GlcNAcb-Sp0 & 5 & 7 & 136 \\
\hline 6 & Fuca-Sp8 & 5 & 8 & 160 \\
\hline 168 & Galb1-4GlcNAcb-Sp0 & 5 & 4 & 83 \\
\hline 160 & Galb1-4GIcNAcb1-3GalNAc-Sp14 & 5 & 4 & 88 \\
\hline 210 & Mana1-6(Mana1-2Mana1-3)Mana1-6(Mana1-2Mana1-3)Manb1-4GIcNAcb1-4GIcNAcb-Sp12 & 5 & 9 & 181 \\
\hline 589 & Galb1-4GIcNAcb1-3Galb1-4GIcNAcb1-3GalNAca-Sp14 & 5 & 2 & 43 \\
\hline 381 & Galb1-3GalNAca1-3(Fuca1-2)Galb1-4GIcNAc-Sp0 & 5 & 4 & 73 \\
\hline 476 & Galb1-3GIcNAcb1-2Mana1-6(GlcNAcb1-4)(Galb1-3GIcNAcb1-2Mana1-3)Manb1-4GIcNAcb1-4GIcNAcb-Sp21 & 5 & 6 & 125 \\
\hline 147 & Galb1-3GIcNAcb1-3Galb1-4GIcNAcb-Sp0 & 5 & 4 & 83 \\
\hline 533 & Fuca1-4(Galb1-3)GIcNAcb1-2 Mana-Sp0 & 5 & 3 & 57 \\
\hline 504 & (3S)GalNAcb1-4(Fuca1-3)GlcNAcb-Sp8 & 5 & 5 & 108 \\
\hline 538 & Galb1-3GalNAcb1-3Gal-Sp21 & 5 & 5 & 115 \\
\hline 301 & Neu5Aca2-6Galb1-4GIcNAcb1-2Mana1-6(Galb1-4GIcNAcb1-2Mana1-3)Manb1-4GIcNAcb1-4GIcNAcb-Sp12 & 5 & 7 & 151 \\
\hline 245 & Neu5Aca2-3Galb-Sp8 & 5 & 6 & 121 \\
\hline 103 & Gala1-3(Fuca1-2)Galb1-4(Fuca1-3)GlcNAcb-Sp0 & 5 & 5 & 108 \\
\hline 493 & Galb1-4(Fuca1-3)GlcNAcb1-6(Neu5Aca2-6(Neu5Aca2-3Galb1-3)GlcNAcb1-3)Galb1-4Glc-Sp21 & 5 & 6 & 129 \\
\hline 231 & GalNAcb1-4(Neu5Aca2-3)Galb1-4GIcNAcb-Sp0 & 5 & 3 & 61 \\
\hline 596 & $\begin{array}{l}\text { Neu5Aca2-3Galb1-4GIcNAcb1-3Galb1-4GIcNAcb1-6(Neu5Aca2-3Galb1-4GIcNAcb1-3Galb1-4GIcNAcb1- } \\
\text { 3)GalNAca-Sp14 }\end{array}$ & 5 & 4 & 93 \\
\hline 318 & $\begin{array}{l}\text { Neu5Aca2-6Galb1-4GIcNAcb1-2Mana1-6(Neu5Aca2-3Galb1-4GIcNAcb1-2Mana1-3)Manb1-4GIcNAcb1- } \\
\text { 4GIcNAcb-Sp12 }\end{array}$ & 5 & 8 & 171 \\
\hline 140 & Galb1-3GalNAca-Sp14 & 5 & 5 & 106 \\
\hline 58 & Fuca1-2Galb1-3GalNAcb1-3Gala-Sp9 & 5 & 6 & 127 \\
\hline 194 & GIcNAcb1-6Galb1-4GIcNAcb-Sp8 & 5 & 4 & 88 \\
\hline 292 & Galb1-4(Fuca1-3)GlcNAcb1-3Galb1-3(Fuca1-4)GlcNAcb-Sp0 & 4 & 7 & 154 \\
\hline 451 & GalNAca1-3(Fuca1-2)Galb1-4GIcNAcb1-6(GalNAca1-3(Fuca1-2)Galb1-4GIcNAcb1-3)GalNAc-Sp14 & 4 & 3 & 65 \\
\hline 471 & Neu5Aca2-3Galb1-4GIcNAcb1-6(Neu5Aca2-3Galb1-4GIcNAcb1-3)GalNAca-Sp14 & 4 & 3 & 77 \\
\hline 409 & Gala1-3(Fuca1-2)Galb1-4(Fuca1-3)Glcb-Sp21 & 4 & 3 & 66 \\
\hline 508 & $\begin{array}{l}\text { Galb1-4GIcNAcb1-6(Galb1-4GlcNAcb1-2)Mana1-6(GlcNAcb1-4)Galb1-4GlcNAcb1-4(Gal b1-4GIcNAcb1- } \\
\text { 2)Mana1-3)Manb1-4GIcNAcb1-4(Fuca1-6)GlcNAc-Sp21 }\end{array}$ & 4 & 5 & 112 \\
\hline 273 & Neu5Aca2-6Galb1-4GIcb-Sp8 & 4 & 3 & 61 \\
\hline 112 & Gala1-3GalNAcb-Sp8 & 4 & 6 & 131 \\
\hline 125 & Galb1-2Galb-Sp8 & 4 & 6 & 141 \\
\hline 497 & Fuca1-2(6S)Galb1-3GIcNAcb-Sp0 & 4 & 5 & 105 \\
\hline
\end{tabular}




\begin{tabular}{|c|c|c|c|c|}
\hline 54 & Galb1-4GIcNAcb1-2Mana1-6(Galb1-4GIcNAcb1-2Mana1-3)Manb1-4GIcNAcb1-4GIcNAcb-Sp12 & 4 & 4 & 89 \\
\hline 78 & Fuca1-2Galb-Sp8 & 4 & 2 & 50 \\
\hline 137 & Neu5Acb2-6(Galb1-3)GalNAca-Sp8 & 4 & 7 & 156 \\
\hline 270 & Neu5Aca2-6Galb1-4GIcNAcb1-3Galb1-4(Fuca1-3)GIcNAcb1-3Galb1-4(Fuca1-3)GlcNAcb-Sp0 & 4 & 3 & 78 \\
\hline 206 & KDNa2-3Galb1-4GIcNAcb-Sp0 & 4 & 4 & 102 \\
\hline 335 & GalNAca1-3(Fuca1-2)Galb1-4GIcNAcb1-3Galb1-4GlcNAcb-Sp0 & 4 & 3 & 64 \\
\hline 438 & $\begin{array}{l}\text { Galb1-4GIcNAcb1-6(Galb1-4GIcNAcb1-2)Mana1-6(GlcNAcb1-4)(Galb1-4GlcNAcb1-4(Galb1-4GlcNAcb1- } \\
\text { 2)Mana1-3)Manb1-4GIcNAcb1-4GIcNAc-Sp21 }\end{array}$ & 4 & 2 & 58 \\
\hline 439 & Galb1-4Galb-Sp10 & 4 & 6 & 146 \\
\hline 463 & $\begin{array}{l}\text { Neu5Aca2-6Galb1-4GIcNAcb1-2Mana1-6(GIcNAcb1-4)(Neu5Aca2-6Galb1-4GlcNAcb1-2Mana1-3)Manb1- } \\
\text { 4GIcNAcb1-4GlcNAcb-Sp21 }\end{array}$ & 4 & 4 & 89 \\
\hline 324 & Galb1-3GlcNAcb1-2Mana1-6(Galb1-3GIcNAcb1-2Mana1-3)Manb1-4GIcNAcb1-4GIcNAcb-Sp19 & 4 & 4 & 102 \\
\hline 57 & $\begin{array}{l}\text { Neu5Aca2-6Galb1-4GIcNAcb1-2Mana1-6(Neu5Aca2-6Galb1-4GIcNAcb1-2Mana1-3)Manb1-4GlcNAcb1- } \\
\text { 4GIcNAcb-Sp24 }\end{array}$ & 4 & 2 & 54 \\
\hline 602 & Neu5Aca2-6Galb1-4GIcNAcb1-6(Galb1-3)GalNAca-Sp14 & 4 & 6 & 147 \\
\hline 98 & GalNAcb1-4GIcNAcb-Sp0 & 4 & 3 & 83 \\
\hline 496 & Galb1-4(Fuca1-3)GIcNAcb1-2Mana-Sp0 & 4 & 5 & 124 \\
\hline 494 & Fuca1-2Galb1-4GlcNAcb1-6GalNAca-Sp14 & 4 & 7 & 177 \\
\hline 85 & GaINAca1-3(Fuca1-2)Galb1-4GlcNAcb-Sp0 & 4 & 5 & 117 \\
\hline 525 & Gala1-3(Fuca1-2)Galb1-3GlcNAcb1-6GalNAc-Sp14 & 4 & 3 & 84 \\
\hline 505 & Fuca1-2Galb1-3GlcNAcb1-6(Fuca1-2Galb1-3GlcNAcb1-3)GalNAca-Sp14 & 4 & 7 & 177 \\
\hline 397 & GlcNAcb1-2Mana1-6(Galb1-4GIcNAcb1-2Mana1-3)Manb1-4GIcNAcb1-4GIcNAc-Sp12 & 4 & 4 & 93 \\
\hline 234 & Neu5Aca2-3Galb1-3GalNAcb1-4(Neu5Aca2-3)Galb1-4Glcb-Sp0 & 4 & 2 & 50 \\
\hline 345 & Neu5Aca2-6Galb1-4GIcNAcb1-2Mana1-6(Mana1-3)Manb1-4GIcNAcb1-4GIcNAc-Sp12 & 4 & 1 & 26 \\
\hline 227 & Neu5Aca2-8Neu5Aca2-8Neu5Aca2-3Galb1-4Glcb-Sp0 & 4 & 2 & 44 \\
\hline 43 & (6S)Galb1-4Glcb-Sp8 & 4 & 4 & 93 \\
\hline 349 & Galb1-4GIcNAcb1-2Mana1-3Manb1-4GIcNAcb1-4GIcNAc-Sp12 & 4 & 4 & 104 \\
\hline 10 & Neu5Aca-Sp11 & 4 & 5 & 132 \\
\hline 490 & Galb1-3(Fuca1-4)GlcNAcb1-6GalNAca-Sp14 & 4 & 10 & 270 \\
\hline 470 & Glca1-4GIca1-4GIca1-4GIcb-Sp10 & 4 & 10 & 250 \\
\hline 229 & Neu5Aca2-8Neu5Aca2-8Neu5Aca-Sp8 & 4 & 5 & 124 \\
\hline 524 & Galb1-3GIcNAcb1-2Mana-Sp0 & 4 & 6 & 159 \\
\hline 452 & Neu5Aca2-8Neu5Aca2-3Galb1-3GalNAcb1-4(Neu5Aca2-8Neu5Aca2-3)Galb1-4GIcb-Sp0 & 4 & 5 & 142 \\
\hline 135 & Neu5Aca2-6(Galb1-3)GalNAca-Sp8 & 4 & 4 & 107 \\
\hline 102 & Gala1-3(Fuca1-2)Galb1-3GIcNAcb-Sp8 & 4 & 3 & 74 \\
\hline 213 & Mana1-6(Mana1-3)Mana-Sp9 & 4 & 6 & 164 \\
\hline 406 & Galb1-3GlcNAcb1-6Galb1-4GIcNAcb-Sp0 & 4 & 3 & 78 \\
\hline 571 & $\begin{array}{l}\text { Galb1-3GlcNAcb1-3Galb1-4GlcNAcb1-3Galb1-4GlcNAcb1-6(Galb1-3GlcNAcb1-3Galb1-4GlcNAcb1-3Galb1- } \\
\text { 4GlcNAb1-2)Mana1-6(Galb1-3GlcNAcb1-3Galb1-4GIcNAcb1-3Galb1-4GlcNAcb1-2Mana1-3)Manb1-4GIcNAcb1- } \\
\text { 4(Fuca1-6)GlcNAcb-Sp24 }\end{array}$ & 4 & 3 & 77 \\
\hline
\end{tabular}




\begin{tabular}{|c|c|c|c|c|}
\hline 561 & GlcNAcb1-3Galb1-4GlcNAcb1-6(GlcNAcb1-3Galb1-3)GalNAca-Sp14 & 4 & 9 & 245 \\
\hline 299 & Neu5Aca2-3Galb1-4(Fuca1-3)GIcNAcb1-6(Galb1-3)GalNAca-Sp14 & 4 & 4 & 122 \\
\hline 267 & Neu5Aca2-6Galb1-4(6S)GlcNAcb-Sp8 & 4 & 3 & 94 \\
\hline 548 & $\begin{array}{l}\text { Galb1-4GIcNAcb1-3Galb1-4GlcNAcb1-3Galb1-4GlcNAcb1-2Mana1-6(Galb1-4GlcNAcb1-3Galb1-4GIcNAcb1- } \\
\text { 3Galb1-4GlcNAcb1-2Mana1-3)Manb1-4GlcNAcb1-4GlcNAcb-Sp24 }\end{array}$ & 4 & 3 & 74 \\
\hline 385 & Galb1-4(Fuca1-3)GIcNAcb1-6(Fuca1-4(Fuca1-2Galb1-3)GIcNAcb1-3)Galb1-4GIc-Sp21 & 4 & 4 & 101 \\
\hline 426 & $\begin{array}{l}\text { Fuca1-2Galb1-3GIcNAcb1-2Mana1-6(Fuca1-2Galb1-3GIcNAcb1-2Mana1-3)Manb1-4GIcNAcb1-4(Fuca1- } \\
\text { 6)GIcNAcb-Sp22 }\end{array}$ & 4 & 3 & 85 \\
\hline 386 & Galb1-3GIcNAcb1-3Galb1-4(Fuca1-3)GIcNAcb1-6(Galb1-3GIcNAcb1-3)Galb1-4GIc-Sp21 & 4 & 5 & 137 \\
\hline 232 & GalNAcb1-4(Neu5Aca2-3)Galb1-4GlcNAcb-Sp8 & 4 & 3 & 98 \\
\hline 63 & Fuca1-2Galb1-3GalNAcb1-4(Neu5Aca2-3)Galb1-4Glcb-Sp0 & 4 & 4 & 105 \\
\hline 193 & GlcNAcb1-6GalNAca-Sp14 & 3 & 7 & 193 \\
\hline 521 & Gala1-3Galb1-4GlcNAcb1-2Mana-Sp0 & 3 & 5 & 134 \\
\hline 457 & $\begin{array}{l}\text { GalNAca1-3(Fuca1-2)Galb1-3GlcNAcb1-2Mana1-6(GalNAca1-3(Fuca1-2)Galb1-3GlcNAcb1-2Mana1-3)Manb1- } \\
\text { 4GIcNAcb1-4(Fuca1-6)GIcNAcb-Sp22 }\end{array}$ & 3 & 2 & 55 \\
\hline 423 & Gala1-3(Fuca1-2)Galb1-3GlcNAcb1-3GalNAc-Sp14 & 3 & 4 & 131 \\
\hline 498 & Gala1-3(Fuca1-2)Galb1-4GIcNAcb1-6GaINAca-Sp14 & 3 & 1 & 30 \\
\hline 369 & $\begin{array}{l}\text { Galb1-4GIcNAcb1-2Mana1-6(Galb1-4GIcNAcb1-4(Galb1-4GlcNAcb1-2)Mana1-3)Manb1-4GIcNAcb1-4GlcNAc- } \\
\text { Sp21 }\end{array}$ & 3 & 9 & 272 \\
\hline 487 & Neu5Aca2-6Galb1-4GIcNAcb1-6(Fuca1-2Galb1-4(Fuca1-3)GlcNAcb1-3)Galb1-4Glc-Sp21 & 3 & 3 & 96 \\
\hline 527 & Gala1-3Galb1-3GlcNAcb1-2Mana-Sp0 & 3 & 2 & 57 \\
\hline 313 & Mana1-6Manb-Sp10 & 3 & 0 & 4 \\
\hline 52 & GlcNAcb1-2Mana1-6(GlcNAcb1-2Mana1-3)Manb1-4GlcNAcb1-4GlcNAcb-Sp12 & 3 & 2 & 68 \\
\hline 598 & GlcNAcb1-3Galb1-4GlcNAcb1-3Galb1-4GlcNAcb1-3GalNAca-Sp14 & 3 & 7 & 203 \\
\hline 529 & Neu5Aca2-3Galb1-3GalNAcb1-4Galb1-4Glcb-Sp0 & 3 & 3 & 96 \\
\hline 42 & (6S)Galb1-4Glcb-Sp0 & 3 & 1 & 41 \\
\hline 287 & Neu5Gca-Sp8 & 3 & 4 & 114 \\
\hline 289 & Galb1-3GIcNAcb1-3Galb1-3GIcNAcb-Sp0 & 3 & 3 & 105 \\
\hline 327 & $\begin{array}{l}\text { Galb1-4(Fuca1-3)GlcNAcb1-2Mana1-6(Galb1-4(Fuca1-3)GlcNAcb1-2Mana1-3)Manb1-4GlcNAcb1-4GlcNAcb- } \\
\text { Sp20 }\end{array}$ & 3 & 4 & 132 \\
\hline 144 & Galb1-3GalNAcb1-4(Neu5Aca2-3)Galb1-4GIcb-Sp0 & 3 & 4 & 127 \\
\hline 250 & Neu5Aca2-3Galb1-3GIcNAcb-Sp8 & 3 & 5 & 158 \\
\hline 391 & Galb1-3GlcNAcb1-3GalNAca-Sp14 & 3 & 5 & 172 \\
\hline 495 & Gala1-3Galb1-4GIcNAcb1-6GalNAca-Sp14 & 3 & 1 & 40 \\
\hline 573 & Neu5Aca2-8Neu5Aca2-3Galb1-3GalNAcb1-4(Neu5Aca2-3)Galb1-4Glc-Sp21 & 3 & 5 & 171 \\
\hline 539 & $\begin{array}{l}\text { GlcNAcb1-3Galb1-4GIcNAcb1-2Mana1-6(GlcNAcb1-3Galb1-4GIcNAcb1-2Mana1-3)Manb1-4GIcNAcb1- } \\
\text { 4GIcNAcb-Sp12 }\end{array}$ & 3 & 2 & 52 \\
\hline
\end{tabular}




\begin{tabular}{|c|c|c|c|c|}
\hline 100 & Gala1-2Galb-Sp8 & 3 & 4 & 139 \\
\hline 479 & Neu5Aca2-3Galb1-4GIcNAcb1-6GalNAca-Sp14 & 3 & 3 & 99 \\
\hline 436 & $\begin{array}{l}\text { Galb1-4GIcNAcb1-2Mana1-6(GlcNAcb1-4)(Galb1-4GIcNAcb1-4(Galb1-4GIcNAcb1-2)Mana1-3)Manb1- } \\
\text { 4GlcNAcb1-4GIcNAc-Sp21 }\end{array}$ & 3 & 2 & 64 \\
\hline 515 & (3S)GalNAcb1-4GIcNAc-Sp8 & 3 & 2 & 81 \\
\hline 249 & Neu5Aca2-3Galb1-3GIcNAcb-Sp0 & 3 & 4 & 138 \\
\hline 134 & GlcNAcb1-6(Galb1-3)GalNAca-Sp14 & 3 & 1 & 22 \\
\hline 122 & Gala1-4Galb1-4Glcb-Sp0 & 3 & 3 & 116 \\
\hline 141 & Galb1-3GalNAca-Sp16 & 3 & 7 & 233 \\
\hline 477 & Neu5Aca2-6Galb1-4GIcNAcb1-6(Galb1-3GIcNAcb1-3)Galb1-4GIcb-Sp21 & 3 & 2 & 74 \\
\hline 266 & Neu5Aca2-6GalNAcb1-4GlcNAcb-Sp0 & 3 & 4 & 134 \\
\hline 247 & Neu5Aca2-3Galb1-3GIcNAcb1-3Galb1-4GIcNAcb-Sp0 & 3 & 3 & 103 \\
\hline 90 & GalNAca1-3(Fuca1-2)Galb-Sp18 & 3 & 2 & 66 \\
\hline 74 & Fuca1-2Galb1-4GIcNAcb1-3Galb1-4GIcNAcb1-3Galb1-4GIcNAcb-Sp0 & 3 & 3 & 111 \\
\hline 133 & GlcNAcb1-6(Galb1-3)GalNAca-Sp8 & 3 & 2 & 79 \\
\hline 444 & (6S)Galb1-3(6S)GlcNAc-Sp0 & 3 & 4 & 136 \\
\hline 49 & Neu5,9Ac2a2-6Galb1-4GIcNAcb-Sp8 & 3 & 4 & 159 \\
\hline 107 & Gala1-3(Fuca1-2)Galb-Sp8 & 3 & 3 & 128 \\
\hline 173 & GlcNAca1-3Galb1-4GIcNAcb-Sp8 & 3 & 2 & 69 \\
\hline 17 & GlcNAcb-Sp8 & 3 & 4 & 173 \\
\hline 150 & Galb1-3GlcNAcb-Sp8 & 3 & 4 & 169 \\
\hline 546 & $\begin{array}{l}\text { GlcNAcb1-3Galb1-4GlcNAcb1-3Galb1-4GlcNAcb1-2Mana1-6(GlcNAcb1-3Galb1-4GlcNAcb1-3Galb1-4GlcNAcb1- } \\
\text { 2Mana1-3)Manb1-4GlcNAcb1-4GlcNAcb-Sp25 }\end{array}$ & 2 & 6 & 241 \\
\hline 138 & Neu5Aca2-6(Galb1-3)GlcNAcb1-4Galb1-4Glcb-Sp10 & 2 & 1 & 22 \\
\hline 23 & $6 S(3 S)$ Galb1-4GIcNAcb-Sp0 & 2 & 3 & 120 \\
\hline 308 & Neu5Aca2-6Galb1-4GIcNAcb1-2Mana1-6(GIcNAcb1-2Mana1-3)Manb1-4GIcNAcb1-4GIcNAcb-Sp12 & 2 & 4 & 179 \\
\hline 77 & Fuca1-2Galb1-4Glcb-Sp0 & 2 & 5 & 199 \\
\hline 303 & GlcNAcb1-6(Galb1-4GIcNAcb1-3)Galb1-4GIcNAc-Sp0 & 2 & 13 & 530 \\
\hline 544 & $\begin{array}{l}\text { Fuca1-2Galb1-4GIcNAcb1-3Galb1-4GIcNAcb1-2Mana1-6(Fuca1-2Galb1-4GIcNAcb1-3Galb1-4GIcNAcb1- } \\
\text { 2Mana1-3)Manb1-4GIcNAcb1-4GIcNAcb-Sp24 }\end{array}$ & 2 & 4 & 165 \\
\hline 87 & GalNAca1-3(Fuca1-2)Galb1-4Glcb-Sp0 & 2 & 2 & 88 \\
\hline 19 & Galb1-4GlcNAcb1-6(Galb1-4GlcNAcb1-3)GalNAca-Sp8 & 2 & 5 & 197 \\
\hline 244 & Neu5Aca2-6(Neu5Aca2-3Galb1-3)GalNAca-Sp14 & 2 & 1 & 43 \\
\hline 405 & Gala1-3Galb1-4GlcNAcb1-3GalNAca-Sp14 & 2 & 5 & 209 \\
\hline 177 & GlcNAcb1-6(GlcNAcb1-3)GalNAca-Sp14 & 2 & 5 & 223 \\
\hline 113 & Gala1-3Galb1-4(Fuca1-3)GlcNAcb-Sp8 & 2 & 4 & 189 \\
\hline 531 & $\begin{array}{l}\text { Galb1-4GIcNAcb1-2 Mana1-6(GlcNAcb1-4)(Galb1-4GlcNAcb1-2Mana1-3)Manb1-4GlcNAcb1-4(Fuca1-6)GlcNAc- } \\
\text { Sp21 }\end{array}$ & 2 & 2 & 65 \\
\hline 230 & Neu5Aca2-3(6S)Galb1-4(Fuca1-3)GlcNAcb-Sp8 & 2 & 1 & 65 \\
\hline
\end{tabular}




\begin{tabular}{|c|c|c|c|c|}
\hline 242 & Neu5Aca2-3Galb1-3(6S)GalNAca-Sp8 & 2 & 4 & 161 \\
\hline 130 & Fuca1-4(Galb1-3)GIcNAcb-Sp8 & 2 & 5 & 226 \\
\hline 48 & $\mathrm{Neu} 5,9 \mathrm{Ac}_{2} \mathrm{a}-\mathrm{Sp} 8$ & 2 & 1 & 38 \\
\hline 178 & GlcNAcb1-6(GlcNAcb1-3)Galb1-4GlcNAcb-Sp8 & 2 & 4 & 171 \\
\hline 172 & Galb1-4GIcb-Sp8 & 2 & 8 & 379 \\
\hline 296 & 4S(3S)Galb1-4GlcNAcb-Sp0 & 2 & 5 & 230 \\
\hline 72 & Fuca1-2Galb1-4(Fuca1-3)GlcNAcb-Sp8 & 2 & 6 & 321 \\
\hline 509 & Galb1-3GlcNAca1-3Galb1-4GIcNAcb-Sp8 & 2 & 4 & 195 \\
\hline 294 & Neu5Aca2-3Galb1-3GIcNAcb1-3Galb1-3GIcNAcb-Sp0 & 2 & 2 & 104 \\
\hline 50 & Mana1-6(Mana1-3)Manb1-4GlcNAcb1-4GlcNAcb-Sp12 & 2 & 5 & 249 \\
\hline 163 & Galb1-4GIcNAcb1-3Galb1-4GIcNAcb-Sp0 & 2 & 4 & 195 \\
\hline 198 & Glcb1-4Glcb-Sp8 & 2 & 1 & 46 \\
\hline 314 & Mana1-6(Mana1-3)Mana1-6(Mana1-3)Manb-Sp10 & 2 & 2 & 105 \\
\hline 62 & Fuca1-2Galb1-3GalNAca-Sp14 & 2 & 2 & 106 \\
\hline 555 & Neu5Gca2-8Neu5Gca2-3Galb1-4GIcNAcb1-3Galb1-4GIcNAc-Sp0 & 2 & 2 & 137 \\
\hline 252 & Neu5Aca2-3Galb1-4(Fuca1-3)(6S)GlcNAcb-Sp8 & 2 & 5 & 300 \\
\hline 187 & GlcNAcb1-6(GlcNAcb1-4)GalNAca-Sp8 & 2 & 4 & 257 \\
\hline 46 & Neu5Aca2-3(6S)Galb1-4GIcNAcb-Sp8 & 2 & 3 & 215 \\
\hline 358 & KDNa2-3Galb1-4Glc-Sp0 & 2 & 8 & 524 \\
\hline 111 & Gala1-3GalNAca-Sp16 & 2 & 5 & 351 \\
\hline 519 & GalNAca1-3(Fuca1-2)Galb1-4GIcNAcb1-6GalNAc-Sp14 & 2 & 2 & 100 \\
\hline 430 & Fuca1-3GlcNAcb1-6(Galb1-4GlcNAcb1-3)Galb1-4Glc-Sp21 & 2 & 1 & 49 \\
\hline 298 & (6P)Glcb-Sp10 & 2 & 4 & 256 \\
\hline 374 & $\begin{array}{l}\text { Gala1-3(Fuca1-2)Galb1-3GlcNAcb1-2Mana1-6(Gala1-3(Fuca1-2)Galb1-3GlcNAcb1-2Mana1-3)Manb1- } \\
\text { 4GlcNAcb1-4GlcNAcb-Sp20 }\end{array}$ & 1 & 3 & 207 \\
\hline 422 & Fuca1-2Galb1-3GIcNAcb1-3GalNAc-Sp14 & 1 & 2 & 107 \\
\hline 275 & Neu5Aca2-8Neu5Aca-Sp8 & 1 & 2 & 107 \\
\hline 330 & Neu5Aca2-6Galb1-4GIcNAcb1-3Galb1-3GIcNAcb-Sp0 & 1 & 4 & 272 \\
\hline 243 & Neu5Aca2-6(Neu5Aca2-3Galb1-3)GalNAca-Sp8 & 1 & 5 & 386 \\
\hline 255 & Neu5Aca2-3Galb1-4(Fuca1-3)GlcNAcb-Sp8 & 1 & 3 & 201 \\
\hline 278 & Neu5Acb2-6GalNAca-Sp8 & 1 & 4 & 316 \\
\hline 306 & GalNAcb1-3Galb-Sp8 & 1 & 3 & 230 \\
\hline 162 & Galb1-4GIcNAcb1-3Galb1-4GIcNAcb1-3Galb1-4GIcNAcb-Sp0 & 1 & 4 & 295 \\
\hline 185 & GlcNAcb1-3Galb1-4Glcb-Sp0 & 1 & 2 & 182 \\
\hline 447 & Galb1-4(Fuca1-3)GIcNAcb1-6GalNAc-Sp14 & 1 & 5 & 432 \\
\hline 332 & Neu5Aca2-6Galb1-4GIcNAcb1-3Galb1-4GIcNAcb1-3Galb1-4GIcNAcb-Sp0 & 1 & 3 & 231 \\
\hline 59 & Fuca1-2Galb1-3GalNAcb1-3Gala1-4Galb1-4Glcb-Sp9 & 1 & 5 & 446 \\
\hline 312 & MurNAcb1-4GlcNAcb-Sp10 & 1 & 3 & 241 \\
\hline 79 & Fuca1-3GIcNAcb-Sp8 & 1 & 1 & 113 \\
\hline
\end{tabular}




\begin{tabular}{|c|c|c|c|c|}
\hline 101 & Gala1-3(Fuca1-2)Galb1-3GIcNAcb-Sp0 & 1 & 4 & 335 \\
\hline 219 & (3S)Galb1-4(Fuca1-3)(6S)GlcNAcb-Sp8 & 1 & 3 & 308 \\
\hline 258 & Neu5Aca2-3Galb1-4GIcNAcb1-3Galb1-4GIcNAcb1-3Galb1-4GIcNAcb-Sp0 & 1 & 8 & 743 \\
\hline 465 & $\begin{array}{l}\text { Neu5Aca2-6Galb1-4GIcNAcb1-6(Neu5Aca2-6Galb1-4GlcNAcb1-2)Mana1-6(GlcNAcb1-4)(Neu5Aca2-6Galb1- } \\
\text { 4GIcNAcb1-2Mana1-3)Manb1-4GIcNAcb1-4GIcNAcb-Sp21 }\end{array}$ & 1 & 0 & 44 \\
\hline 235 & Neu5Aca2-6(Neu5Aca2-3)GalNAca-Sp8 & 1 & 3 & 328 \\
\hline 20 & Galb1-4GIcNAcb1-6(Galb1-4GIcNAcb1-3)GalNAc-Sp14 & 1 & 2 & 232 \\
\hline 283 & Neu5Gca2-3Galb1-4GlcNAcb-Sp0 & 1 & 2 & 177 \\
\hline 181 & GlcNAcb1-3Galb-Sp8 & 1 & 5 & 526 \\
\hline 462 & $\begin{array}{l}\text { Neu5Aca2-3Galb1-4GlcNAcb1-6(Neu5Aca2-3Galb1-4GIcNAcb1-2)Mana1-6(GlcNAcb1-4)(Neu5Aca2-3Galb1- } \\
\text { 4GIcNAcb1-4(Neu5Aca2-3Galb1-4GIcNAcb1-2)Mana1-3)Manb1-4GlcNAcb1-4GIcNAcb-Sp21 }\end{array}$ & 1 & 4 & 451 \\
\hline 280 & Neu5Gca2-3Galb1-3(Fuca1-4)GIcNAcb-Sp0 & 1 & 7 & 785 \\
\hline 117 & Gala1-3Galb1-4GIc-Sp10 & 1 & 3 & 409 \\
\hline 16 & GlcNAcb-Sp0 & 1 & 3 & 420 \\
\hline 535 & GIcNAcb1-3Galb1-4GIcNAcb1-6(GlcNAcb1-3)Galb1-4GlcNAc-Sp0 & 1 & 5 & 574 \\
\hline 199 & Glcb1-6Glcb-Sp8 & 1 & 9 & 1245 \\
\hline 441 & Neu5Aca2-3Galb1-4GIcNAcb1-3Galb-Sp8 & 1 & 4 & 554 \\
\hline 212 & Mana1-2Mana1-6(Mana1-2Mana1-3)Mana1-6(Mana1-2Mana1-2Mana1-3)Manb1-4GIcNAcb1-4GIcNAcb-Sp12 & 1 & 13 & 2006 \\
\hline 128 & Galb1-3(Fuca1-4)GIcNAc-Sp0 & 0 & 5 & 1075 \\
\hline 126 & Galb1-3(Fuca1-4)GlcNAcb1-3Galb1-4(Fuca1-3)GlcNAcb-Sp0 & 0 & 3 & 744 \\
\hline 437 & $\begin{array}{l}\text { Galb1-4GIcNAcb1-6(Galb1-4GIcNAcb1-2)Mana1-6(GlcNAcb1-4)(Galb1-4GIcNAcb1-2Mana1-3)Manb1- } \\
\text { 4GIcNAcb1-4GIcNAc-Sp21 }\end{array}$ & 0 & 2 & 473 \\
\hline 89 & GalNAca1-3(Fuca1-2)Galb-Sp8 & 0 & 5 & 1394 \\
\hline 410 & Galb1-4GIcNAcb1-6(Neu5Aca2-6Galb1-3GlcNAcb1-3)Galb1-4Glc-Sp21 & 0 & 4 & 1181 \\
\hline 18 & $\mathrm{GlcN}(\mathrm{Gc}) \mathrm{b}-\mathrm{Sp} 8$ & 0 & 3 & 809 \\
\hline 207 & Mana1-2Mana1-2Mana1-3Mana-Sp9 & 0 & 4 & 2298 \\
\hline 557 & Neu5Aca2-8Neu5Aca2-3Galb1-4GIcNAc-Sp0 & 0 & 10 & 5068 \\
\hline 499 & Fuca1-2Galb1-4GIcNAcb1-2Mana-Sp0 & 0 & 3 & 1850 \\
\hline 523 & GalNAca1-3(Fuca1-2)Galb1-4 GlcNAcb1-2Mana-Sp0 & 0 & 3 & 1691 \\
\hline 354 & Galb1-3GIcNAcb1-2Mana1-6(Galb1-3GlcNAcb1-2Mana1-3)Manb1-4GIcNAcb1-4(Fuca1-6)GIcNAcb-Sp22 & 0 & 5 & 4550 \\
\hline 189 & GIcNAcb1-4GlcNAcb1-4GIcNAcb1-4GlcNAcb1-4GIcNAcb1-4GIcNAcb1-Sp8 & 0 & 4 & 3451 \\
\hline 286 & Neu5Gca2-6Galb1-4GIcNAcb-Sp0 & 0 & 2 & -7228 \\
\hline
\end{tabular}




\begin{tabular}{|c|c|c|c|c|}
\hline 56 & $\begin{array}{l}\text { Neu5Aca2-6Galb1-4GIcNAcb1-2Mana1-6(Neu5Aca2-6Galb1-4GIcNAcb1-2Man-a1-3)Manb1-4GIcNAcb1- } \\
\text { 4GIcNAcb-Sp21 }\end{array}$ & 0 & 2 & -4906 \\
\hline 604 & GlcNAcb1-6(Neu5Aca2-3Galb1-3)GalNAca-Sp14 & 0 & 2 & -1137 \\
\hline 209 & Mana1-2Mana1-3Mana-Sp9 & 0 & 1 & -270 \\
\hline 297 & (6S)Galb1-4(6S)GlcNAcb-Sp0 & 0 & 3 & -1198 \\
\hline 592 & Neu5Aca2-3Galb1-4GIcNAcb1-3Galb1-4GIcNAcb1-3GalNAca-Sp14 & 0 & 2 & -571 \\
\hline 526 & Neu5Aca2-3Galb1-3GIcNAcb1-2Mana-Sp0 & 0 & 4 & -883 \\
\hline 190 & GlcNAcb1-4GIcNAcb1-4GlcNAcb1-4GIcNAcb1-4GlcNAcb1-Sp8 & 0 & 2 & -416 \\
\hline 271 & Neu5Aca2-6Galb1-4GlcNAcb1-3Galb1-4GlcNAcb-Sp0 & -1 & 6 & -1175 \\
\hline 40 & (4S)Galb1-4GlcNAcb-Sp8 & -1 & 2 & -335 \\
\hline 567 & Galb1-3GIcNAcb1-6(Galb1-3)GalNAc-Sp14 & -1 & 2 & -338 \\
\hline 412 & Neu5Aca2-3Galb1-3GalNAcb1-4(Neu5Aca2-8Neu5Aca2-3)Galb1-4Glcb-Sp0 & -1 & 5 & -707 \\
\hline 542 & $\begin{array}{l}\text { Galb1-4GIcNAcb1-3Galb1-4GIcNAcb1-2Mana1-6(Galb1-4GIcNAcb1-3Galb1-4GIcNAcb1-2Mana1-3)Manb1- } \\
\text { 4GlcNAcb1-4GlcNAcb-Sp24 }\end{array}$ & -1 & 7 & -1003 \\
\hline 73 & Fuca1-2Galb1-4GIcNAcb1-3Galb1-4GIcNAcb-Sp0 & -1 & 4 & -519 \\
\hline 597 & Neu5Aca2-6Galb1-4GIcNAcb1-3Galb1-4GIcNAcb1-3GalNAca-Sp14 & -1 & 2 & -218 \\
\hline 285 & Neu5Gca2-6GalNAca-Sp0 & -1 & 4 & -539 \\
\hline 139 & Galb1-3GalNAca-Sp8 & -1 & 3 & -399 \\
\hline 413 & Gala1-3(Fuca1-2)Galb1-4GIcNAcb1-3GalNAca-Sp14 & -1 & 4 & -412 \\
\hline 276 & Neu5Aca2-8Neu5Aca2-3Galb1-4Glcb-Sp0 & -1 & 1 & -115 \\
\hline 316 & Mana1-2Mana1-6(Mana1-2Mana1-3)Mana1-6(Mana1-2Mana1-2Mana1-3)Mana-Sp9 & -1 & 6 & -550 \\
\hline 507 & $\begin{array}{l}\text { GlcNAcb1-6(GlcNAcb1-2)Mana1-6(GlcNAcb1-4)(GlcNAcb1-4(GlcNAcb1-2)Mana1-3)Manb1-4GlcNAcb1-4(Fuca1- } \\
\text { 6)GlcNAc-Sp21 }\end{array}$ & -1 & 3 & -276 \\
\hline 222 & Fuca1-2(6S)Galb1-4(6S)Glcb-Sp0 & -1 & 2 & -175 \\
\hline 254 & Neu5Aca2-3Galb1-4(Fuca1-3)GlcNAcb-Sp0 & -1 & 2 & -196 \\
\hline 14 & Manb-Sp8 & -1 & 5 & -380 \\
\hline 241 & Neu5Aca2-3Galb1-4(Neu5Aca2-3Galb1-3)GlcNAcb-Sp8 & -1 & 4 & -313 \\
\hline 408 & GalNAcb1-3Gala1-6Galb1-4Glcb-Sp8 & -2 & 3 & -194 \\
\hline 248 & Fuca1-2(6S)Galb1-4Glcb-Sp0 & -2 & 2 & -150 \\
\hline
\end{tabular}




\begin{tabular}{|c|c|c|c|c|}
\hline 389 & Fuca1-2Galb1-3GalNAca1-3(Fuca1-2)Galb1-4Glcb-Sp0 & -2 & 3 & -181 \\
\hline 305 & Galb1-4GIcNAcb1-6Galb1-4GIcNAcb-Sp0 & -2 & 2 & -126 \\
\hline 154 & Galb1-4(Fuca1-3)GIcNAcb1-3Galb1-4(Fuca1-3)GIcNAcb1-3Galb1-4(Fuca1-3)GIcNAcb-Sp0 & -2 & 3 & -161 \\
\hline 220 & Fuca1-2(6S)Galb1-4GIcNAcb-Sp0 & -2 & 4 & -170 \\
\hline 551 & $\begin{array}{l}\text { Galb1-3GlcNAcb1-3Galb1-4GIcNAcb1-2Mana1-6(Galb1-3GlcNAcb1-3Galb1-4GlcNAcb1-2Mana1-3)Manb1- } \\
\text { 4GlcNAcb1-4GlcNAc-Sp25 }\end{array}$ & -2 & 9 & -353 \\
\hline 284 & Neu5Gca2-3Galb1-4Glcb-Sp0 & -3 & 3 & -90 \\
\hline 464 & $\begin{array}{l}\text { Neu5Aca2-6Galb1-4GlcNAcb1-4Mana1-6(GlcNAcb1-4)(Neu5Aca2-6Galb1-4GlcNAcb1-4(Neu5Aca2-6Galb1- } \\
\text { 4GIcNAcb1-2)Mana1-3)Manb1-4GlcNAcb1-4GlcNAcb-Sp21 }\end{array}$ & -3 & 2 & -58 \\
\hline 553 & Neu5Aca2-8Neu5Gca2-3Galb1-4GIcNAc-Sp0 & -4 & 4 & -98 \\
\hline 506 & GalNAca1-3(Fuca1-2)Galb1-3GIcNAcb1-6GalNAca-Sp14 & -4 & 8 & -182 \\
\hline 599 & Galb1-4GlcNAcb1-3Galb1-3GalNAca-Sp14 & -4 & 5 & -115 \\
\hline 317 & Neu5Aca2-3Galb1-4GlcNAcb1-6(Neu5Aca2-3Galb1-3)GalNAca-Sp14 & -5 & 9 & -170 \\
\hline 528 & GalNAcb1-4GIcNAcb1-2Mana-Sp0 & -6 & 4 & -60 \\
\hline
\end{tabular}

Supporting data 6.rAAL-I144G_200ug_16389_v5.1_DATA

\begin{tabular}{|c|c|c|c|c|}
\hline $\begin{array}{l}\text { Chart } \\
\text { Number }\end{array}$ & rAAL-1144G 200ug/ml Slide\#16389 CFG\#2809 v5.1 Alexa488 5/27/12 HJ & Average RFU & StDev & $\% \mathrm{CV}$ \\
\hline 29 & (3S)Galb1-3GalNAca-Sp8 & 1442 & 99 & 7 \\
\hline 570 & (3S)GlcAb1-3Galb1-4GlcNAcb1-2Mana-Sp0 & 989 & 53 & 5 \\
\hline 569 & (3S)GIcAb1-3Galb1-4GIcNAcb1-3Galb1-4GIc-Sp0 & 798 & 88 & 11 \\
\hline 242 & Neu5Aca2-3Galb1-3(6S)GalNAca-Sp8 & 354 & 61 & 17 \\
\hline 363 & Gala1-3Galb1-4GIcNAcb1-2Mana1-6(Gala1-3Galb1-4GIcNAcb1-2Mana1-3)Manb1-4GIcNAcb1-4GIcNAcb-Sp20 & 325 & 16 & 5 \\
\hline 223 & Neu5Aca2-3Galb1-3GalNAca-Sp8 & 271 & 15 & 6 \\
\hline 560 & Gala1-3Galb1-4GIcNAcb1-2Mana1-6(Gala1-3Galb1-4GIcNAcb1-2Mana1-3)Manb1-4GIcNAcb1-4GIcNAc-Sp24 & 183 & 28 & 15 \\
\hline 573 & Neu5Aca2-8Neu5Aca2-3Galb1-3GalNAcb1-4(Neu5Aca2-3)Galb1-4Glc-Sp21 & 180 & 39 & 21 \\
\hline 604 & GIcNAcb1-6(Neu5Aca2-3Galb1-3)GalNAca-Sp14 & 159 & 70 & 44 \\
\hline 459 & $\begin{array}{l}\text { Neu5Aca2-3Galb1-4GIcNAcb1-2Mana1-6(GIcNAcb1-4)(Neu5Aca2-3Galb1-4GIcNAcb1-2Mana1-3)Manb1- } \\
\text { 4GlcNAcb1-4GlcNAcb-Sp21 }\end{array}$ & 151 & 25 & 17 \\
\hline 26 & (3S)Galb1-4(6S)Glcb-Sp0 & 141 & 56 & 40 \\
\hline 246 & Neu5Aca2-3Galb1-3GalNAcb1-3Gala1-4Galb1-4GIcb-Sp0 & 127 & 24 & 19 \\
\hline 234 & Neu5Aca2-3Galb1-3GalNAcb1-4(Neu5Aca2-3)Galb1-4Glcb-Sp0 & 125 & 15 & 12 \\
\hline 448 & Galb1-4GIcNAcb1-2Mana-Sp0 & 106 & 18 & 17 \\
\hline 543 & $\begin{array}{l}\text { Neu5Gca2-3Galb1-4GlcNAcb1-3Galb1-4GlcNAcb1-2Mana1-6(Neu5Gca2-3Galb1-4GIcNAcb1-3Galb1- } \\
\text { 4GIcNAcb1-2Mana1-3)Manb1-4GlcNAcb1-4GIcNAcb-Sp24 }\end{array}$ & 98 & 36 & 37 \\
\hline 317 & Neu5Aca2-3Galb1-4GlcNAcb1-6(Neu5Aca2-3Galb1-3)GalNAca-Sp14 & 64 & 18 & 29 \\
\hline 467 & Gala1-3(Fuca1-2)Galb1-3GalNAca-Sp8 & 44 & 17 & 38 \\
\hline 224 & Neu5Aca2-3Galb1-3GalNAca-Sp14 & 36 & 7 & 18 \\
\hline 460 & $\begin{array}{l}\text { Neu5Aca2-3Galb1-4GIcNAcb1-4Mana1-6(GlcNAcb1-4)(Neu5Aca2-3Galb1-4GlcNAcb1-4(Neu5Aca2-3Galb1- } \\
\text { 4GIcNAcb1-2)Mana1-3)Manb1-4GIcNAcb1-4GIcNAcb-Sp21 }\end{array}$ & 35 & 15 & 42 \\
\hline
\end{tabular}




\begin{tabular}{|c|c|c|c|c|}
\hline 34 & (3S)Galb1-4(6S)GIcNAcb-Sp0 & 34 & 9 & 26 \\
\hline 325 & $\begin{array}{l}\text { Neu5Aca2-3Galb1-4GlcNAcb1-2Mana1-6(Neu5Aca2-3Galb1-4GlcNAcb1-2Mana1-3)Manb1-4GIcNAcb1- } \\
\text { 4GIcNAcb-Sp12 }\end{array}$ & 33 & 13 & 37 \\
\hline 372 & $\begin{array}{l}\text { Gala1-3Galb1-4(Fuca1-3)GlcNAcb1-2Mana1-6(Gala1-3Galb1-4(Fuca1-3)GlcNAcb1-2Mana1-3)Manb1- } \\
\text { 4GIcNAcb1-4GlcNAcb-Sp20 }\end{array}$ & 26 & 11 & 43 \\
\hline 483 & $\begin{array}{l}\text { Neu5Aca2-3Galb1-4GIcNAcb1-2Mana1-6(Neu5Aca2-3Galb1-4GlcNAcb1-2Mana1-3)Manb1-4GIcNAcb1- } \\
\text { 4(Fuca1-6)GIcNAcb-Sp24 }\end{array}$ & 24 & 12 & 50 \\
\hline 362 & $\begin{array}{l}\text { Fuca1-2Galb1-4(Fuca1-3)GlcNAcb1-2Mana1-6(Fuca1-2Galb1-4(Fuca1-3)GlcNAcb1-2Mana1-3)Manb1- } \\
\text { 4GIcNAcb1-4GlcNAb-Sp20 }\end{array}$ & 23 & 12 & 51 \\
\hline 446 & $\begin{array}{l}\text { Fuca1-2Galb1-4(Fuca1-3)GIcNAcb1-2Mana1-6(Fuca1-2Galb1-4(Fuca1-3)GIcNAcb1-4(Fuca1-2Galb1-4(Fuca1- } \\
\text { 3)GIcNAcb1-2)Mana1-3)Manb1-4GIcNAcb1-4GIcNAcb-Sp12 }\end{array}$ & 22 & 3 & 15 \\
\hline 462 & $\begin{array}{l}\text { Neu5Aca2-3Galb1-4GIcNAcb1-6(Neu5Aca2-3Galb1-4GIcNAcb1-2)Mana1-6(GlcNAcb1-4)(Neu5Aca2-3Galb1- } \\
\text { 4GIcNAcb1-4(Neu5Aca2-3Galb1-4GIcNAcb1-2)Mana1-3)Manb1-4GIcNAcb1-4GIcNAcb-Sp21 }\end{array}$ & 22 & 13 & 58 \\
\hline 583 & $\begin{array}{l}\text { GlcNAcb1-3Galb1-4GIcNAcb1-3Galb1-4GIcNAcb1-6(GlcNAcb1-3Galb1-4GlcNAcb1-3Galb1-4GIcNAb1- } \\
\text { 2)Mana1-6(GIcNAcb1-3Galb1-4GIcNAcb1-3Galb1-4GIcNAcb1-2Mana1-3)Manb1-4GIcNAcb1-4(Fuca1- } \\
\text { 6)GIcNAcb-Sp24 }\end{array}$ & 22 & 8 & 39 \\
\hline 585 & $\begin{array}{l}\text { GlcNAcb1-3Galb1-4GlcNAcb1-3Galb1-4GlcNAcb1-3Galb1-4GlcNAcb1-6(GlcNAcb1-3Galb1-4GlcNAcb1- } \\
\text { 3Galb1-4GIcNAcb1-3Galb1-4GlcNAb1-2)Mana1-6(GlcNAcb1-3Galb1-4GlcNAcb1-3Galb1-4GlcNAcb1-3Galb1- } \\
\text { 4GlcNAcb1-2Mana1-3)Manb1-4GlcNAcb1-4(Fuca1-6)GlcNAcb-Sp24 }\end{array}$ & 22 & 14 & 66 \\
\hline 37 & (3S)Galb1-4GlcNAcb-Sp8 & 20 & 11 & 55 \\
\hline 232 & GalNAcb1-4(Neu5Aca2-3)Galb1-4GlcNAcb-Sp8 & 20 & 8 & 42 \\
\hline 481 & Neu5Aca2-6Galb1-4 GlcNAcb1-6(Neu5Aca2-6Galb1-4GIcNAcb1-3)GalNAca-Sp14 & 20 & 12 & 62 \\
\hline 72 & Fuca1-2Galb1-4(Fuca1-3)GlcNAcb-Sp8 & 19 & 13 & 67 \\
\hline 547 & $\begin{array}{l}\text { Galb1-4GIcNAcb1-3Galb1-4GIcNAcb1-3Galb1-4GlcNAcb1-2Mana1-6(Galb1-4GlcNAcb1-3Galb1-4GlcNAcb1- } \\
\text { 3Galb1-4GIcNAcb1-2Mana1-3)Manb1-4GlcNAcb1-4GIcNAcb-Sp12 }\end{array}$ & 19 & 20 & 105 \\
\hline 215 & Mana1-6(Mana1-3)Mana1-6(Mana1-2Mana1-3)Manb1-4GlcNAcb1-4GIcNAcb-Sp12 & 18 & 5 & 26 \\
\hline 555 & Neu5Gca2-8Neu5Gca2-3Galb1-4GIcNAcb1-3Galb1-4GIcNAc-Sp0 & 17 & 9 & 52 \\
\hline 122 & Gala1-4Galb1-4Glcb-Sp0 & 17 & 9 & 53 \\
\hline 610 & Galb1-3GalNAcb1-4(Neu5Aca2-8Neu5Aca2-8Neu5Aca2-3)Galb1-4Glcb-Sp21 & 17 & 6 & 35 \\
\hline 27 & (3S)Galb1-4(6S)Glcb-Sp8 & 17 & 10 & 62 \\
\hline 572 & $\begin{array}{l}\text { Galb1-3GlcNAcb1-3Galb1-4GlcNAcb1-6(Galb1-3GlcNAcb1-3Galb1-4GlcNAb1-2)Mana1-6(Galb1-3GlcNAcb1- } \\
\text { 3Galb1-4GIcNAcb1-2Mana1-3)Manb1-4GIcNAcb1-4(Fuca1-6)GlcNAcb-Sp24 }\end{array}$ & 16 & 8 & 50 \\
\hline 588 & $\begin{array}{l}\text { Galb1-4GlcNAcb1-3Galb1-4GlcNAcb1-3Galb1-4GlcNAcb1-3Galb1-4GlcNAcb1-3Galb1-4GIcNAcb1-6(Galb1- } \\
\text { 4GlcNAcb1-3Galb1-4GlcNAcb1-3Galb1-4GlcNAcb1-3Galb1-4GlcNAcb1-3Galb1-4GlcNAb1-2)Mana1-6(Galb1- } \\
\text { 4GlcNAcb1-3Galb1-4GlcNAcb1-3Galb1-4GlcNAcb1-3Galb1-4GlcNAcb1-3Galb1-4GlcNAcb1-2Mana1-3)Manb1- } \\
\text { 4GlcNAcb1-4(Fuca1-6)GlcNAcb-Sp24 }\end{array}$ & 16 & 3 & 18 \\
\hline 606 & $\begin{array}{l}\text { Neu5Aca2-6Galb1-4GlcNAcb1-3Galb1-4GIcNAcb1-3Galb1-4GlcNAcb1-2Mana1-6(Neu5Aca2-6Galb1- } \\
\text { 4GIcNAcb1-3Galb1-4GIcNAcb1-3Galb1-4GIcNAcb1-2Mana1-3)Manb1-4GIcNAcb1-4GIcNAcb-Sp12 }\end{array}$ & 16 & 4 & 22 \\
\hline 524 & Galb1-3GIcNAcb1-2Mana-Sp0 & 16 & 14 & 87 \\
\hline 370 & $\begin{array}{l}\text { GalNAca1-3(Fuca1-2)Galb1-4GIcNAcb1-2Mana1-6(GalNAca1-3(Fuca1-2)Galb1-4GIcNAcb1-2Mana1-3)Manb1- } \\
\text { 4GIcNAcb1-4GIcNAcb-Sp20 }\end{array}$ & 16 & 7 & 44 \\
\hline 267 & Neu5Aca2-6Galb1-4(6S)GlcNAcb-Sp8 & 16 & 15 & 95 \\
\hline 207 & Mana1-2Mana1-2Mana1-3Mana-Sp9 & 16 & 15 & 98 \\
\hline
\end{tabular}




\begin{tabular}{|c|c|c|c|c|}
\hline 283 & Neu5Gca2-3Galb1-4GlcNAcb-Sp0 & 16 & 11 & 71 \\
\hline 345 & Neu5Aca2-6Galb1-4GIcNAcb1-2Mana1-6(Mana1-3)Manb1-4GIcNAcb1-4GIcNAc-Sp12 & 16 & 4 & 26 \\
\hline 24 & (3S)Galb1-4(Fuca1-3)(6S)Glc-Sp0 & 15 & 6 & 42 \\
\hline 578 & $\begin{array}{l}\text { GlcNAcb1-3Galb1-4GlcNAcb1-3Galb1-4GlcNAcb1-3Galb1-4GlcNAcb1-2Mana1-6(GlcNAcb1-3Galb1- } \\
\text { 4GlcNAcb1-3Galb1-4GlcNAcb1-3Galb1-4GlcNAcb1-2Mana1-3)Manb1-4GlcNAcb1-4(Fuca1-6)GlcNAcb-Sp24 }\end{array}$ & 15 & 6 & 40 \\
\hline 211 & Mana1-2Mana1-6(Mana1-3)Mana1-6(Mana1-2Mana1-2Mana1-3)Manb1-4GIcNAcb1-4GIcNAcb-Sp12 & 15 & 13 & 85 \\
\hline 355 & (6S)GIcNAcb1-3Galb1-4GIcNAcb-Sp0 & 15 & 7 & 49 \\
\hline 360 & Fuca1-2Galb1-3GlcNAcb1-2Mana1-6(Fuca1-2Galb1-3GlcNAcb1-2Mana1-3)Manb1-4GlcNAcb1-4GlcNAcb-Sp20 & 15 & 12 & 80 \\
\hline 324 & Galb1-3GIcNAcb1-2Mana1-6(Galb1-3GIcNAcb1-2Mana1-3)Manb1-4GIcNAcb1-4GIcNAcb-Sp19 & 15 & 10 & 67 \\
\hline 403 & Gala1-4Galb1-3GIcNAcb1-2Mana1-6(Gala1-4Galb1-3GlcNAcb1-2Mana1-3)Manb1-4GIcNAcb1-4GIcNAcb-Sp19 & 15 & 6 & 43 \\
\hline 33 & (3S)Galb1-4(Fuca1-3)GlcNAc-Sp8 & 15 & 12 & 84 \\
\hline 182 & GlcNAcb1-3Galb1-4GlcNAcb-Sp0 & 14 & 14 & 99 \\
\hline 205 & KDNa2-3Galb1-3GIcNAcb-Sp0 & 14 & 1 & 8 \\
\hline 559 & $\begin{array}{l}\text { Galb1-4GlcNAcb1-3Galb1-4GIcNAcb1-6(Galb1-4GlcNAcb1-3Galb1-4GlcNAcb1-2)Mana1-6(Galb1-4GlcNAcb1- } \\
\text { 3Galb1-4GlcNAcb1-2Mana1-3)Mana1-4GlcNAcb1-4GlcNAc-Sp24 }\end{array}$ & 14 & 6 & 39 \\
\hline 591 & Galb1-4GIcNAcb1-3Galb1-4GIcNAcb1-6(Galb1-4GIcNAcb1-3Galb1-4GIcNAcb1-3)GalNAca-Sp14 & 14 & 10 & 74 \\
\hline 179 & GlcNAcb1-3GalNAca-Sp8 & 14 & 10 & 69 \\
\hline 576 & $\begin{array}{l}\text { GlcNAcb1-3Galb1-4GlcNAcb1-3Galb1-4GIcNAcb1-2Mana1-6(GIcNAcb1-3Galb1-4GIcNAcb1-3Galb1- } \\
\text { 4GIcNAcb1-2Mana1-3)Manb1-4GIcNAcb1-4(Fuca1-6)GIcNAcb-Sp24 }\end{array}$ & 14 & 11 & 79 \\
\hline 188 & GlcNAcb1-4Galb1-4GlcNAcb-Sp8 & 14 & 8 & 57 \\
\hline 424 & GalNAca1-3(Fuca1-2)Galb1-3GlcNAcb1-3GalNAc-Sp14 & 14 & 11 & 80 \\
\hline 213 & Mana1-6(Mana1-3)Mana-Sp9 & 14 & 6 & 44 \\
\hline 227 & Neu5Aca2-8Neu5Aca2-8Neu5Aca2-3Galb1-4Glcb-Sp0 & 14 & 3 & 25 \\
\hline 333 & Gala1-4Galb1-4GlcNAcb1-3Galb1-4Glcb-Sp0 & 14 & 4 & 32 \\
\hline 542 & $\begin{array}{l}\text { Galb1-4GIcNAcb1-3Galb1-4GIcNAcb1-2Mana1-6(Galb1-4GIcNAcb1-3Galb1-4GIcNAcb1-2Mana1-3)Manb1- } \\
\text { 4GlcNAcb1-4GIcNAcb-Sp24 }\end{array}$ & 13 & 13 & 95 \\
\hline 609 & GIcNAcb1-3Fuca-Sp21 & 13 & 7 & 49 \\
\hline 298 & (6P)Glcb-Sp10 & 13 & 8 & 58 \\
\hline 43 & (6S)Galb1-4Glcb-Sp8 & 13 & 8 & 59 \\
\hline 185 & GlcNAcb1-3Galb1-4Glcb-Sp0 & 13 & 3 & 23 \\
\hline 470 & Glca1-4Glca1-4Glca1-4GIcb-Sp10 & 13 & 14 & 110 \\
\hline 253 & Neu5Aca2-3Galb1-4(Fuca1-3)GlcNAcb1-3Galb1-4(Fuca1-3)GlcNAcb1-3Galb1-4(Fuca1-3)GlcNAcb-Sp0 & 13 & 10 & 75 \\
\hline 512 & (6S)GalNAcb1-4GlcNAc-Sp8 & 13 & 16 & 122 \\
\hline 587 & $\begin{array}{l}\text { GlcNAcb1-3Galb1-4GIcNAcb1-3Galb1-4GlcNAcb1-3Galb1-4GlcNAcb1-3Galb1-4GlcNAcb1-6(GlcNAcb1- } \\
\text { 3Galb1-4GlcNAcb1-3Galb1-4GIcNAcb1-3Galb1-4GIcNAcb1-3Galb1-4GIcNAb1-2)Mana1-6(GIcNAcb1-3Galb1- } \\
\text { 4GIcNAcb1-3Galb1-4GIcNAcb1-3Galb1-4GlcNAcb1-3Galb1-4GIcNAcb1-2Mana1-3)Manb1-4GIcNAcb1- } \\
\text { 4(Fuca1-6)GIcNAcb-Sp24 }\end{array}$ & 13 & 4 & 34 \\
\hline 475 & GlcNAcb1-6(GlcNAcb1-2)Mana1-6(GlcNAcb1-2Mana1-3)Manb1-4GIcNAcb1-4(Fuca1-6)GlcNAcb-Sp24 & 13 & 9 & 74 \\
\hline 25 & (3S)Galb1-4Glcb-Sp8 & 13 & 6 & 45 \\
\hline 119 & Gala1-4(Fuca1-2)Galb1-4GIcNAcb-Sp8 & 13 & 15 & 120 \\
\hline 513 & (3S)GalNAcb1-4(3S)GIcNAc-Sp8 & 13 & 8 & 67 \\
\hline 5 & GalNAca-Sp15 & 13 & 3 & 21 \\
\hline 407 & Galb1-3GIcNAca1-6Galb1-4GIcNAcb-Sp0 & 13 & 6 & 49 \\
\hline
\end{tabular}




\begin{tabular}{|c|c|c|c|c|}
\hline 432 & GlcNAcb1-2Mana1-6(GlcNAcb1-4)(GIcNAcb1-4(GlcNAcb1-2)Mana1-3)Manb1-4GlcNAcb1-4GlcNAc-Sp21 & 13 & 6 & 48 \\
\hline 318 & $\begin{array}{l}\text { Neu5Aca2-6Galb1-4GIcNAcb1-2Mana1-6(Neu5Aca2-3Galb1-4GIcNAcb1-2Mana1-3)Manb1-4GIcNAcb1- } \\
\text { 4GIcNAcb-Sp12 }\end{array}$ & 13 & 5 & 39 \\
\hline 490 & Galb1-3(Fuca1-4)GlcNAcb1-6GalNAca-Sp14 & 13 & 5 & 38 \\
\hline 248 & Fuca1-2(6S)Galb1-4Glcb-Sp0 & 12 & 8 & 64 \\
\hline 69 & Fuca1-2Galb1-4(Fuca1-3)GIcNAcb1-3Galb1-4(Fuca1-3)GIcNAcb-Sp0 & 12 & 3 & 28 \\
\hline 568 & $\begin{array}{l}\text { Galb1-4GIcNAcb1-3Galb1-4GlcNAcb1-3Galb1-4GlcNAcb1-3Galb1-4GlcNAcb1-3Galb1-4GlcNAcb1-3Galb1- } \\
\text { 4GIcNAcb1-2Mana1-6(Galb1-4GlcNAcb1-3Galb1-4GlcNAcb1-3Galb1-4GIcNAcb1-3Galb1-4GlcNAcb1-3Galb1- } \\
\text { 4GlcNAcb1-3Galb1-4GlcNAcb1-2Mana1-3)Manb1-4GlcNAcb1-4GlcNAcb-Sp25 }\end{array}$ & 12 & 7 & 58 \\
\hline 418 & GalNAca1-3(Fuca1-2)Galb1-4(Fuca1-3)GlcNAcb1-3GalNAc-Sp14 & 12 & 7 & 53 \\
\hline 35 & (3S)Galb1-4(6S)GlcNAcb-Sp8 & 12 & 3 & 27 \\
\hline 96 & GalNAcb1-3Gala1-4Galb1-4GIcNAcb-Sp0 & 12 & 3 & 23 \\
\hline 359 & KDNa2-3Galb1-3GalNAca-Sp14 & 12 & 5 & 43 \\
\hline 12 & Galb-Sp8 & 12 & 12 & 101 \\
\hline 443 & (6S)Galb1-3GIcNAcb-Sp0 & 12 & 6 & 53 \\
\hline 299 & Neu5Aca2-3Galb1-4(Fuca1-3)GIcNAcb1-6(Galb1-3)GalNAca-Sp14 & 12 & 13 & 108 \\
\hline 545 & $\begin{array}{l}\text { GlcNAcb1-3Galb1-4GIcNAcb1-3Galb1-4GIcNAcb1-2Mana1-6(GlcNAcb1-3Galb1-4GIcNAcb1-3Galb1- } \\
\text { 4GIcNAcb1-2Mana1-3)Manb1-4GIcNAcb1-4GIcNAcb-Sp12 }\end{array}$ & 12 & 8 & 66 \\
\hline 541 & $\begin{array}{l}\text { Galb1-4GIcNAcb1-3Galb1-4GIcNAcb1-2Mana1-6(Galb1-4GIcNAcb1-3Galb1-4GIcNAcb1-2Mana1-3)Manb1- } \\
\text { 4GIcNAcb1-4GlcNAcb-Sp12 }\end{array}$ & 12 & 8 & 72 \\
\hline 368 & Galb1-4(Fuca1-3)GlcNAcb1-6(Fuca1-2Galb1-4GIcNAcb1-3)Galb1-4GIc-Sp21 & 12 & 6 & 52 \\
\hline 228 & GalNAcb1-4(Neu5Aca2-8Neu5Aca2-3)Galb1-4Glcb-Sp0 & 12 & 4 & 35 \\
\hline 381 & Galb1-3GalNAca1-3(Fuca1-2)Galb1-4GIcNAc-Sp0 & 12 & 11 & 96 \\
\hline 36 & (3S)Galb1-4GIcNAcb-Sp0 & 12 & 6 & 55 \\
\hline 482 & $\begin{array}{l}\text { Neu5Aca2-6Galb1-4GIcNAcb1-2Mana1-6(Neu5Aca2-6Galb1-4GIcNAcb1-2Mana1-3)Manb1-4GIcNAcb1- } \\
\text { 4(Fuca1-6)GlcNAcb-Sp24 }\end{array}$ & 12 & 3 & 29 \\
\hline 584 & $\begin{array}{l}\text { Galb1-4GlcNAcb1-3Galb1-4GIcNAcb1-3Galb1-4GlcNAcb1-6(Galb1-4GIcNAcb1-3Galb1-4GIcNAcb1-3Galb1- } \\
\text { 4GIcNAb1-2)Mana1-6(Galb1-4GlcNAcb1-3Galb1-4GlcNAcb1-3Galb1-4GlcNAcb1-2Mana1-3)Manb1- } \\
\text { 4GIcNAcb1-4(Fuca1-6)GlcNAcb-Sp24 }\end{array}$ & 11 & 6 & 50 \\
\hline 219 & (3S)Galb1-4(Fuca1-3)(6S)GlcNAcb-Sp8 & 11 & 11 & 92 \\
\hline 84 & (3S)Galb1-4(Fuca1-3)Glcb-Sp0 & 11 & 5 & 44 \\
\hline 511 & (6S)(4S)GalNAcb1-4GlcNAc-Sp8 & 11 & 5 & 43 \\
\hline 235 & Neu5Aca2-6(Neu5Aca2-3)GalNAca-Sp8 & 11 & 9 & 79 \\
\hline 582 & $\begin{array}{l}\text { Galb1-4GIcNAcb1-3Galb1-4GlcNAcb1-6(Galb1-4GIcNAcb1-3Galb1-4GlcNAb1-2)Mana1-6(Galb1-4GIcNAcb1- } \\
\text { 3Galb1-4GIcNAcb1-2Mana1-3)Manb1-4GlcNAcb1-4(Fuca1-6)GlcNAcb-Sp24 }\end{array}$ & 11 & 4 & 34 \\
\hline 261 & Neu5Aca2-3Galb1-4GlcNAcb1-3Galb1-4GlcNAcb-Sp0 & 11 & 10 & 91 \\
\hline 589 & Galb1-4GIcNAcb1-3Galb1-4GIcNAcb1-3GalNAca-Sp14 & 11 & 3 & 31 \\
\hline 430 & Fuca1-3GIcNAcb1-6(Galb1-4GIcNAcb1-3)Galb1-4Glc-Sp21 & 11 & 10 & 86 \\
\hline 485 & $\begin{array}{l}\text { Galb1-4GlcNAcb1-6(Galb1-4GlcNAcb1-2)Mana1-6(Galb1-4GlcNAcb1-2Mana1-3)Manb1-4GIcNAcb1-4(Fuca1- } \\
\text { 6)GlcNAcb-Sp24 }\end{array}$ & 11 & 11 & 103 \\
\hline 52 & GlcNAcb1-2Mana1-6(GlcNAcb1-2Mana1-3)Manb1-4GIcNAcb1-4GlcNAcb-Sp12 & 11 & 3 & 30 \\
\hline 321 & Neu5Aca2-8Neu5Acb-Sp17 & 11 & 1 & 8 \\
\hline 468 & Gala1-3(Fuca1-2)Galb1-3GalNAcb-Sp8 & 11 & 4 & 40 \\
\hline
\end{tabular}




\begin{tabular}{|c|c|c|c|c|}
\hline 118 & Gala1-3Galb-Sp8 & 11 & 5 & 44 \\
\hline 441 & Neu5Aca2-3Galb1-4GIcNAcb1-3Galb-Sp8 & 11 & 9 & 85 \\
\hline 143 & Galb1-3GalNAcb1-3Gala1-4Galb1-4Glcb-Sp0 & 11 & 7 & 65 \\
\hline 383 & Galb1-4(Fuca1-3)GIcNAcb1-6(Galb1-3GlcNAcb1-3)Galb1-4Glc-Sp21 & 11 & 7 & 67 \\
\hline 121 & Gala1-4Galb1-4GlcNAcb-Sp8 & 11 & 5 & 49 \\
\hline 428 & $\begin{array}{l}\text { Galb1-3GIcNAcb1-6(Galb1-3GIcNAcb1-2)Mana1-6(Galb1-3GIcNAcb1-2Mana1-3)Manb1-4GlcNAcb1-4GlcNAcb- } \\
\text { Sp19 }\end{array}$ & 11 & 10 & 95 \\
\hline 241 & Neu5Aca2-3Galb1-4(Neu5Aca2-3Galb1-3)GIcNAcb-Sp8 & 11 & 6 & 61 \\
\hline 343 & GIcNAca1-4Galb1-4GIcNAcb1-3Galb1-4GIcNAcb-Sp0 & 11 & 5 & 50 \\
\hline 494 & Fuca1-2Galb1-4GlcNAcb1-6GalNAca-Sp14 & 11 & 8 & 75 \\
\hline 351 & Mana1-6(Galb1-4GIcNAcb1-2Mana1-3)Manb1-4GIcNAcb1-4GlcNAcb-Sp12 & 11 & 6 & 56 \\
\hline 349 & Galb1-4GIcNAcb1-2Mana1-3Manb1-4GIcNAcb1-4GIcNAc-Sp12 & 11 & 7 & 66 \\
\hline 250 & Neu5Aca2-3Galb1-3GlcNAcb-Sp8 & 11 & 8 & 71 \\
\hline 151 & Galb1-4(Fuca1-3)GlcNAcb-Sp0 & 11 & 8 & 78 \\
\hline 352 & GlcNAcb1-2Mana1-6(GlcNAcb1-2Mana1-3)Manb1-4GIcNAcb1-4(Fuca1-6)GlcNAcb-Sp22 & 11 & 6 & 54 \\
\hline 474 & $\begin{array}{l}\text { Neu5Aca2-3Galb1-3GIcNAcb1-6(Neu5Aca2-3Galb1-3GIcNAcb1-2)Mana1-6(Neu5Aca2-3Galb1-3GIcNAcb1- } \\
\text { 2Mana1-3)Manb1-4GIcNAcb1-4GIcNAcb-Sp19 }\end{array}$ & 11 & 11 & 100 \\
\hline 579 & $\begin{array}{l}\text { Galb1-4GlcNAcb1-3Galb1-4GIcNAcb1-3Galb1-4GIcNAcb1-3Galb1-4GlcNAcb1-2Mana1-6(Galb1-4GIcNAcb1- } \\
\text { 3Galb1-4GIcNAcb1-3Galb1-4GIcNAcb1-3Galb1-4GIcNAcb1-2Mana1-3)Manb1-4GlcNAcb1-4(Fuca1-6)GlcNAcb- } \\
\text { Sp24 }\end{array}$ & 10 & 3 & 29 \\
\hline 361 & Fuca1-2Galb1-4GIcNAcb1-2Mana1-6(Fuca1-2Galb1-4GIcNAcb1-2Mana1-3)Manb1-4GIcNAcb1-4GIcNAcb-Sp20 & 10 & 7 & 66 \\
\hline 364 & Galb1-4GIcNAcb1-2Mana1-6(Mana1-3)Manb1-4GIcNAcb1-4GlcNAcb-Sp12 & 10 & 7 & 65 \\
\hline 173 & GIcNAca1-3Galb1-4GIcNAcb-Sp8 & 10 & 5 & 48 \\
\hline 244 & Neu5Aca2-6(Neu5Aca2-3Galb1-3)GalNAca-Sp14 & 10 & 9 & 82 \\
\hline 521 & Gala1-3Galb1-4GlcNAcb1-2Mana-Sp0 & 10 & 8 & 79 \\
\hline 590 & Galb1-4GIcNAcb1-3Galb1-4GIcNAcb1-6(Galb1-3)GalNAca-Sp14 & 10 & 6 & 59 \\
\hline 508 & $\begin{array}{l}\text { Galb1-4GIcNAcb1-6(Galb1-4GIcNAcb1-2)Mana1-6(GlcNAcb1-4)Galb1-4GIcNAcb1-4(Gal b1-4GIcNAcb1- } \\
\text { 2)Mana1-3)Manb1-4GIcNAcb1-4(Fuca1-6)GIcNAc-Sp21 }\end{array}$ & 10 & 9 & 84 \\
\hline 169 & Galb1-4GIcNAcb-Sp8 & 10 & 2 & 22 \\
\hline 73 & Fuca1-2Galb1-4GIcNAcb1-3Galb1-4GIcNAcb-Sp0 & 10 & 6 & 61 \\
\hline 130 & Fuca1-4(Galb1-3)GlcNAcb-Sp8 & 10 & 3 & 30 \\
\hline 220 & Fuca1-2(6S)Galb1-4GIcNAcb-Sp0 & 10 & 8 & 80 \\
\hline 391 & Galb1-3GlcNAcb1-3GalNAca-Sp14 & 10 & 3 & 30 \\
\hline 102 & Gala1-3(Fuca1-2)Galb1-3GlcNAcb-Sp8 & 10 & 10 & 98 \\
\hline 178 & GIcNAcb1-6(GIcNAcb1-3)Galb1-4GIcNAcb-Sp8 & 10 & 7 & 68 \\
\hline 95 & GalNAcb1-3(Fuca1-2)Galb-Sp8 & 10 & 8 & 75 \\
\hline 195 & Glca1-4Glcb-Sp8 & 10 & 2 & 25 \\
\hline 540 & $\begin{array}{l}\text { GIcNAcb1-3Galb1-4GIcNAcb1-2Mana1-6(GlcNAcb1-3Galb1-4GIcNAcb1-2Mana1-3)Manb1-4GIcNAcb1- } \\
\text { 4GIcNAcb-Sp25 }\end{array}$ & 10 & 5 & 50 \\
\hline 478 & Neu5Aca2-3Galb1-4GIcNAcb1-2Mana-Sp0 & 10 & 10 & 105 \\
\hline 306 & GalNAcb1-3Galb-Sp8 & 10 & 4 & 42 \\
\hline 258 & Neu5Aca2-3Galb1-4GIcNAcb1-3Galb1-4GIcNAcb1-3Galb1-4GIcNAcb-Sp0 & 10 & 8 & 79 \\
\hline 280 & Neu5Gca2-3Galb1-3(Fuca1-4)GlcNAcb-Sp0 & 10 & 10 & 100 \\
\hline
\end{tabular}




\begin{tabular}{|c|c|c|c|c|}
\hline 546 & $\begin{array}{l}\text { GlcNAcb1-3Galb1-4GlcNAcb1-3Galb1-4GIcNAcb1-2Mana1-6(GIcNAcb1-3Galb1-4GlcNAcb1-3Galb1- } \\
\text { 4GIcNAcb1-2Mana1-3)Manb1-4GlcNAcb1-4GIcNAcb-Sp25 }\end{array}$ & 10 & 8 & 86 \\
\hline 63 & Fuca1-2Galb1-3GalNAcb1-4(Neu5Aca2-3)Galb1-4Glcb-Sp0 & 10 & 6 & 62 \\
\hline 461 & $\begin{array}{l}\text { Neu5Aca2-3Galb1-4GlcNAcb1-6(Neu5Aca2-3Galb1-4GlcNAcb1-2)Mana1-6(GlcNAcb1-4)(Neu5Aca2-3Galb1- } \\
\text { 4GIcNAcb1-2Mana1-3)Manb1-4GIcNAcb1-4GlcNAcb-Sp21 }\end{array}$ & 10 & 7 & 71 \\
\hline 145 & Galb1-3GalNAcb1-4Galb1-4Glcb-Sp8 & 10 & 4 & 41 \\
\hline 365 & $\begin{array}{l}\text { Fuca1-4(Galb1-3)GlcNAcb1-2Mana1-6(Fuca1-4(Galb1-3)GlcNAcb1-2Mana1-3)Manb1-4GlcNAcb1-4(Fuca1- } \\
\text { 6)GlcNAcb-Sp22 }\end{array}$ & 10 & 8 & 81 \\
\hline 350 & Galb1-4GlcNAcb1-2Mana1-6Manb1-4GIcNAcb1-4GIcNAc-Sp12 & 10 & 3 & 26 \\
\hline 315 & Mana1-2Mana1-6(Mana1-3)Mana1-6(Mana1-2Mana1-2Mana1-3)Mana-Sp9 & 10 & 9 & 97 \\
\hline 419 & $\begin{array}{l}\text { Galb1-4(Fuca1-3)GlcNAcb1-2Mana1-6(Galb1-4(Fuca1-3)GlcNAcb1-2Mana1-3)Manb1-4GlcNAcb1-4(Fuca1- } \\
\text { 6)GlcNAcb-Sp22 }\end{array}$ & 10 & 11 & 116 \\
\hline 54 & Galb1-4GIcNAcb1-2Mana1-6(Galb1-4GIcNAcb1-2Mana1-3)Manb1-4GIcNAcb1-4GIcNAcb-Sp12 & 9 & 4 & 45 \\
\hline 257 & Neu5Aca2-3Galb1-4(Fuca1-3)GlcNAcb1-3Galb1-4GIcNAcb-Sp8 & 9 & 7 & 74 \\
\hline 439 & Galb1-4Galb-Sp10 & 9 & 4 & 42 \\
\hline 487 & Neu5Aca2-6Galb1-4GIcNAcb1-6(Fuca1-2Galb1-4(Fuca1-3)GlcNAcb1-3)Galb1-4Glc-Sp21 & 9 & 10 & 108 \\
\hline 209 & Mana1-2Mana1-3Mana-Sp9 & 9 & 9 & 97 \\
\hline 558 & $\begin{array}{l}\text { GlcNAcb1-3Galb1-4GlcNAcb1-6(GlcNAcb1-3Galb1-4GlcNAcb1-2)Mana1-6(GlcNAcb1-3Galb1-4GlcNAcb1- } \\
\text { 2Man a1-3)Manb1-4GIcNAcb1-4GlcNAc-Sp24 }\end{array}$ & 9 & 9 & 91 \\
\hline 183 & GlcNAcb1-3Galb1-4GlcNAcb-Sp8 & 9 & 5 & 52 \\
\hline 323 & Neu5Gcb2-6Galb1-4GlcNAc-Sp8 & 9 & 5 & 53 \\
\hline 586 & $\begin{array}{l}\text { Galb1-4GlcNAcb1-3Galb1-4GIcNAcb1-3Galb1-4GlcNAcb1-3Galb1-4GlcNAcb1-6(Galb1-4GlcNAcb1-3Galb1- } \\
\text { 4GIcNAcb1-3Galb1-4GlcNAcb1-3Galb1-4GIcNAb1-2)Mana1-6(Galb1-4GlcNAcb1-3Galb1-4GlcNAcb1-3Galb1- } \\
\text { 4GlcNAcb1-3Galb1-4GlcNAcb1-2Mana1-3)Manb1-4GlcNAcb1-4(Fuca1-6)GlcNAcb-Sp24 }\end{array}$ & 9 & 4 & 40 \\
\hline 21 & GlcNAcb1-6(GlcNAcb1-4)(GlcNAcb1-3)GIcNAc-Sp8 & 9 & 12 & 126 \\
\hline 409 & Gala1-3(Fuca1-2)Galb1-4(Fuca1-3)Glcb-Sp21 & 9 & 5 & 53 \\
\hline 449 & Fuca1-2Galb1-4GlcNAcb1-6(Fuca1-2Galb1-4GlcNAcb1-3)GalNAc-Sp14 & 9 & 4 & 41 \\
\hline 353 & Galb1-4GIcNAcb1-2Mana1-6(Galb1-4GIcNAcb1-2Mana1-3)Manb1-4GlcNAcb1-4(Fuca1-6)GlcNAcb-Sp22 & 9 & 6 & 69 \\
\hline 68 & Fuca1-2Galb1-3GIcNAcb-Sp8 & 9 & 8 & 90 \\
\hline 9 & Neu5Aca-Sp8 & 9 & 7 & 75 \\
\hline 400 & Fuca1-2Galb1-4GIcNAcb1-3GalNAca-Sp14 & 9 & 1 & 13 \\
\hline 116 & Gala1-3Galb1-4GIcb-Sp0 & 9 & 7 & 80 \\
\hline 106 & Gala1-3(Fuca1-2)Galb1-4GIcb-Sp0 & 9 & 4 & 41 \\
\hline 607 & $\begin{array}{l}\text { Neu5Aca2-3Galb1-4GIcNAcb1-3Galb1-4GIcNAcb1-3Galb1-4GIcNAcb1-2Mana1-6(Neu5Aca2-3Galb1- } \\
\text { 4GIcNAcb1-3Galb1-4GIcNAcb1-3Galb1-4GIcNAcb1-2Mana1-3)Manb1-4GIcNAcb1-4GlcNAcb-Sp12 }\end{array}$ & 9 & 4 & 40 \\
\hline 484 & Mana1-6(Mana1-3)Manb1-4GIcNAcb1-4(Fuca1-6)GIcNAcb-Sp19 & 9 & 6 & 67 \\
\hline 18 & $\mathrm{GlcN}(\mathrm{Gc}) \mathrm{b}-\mathrm{Sp} 8$ & 9 & 5 & 59 \\
\hline 406 & Galb1-3GlcNAcb1-6Galb1-4GIcNAcb-Sp0 & 9 & 8 & 86 \\
\hline 565 & $\begin{array}{l}\text { Galb1-4GlcNAcb1-3Galb1-4GIcNAcb1-3Galb1-4GlcNAcb1-3Galb1-4GIcNAcb1-3Galb1-4GIcNAcb1-2Mana1- } \\
\text { 6(Galb1-4GIcNAcb1-3Galb1-4GIcNAcb1-3Galb1-4GlcNAcb1-3Galb1-4GIcNAcb1-3Galb1-4GIcNAcb1-2Mana1- } \\
\text { 3)Manb1-4GlcNAcb1-4GlcNAcb-Sp25 }\end{array}$ & 9 & 6 & 71 \\
\hline 124 & Gala1-6Glcb-Sp8 & 9 & 3 & 33 \\
\hline 201 & GlcAa-Sp8 & 9 & 10 & 109 \\
\hline
\end{tabular}




\begin{tabular}{|c|c|c|c|c|}
\hline 387 & $\begin{array}{l}\text { Galb1-4GIcNAcb1-6(Galb1-4GIcNAcb1-2)Mana1-6(Galb1-4GIcNAcb1-4(Galb1-4GlcNAcb1-2)Mana1-3)Manb1- } \\
\text { 4GIcNAcb1-4GIcNAcb-Sp21 }\end{array}$ & 9 & 6 & 73 \\
\hline 57 & $\begin{array}{l}\text { Neu5Aca2-6Galb1-4GIcNAcb1-2Mana1-6(Neu5Aca2-6Galb1-4GIcNAcb1-2Mana1-3)Manb1-4GIcNAcb1- } \\
\text { 4GIcNAcb-Sp24 }\end{array}$ & 9 & 9 & 105 \\
\hline 341 & GlcNAca1-4Galb1-4GlcNAcb1-3Galb1-4Glcb-Sp0 & 9 & 5 & 58 \\
\hline 574 & $\begin{array}{l}\text { GlcNAcb1-3Galb1-4GIcNAcb1-2Mana1-6(GlcNAcb1-3Galb1-4GIcNAcb1-2Mana1-3)Manb1-4GIcNAcb1- } \\
\text { 4(Fuca1-6)GIcNAcb-Sp24 }\end{array}$ & 9 & 7 & 79 \\
\hline 471 & Neu5Aca2-3Galb1-4GIcNAcb1-6(Neu5Aca2-3Galb1-4GIcNAcb1-3)GalNAca-Sp14 & 9 & 5 & 62 \\
\hline 557 & Neu5Aca2-8Neu5Aca2-3Galb1-4GIcNAc-Sp0 & 9 & 6 & 65 \\
\hline 577 & $\begin{array}{l}\text { Galb1-4GlcNAcb1-3Galb1-4GlcNAcb1-3Galb1-4GIcNAcb1-2Mana1-6(Galb1-4GlcNAcb1-3Galb1-4GlcNAcb1- } \\
\text { 3Galb1-4GlcNAcb1-2Mana1-3)Manb1-4GlcNAcb1-4(Fuca1-6)GlcNAcb-Sp24 }\end{array}$ & 9 & 3 & 40 \\
\hline 268 & Neu5Aca2-6Galb1-4GlcNAcb-Sp0 & 9 & 7 & 83 \\
\hline 532 & $\begin{array}{l}\text { Galb1-4GIcNAcb1-2 Mana1-6(Galb1-4GIcNAcb1-4)(Galb1-4GIcNAcb1-2Mana1-3)Manb1-4GIcNAcb1-4(Fuca1- } \\
\text { 6)GIcNAc-Sp21 }\end{array}$ & 9 & 15 & 175 \\
\hline 358 & KDNa2-3Galb1-4GIc-Sp0 & 9 & 4 & 49 \\
\hline 50 & Mana1-6(Mana1-3)Manb1-4GlcNAcb1-4GlcNAcb-Sp12 & 9 & 9 & 109 \\
\hline 473 & $\begin{array}{l}\text { Fuca1-2Galb1-3(Fuca1-4)GlcNAcb1-2Mana1-6(Fuca1-2Galb1-3(Fuca1-4)GlcNAcb1-2Mana1-3)Manb1- } \\
\text { 4GlcNAcb1-4(Fuca1-6)GlcNAcb1-4(Fuca1-6)GlcNAcb-Sp19 }\end{array}$ & 9 & 4 & 44 \\
\hline 39 & $(6 S)(4 S) G a l b 1-4 G I c N A c b-S p 0$ & 9 & 6 & 75 \\
\hline 285 & Neu5Gca2-6GalNAca-Sp0 & 9 & 9 & 106 \\
\hline 575 & $\begin{array}{l}\text { Galb1-4GIcNAcb1-3Galb1-4GIcNAcb1-2Mana1-6(Galb1-4GIcNAcb1-3Galb1-4GIcNAcb1-2Mana1-3)Manb1- } \\
\text { 4GIcNAcb1-4(Fuca1-6)GIcNAcb-Sp24 }\end{array}$ & 9 & 4 & 46 \\
\hline 501 & Fuca1-2(6S)Galb1-3(6S)GIcNAcb-Sp0 & 8 & 2 & 26 \\
\hline 436 & $\begin{array}{l}\text { Galb1-4GIcNAcb1-2Mana1-6(GIcNAcb1-4)(Galb1-4GIcNAcb1-4(Galb1-4GlcNAcb1-2)Mana1-3)Manb1- } \\
\text { 4GIcNAcb1-4GlcNAc-Sp21 }\end{array}$ & 8 & 8 & 95 \\
\hline 354 & Galb1-3GIcNAcb1-2Mana1-6(Galb1-3GIcNAcb1-2Mana1-3)Manb1-4GIcNAcb1-4(Fuca1-6)GIcNAcb-Sp22 & 8 & 6 & 74 \\
\hline 330 & Neu5Aca2-6Galb1-4GIcNAcb1-3Galb1-3GIcNAcb-Sp0 & 8 & 5 & 57 \\
\hline 498 & Gala1-3(Fuca1-2)Galb1-4GIcNAcb1-6GalNAca-Sp14 & 8 & 7 & 84 \\
\hline 88 & GlcNAcb1-3Galb1-3GalNAca-Sp8 & 8 & 2 & 30 \\
\hline 38 & (3S)Galb-Sp8 & 8 & 6 & 76 \\
\hline 226 & GalNAcb1-4(Neu5Aca2-8Neu5Aca2-8Neu5Aca2-3)Galb1-4Glcb-Sp0 & 8 & 6 & 72 \\
\hline 217 & Manb1-4GIcNAcb-Sp0 & 8 & 3 & 37 \\
\hline 369 & $\begin{array}{l}\text { Galb1-4GIcNAcb1-2Mana1-6(Galb1-4GIcNAcb1-4(Galb1-4GIcNAcb1-2)Mana1-3)Manb1-4GIcNAcb1-4GIcNAc- } \\
\text { Sp21 }\end{array}$ & 8 & 6 & 72 \\
\hline 110 & Gala1-3GalNAca-Sp8 & 8 & 4 & 51 \\
\hline 263 & Neu5Aca2-3Galb1-4Glcb-Sp0 & 8 & 7 & 84 \\
\hline 75 & Fuca1-2Galb1-4GIcNAcb-Sp0 & 8 & 12 & 144 \\
\hline 373 & $\begin{array}{l}\text { GalNAca1-3(Fuca1-2)Galb1-3GIcNAcb1-2Mana1-6(GalNAca1-3(Fuca1-2)Galb1-3GIcNAcb1-2Mana1-3)Manb1- } \\
\text { 4GIcNAcb1-4GIcNAcb-Sp20 }\end{array}$ & 8 & 6 & 71 \\
\hline 593 & GlcNAcb1-3Galb1-4GIcNAcb1-3GalNAca-Sp14 & 8 & 3 & 41 \\
\hline 596 & $\begin{array}{l}\text { Neu5Aca2-3Galb1-4GIcNAcb1-3Galb1-4GIcNAcb1-6(Neu5Aca2-3Galb1-4GIcNAcb1-3Galb1-4GIcNAcb1- } \\
\text { 3)GalNAca-Sp14 }\end{array}$ & 8 & 7 & 88 \\
\hline 236 & Neu5Aca2-3GalNAca-Sp8 & 8 & 9 & 108 \\
\hline
\end{tabular}




\begin{tabular}{|c|c|c|c|c|}
\hline 404 & Gala1-4Galb1-4GIcNAcb1-2Mana1-6(Gala1-4Galb1-4GIcNAcb1-2Mana1-3)Manb1-4GlcNAcb1-4GlcNAcb-Sp24 & 8 & 3 & 33 \\
\hline 374 & $\begin{array}{l}\text { Gala1-3(Fuca1-2)Galb1-3GlcNAcb1-2Mana1-6(Gala1-3(Fuca1-2)Galb1-3GlcNAcb1-2Mana1-3)Manb1- } \\
\text { 4GlcNAcb1-4GIcNAcb-Sp20 }\end{array}$ & 8 & 5 & 68 \\
\hline 146 & Galb1-3Galb-Sp8 & 8 & 6 & 79 \\
\hline 86 & GalNAca1-3(Fuca1-2)Galb1-4GIcNAcb-Sp8 & 8 & 8 & 105 \\
\hline 531 & $\begin{array}{l}\text { Galb1-4GIcNAcb1-2 Mana1-6(GlcNAcb1-4)(Galb1-4GlcNAcb1-2Mana1-3)Manb1-4GlcNAcb1-4(Fuca1- } \\
\text { 6)GlcNAc-Sp21 }\end{array}$ & 8 & 8 & 102 \\
\hline 270 & Neu5Aca2-6Galb1-4GIcNAcb1-3Galb1-4(Fuca1-3)GlcNAcb1-3Galb1-4(Fuca1-3)GlcNAcb-Sp0 & 8 & 8 & 100 \\
\hline 332 & Neu5Aca2-6Galb1-4GIcNAcb1-3Galb1-4GIcNAcb1-3Galb1-4GIcNAcb-Sp0 & 8 & 8 & 97 \\
\hline 162 & Galb1-4GlcNAcb1-3Galb1-4GlcNAcb1-3Galb1-4GlcNAcb-Sp0 & 8 & 6 & 77 \\
\hline 421 & GlcNAcb1-2(GlcNAcb1-6)Mana1-6(GlcNAcb1-2Mana1-3)Manb1-4GlcNAcb1-4GlcNAcb-Sp19 & 8 & 5 & 64 \\
\hline 605 & $\begin{array}{l}\text { Neu5Aca2-6Galb1-4GIcNAcb1-3Galb1-4GIcNAcb1-6(Neu5Aca2-6Galb1-4GlcNAcb1-3Galb1-4GlcNAcb1- } \\
\text { 3)GalNAca-Sp14 }\end{array}$ & 8 & 4 & 55 \\
\hline 378 & Neu5Aca2-3Galb1-4(Fuca1-3)GIcNAcb1-3GalNAca-Sp14 & 8 & 7 & 88 \\
\hline 602 & Neu5Aca2-6Galb1-4GIcNAcb1-6(Galb1-3)GalNAca-Sp14 & 8 & 9 & 120 \\
\hline 420 & $\begin{array}{l}\text { Fuca1-2Galb1-4GIcNAcb1-2Mana1-6(Fuca1-2Galb1-4GlcNAcb1-2Mana1-3)Manb1-4GlcNAcb1-4(Fuca1- } \\
\text { 6)GlcNAcb-Sp22 }\end{array}$ & 8 & 8 & 108 \\
\hline 15 & GalNAcb-Sp8 & 8 & 6 & 76 \\
\hline 80 & Fuca1-4GIcNAcb-Sp8 & 8 & 1 & 7 \\
\hline 243 & Neu5Aca2-6(Neu5Aca2-3Galb1-3)GalNAca-Sp8 & 8 & 6 & 81 \\
\hline 401 & Galb1-4(Fuca1-3)GIcNAcb1-3GalNAca-Sp14 & 8 & 6 & 75 \\
\hline 564 & $\begin{array}{l}\text { GlcNAcb1-3Galb1-4GlcNAcb1-3Galb1-4GIcNAcb1-3Galb1-4GlcNAcb1-3Galb1-4GlcNAcb1-2Mana1- } \\
\text { 6(GlcNAcb1-3Galb1-4GlcNAcb1-3Galb1-4GIcNAcb1-3Galb1-4GlcNAcb1-3Galb1-4GlcNAcb1-2Mana1-3)Manb1- } \\
\text { 4GlcNAcb1-4GlcNAcb-Sp25 }\end{array}$ & 8 & 10 & 132 \\
\hline 571 & $\begin{array}{l}\text { Galb1-3GlcNAcb1-3Galb1-4GIcNAcb1-3Galb1-4GIcNAcb1-6(Galb1-3GlcNAcb1-3Galb1-4GlcNAcb1-3Galb1- } \\
\text { 4GlcNAb1-2)Mana1-6(Galb1-3GIcNAcb1-3Galb1-4GlcNAcb1-3Galb1-4GIcNAcb1-2Mana1-3)Manb1- } \\
\text { 4GlcNAcb1-4(Fuca1-6)GlcNAcb-Sp24 }\end{array}$ & 8 & 2 & 32 \\
\hline 200 & G-ol-Sp8 & 8 & 3 & 36 \\
\hline 259 & Neu5Aca2-3Galb1-4GIcNAcb-Sp0 & 7 & 3 & 44 \\
\hline 357 & KDNa2-6Galb1-4GIcNAc-Sp0 & 7 & 6 & 82 \\
\hline 316 & Mana1-2Mana1-6(Mana1-2Mana1-3)Mana1-6(Mana1-2Mana1-2Mana1-3)Mana-Sp9 & 7 & 4 & 51 \\
\hline 108 & Gala1-3(Fuca1-2)Galb-Sp18 & 7 & 6 & 81 \\
\hline 516 & (4S)GalNAcb-Sp10 & 7 & 4 & 56 \\
\hline 514 & GalNAcb1-4(6S)GIcNAc-Sp8 & 7 & 9 & 118 \\
\hline 410 & Galb1-4GIcNAcb1-6(Neu5Aca2-6Galb1-3GIcNAcb1-3)Galb1-4Glc-Sp21 & 7 & 12 & 160 \\
\hline 94 & GalNAcb1-3GalNAca-Sp8 & 7 & 4 & 56 \\
\hline 62 & Fuca1-2Galb1-3GalNAca-Sp14 & 7 & 11 & 147 \\
\hline 336 & GalNAca1-3(Fuca1-2)Galb1-4GlcNAcb1-3Galb1-4GlcNAcb1-3Galb1-4GlcNAcb-Sp0 & 7 & 6 & 85 \\
\hline 595 & GlcNAcb1-3Galb1-4GlcNAcb1-6(GlcNAcb1-3Galb1-4GlcNAcb1-3)GalNAca-Sp14 & 7 & 5 & 64 \\
\hline 492 & (3S)Galb1-3(Fuca1-4)GIcNAcb-Sp0 & 7 & 3 & 47 \\
\hline 214 & Mana1-2Mana1-2Mana1-6(Mana1-3)Mana-Sp9 & 7 & 8 & 106 \\
\hline 507 & $\begin{array}{l}\text { GlcNAcb1-6(GlcNAcb1-2)Mana1-6(GlcNAcb1-4)(GlcNAcb1-4(GlcNAcb1-2)Mana1-3)Manb1-4GIcNAcb1- } \\
\text { 4(Fuca1-6)GlcNAc-Sp21 }\end{array}$ & 7 & 2 & 25 \\
\hline
\end{tabular}




\begin{tabular}{|c|c|c|c|c|}
\hline 51 & Mana1-6(Mana1-3)Manb1-4GIcNAcb1-4GIcNAcb-Sp13 & 7 & 4 & 61 \\
\hline 197 & Glca1-6Glca1-6GIcb-Sp8 & 7 & 4 & 53 \\
\hline 203 & GlcAb1-3Galb-Sp8 & 7 & 6 & 80 \\
\hline 78 & Fuca1-2Galb-Sp8 & 7 & 3 & 48 \\
\hline 561 & GlcNAcb1-3Galb1-4GIcNAcb1-6(GlcNAcb1-3Galb1-3)GalNAca-Sp14 & 7 & 2 & 25 \\
\hline 107 & Gala1-3(Fuca1-2)Galb-Sp8 & 7 & 6 & 80 \\
\hline 91 & GalNAca1-3GalNAcb-Sp8 & 7 & 8 & 116 \\
\hline 6 & Fuca-Sp8 & 7 & 4 & 53 \\
\hline 10 & Neu5Aca-Sp11 & 7 & 6 & 90 \\
\hline 2 & Glca-Sp8 & 7 & 2 & 36 \\
\hline 477 & Neu5Aca2-6Galb1-4GIcNAcb1-6(Galb1-3GIcNAcb1-3)Galb1-4Glcb-Sp21 & 7 & 2 & 36 \\
\hline 597 & Neu5Aca2-6Galb1-4GIcNAcb1-3Galb1-4GIcNAcb1-3GalNAca-Sp14 & 7 & 7 & 99 \\
\hline 356 & KDNa2-3Galb1-4(Fuca1-3)GIcNAc-Sp0 & 7 & 8 & 111 \\
\hline 123 & Gala1-4GlcNAcb-Sp8 & 7 & 6 & 88 \\
\hline 502 & Neu5Aca2-6GalNAcb1-4(6S)GIcNAcb-Sp8 & 7 & 9 & 131 \\
\hline 534 & Neu5Aca2-3Galb1-4(Fuca1-3)GIcNAcb1-2Mana-Sp0 & 7 & 4 & 65 \\
\hline 408 & GalNAcb1-3Gala1-6Galb1-4Glcb-Sp8 & 7 & 10 & 152 \\
\hline 194 & GIcNAcb1-6Galb1-4GIcNAcb-Sp8 & 7 & 2 & 31 \\
\hline 328 & Neu5,9Ac2a2-3Galb1-4GIcNAcb-Sp0 & 7 & 5 & 75 \\
\hline 371 & $\begin{array}{l}\text { Gala1-3(Fuca1-2)Galb1-4GlcNAcb1-2Mana1-6(Gala1-3(Fuca1-2)Galb1-4GIcNAcb1-2Mana1-3)Manb1- } \\
\text { 4GIcNAcb1-4GIcNAcb-Sp20 }\end{array}$ & 7 & 3 & 50 \\
\hline 326 & $\begin{array}{l}\text { Neu5Aca2-3Galb1-4GIcNAcb1-2Mana1-6(Neu5Aca2-6Galb1-4GIcNAcb1-2Mana1-3)Manb1-4GIcNAcb1- } \\
\text { 4GIcNAcb-Sp12 }\end{array}$ & 7 & 7 & 106 \\
\hline 98 & GalNAcb1-4GlcNAcb-Sp0 & 7 & 6 & 87 \\
\hline 60 & Fuca1-2Galb1-3(Fuca1-4)GIcNAcb-Sp8 & 7 & 3 & 39 \\
\hline 1 & Gala-Sp8 & 7 & 5 & 78 \\
\hline 254 & Neu5Aca2-3Galb1-4(Fuca1-3)GlcNAcb-Sp0 & 7 & 4 & 55 \\
\hline 331 & Neu5Aca2-3Galb1-3(Fuca1-4)GlcNAcb1-3Galb1-3(Fuca1-4)GlcNAcb-Sp0 & 7 & 2 & 35 \\
\hline 142 & Galb1-3GalNAcb-Sp8 & 7 & 7 & 107 \\
\hline 221 & Fuca1-2Galb1-4(6S)GIcNAcb-Sp8 & 7 & 10 & 149 \\
\hline 237 & Neu5Aca2-3GalNAcb1-4GlcNAcb-Sp0 & 7 & 4 & 61 \\
\hline 509 & Galb1-3GIcNAca1-3Galb1-4GIcNAcb-Sp8 & 6 & 6 & 90 \\
\hline 240 & Neu5Aca2-3Galb1-3(Fuca1-4)GlcNAcb1-3Galb1-4(Fuca1-3)GlcNAcb-Sp0 & 6 & 8 & 128 \\
\hline 59 & Fuca1-2Galb1-3GalNAcb1-3Gala1-4Galb1-4GIcb-Sp9 & 6 & 8 & 127 \\
\hline 210 & Mana1-6(Mana1-2Mana1-3)Mana1-6(Mana1-2Mana1-3)Manb1-4GIcNAcb1-4GIcNAcb-Sp12 & 6 & 10 & 164 \\
\hline 594 & GlcNAcb1-3Galb1-4GlcNAcb1-6(Galb1-3)GalNAca-Sp14 & 6 & 7 & 111 \\
\hline 489 & Gala1-3Galb1-3GIcNAcb1-6GalNAca-Sp14 & 6 & 6 & 99 \\
\hline 105 & Gala1-3(Fuca1-2)Galb1-4GIcNAc-Sp0 & 6 & 7 & 113 \\
\hline 13 & Glcb-Sp8 & 6 & 3 & 43 \\
\hline 346 & Mana1-6(Neu5Aca2-6Galb1-4GIcNAcb1-2Mana1-3)Manb1-4GIcNAcb1-4GIcNAc-Sp12 & 6 & 10 & 154 \\
\hline 553 & Neu5Aca2-8Neu5Gca2-3Galb1-4GIcNAc-Sp0 & 6 & 6 & 94 \\
\hline 140 & Galb1-3GalNAca-Sp14 & 6 & 4 & 70 \\
\hline 500 & Fuca1-2Galb1-3(6S)GIcNAcb-Sp0 & 6 & 5 & 74 \\
\hline
\end{tabular}




\begin{tabular}{|c|c|c|c|c|}
\hline 386 & Galb1-3GlcNAcb1-3Galb1-4(Fuca1-3)GlcNAcb1-6(Galb1-3GlcNAcb1-3)Galb1-4Glc-Sp21 & 6 & 3 & 44 \\
\hline 109 & Gala1-4(Gala1-3)Galb1-4GlcNAcb-Sp8 & 6 & 9 & 151 \\
\hline 491 & Neu5Aca2-3Galb1-3GIcNAcb1-6GalNAca-Sp14 & 6 & 8 & 136 \\
\hline 249 & Neu5Aca2-3Galb1-3GIcNAcb-Sp0 & 6 & 8 & 138 \\
\hline 55 & $\begin{array}{l}\text { Neu5Aca2-6Galb1-4GlcNAcb1-2Mana1-6(Neu5Aca2-6Galb1-4GlcNAcb1-2Mana1-3)Manb1-4GIcNAcb1- } \\
\text { 4GIcNAcb-Sp12 }\end{array}$ & 6 & 3 & 53 \\
\hline 114 & Gala1-3Galb1-3GlcNAcb-Sp0 & 6 & 1 & 24 \\
\hline 566 & GIcNAb1-3Galb1-3GalNAc-Sp14 & 6 & 11 & 179 \\
\hline 64 & Fuca1-2Galb1-3GalNAcb1-4(Neu5Aca2-3)Galb1-4Glcb-Sp9 & 6 & 2 & 31 \\
\hline 376 & Neu5Aca2-3Galb1-4GIcNAcb1-3GalNAc-Sp14 & 6 & 5 & 84 \\
\hline 307 & GlcAb1-3GlcNAcb-Sp8 & 6 & 6 & 104 \\
\hline 539 & $\begin{array}{l}\text { GlcNAcb1-3Galb1-4GlcNAcb1-2Mana1-6(GlcNAcb1-3Galb1-4GlcNAcb1-2Mana1-3)Manb1-4GlcNAcb1- } \\
\text { 4GlcNAcb-Sp12 }\end{array}$ & 6 & 6 & 104 \\
\hline 472 & $\begin{array}{l}\text { Fuca1-2Galb1-4(Fuca1-3)GIcNAcb1-2Mana1-6(Fuca1-2Galb1-4(Fuca1-3)GIcNAcb1-2Mana1-3)Manb1- } \\
\text { 4GIcNAcb1-4(Fuca1-6)GlcNAcb-Sp24 }\end{array}$ & 6 & 4 & 69 \\
\hline 519 & GalNAca1-3(Fuca1-2)Galb1-4GlcNAcb1-6GalNAc-Sp14 & 6 & 6 & 108 \\
\hline 417 & Gala1-3(Fuca1-2)Galb1-4(Fuca1-3)GlcNAcb1-3GalNAc-Sp14 & 6 & 8 & 136 \\
\hline 444 & (6S)Galb1-3(6S)GIcNAc-Sp0 & 6 & 4 & 72 \\
\hline 342 & GIcNAca1-4Galb1-4GIcNAcb1-3Galb1-4(Fuca1-3)GIcNAcb1-3Galb1-4(Fuca1-3)GIcNAcb-Sp0 & 6 & 4 & 65 \\
\hline 397 & GlcNAcb1-2Mana1-6(Galb1-4GlcNAcb1-2Mana1-3)Manb1-4GIcNAcb1-4GIcNAc-Sp12 & 6 & 9 & 152 \\
\hline 47 & (6S)GlcNAcb-Sp8 & 6 & 1 & 15 \\
\hline 347 & Neu5Aca2-6Galb1-4GIcNAcb1-2Mana1-6Manb1-4GIcNAcb1-4GIcNAc-Sp12 & 6 & 1 & 24 \\
\hline 518 & (6P)Galb1-4GlcNAcb-SP0 & 6 & 4 & 66 \\
\hline 152 & Galb1-4(Fuca1-3)GlcNAcb-Sp8 & 6 & 6 & 96 \\
\hline 454 & $\begin{array}{l}\text { GalNAca1-3(Fuca1-2)Galb1-4GIcNAcb1-2Mana1-6(GalNAca1-3(Fuca1-2)Galb1-4GlcNAcb1-2Mana1-3)Manb1- } \\
\text { 4GlcNAcb1-4(Fuca1-6)GlcNAcb-Sp22 }\end{array}$ & 6 & 2 & 43 \\
\hline 308 & Neu5Aca2-6Galb1-4GIcNAcb1-2Mana1-6(GIcNAcb1-2Mana1-3)Manb1-4GlcNAcb1-4GlcNAcb-Sp12 & 6 & 4 & 66 \\
\hline 295 & Neu5Aca2-3Galb1-4GlcNAcb1-3Galb1-3GlcNAcb-Sp0 & 6 & 2 & 29 \\
\hline 311 & GlcNAcb1-4GlcNAcb-Sp12 & 6 & 12 & 201 \\
\hline 167 & Galb1-4GIcNAcb1-6(Galb1-3)GalNAc-Sp14 & 6 & 7 & 115 \\
\hline 216 & Mana1-6(Mana1-3)Mana1-6(Mana1-3)Manb1-4GIcNAcb1-4GIcNAcb-Sp12 & 6 & 5 & 84 \\
\hline 450 & Gala1-3(Fuca1-2)Galb1-4GIcNAcb1-6(Gala1-3(Fuca1-2)Galb1-4GIcNAcb1-3)GalNAc-Sp14 & 6 & 4 & 64 \\
\hline 447 & Galb1-4(Fuca1-3)GlcNAcb1-6GalNAc-Sp14 & 6 & 7 & 122 \\
\hline 337 & Neu5Aca2-3Galb1-4(Fuca1-3)GlcNAcb1-6(Neu5Aca2-3Galb1-3)GalNAc-Sp14 & 6 & 5 & 92 \\
\hline 412 & Neu5Aca2-3Galb1-3GalNAcb1-4(Neu5Aca2-8Neu5Aca2-3)Galb1-4Glcb-Sp0 & 6 & 6 & 108 \\
\hline 141 & Galb1-3GalNAca-Sp16 & 6 & 8 & 135 \\
\hline 320 & GlcNAcb1-2Mana1-6(Neu5Aca2-6Galb1-4GIcNAcb1-2Mana1-3)Manb1-4GlcNAcb1-4GlcNAcb-Sp12 & 6 & 4 & 65 \\
\hline 476 & Galb1-3GIcNAcb1-2Mana1-6(GIcNAcb1-4)(Galb1-3GIcNAcb1-2Mana1-3)Manb1-4GIcNAcb1-4GIcNAcb-Sp21 & 6 & 5 & 88 \\
\hline 309 & GIcNAcb1-3Man-Sp10 & 6 & 6 & 108 \\
\hline 552 & Neu5Gca2-8Neu5Gca2-3Galb1-4GIcNAc-Sp0 & 6 & 4 & 68 \\
\hline 366 & Neu5Aca2-6GlcNAcb1-4GlcNAc-Sp21 & 6 & 6 & 111 \\
\hline 529 & Neu5Aca2-3Galb1-3GalNAcb1-4Galb1-4GIcb-Sp0 & 6 & 6 & 111 \\
\hline 275 & Neu5Aca2-8Neu5Aca-Sp8 & 6 & 9 & 158 \\
\hline
\end{tabular}




\begin{tabular}{|c|c|c|c|c|}
\hline 495 & Gala1-3Galb1-4GIcNAcb1-6GalNAca-Sp14 & 5 & 4 & 75 \\
\hline 76 & Fuca1-2Galb1-4GIcNAcb-Sp8 & 5 & 2 & 32 \\
\hline 339 & GlcNAca1-4Galb1-4GlcNAcb-Sp0 & 5 & 5 & 98 \\
\hline 28 & (3S)Galb1-3(Fuca1-4)GlcNAcb-Sp8 & 5 & 7 & 122 \\
\hline 31 & (3S)Galb1-3GlcNAcb-Sp8 & 5 & 4 & 65 \\
\hline 520 & Neu5Aca2-6Galb1-4GIcNAcb1-2Man-Sp0 & 5 & 2 & 43 \\
\hline 416 & Fuca1-2Galb1-4(Fuca1-3)GIcNAcb1-3GalNAca-Sp14 & 5 & 2 & 42 \\
\hline 290 & Galb1-4(Fuca1-3)(6S)GlcNAcb-Sp0 & 5 & 6 & 102 \\
\hline 380 & Galb1-3GalNAca1-3(Fuca1-2)Galb1-4GIc-Sp0 & 5 & 8 & 139 \\
\hline 388 & GlcNAcb1-2Mana1-6(GlcNAcb1-4(GlcNAcb1-2)Mana1-3)Manb1-4GIcNAcb1-4GIcNAc-Sp21 & 5 & 4 & 73 \\
\hline 385 & Galb1-4(Fuca1-3)GlcNAcb1-6(Fuca1-4(Fuca1-2Galb1-3)GlcNAcb1-3)Galb1-4Glc-Sp21 & 5 & 11 & 197 \\
\hline 238 & Neu5Aca2-3Galb1-3(6S)GlcNAc-Sp8 & 5 & 4 & 67 \\
\hline 581 & $\begin{array}{l}\text { Galb1-4GIcNAcb1-3Galb1-4GlcNAcb1-3Galb1-4GIcNAcb1-3Galb1-4GIcNAcb1-3Galb1-4GlcNAcb1-2Mana1- } \\
\text { 6(Galb1-4GlcNAcb1-3Galb1-4GlcNAcb1-3Galb1-4GlcNAcb1-3Galb1-4GlcNAcb1-3Galb1-4GIcNAcb1-2Mana1- } \\
\text { 3)Manb1-4GIcNAcb1-4(Fuca1-6)GlcNAcb-Sp19 }\end{array}$ & 5 & 12 & 234 \\
\hline 456 & Neu5Aca2-6Galb1-4GlcNAcb1-6(Fuca1-2Galb1-3GlcNAcb1-3)Galb1-4Glc-Sp21 & 5 & 3 & 59 \\
\hline 396 & $\begin{array}{l}\text { Neu5Aca2-3Galb1-3GlcNAcb1-2Mana1-6(Neu5Aca2-3Galb1-3GlcNAcb1-2Mana1-3)Manb1-4GIcNAcb1- } \\
\text { 4GIcNAc-Sp19 }\end{array}$ & 5 & 2 & 31 \\
\hline 153 & Galb1-4(Fuca1-3)GlcNAcb1-3Galb1-4(Fuca1-3)GlcNAcb-Sp0 & 5 & 6 & 106 \\
\hline 556 & Neu5Gca2-8Neu5Gca2-6Galb1-4GlcNAc-Sp0 & 5 & 6 & 108 \\
\hline 252 & Neu5Aca2-3Galb1-4(Fuca1-3)(6S)GlcNAcb-Sp8 & 5 & 5 & 99 \\
\hline 100 & Gala1-2Galb-Sp8 & 5 & 7 & 134 \\
\hline 260 & Neu5Aca2-3Galb1-4GIcNAcb-Sp8 & 5 & 2 & 37 \\
\hline 132 & Galb1-4GIcNAcb1-6GalNAc-Sp14 & 5 & 10 & 195 \\
\hline 551 & $\begin{array}{l}\text { Galb1-3GlcNAcb1-3Galb1-4GlcNAcb1-2Mana1-6(Galb1-3GlcNAcb1-3Galb1-4GlcNAcb1-2Mana1-3)Manb1- } \\
\text { 4GlcNAcb1-4GlcNAc-Sp25 }\end{array}$ & 5 & 6 & 113 \\
\hline 117 & Gala1-3Galb1-4GIc-Sp10 & 5 & 3 & 53 \\
\hline 510 & Galb1-3(6S)GlcNAcb-Sp8 & 5 & 5 & 100 \\
\hline 160 & Galb1-4GIcNAcb1-3GalNAc-Sp14 & 5 & 4 & 80 \\
\hline 48 & $\mathrm{Neu} 5,9 \mathrm{Ac}_{2} \mathrm{a}-\mathrm{Sp} 8$ & 5 & 3 & 56 \\
\hline 128 & Galb1-3(Fuca1-4)GIcNAc-Sp0 & 5 & 4 & 71 \\
\hline 550 & $\begin{array}{l}\text { Galb1-4GlcNAcb1-3Galb1-4GlcNAcb1-3Galb1-4GlcNAcb1-3Galb1-4GlcNAcb1-2Mana1-6(Galb1-4GlcNAcb1- } \\
\text { 3Galb1-4GlcNAcb1-3Galb1-4GlcNAcb1-3Galb1-4GlcNAcb1-2Mana1-3)Manb1-4GlcNAcb1-4GlcNAcb-Sp25 }\end{array}$ & 5 & 4 & 74 \\
\hline 53 & GlcNAcb1-2Mana1-6(GlcNAcb1-2Mana1-3)Manb1-4GlcNAcb1-4GlcNAcb-Sp13 & 5 & 7 & 141 \\
\hline 426 & $\begin{array}{l}\text { Fuca1-2Galb1-3GIcNAcb1-2Mana1-6(Fuca1-2Galb1-3GIcNAcb1-2Mana1-3)Manb1-4GlcNAcb1-4(Fuca1- } \\
\text { 6)GIcNAcb-Sp22 }\end{array}$ & 5 & 7 & 145 \\
\hline 469 & Glca1-6Glca1-6Glca1-6Glcb-Sp10 & 5 & 4 & 76 \\
\hline 139 & Galb1-3GalNAca-Sp8 & 5 & 8 & 163 \\
\hline 608 & $\begin{array}{l}\text { Neu5Aca2-6Galb1-4GlcNAcb1-3Galb1-4GIcNAcb1-2Mana1-6(Neu5Aca2-6Galb1-4GlcNAcb1-3Galb1- } \\
\text { 4GIcNAcb1-2Mana1-3)Manb1-4GIcNAcb1-4GlcNAcb-Sp12 }\end{array}$ & 5 & 11 & 221 \\
\hline
\end{tabular}




\begin{tabular}{|c|c|c|c|c|}
\hline 580 & $\begin{array}{l}\text { GlcNAcb1-3Galb1-4GlcNAcb1-3Galb1-4GlcNAcb1-3Galb1-4GlcNAcb1-3Galb1-4GlcNAcb1-2Mana1- } \\
\text { 6(GlcNAcb1-3Galb1-4GlcNAcb1-3Galb1-4GlcNAcb1-3Galb1-4GlcNAcb1-3Galb1-4GlcNAcb1-2Mana1-3)Manb1- } \\
\text { 4GlcNAcb1-4(Fuca1-6)GlcNAcb-Sp19 }\end{array}$ & 5 & 5 & 107 \\
\hline 445 & $\begin{array}{l}\text { Fuca1-2Galb1-4 GlcNAcb1-2Mana1-6(Fuca1-2Galb1-4GIcNAcb1-2(Fuca1-2Galb1-4GIcNAcb1-4)Mana1- } \\
\text { 3)Manb1-4GIcNAcb1-4GIcNAcb-Sp12 }\end{array}$ & 5 & 2 & 42 \\
\hline 156 & Galb1-4(6S)Glcb-Sp8 & 5 & 7 & 139 \\
\hline 255 & Neu5Aca2-3Galb1-4(Fuca1-3)GlcNAcb-Sp8 & 5 & 5 & 96 \\
\hline 3 & Mana-Sp8 & 5 & 4 & 76 \\
\hline 7 & Fuca-Sp9 & 5 & 2 & 41 \\
\hline 61 & Fuca1-2Galb1-3GalNAca-Sp8 & 5 & 3 & 56 \\
\hline 58 & Fuca1-2Galb1-3GalNAcb1-3Gala-Sp9 & 5 & 14 & 285 \\
\hline 567 & Galb1-3GIcNAcb1-6(Galb1-3)GalNAc-Sp14 & 5 & 4 & 93 \\
\hline 202 & GlcAb-Sp8 & 5 & 4 & 81 \\
\hline 379 & GalNAcb1-4GlcNAcb1-2Mana1-6(GalNAcb1-4GIcNAcb1-2Mana1-3)Manb1-4GlcNAcb1-4GIcNAc-Sp12 & 5 & 6 & 129 \\
\hline 165 & Galb1-4GIcNAcb1-3Galb1-4GIcb-Sp8 & 5 & 5 & 104 \\
\hline 453 & GalNAcb1-4Galb1-4GIcb-Sp0 & 5 & 5 & 105 \\
\hline 168 & Galb1-4GlcNAcb-Sp0 & 5 & 6 & 128 \\
\hline 506 & GalNAca1-3(Fuca1-2)Galb1-3GIcNAcb1-6GalNAca-Sp14 & 5 & 4 & 92 \\
\hline 486 & $\begin{array}{l}\text { Neu5Aca2-3Galb1-3GlcNAcb1-2Mana1-6(GlcNAcb1-4)(Neu5Aca2-3Galb1-3GlcNAcb1-2Mana1-3)Manb1- } \\
\text { 4GIcNAcb1-4GlcNAc-Sp21 }\end{array}$ & 5 & 3 & 70 \\
\hline 300 & Galb1-3Galb1-4GlcNAcb-Sp8 & 5 & 6 & 141 \\
\hline 457 & $\begin{array}{l}\text { GalNAca1-3(Fuca1-2)Galb1-3GlcNAcb1-2Mana1-6(GalNAca1-3(Fuca1-2)Galb1-3GlcNAcb1-2Mana1-3)Manb1- } \\
\text { 4GlcNAcb1-4(Fuca1-6)GlcNAcb-Sp22 }\end{array}$ & 5 & 5 & 113 \\
\hline 286 & Neu5Gca2-6Galb1-4GIcNAcb-Sp0 & 5 & 7 & 146 \\
\hline 297 & (6S)Galb1-4(6S)GlcNAcb-Sp0 & 5 & 4 & 92 \\
\hline 131 & Galb1-4GIcNAcb1-6GalNAca-Sp8 & 5 & 5 & 115 \\
\hline 83 & GalNAca1-3(Fuca1-2)Galb1-4(Fuca1-3)GIcNAcb-Sp0 & 5 & 5 & 113 \\
\hline 278 & Neu5Acb2-6GalNAca-Sp8 & 5 & 10 & 226 \\
\hline 458 & $\begin{array}{l}\text { Galb1-4GIcNAcb1-6(Galb1-4GIcNAcb1-2)Mana1-6(Galb1-4GIcNAcb1-2Mana1-3)Manb1-4GIcNAcb1-4GIcNAcb- } \\
\text { Sp19 }\end{array}$ & 4 & 2 & 40 \\
\hline 527 & Gala1-3Galb1-3GlcNAcb1-2Mana-Sp0 & 4 & 2 & 52 \\
\hline 56 & $\begin{array}{l}\text { Neu5Aca2-6Galb1-4GIcNAcb1-2Mana1-6(Neu5Aca2-6Galb1-4GIcNAcb1-2Man-a1-3)Manb1-4GIcNAcb1- } \\
\text { 4GlcNAcb-Sp21 }\end{array}$ & 4 & 5 & 110 \\
\hline 135 & Neu5Aca2-6(Galb1-3)GalNAca-Sp8 & 4 & 4 & 91 \\
\hline 415 & GalNAca1-3GalNAcb1-3Gala1-4Galb1-4Glcb-Sp0 & 4 & 3 & 79 \\
\hline 549 & $\begin{array}{l}\text { GlcNAcb1-3Galb1-4GlcNAcb1-3Galb1-4GIcNAcb1-3Galb1-4GlcNAcb1-2Mana1-6(GlcNAcb1-3Galb1- } \\
\text { 4GlcNAcb1-3Galb1-4GlcNAcb1-3Galb1-4GlcNAcb1-2Mana1-3)Manb1-4GIcNAcb1-4GlcNAcb-Sp25 }\end{array}$ & 4 & 2 & 45 \\
\hline 398 & Galb1-4GIcNAcb1-2Mana1-6(GlcNAcb1-2Mana1-3)Manb1-4GIcNAcb1-4GIcNAc-Sp12 & 4 & 2 & 54 \\
\hline 279 & Neu5Acb2-6Galb1-4GIcNAcb-Sp8 & 4 & 4 & 93 \\
\hline
\end{tabular}




\begin{tabular}{|c|c|c|c|c|}
\hline 149 & Galb1-3GlcNAcb-Sp0 & 4 & 1 & 27 \\
\hline 399 & Neu5Aca2-3Galb1-3GIcNAcb1-3GalNAca-Sp14 & 4 & 2 & 57 \\
\hline 375 & $\begin{array}{l}\text { Fuca1-4(Fuca1-2Galb1-3)GlcNAcb1-2Mana1-3(Fuca1-4(Fuca1-2Galb1-3)GlcNAcb1-2Mana1-3)Manb1- } \\
\text { 4GlcNAcb1-4GlcNAcb-Sp19 }\end{array}$ & 4 & 2 & 43 \\
\hline 150 & Galb1-3GlcNAcb-Sp8 & 4 & 3 & 66 \\
\hline 22 & $6 S(3 S) G a l b 1-4(6 S) G l c N A c b-S p 0$ & 4 & 6 & 153 \\
\hline 17 & GlcNAcb-Sp8 & 4 & 12 & 279 \\
\hline 99 & GalNAcb1-4GIcNAcb-Sp8 & 4 & 8 & 191 \\
\hline 276 & Neu5Aca2-8Neu5Aca2-3Galb1-4Glcb-Sp0 & 4 & 13 & 324 \\
\hline 493 & Galb1-4(Fuca1-3)GIcNAcb1-6(Neu5Aca2-6(Neu5Aca2-3Galb1-3)GlcNAcb1-3)Galb1-4Glc-Sp21 & 4 & 2 & 57 \\
\hline 598 & GlcNAcb1-3Galb1-4GlcNAcb1-3Galb1-4GlcNAcb1-3GalNAca-Sp14 & 4 & 1 & 32 \\
\hline 154 & Galb1-4(Fuca1-3)GIcNAcb1-3Galb1-4(Fuca1-3)GIcNAcb1-3Galb1-4(Fuca1-3)GIcNAcb-Sp0 & 4 & 10 & 244 \\
\hline 42 & (6S)Galb1-4Glcb-Sp0 & 4 & 3 & 63 \\
\hline 563 & GalNAcb1-4GIcNAcb1-3GalNAcb1-4GIcNAcb-Sp0 & 4 & 6 & 156 \\
\hline 233 & GalNAcb1-4(Neu5Aca2-3)Galb1-4Glcb-Sp0 & 4 & 6 & 159 \\
\hline 101 & Gala1-3(Fuca1-2)Galb1-3GIcNAcb-Sp0 & 4 & 3 & 85 \\
\hline 427 & $\begin{array}{l}\text { Gala1-3(Fuca1-2)Galb1-4GIcNAcb1-2Mana1-6(Gala1-3(Fuca1-2)Galb1-4GIcNAcb1-2Mana1-3)Manb1- } \\
\text { 4GlcNAcb1-4(Fuca1-6)GIcNAcb-Sp22 }\end{array}$ & 4 & 2 & 53 \\
\hline 488 & Galb1-3GIcNAcb1-6GalNAca-Sp14 & 4 & 2 & 45 \\
\hline 272 & Neu5Aca2-6Galb1-4GIcb-Sp0 & 4 & 2 & 51 \\
\hline 601 & Neu5Aca2-6Galb1-4GlcNAcb1-3Galb1-4GIcNAcb1-6(Galb1-3)GalNAca-Sp14 & 4 & 8 & 196 \\
\hline 522 & Gala1-3(Fuca1-2)Galb1-4GlcNAcb1-2Mana-Sp0 & 4 & 3 & 76 \\
\hline 164 & Galb1-4GIcNAcb1-3Galb1-4GIcb-Sp0 & 4 & 4 & 97 \\
\hline 222 & Fuca1-2(6S)Galb1-4(6S)Glcb-Sp0 & 4 & 16 & 419 \\
\hline 198 & Glcb1-4Glcb-Sp8 & 4 & 3 & 71 \\
\hline 480 & Neu5Aca2-6Galb1-4GIcNAcb1-6GalNAca-Sp14 & 4 & 5 & 131 \\
\hline 16 & GlcNAcb-Sp0 & 4 & 1 & 16 \\
\hline 155 & Galb1-4(6S)Glcb-Sp0 & 4 & 2 & 51 \\
\hline 65 & Fuca1-2Galb1-3GlcNAcb1-3Galb1-4Glcb-Sp8 & 4 & 4 & 101 \\
\hline 79 & Fuca1-3GlcNAcb-Sp8 & 4 & 6 & 154 \\
\hline 70 & Fuca1-2Galb1-4(Fuca1-3)GlcNAcb1-3Galb1-4(Fuca1-3)GlcNAcb1-3Galb1-4(Fuca1-3)GlcNAcb-Sp0 & 4 & 4 & 114 \\
\hline 245 & Neu5Aca2-3Galb-Sp8 & 4 & 6 & 172 \\
\hline 187 & GlcNAcb1-6(GlcNAcb1-4)GalNAca-Sp8 & 4 & 2 & 43 \\
\hline 292 & Galb1-4(Fuca1-3)GlcNAcb1-3Galb1-3(Fuca1-4)GlcNAcb-Sp0 & 4 & 6 & 160 \\
\hline 464 & $\begin{array}{l}\text { Neu5Aca2-6Galb1-4GIcNAcb1-4Mana1-6(GIcNAcb1-4)(Neu5Aca2-6Galb1-4GIcNAcb1-4(Neu5Aca2-6Galb1- } \\
\text { 4GIcNAcb1-2)Mana1-3)Manb1-4GIcNAcb1-4GIcNAcb-Sp21 }\end{array}$ & 4 & 5 & 135 \\
\hline 247 & Neu5Aca2-3Galb1-3GlcNAcb1-3Galb1-4GlcNAcb-Sp0 & 4 & 3 & 72 \\
\hline 176 & GlcNAcb1-6(GlcNAcb1-3)GalNAca-Sp8 & 4 & 6 & 175 \\
\hline 455 & $\begin{array}{l}\text { Gala1-3(Fuca1-2)Galb1-3GlcNAcb1-2Mana1-6(Gala1-3(Fuca1-2)Galb1-3GIcNAcb1-2Mana1-3)Manb1- } \\
\text { 4GlcNAcb1-4(Fuca1-6)GIcNAcb-Sp22 }\end{array}$ & 4 & 4 & 106 \\
\hline 304 & Galb1-4GIcNAca1-6Galb1-4GIcNAcb-Sp0 & 4 & 5 & 146 \\
\hline 322 & Neu5Aca2-8Neu5Aca2-8Neu5Acb-Sp8 & 4 & 3 & 79 \\
\hline
\end{tabular}




\begin{tabular}{|c|c|c|c|c|}
\hline 74 & Fuca1-2Galb1-4GIcNAcb1-3Galb1-4GIcNAcb1-3Galb1-4GIcNAcb-Sp0 & 4 & 5 & 146 \\
\hline 66 & Fuca1-2Galb1-3GlcNAcb1-3Galb1-4Glcb-Sp10 & 4 & 6 & 173 \\
\hline 536 & GalNAca1-3(Fuca1-2)Galb1-3GalNAcb1-3Gala1-4Galb1-4Glc-Sp21 & 4 & 2 & 67 \\
\hline 251 & Neu5Aca2-3Galb1-4(6S)GlcNAcb-Sp8 & 4 & 5 & 134 \\
\hline 334 & GalNAcb1-3Gala1-4Galb1-4GIcNAcb1-3Galb1-4GIcb-Sp0 & 4 & 4 & 104 \\
\hline 147 & Galb1-3GIcNAcb1-3Galb1-4GIcNAcb-Sp0 & 4 & 4 & 111 \\
\hline 40 & (4S)Galb1-4GlcNAcb-Sp8 & 4 & 7 & 207 \\
\hline 133 & GlcNAcb1-6(Galb1-3)GalNAca-Sp8 & 4 & 3 & 73 \\
\hline 405 & Gala1-3Galb1-4GIcNAcb1-3GalNAca-Sp14 & 4 & 5 & 135 \\
\hline 344 & GlcNAca1-4Galb1-3GalNAc-Sp14 & 4 & 6 & 159 \\
\hline 301 & Neu5Aca2-6Galb1-4GIcNAcb1-2Mana1-6(Galb1-4GIcNAcb1-2Mana1-3)Manb1-4GIcNAcb1-4GIcNAcb-Sp12 & 4 & 6 & 162 \\
\hline 225 & GalNAcb1-4(Neu5Aca2-8Neu5Aca2-8Neu5Aca2-8Neu5Aca2-3)Galb1-4GIcb-Sp0 & 3 & 5 & 136 \\
\hline 138 & Neu5Aca2-6(Galb1-3)GlcNAcb1-4Galb1-4Glcb-Sp10 & 3 & 3 & 101 \\
\hline 382 & Galb1-3GIcNAcb1-3Galb1-4GIcNAcb1-6(Galb1-3GlcNAcb1-3)Galb1-4GIcb-Sp0 & 3 & 2 & 5 \\
\hline 196 & Glca1-4Glca-Sp8 & 3 & 5 & 157 \\
\hline 530 & GIcNAcb1-2 Mana1-6(GIcNAcb1-4)(GIcNAcb1-2Mana1-3)Manb1-4GIcNAcb1-4(Fuca1-6)GIcNAc-Sp21 & 3 & 4 & 117 \\
\hline 384 & Galb1-4GIcNAcb1-6(Fuca1-4(Fuca1-2Galb1-3)GIcNAcb1-3)Galb1-4GIc-Sp21 & 3 & 3 & 107 \\
\hline 367 & Neu5Aca2-6GlcNAcb1-4GIcNAcb1-4GIcNAc-Sp21 & 3 & 1 & 27 \\
\hline 305 & Galb1-4GIcNAcb1-6Galb1-4GIcNAcb-Sp0 & 3 & 6 & 195 \\
\hline 562 & GalNAcb1-3GlcNAcb-Sp0 & 3 & 4 & 132 \\
\hline 392 & GalNAcb1-4(Neu5Aca2-3)Galb1-4GIcNAcb1-3GalNAca-Sp14 & 3 & 5 & 163 \\
\hline 49 & Neu5,9Ac2a2-6Galb1-4GlcNAcb-Sp8 & 3 & 4 & 139 \\
\hline 348 & Neu5Aca2-6Galb1-4GIcNAcb1-2Mana1-3Manb1-4GIcNAcb1-4GIcNAc-Sp12 & 3 & 6 & 193 \\
\hline 8 & Rhaa-Sp8 & 3 & 3 & 98 \\
\hline 190 & GlcNAcb1-4GlcNAcb1-4GlcNAcb1-4GlcNAcb1-4GlcNAcb1-Sp8 & 3 & 3 & 104 \\
\hline 327 & $\begin{array}{l}\text { Galb1-4(Fuca1-3)GlcNAcb1-2Mana1-6(Galb1-4(Fuca1-3)GlcNAcb1-2Mana1-3)Manb1-4GlcNAcb1-4GIcNAcb- } \\
\text { Sp20 }\end{array}$ & 3 & 6 & 206 \\
\hline 71 & Fuca1-2Galb1-4(Fuca1-3)GIcNAcb-Sp0 & 3 & 1 & 36 \\
\hline 497 & Fuca1-2(6S)Galb1-3GlcNAcb-Sp0 & 3 & 5 & 169 \\
\hline 393 & GalNAca1-3(Fuca1-2)Galb1-3GalNAca1-3(Fuca1-2)Galb1-4GIcNAcb-Sp0 & 3 & 4 & 157 \\
\hline 41 & (6P)Mana-Sp8 & 3 & 5 & 188 \\
\hline 184 & GIcNAcb1-3Galb1-4GIcNAcb1-3Galb1-4GIcNAcb-Sp0 & 3 & 3 & 106 \\
\hline 273 & Neu5Aca2-6Galb1-4Glcb-Sp8 & 3 & 7 & 252 \\
\hline 129 & Galb1-3(Fuca1-4)GIcNAc-Sp8 & 3 & 1 & 51 \\
\hline
\end{tabular}




\begin{tabular}{|c|c|c|c|c|}
\hline 87 & GalNAca1-3(Fuca1-2)Galb1-4Glcb-Sp0 & 3 & 6 & 235 \\
\hline 535 & GlcNAcb1-3Galb1-4GIcNAcb1-6(GlcNAcb1-3)Galb1-4GIcNAc-Sp0 & 3 & 2 & 68 \\
\hline 340 & GlcNAca1-4Galb1-3GlcNAcb-Sp0 & 3 & 1 & 36 \\
\hline 126 & Galb1-3(Fuca1-4)GlcNAcb1-3Galb1-4(Fuca1-3)GlcNAcb-Sp0 & 3 & 8 & 309 \\
\hline 193 & GlcNAcb1-6GalNAca-Sp14 & 3 & 5 & 178 \\
\hline 293 & Galb1-4GIcNAcb1-3Galb1-3GIcNAcb-Sp0 & 3 & 4 & 145 \\
\hline 274 & Neu5Aca2-6Galb-Sp8 & 3 & 7 & 280 \\
\hline 423 & Gala1-3(Fuca1-2)Galb1-3GlcNAcb1-3GalNAc-Sp14 & 3 & 3 & 110 \\
\hline 81 & Fucb1-3GIcNAcb-Sp8 & 3 & 3 & 108 \\
\hline 451 & GalNAca1-3(Fuca1-2)Galb1-4GIcNAcb1-6(GalNAca1-3(Fuca1-2)Galb1-4GlcNAcb1-3)GalNAc-Sp14 & 3 & 4 & 165 \\
\hline 103 & Gala1-3(Fuca1-2)Galb1-4(Fuca1-3)GIcNAcb-Sp0 & 3 & 10 & 378 \\
\hline 395 & $\begin{array}{l}\text { Gala1-3Galb1-3(Fuca1-4)GlcNAcb1-2Mana1-6(Gala1-3Galb1-3(Fuca1-4)GIcNAcb1-2Mana1-3)Manb1- } \\
\text { 4GlcNAcb1-4GIcNAc-Sp19 }\end{array}$ & 3 & 2 & 90 \\
\hline 170 & Galb1-4GlcNAcb-Sp23 & 3 & 4 & 144 \\
\hline 148 & Galb1-3GIcNAcb1-3Galb1-4Glcb-Sp10 & 2 & 5 & 206 \\
\hline 515 & (3S)GalNAcb1-4GlcNAc-Sp8 & 2 & 4 & 155 \\
\hline 134 & GlcNAcb1-6(Galb1-3)GalNAca-Sp14 & 2 & 4 & 151 \\
\hline 159 & Galb1-4GIcNAcb1-3GalNAca-Sp8 & 2 & 3 & 130 \\
\hline 452 & Neu5Aca2-8Neu5Aca2-3Galb1-3GalNAcb1-4(Neu5Aca2-8Neu5Aca2-3)Galb1-4GIcb-Sp0 & 2 & 3 & 134 \\
\hline 377 & Neu5Aca2-6Galb1-4GlcNAcb1-3GalNAc-Sp14 & 2 & 3 & 140 \\
\hline 411 & Galb1-3GalNAcb1-4(Neu5Aca2-8Neu5Aca2-3)Galb1-4Glcb-Sp0 & 2 & 4 & 176 \\
\hline 166 & Galb1-4GIcNAcb1-6(Galb1-3)GalNAca-Sp8 & 2 & 3 & 131 \\
\hline 23 & $6 \mathrm{~S}(3 \mathrm{~S}) \mathrm{Galb1}-4 \mathrm{GlcNAcb}-\mathrm{Sp} 0$ & 2 & 4 & 165 \\
\hline 90 & GalNAca1-3(Fuca1-2)Galb-Sp18 & 2 & 6 & 251 \\
\hline 14 & Manb-Sp8 & 2 & 2 & 106 \\
\hline 429 & Galb1-4GlcNAcb1-6(Fuca1-2Galb1-3GlcNAcb1-3)Galb1-4Glc-Sp21 & 2 & 4 & 171 \\
\hline 181 & GlcNAcb1-3Galb-Sp8 & 2 & 5 & 239 \\
\hline 208 & Mana1-2Mana1-6(Mana1-2Mana1-3)Mana-Sp9 & 2 & 4 & 168 \\
\hline 231 & GalNAcb1-4(Neu5Aca2-3)Galb1-4GIcNAcb-Sp0 & 2 & 1 & 59 \\
\hline 425 & Gala1-3Galb1-3GIcNAcb1-3GalNAc-Sp14 & 2 & 7 & 319 \\
\hline 192 & GlcNAcb1-6GalNAca-Sp8 & 2 & 3 & 153 \\
\hline 291 & Galb1-4(Fuca1-3)(6S)Glcb-Sp0 & 2 & 6 & 286 \\
\hline 335 & GalNAca1-3(Fuca1-2)Galb1-4GIcNAcb1-3Galb1-4GIcNAcb-Sp0 & 2 & 6 & 274 \\
\hline 434 & $\begin{array}{l}\text { GlcNAcb1-6(GlcNAcb1-2)Mana1-6(GlcNAcb1-4)(GlcNAcb1-4(GlcNAcb1-2)Mana1-3)Manb1-4GlcNAcb1- } \\
\text { 4GlcNAc-Sp21 }\end{array}$ & 2 & 5 & 211 \\
\hline 171 & Galb1-4GIcb-Sp0 & 2 & 3 & 117 \\
\hline 206 & KDNa2-3Galb1-4GIcNAcb-Sp0 & 2 & 4 & 196 \\
\hline 229 & Neu5Aca2-8Neu5Aca2-8Neu5Aca-Sp8 & 2 & 3 & 150 \\
\hline
\end{tabular}




\begin{tabular}{|c|c|c|c|c|}
\hline 465 & $\begin{array}{l}\text { Neu5Aca2-6Galb1-4GIcNAcb1-6(Neu5Aca2-6Galb1-4GlcNAcb1-2)Mana1-6(GlcNAcb1-4)(Neu5Aca2-6Galb1- } \\
\text { 4GlcNAcb1-2Mana1-3)Manb1-4GlcNAcb1-4GIcNAcb-Sp21 }\end{array}$ & 2 & 3 & 146 \\
\hline 20 & Galb1-4GIcNAcb1-6(Galb1-4GlcNAcb1-3)GalNAc-Sp14 & 2 & 2 & 91 \\
\hline 281 & Neu5Gca2-3Galb1-3GlcNAcb-Sp0 & 2 & 1 & 72 \\
\hline 161 & Galb1-4GIcNAcb1-3Galb1-4(Fuca1-3)GlcNAcb1-3Galb1-4(Fuca1-3)GlcNAcb-Sp0 & 2 & 6 & 287 \\
\hline 163 & Galb1-4GIcNAcb1-3Galb1-4GIcNAcb-Sp0 & 2 & 4 & 186 \\
\hline 466 & $\begin{array}{l}\text { Neu5Aca2-6Galb1-4GlcNAcb1-6(Neu5Aca2-6Galb1-4GlcNAcb1-2)Mana1-6(GlcNAcb1-4)(Neu5Aca2-6Galb1- } \\
\text { 4GlcNAcb1-4(Neu5Aca2-6Galb1-4GlcNAcb1-2)Mana1-3)Manb1-4GlcNAcb1-4GlcNAcb-Sp21 }\end{array}$ & 2 & 4 & 182 \\
\hline 284 & Neu5Gca2-3Galb1-4GIcb-Sp0 & 2 & 3 & 141 \\
\hline 115 & Gala1-3Galb1-4GlcNAcb-Sp8 & 2 & 4 & 230 \\
\hline 46 & Neu5Aca2-3(6S)Galb1-4GlcNAcb-Sp8 & 2 & 2 & 110 \\
\hline 277 & Galb1-3(Fuca1-4)GlcNAcb1-3Galb1-3(Fuca1-4)GIcNAcb-Sp0 & 2 & 4 & 205 \\
\hline 265 & Neu5Aca2-6GalNAca-Sp8 & 2 & 4 & 235 \\
\hline 112 & Gala1-3GalNAcb-Sp8 & 2 & 8 & 463 \\
\hline 438 & $\begin{array}{l}\text { Galb1-4GIcNAcb1-6(Galb1-4GIcNAcb1-2)Mana1-6(GlcNAcb1-4)(Galb1-4GlcNAcb1-4(Galb1-4GlcNAcb1- } \\
\text { 2)Mana1-3)Manb1-4GlcNAcb1-4GIcNAc-Sp21 }\end{array}$ & 2 & 4 & 219 \\
\hline 158 & Galb1-4GalNAcb1-3(Fuca1-2)Galb1-4GlcNAcb-Sp8 & 2 & 6 & 330 \\
\hline 256 & Neu5Aca2-3Galb1-4(Fuca1-3)GIcNAcb1-3Galb-Sp8 & 2 & 3 & 206 \\
\hline 554 & Neu5Gca2-8Neu5Aca2-3Galb1-4GlcNAc-Sp0 & 2 & 0 & 18 \\
\hline 503 & GalNAcb1-4(Fuca1-3)(6S)GlcNAcb-Sp8 & 2 & 8 & 483 \\
\hline 319 & Galb1-4GIcNAcb1-2Mana1-6(Neu5Aca2-6Galb1-4GIcNAcb1-2Mana1-3)Manb1-4GIcNAcb1-4GIcNAcb-Sp12 & 2 & 2 & 116 \\
\hline 264 & Neu5Aca2-3Galb1-4GIcb-Sp8 & 2 & 4 & 241 \\
\hline 312 & MurNAcb1-4GlcNAcb-Sp10 & 2 & 5 & 286 \\
\hline 89 & GalNAca1-3(Fuca1-2)Galb-Sp8 & 2 & 7 & 433 \\
\hline 186 & GlcNAcb1-4-MDPLys & 1 & 3 & 226 \\
\hline 389 & Fuca1-2Galb1-3GalNAca1-3(Fuca1-2)Galb1-4Glcb-Sp0 & 1 & 3 & 198 \\
\hline 111 & Gala1-3GalNAca-Sp16 & 1 & 8 & 533 \\
\hline 4 & GalNAca-Sp8 & 1 & 3 & 220 \\
\hline 120 & Gala1-4Galb1-4GlcNAcb-Sp0 & 1 & 3 & 219 \\
\hline 204 & GlcAb1-6Galb-Sp8 & 1 & 4 & 309 \\
\hline 191 & GlcNAcb1-4GlcNAcb1-4GlcNAcb-Sp8 & 1 & 2 & 143 \\
\hline 329 & Neu5,9Ac2a2-3Galb1-3GIcNAcb-Sp0 & 1 & 4 & 335 \\
\hline 172 & Galb1-4GIcb-Sp8 & 1 & 2 & 174 \\
\hline 287 & Neu5Gca-Sp8 & 1 & 3 & 196 \\
\hline 282 & Neu5Gca2-3Galb1-4(Fuca1-3)GlcNAcb-Sp0 & 1 & 3 & 225 \\
\hline 104 & Gala1-3(Fuca1-2)Galb1-4(Fuca1-3)GlcNAcb-Sp8 & 1 & 3 & 233 \\
\hline 67 & Fuca1-2Galb1-3GlcNAcb-Sp0 & 1 & 4 & 357 \\
\hline 435 & Galb1-4GIcNAcb1-2Mana1-6(GlcNAcb1-4)(Galb1-4GIcNAcb1-2Mana1-3)Manb1-4GIcNAcb1-4GIcNAc-Sp21 & 1 & 4 & 375 \\
\hline 402 & GaINAca1-3GalNAcb1-3Gala1-4Galb1-4GlcNAcb-Sp0 & 1 & 2 & 175 \\
\hline 314 & Mana1-6(Mana1-3)Mana1-6(Mana1-3)Manb-Sp10 & 1 & 5 & 392 \\
\hline 271 & Neu5Aca2-6Galb1-4GIcNAcb1-3Galb1-4GIcNAcb-Sp0 & 1 & 4 & 394 \\
\hline 431 & GlcNAcb1-2Mana1-6(GIcNAcb1-4)(GlcNAcb1-2Mana1-3)Manb1-4GlcNAcb1-4GlcNAc-Sp21 & 1 & 4 & 325 \\
\hline
\end{tabular}




\begin{tabular}{|c|c|c|c|c|}
\hline 32 & (3S)Galb1-4(Fuca1-3)GlcNAc-Sp0 & 1 & 3 & 251 \\
\hline 269 & Neu5Aca2-6Galb1-4GlcNAcb-Sp8 & 1 & 6 & 551 \\
\hline 97 & GalNAcb1-4(Fuca1-3)GIcNAcb-Sp0 & 1 & 5 & 442 \\
\hline 30 & (3S)Galb1-3GlcNAcb-Sp0 & 1 & 4 & 401 \\
\hline 175 & GlcNAcb1-2Galb1-3GalNAca-Sp8 & 1 & 5 & 536 \\
\hline 113 & Gala1-3Galb1-4(Fuca1-3)GlcNAcb-Sp8 & 1 & 2 & 268 \\
\hline 125 & Galb1-2Galb-Sp8 & 1 & 4 & 439 \\
\hline 394 & Gala1-3Galb1-3GIcNAcb1-2Mana1-6(Gala1-3Galb1-3GIcNAcb1-2Mana1-3)Manb1-4GIcNAcb1-4GIcNAc-Sp19 & 1 & 12 & 1343 \\
\hline 288 & Neu5Aca2-3Galb1-4GIcNAcb1-6(Galb1-3)GalNAca-Sp14 & 1 & 2 & 276 \\
\hline 313 & Mana1-6Manb-Sp10 & 1 & 7 & 780 \\
\hline 537 & Gala1-3(Fuca1-2)Galb1-3GalNAcb1-3Gala1-4Galb1-4Glc-Sp21 & 1 & 3 & 433 \\
\hline 442 & GalNAcb1-6GalNAcb-Sp8 & 1 & 6 & 804 \\
\hline 600 & Neu5Aca2-3Galb1-4GlcNAcb1-3Galb1-4GlcNAcb1-6(Galb1-3)GalNAca-Sp14 & 1 & 2 & 352 \\
\hline 504 & (3S)GalNAcb1-4(Fuca1-3)GlcNAcb-Sp8 & 1 & 4 & 595 \\
\hline 294 & Neu5Aca2-3Galb1-3GlcNAcb1-3Galb1-3GlcNAcb-Sp0 & 1 & 4 & 624 \\
\hline 199 & Glcb1-6Glcb-Sp8 & 1 & 4 & 756 \\
\hline 414 & GalNAca1-3(Fuca1-2)Galb1-4GlcNAcb1-3GalNAca-Sp14 & 1 & 3 & 548 \\
\hline 262 & Fuca1-2Galb1-4(6S)GIcb-Sp0 & 1 & 4 & 767 \\
\hline 137 & Neu5Acb2-6(Galb1-3)GalNAca-Sp8 & 1 & 4 & 847 \\
\hline 526 & Neu5Aca2-3Galb1-3GlcNAcb1-2Mana-Sp0 & 1 & 11 & 2164 \\
\hline 127 & Galb1-3GIcNAcb1-3Galb1-4(Fuca1-3)GIcNAcb-Sp0 & 0 & 1 & 204 \\
\hline 239 & Neu5Aca2-3Galb1-3(Fuca1-4)GlcNAcb-Sp8 & 0 & 2 & 471 \\
\hline 538 & Galb1-3GalNAcb1-3Gal-Sp21 & 0 & 1 & 501 \\
\hline 11 & Neu5Acb-Sp8 & 0 & 3 & 985 \\
\hline 544 & $\begin{array}{l}\text { Fuca1-2Galb1-4GIcNAcb1-3Galb1-4GIcNAcb1-2Mana1-6(Fuca1-2Galb1-4GIcNAcb1-3Galb1-4GIcNAcb1- } \\
\text { 2Mana1-3)Manb1-4GIcNAcb1-4GIcNAcb-Sp24 }\end{array}$ & 0 & 7 & 3673 \\
\hline 533 & Fuca1-4(Galb1-3)GIcNAcb1-2 Mana-Sp0 & 0 & 4 & 3077 \\
\hline 496 & Galb1-4(Fuca1-3)GlcNAcb1-2Mana-Sp0 & 0 & 2 & 1632 \\
\hline 525 & Gala1-3(Fuca1-2)Galb1-3GIcNAcb1-6GalNAc-Sp14 & 0 & 4 & 5615 \\
\hline 390 & Fuca1-2Galb1-3GalNAca1-3(Fuca1-2)Galb1-4GIcNAcb-Sp0 & 0 & 4 & 13790 \\
\hline 44 & (6S)Galb1-4GIcNAcb-Sp8 & 0 & 1 & 5959 \\
\hline
\end{tabular}




\begin{tabular}{|c|c|c|c|c|}
\hline 499 & Fuca1-2Galb1-4GIcNAcb1-2Mana-Sp0 & 0 & 11 & 75195 \\
\hline 296 & $4 \mathrm{~S}(3 \mathrm{~S}) \mathrm{Galb1} 1-4 \mathrm{GlcNAcb}-\mathrm{Sp} 0$ & 0 & 5 & -50454 \\
\hline 19 & Galb1-4GIcNAcb1-6(Galb1-4GIcNAcb1-3)GalNAca-Sp8 & 0 & 1 & -1826 \\
\hline 174 & GIcNAca1-6Galb1-4GIcNAcb-Sp8 & 0 & 5 & -6566 \\
\hline 599 & Galb1-4GIcNAcb1-3Galb1-3GalNAca-Sp14 & 0 & 4 & -3042 \\
\hline 144 & Galb1-3GalNAcb1-4(Neu5Aca2-3)Galb1-4GIcb-Sp0 & 0 & 4 & -2714 \\
\hline 77 & Fuca1-2Galb1-4GIcb-Sp0 & 0 & 7 & -3304 \\
\hline 463 & $\begin{array}{l}\text { Neu5Aca2-6Galb1-4GIcNAcb1-2Mana1-6(GIcNAcb1-4)(Neu5Aca2-6Galb1-4GlcNAcb1-2Mana1-3)Manb1- } \\
\text { 4GlcNAcb1-4GIcNAcb-Sp21 }\end{array}$ & 0 & 2 & -890 \\
\hline 302 & Galb1-4GlcNAcb1-6(Galb1-4GlcNAcb1-3)Galb1-4GlcNAc-Sp0 & 0 & 4 & -1711 \\
\hline 440 & Galb1-6Galb-Sp10 & 0 & 4 & -1351 \\
\hline 437 & $\begin{array}{l}\text { Galb1-4GlcNAcb1-6(Galb1-4GlcNAcb1-2)Mana1-6(GlcNAcb1-4)(Galb1-4GlcNAcb1-2Mana1-3)Manb1- } \\
\text { 4GlcNAcb1-4GlcNAc-Sp21 }\end{array}$ & 0 & 4 & -1343 \\
\hline 180 & GlcNAcb1-3GalNAca-Sp14 & 0 & 3 & -1043 \\
\hline 413 & Gala1-3(Fuca1-2)Galb1-4GIcNAcb1-3GalNAca-Sp14 & 0 & 3 & -685 \\
\hline 266 & Neu5Aca2-6GalNAcb1-4GlcNAcb-Sp0 & 0 & 2 & -624 \\
\hline 136 & Neu5Aca2-6(Galb1-3)GalNAca-Sp14 & 0 & 8 & -1871 \\
\hline 289 & Galb1-3GIcNAcb1-3Galb1-3GIcNAcb-Sp0 & -1 & 3 & -559 \\
\hline 177 & GlcNAcb1-6(GlcNAcb1-3)GalNAca-Sp14 & -1 & 5 & -968 \\
\hline 422 & Fuca1-2Galb1-3GIcNAcb1-3GalNAc-Sp14 & -1 & 3 & -485 \\
\hline 592 & Neu5Aca2-3Galb1-4GIcNAcb1-3Galb1-4GIcNAcb1-3GalNAca-Sp14 & -1 & 1 & -121 \\
\hline 479 & Neu5Aca2-3Galb1-4GIcNAcb1-6GalNAca-Sp14 & -1 & 1 & -172 \\
\hline 82 & GalNAca1-3(Fuca1-2)Galb1-3GIcNAcb-Sp0 & -1 & 2 & -326 \\
\hline 433 & GlcNAcb1-6(GlcNAcb1-2)Mana1-6(GIcNAcb1-4)(GlcNAcb1-2Mana1-3)Manb1-4GIcNAcb1-4GIcNAc-Sp21 & -1 & 3 & -364 \\
\hline 212 & Mana1-2Mana1-6(Mana1-2Mana1-3)Mana1-6(Mana1-2Mana1-2Mana1-3)Manb1-4GIcNAcb1-4GIcNAcb-Sp12 & -1 & 0 & -43 \\
\hline 517 & Galb1-4(6P)GlcNAcb-Sp0 & -1 & 4 & -361 \\
\hline 338 & GlcNAca1-4Galb1-4GlcNAcb1-3Galb1-4GIcNAcb1-3Galb1-4GlcNAcb-Sp0 & -1 & 3 & -268 \\
\hline
\end{tabular}




\begin{tabular}{|c|c|c|c|c|}
\hline 603 & $\begin{array}{l}\text { Neu5Aca2-3Galb1-4GIcNAcb1-3Galb1-4GIcNAcb1-2Mana1-6(Neu5Aca2-3Galb1-4GlcNAcb1-3Galb1- } \\
\text { 4GIcNAcb1-2Mana1-3)Manb1-4GlcNAcb1-4GIcNAcb-Sp12 }\end{array}$ & -1 & 9 & -722 \\
\hline 85 & GalNAca1-3(Fuca1-2)Galb1-4GlcNAcb-Sp0 & -1 & 4 & -253 \\
\hline 505 & Fuca1-2Galb1-3GIcNAcb1-6(Fuca1-2Galb1-3GIcNAcb1-3)GalNAca-Sp14 & -2 & 4 & -235 \\
\hline 218 & Neu5Aca2-3Galb1-4GlcNAcb1-3Galb1-4(Fuca1-3)GlcNAcb-Sp0 & -2 & 3 & -189 \\
\hline 189 & GlcNAcb1-4GlcNAcb1-4GIcNAcb1-4GIcNAcb1-4GlcNAcb1-4GIcNAcb1-Sp8 & -2 & 5 & -333 \\
\hline 92 & GalNAca1-3Galb-Sp8 & -2 & 3 & -157 \\
\hline 528 & GalNAcb1-4GlcNAcb1-2Mana-Sp0 & -2 & 5 & -255 \\
\hline 45 & (6S)Galb1-4(6S)Glcb-Sp8 & -2 & 2 & -91 \\
\hline 303 & GlcNAcb1-6(Galb1-4GlcNAcb1-3)Galb1-4GlcNAc-Sp0 & -2 & 3 & -162 \\
\hline 523 & GalNAca1-3(Fuca1-2)Galb1-4 GIcNAcb1-2Mana-Sp0 & -2 & 3 & -145 \\
\hline 93 & GalNAca1-4(Fuca1-2)Galb1-4GlcNAcb-Sp8 & -2 & 7 & -299 \\
\hline 310 & GlcNAcb1-4GlcNAcb-Sp10 & -3 & 6 & -186 \\
\hline 230 & Neu5Aca2-3(6S)Galb1-4(Fuca1-3)GIcNAcb-Sp8 & -3 & 4 & -124 \\
\hline 548 & $\begin{array}{l}\text { Galb1-4GlcNAcb1-3Galb1-4GlcNAcb1-3Galb1-4GlcNAcb1-2Mana1-6(Galb1-4GlcNAcb1-3Galb1-4GlcNAcb1- } \\
\text { 3Galb1-4GlcNAcb1-2Mana1-3)Manb1-4GlcNAcb1-4GlcNAcb-Sp24 }\end{array}$ & -3 & 7 & -223 \\
\hline 157 & Galb1-4GalNAca1-3(Fuca1-2)Galb1-4GIcNAcb-Sp8 & -4 & 10 & -259 \\
\hline
\end{tabular}

